
Sonja Sälzle | Linda Vogt | Jennifer Blank

André Bleicher | Ingrid Scholz | Nadja Karossa Renate Stratmann | Thomas D'Souza

Entwicklungspfade für Hochschule und Lehre nach der Corona-Pandemie 
Sonja Sälzle | Linda Vogt | Jennifer Blank | André Bleicher Ingrid Scholz | Nadja Karossa | Renate Stratmann | Thomas D'Souza

\section{Entwicklungspfade für Hochschule und Lehre nach der Corona-Pandemie}

Eine qualitative Studie mit Hochschulleitungen, Lehrenden und Studierenden

Tectum Verlag 
@ Sonja Sälzle | Linda Vogt | Jennifer Blank | André Bleicher | Ingrid Scholz

Nadja Karossa | Renate Stratmann | Thomas D'Souza

Eine Studie des Instituts für Bildungstransfer der Hochschule Biberach in Zusammenarbeit mit der Geschäftsstelle der Studienkommission für Hochschuldidaktik an Hochschulen für Angewandte Wissenschaften in Baden-Württemberg

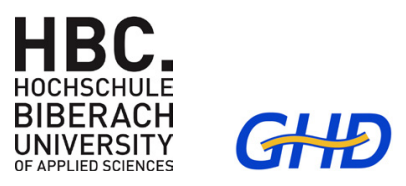

unter Beteiligung der Hochschule Aalen I Hochschule Albstadt-Sigmaringen I Hochschule Biberach Hochschule Esslingen I Evangelischen Hochschule Freiburg I Hochschule Heilbronn I Hochschule Karlsruhe - Technik und Wirtschaft I Hochschule der Medien I Hochschule für Wirtschaft und Umwelt NürtingenGeislingen I Hochschule Reutlingen I Hochschule für Gestaltung Schwäbisch Gmünd

Die Deutsche Nationalbibliothek verzeichnet diese Publikation in der Deutschen Nationalbibliografie; detaillierte bibliografische Angaben sind im Internet über http://dnb.d-nb.de abrufbar.

1. Auflage 2021

Publiziert von

Tectum - ein Verlag in der

Nomos Verlagsgesellschaft $\mathrm{mbH} \&$ Co. KG

Waldseestraße 3-5 | 76530 Baden-Baden

www.tectum-verlag.de

Gesamtherstellung:

Tectum - ein Verlag in der

Nomos Verlagsgesellschaft mbH \& Co. KG

Waldseestraße 3-5 | 76530 Baden-Baden

Umschlaggestaltung: Tectum Verlag, unter Verwendung des Bildes

\# 442224310 von BT Image | www.shutterstock.de

ISBN (Print) 978-3-8288-4659-3

ePDF $978-3-8288-7735-1$

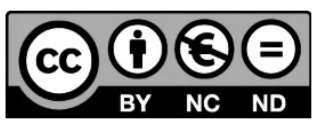

Dieses Werk ist lizenziert unter einer

Creative Commons Namensnennung - Nicht kommerziell Keine Bearbeitungen 4.o International Lizenz. 


\section{Inhaltsverzeichnis}

Abbildungsverzeichnis ................................................. IX

Abkürzungsverzeichnis .................................................. XI

Geleitwort .................................................................... XII

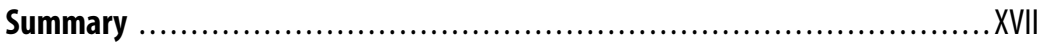

1 Ausgangssituation: digitale Lehre an Hochschulen ...................... 1

2 Aktueller Stand: Studien zum Thema Lehre und Corona-Pandemie ........ 11

2.1 Quantitativer Forschungshintergrund ................................ 12

2.1.1 Hochschulinterne Studien ................................... 12

2.1.2 Hochschulübergreifende und nationale Studien .................... 15

2.1.3 Internationale Studien ........................................... 20

2.2 Qualitativer Forschungshintergrund .................................. 23

2.2.1 Hochschulübergreifende und nationale Studien .................... 23

2.2.2 Internationale Studien ..................................... 26

3 Forschungsdesign: Forschungsfragen und Methode .................... 29

3.1 Forschungsfragen und Aufbau der Studie .............................. 30

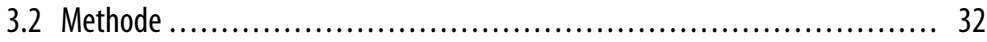

3.2.1 Qualitative Inhaltsanalyse .................................. 32

3.2.2 Sampling ............................................... 33

4 Zugang 1: Kontingenzbedingungen und Zukunftsperspektiven aus Sicht der Hochschulleitungen .............................................. 37

4.1 Untersuchungsgegenstand und methodische Vorgehensweise ............... 37

4.2 Rahmenbedingungen ........................................... 39 
4.3 Umsetzung ..................................................... 43

4.4 Herausforderungen ............................................... 49

4.5 Chancen .................................................................. 55

4.6 Anforderungen .................................................. 61

5 Zugang 2: Die Corona-Semester aus Sicht der Lehrenden und

Studierenden ........................................................... 67

5.1 Untersuchungsgegenstand und methodische Vorgehensweise ..............6 68

5.2 Konkrete Lehr-/Lernsituation ..................................... 70

5.3 Gesamtsituation ................................................ 82

5.4 Blick in die Zukunft .............................................. 92

5.4.1 Didaktische und technische Ebene ........................... 92

5.4.2 Kommunikation und Interaktion ................................. 102

5.4.3 Rahmenbedingungen/Strukturen ................................ 107

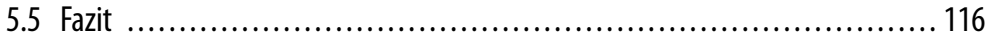

6 Zugang 3: Good Practices und Potenziale digitaler Lehre .................. 119

6.1 Untersuchungsgegenstand und methodische Vorgehensweise .............. 120

6.2 Good Practices: Die Lehrenden als Treiber guter Hochschullehre ............... 122

6.2.1 Good Practice: Inverted Classroom mit Videos ......................... 124

6.2.2 Good Practice: Virtuelle Projektarbeit .............................. 127

6.2.3 Good Practice: Interaktion in der Onlineveranstaltung ............... 129

6.2.4 Good Practice: Digitale Prüfungsformate ......................... 132

6.3 Die Zukunft digitaler Lehre aus der Sicht der treibenden Lehrenden ........... 134

6.3.1 Chancen digitaler Lehre ......................................... 135

6.3.2 Wünsche für die digitale Lehre in Zukunft .......................... 137

7 Leitmotive und Handlungsimpulse für eine Pfadentwicklung nach der Corona-Pandemie ..................................................... 143

7.1 Theoretische Einbettung und Kontextualisierung ........................ 143 
7.2 Leitmotive in der Dimension Akteur*innen .............................. 149

7.2.1 Belastung und Bedürfnisse ....................................... 150

7.2.2 Begegnungen und Kommunikation .............................. 152

7.2.3 Selbstverständnis von Lehren und Lernen ........................... 154

7.3 Handlungsimpulse in der Dimension Akteur*innen ....................... 155

7.3.1 Arbeits- und Lernwelten neu denken ................................ 156

7.3.2 Studierendenzentriert denken ................................ 160

7.4 Leitmotive in der Dimension Strukturation .............................. 165

7.4.1 Handeln unter Unsicherheit ..................................... 167

7.4.2 Kompetenzgap und Kompetenzaufbau .............................. 170

7.4.3 Vorteile der Präsenz und des Digitalen ........................... 171

7.5 Handlungsimpulse in der Dimension Strukturation ........................ 174

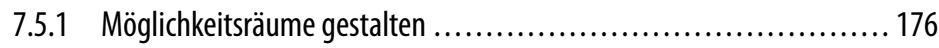

7.5.2 Digitale Elemente im Rahmen von Präsenzhochschulen denken ........ 179

7.6 Leitmotive in der Dimension Strategie ................................... 183

7.6.1 Innovation und Potenzial ....................................... 184

7.6.2 Digitalisierung und Wettbewerb ............................... 186

7.7 Handlungsimpulse in der Dimension Strategie ........................... 188

7.7.1 Eigenraum strategisch gestalten ................................ 190

7.7.2 Hochschulumwelt gemeinsam gestalten .......................... 195

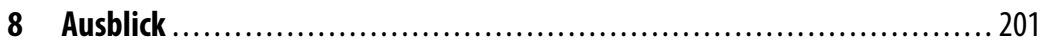

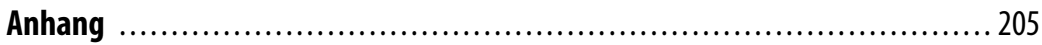

Anhang 1: Interviewleitfaden Hochschulleitungen ............................ 205

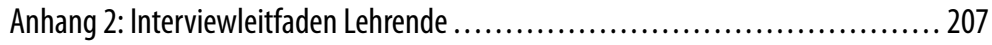

Anhang 3: Interviewleitfaden Studierende .................................... 209

Anhang 4: Interviewleitfaden treibende Lehrende .......................... 211

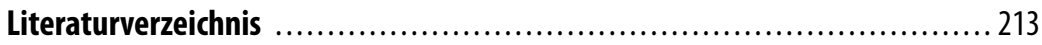




\section{Abbildungsverzeichnis}

Abbildung 1: Ergebnisse der Studie XIX

Abbildung 2: Möglichkeitsraum 9

Abbildung 3: Forschungsfragen der drei Zugänge 31

Abbildung 4: Größe der beteiligten HAWs (2021) 34

Abbildung 5: Sampling in den drei Forschungszugängen $\quad 35$

Abbildung 6: Teilforschungsfragen Zugang 1 im Gesamtzusammenhang $\quad 38$

$\begin{array}{lll}\text { Abbildung 7: } & \text { Leitfadenstruktur Zugang } 1 & 38\end{array}$

Abbildung 8: Zentrale Aspekte der Rahmenbedingungen $\quad 40$

Abbildung 9: Zentrale Aspekte der Umsetzung 43

Abbildung 10: Zentrale Aspekte der Herausforderungen 50

Abbildung 11: Zentrale Aspekte der Chancen 56

Abbildung 12: Zentrale Aspekte der Anforderungen 61

Abbildung 13: Aufbau Forschungszugang 2 67

Abbildung 14: Teilforschungsfragen Zugang 2 im Gesamtzusammenhang $\quad 68$

Abbildung 15: Aspekte für den Bereich Lehr-/Lernsituation 81

Abbildung 16: Aspekte Hometeaching- und Homestudying-

Situation 91

Abbildung 17: Blick in die Zukunft - didaktische und

technische Aspekte 101

Abbildung 18: Blick in die Zukunft - Aspekte der

Kommunikation und der Interaktion 
Abbildung 19: Blick in die Zukunft - Aspekte der

Rahmenbedingungen und der Strukturen

Abbildung 20: Gestaltungsfelder innerhalb des

Möglichkeitsraums

Abbildung 21: Teilforschungsfragen Zugang 3 im Gesamtzusammenhang

Abbildung 22: Leitfragenstruktur Zugang 3

Abbildung 23: Übersicht der Good Practices

Abbildung 24: Übersicht der Chancen digitaler Lehre

Abbildung 25: Wünsche zur digitalen Lehre

Abbildung 26: Übersicht Kontingenzbedingungen

Abbildung 27: Zusammenhang zwischen Handlung, Struktur und Strategie

Abbildung 28: Übersicht über die Leitmotive und die Handlungsfelder

Abbildung 29: Leitmotive in der Dimension Akteur*innen

Abbildung 30: Handlungsimpulse in der Dimension Akteur*innen

Abbildung 31: Leitmotive in der Dimension Strukturation

Abbildung 32: Handlungsimpulse im Bereich Strukturation

Abbildung 33: Leitmotive in der Dimension Strategie 


\section{Abkürzungsverzeichnis}

AR

C19 ISWS

FGL

FGS

HAW

HS

HSL

IL

$\mathrm{KI}$

LHG

MOOC

MWK

SoSe

SPO

VR

WiSe
Augmented Reality

International COVID-19-Student Well-being Survey

Fokusgruppen-Lehrende (Interviewpartner*innen Zugang 2)

Fokusgruppen-Studierende (Interviewpartner`innen Zugang 2)

Hochschule für Angewandte Wissenschaften

Hochschule

Hochschulleitungen (Interviewpartner*innen Zugang 1)

Innovative ${ }^{\star} r$ Lehrende ${ }^{\star} r$ (Interviewpartner ${ }^{\star}$ innen Zugang 3)

Künstliche Intelligenz

Landeshochschulgesetz

Massive Open Online Course

Ministerium für Wissenschaft, Forschung und Kunst

Baden-Württemberg

Sommersemester

Studien- und Prüfungsordnung

Virtual Reality

Wintersemester 


\section{Geleitwort}

Die Corona-Pandemie hat der Digitalisierung in der Hochschullehre, wie auch in vielen anderen Bereichen der Gesellschaft, einen immensen Schub verliehen. Politik und Wirtschaft fordern schon seit Längerem, die digitale Transformation an Hochschulen zu beschleunigen. Digitalisierung in der Lehre ist also nicht erst seit den Auswirkungen dieser Krise ein viel diskutiertes Thema. Doch anstatt Digitalisierung durch Förderung von Einzelinitiativen und Leuchtturmprojekten in Studium und Lehre nur zu beforschen und punktuell zu unterstützen, führt die aktuelle Situation dazu, dass derzeit an Hochschulen umfassend und flächendeckend (fast) ausschließlich digital unterrichtet wird. Wenn auch unfreiwillig, so wurden die Hochschulen durch die Corona-Pandemie doch unversehens zu einer Art Reallabor der digitalen Lehre - und dies seit nunmehr drei Semestern. Alle, sowohl die der Digitalisierung gegenüber skeptischen als auch die ihr gegenüber aufgeschlossen eingestellten Lehrenden, mussten ihre kompletten Lehrveranstaltungen in den digitalen, virtuellen Raum verlegen. Und auch die Studierenden mussten im Homestudying ihre gewohnten Lernumgebungen an den Hochschulen mit ihren Privaträumen tauschen. Obwohl die Bewältigung dieser Krise und die damit einhergehende Ad-Hoc-Umstellung der Lehre auf rein digitale Lehr-/Lernsettings von vielen Beteiligten - Hochschulleitungen, Lehrenden und Studierenden - im Grundsatz als positiv bewertet wurden, stellt sich die Frage nach den Auswirkungen dieser Erfahrungen auf die Zukunftsperspektiven von Hochschulen: Wie sehen Hochschulen und Hochschullehre nach der Pandemie aus?

Genau dieser Frage geht die vorliegende Studie nach. Sie nutzt die Chance, aus den Erfahrungen der letzten digitalen Semester Schlussfolgerungen zu den Gelingensbedingungen des digitalen Miteinanders an Hochschulen als Arbeits-, Lehr- und Lernwelten zu ziehen und lässt sowohl Hochschulleitungen als auch Lehrende und Studierende zu Wort kommen. In Ergänzung zu vielen bisher vorliegenden Studien, in denen die Lehre unter Corona-Bedingungen quantitativ untersucht wurde, wird hier ein qualitativer Forschungsansatz gewählt. Dieser erlaubt es, in 
Tiefe und Detail ein umfassendes Verständnis von Hochschullehre in der digital bestimmten Sondersituation zu entwickeln. Dies ist erforderlich, weil Hochschulen - auch schon jenseits aller Digitalisierung - hochkomplexe Systeme sind.

Diese Komplexität bildet sich sowohl in der Institution Hochschule als auch in der konkreten Lehr-/Lernsituation ab. Durch die Corona-Pandemie sind dabei nicht nur Prozesse und Methoden neu definiert worden, sondern es sind vor allem auch die zwischenmenschliche Beziehung sowie die Hochschule als Arbeits-, Lehr- und Lernwelt in den Fokus getreten. Prozesse des Lehrens und Lernens leben von der Interaktion und dem persönlichen Kontakt von Lehrenden und Studierenden miteinander, aber auch untereinander. Kleinere Studierendengruppen und eine eher vertrauensvoll verbindliche Atmosphäre erleichtern einen solchen Kontakt und sind wichtige Charakteristika der Hochschulen für Angewandte Wissenschaften (HAW). Erfahrungen in diesem Hochschultyp sind der Gegenstand dieser Studie: Elf der 24 Hochschulen für Angewandte Wissenschaften in Baden-Württemberg beteiligten sich daran. Die in der aktuellen Situation stark eingeschränkten Begegnungsmöglichkeiten in Präsenz und die damit einhergehenden veränderten Kommunikations- und Kooperationsformate stellten die Hochschulen vor neue Herausforderungen. Ähnliches gilt auch für die an den HAWs typische Praxis- und Anwendungsorientierung der Lehre, die häufig vor allem durch studentische Projekte und Laborveranstaltungen erreicht wird. Lehr- und Lernformate aber auch das hochschulische Miteinander neben den Lehrveranstaltungen, bzw. die Organisations- und Entscheidungsprozesse an Hochschulen sind dabei nicht eins zu eins vom Analogen ins Digitale zu übertragen und erfordern neue Konzepte für die digitale Welt. Wie diese digitale Welt nach der Corona-Pandemie aussehen soll, liegt in den gestaltenden Händen von allen beteiligen Akteur*innen gemeinsam.

Die Herausforderung in der Gestaltung zukünftiger Entwicklungspfade für Hochschule und Lehre nach der Corona-Pandemie liegt dann vor allem auch darin, dass sich stets neue Optionen für zukünftige Strategien entwickeln. Dies bedeutet, dass wir den Möglichkeitsraum der digitalen Lehre und der Zukunft der Hochschule im Allgemeinen immer wieder neu ausloten und Entwicklungspotentiale erkennen müssen. Eine rein digitale Hochschule ist sicher auf Dauer keine Option. Die Hochschul- 
lehre wird in Zukunft mit hoher Wahrscheinlichkeit aus einer Kombination von digitalen Elementen und klassischer Präsenzlehre bestehen. Wie kann ein solches hybrides oder kombiniertes Szenario aussehen? Welche Bedingungen und welche Faktoren werden im Lichte der in der Krise gemachten Erfahrungen ein lernförderliches Studium mit digitalen Bestandteilen kennzeichnen? Die vorliegende Studie wird diese Frage beleuchten und mögliche Entwicklungspfade für eine Hochschullehre nach der Corona-Pandemie aufzeigen.

Eine solche Studie gelingt nicht ohne die Unterstützung verschiedenster Akteur*innen und Einrichtungen. Die folgenden Hochschulen stellten Probanden für die Befragungen zur Verfügung: Hochschule Aalen, Hochschule Albstadt-Sigmaringen, Hochschule Esslingen, Evangelische Hochschule Freiburg, Hochschule Heilbronn, Hochschule Karlsruhe Technik und Wirtschaft, Hochschule der Medien, Hochschule für Wirtschaft und Umwelt Nürtingen-Geislingen, Hochschule Reutlingen und Hochschule für Gestaltung Schwäbisch Gmünd. Den Kolleg*innen gebührt großer Dank. Daran schließt sich der Dank an die Interviewpartner*innen an - Hochschulleitungen, Lehrenden und Studierenden -, die durch ihre differenzierte Schilderung der Corona-Semester die wichtigste Grundlage der Studie schufen. Gerade in der jetzigen Situation ist es nicht selbstverständlich, zusätzliche Zeit für die Teilnahme an einer solchen Untersuchung aufzubringen. Die Geschäftsstelle der Studienkommission für Hochschuldidaktik an Hochschulen für Angewandte Wissenschaften in Baden-Württemberg (GHD) unterstützte die Studie. Wir bedanken uns insbesondere bei dem Leiter der GHD, Prof. Dipl.Phys. Hans-Peter Voss. Die Impulse der GHD waren aus didaktischer Perspektive sehr wertvoll.

Schließlich haben wir Prof. Dr. Dr. h.c. Bastian Kaiser, Hochschule für Forstwirtschaft Rottenburg, zu danken, der in seiner Eigenschaft als Vorsitzender der Rektorenkonferenz der HAWs in Baden-Württemberg an entscheidender Stelle einen wichtigen Beitrag dazu leistete, die Rahmenbedingungen für die Erstellung der Studie zu schaffen.

Biberach und Karlsruhe im Mai 2021

Die Autorinnen und Autoren 


\section{Summary}

\section{Ausgangslage}

Durch die Corona-Pandemie wurden Hochschulen zum Sommersemester 2020 in eine völlig neue Situation geworfen und mussten sich ad hoc auf die Umstellung des Hochschulbetriebs auf ein digitales Format einstellen. An diesem Punkt gab es keine Möglichkeit zur strategischen Planung, vielmehr wurde im Sinne eines Muddling-Through-Prozesses die Aufgabe der Aufrechterhaltung des Hochschulbetriebs bestritten. Nach einem Jahr unter Corona-Bedingungen stellt sich vermehrt die Frage nach den Entwicklungsmöglichkeiten. Aus dem Übergangszustand, den es zu bewältigen galt, ist ein Möglichkeitsraum geworden, der definiert und gestaltet werden kann und muss. Hochschulen stehen vor der enormen Herausforderung, die Lessons Learned der Corona-Pandemie in eine strategische Pfadentwicklung zu überführen.

\section{Forschungsfrage}

Im Fokus der Studie steht die Forschungsfrage: Welche Entwicklungspfade für Hochschule und Lehre post Corona können aus den Erfahrungen der digitalen Semester abgeleitet werden? Um diese Frage zu bearbeiten, wurden in drei Zugängen verschiedene Gruppen von Akteur*innen (Hochschulleitungen, Lehrende, Studierende) befragt. Ziel war, auf einer empirischen Datenbasis ein ganzheitliches und differenziertes Bild der beiden Corona-Semester an den Hochschulen für Angewandte Wissenschaften (HAW) in Baden-Württemberg zu erfassen und davon ausgehend handlungsleitende Impulse für eine Pfadentwicklung pst Corona abzuleiten.

\section{Methodik}

Die empirische Datenerhebung basierte auf leitfadengestützten Einzelinterviews und Fokusgruppen im Zeitraum von Januar bis März 2021. Die Auswertung erfolgte auf der Grundlage der inhaltlich-strukturierenden Inhaltsanalyse von Kuckartz (2018). Das qualitative Forschungs- 
design zielte darauf ab, in den drei Zugängen themenfokussiert auf das subjektive Erfahrungswissen und die unterschiedlichen Perspektiven der verschiedenen Zielgruppen zurückzugreifen, um differenzierte Bedeutungs- und Begründungszusammenhänge zu erfassen und darauf basierend ein möglichst umfassendes Bild der Corona-Semester zu rekonstruieren. An der Studie nahmen elf von insgesamt 24 HAWs in Baden-Württemberg teil.

\section{Ergebnisse}

Die Interviews gaben Aufschluss über die Ausgangslage vor Corona, die Herausforderungen und Chancen durch die Pandemie und die notwendigen Anforderungen nach der Corona-Pandemie. Ausgehend von der Darstellung der Gestaltung von Hochschule durch Hochschulleitungen, Lehrende und Studierende wurden aus den Daten zentrale Leitmotive erfasst, die den Dimensionen Akteur*innen, Strukturation (die Wechselseitigkeit von Struktur und Handlung) und Strategie zugeordnet wurden (s. Abbildung 1). Auf den acht Leitmotiven basierend wurden aus jeder Dimension Handlungsfelder abgeleitet.

\section{Dimension Akteur*innen: Arbeits- und Lernwelten neu denken}

- Es ist zu definieren, wie das digitale Miteinander gestaltet wird.

- Alle Beteiligten sollten Erwartungen und Grenzen für das gemeinsame Arbeiten und Lernen kommunizieren.

- Es ist von Bedeutung, dass ein Raum für soziale Begegnungen geschaffen wird.

- Die Hochschule sollte ermöglichen, dass in der Lehre ein Raum für Kollaboration entsteht.

- Ausreichend emotionale Unterstützungsstrukturen sollten vonseiten der Hochschule vorhanden sein.

Dimension Akteur ${ }^{\star}$ innen: studierendenzentriert denken

- Traditionelle Rollenbilder von Lehrenden und Lernenden sollten hinterfragt werden.

- Die Hochschule sollte Heterogenität in der Studierendenschaft wahrnehmen und einbeziehen.

- Es ist wichtig, dass die Hochschule die Möglichkeit zur Partizipation am Entwicklungsprozess einräumt. 
- Bei der Pfadentwicklung der Hochschule sollte die Kompetenzentwicklung der Studierenden in den Fokus genommen werden.

- Die Hochschule sollte so gestaltet sein, dass sie als Sozialisationsraum wirken kann.

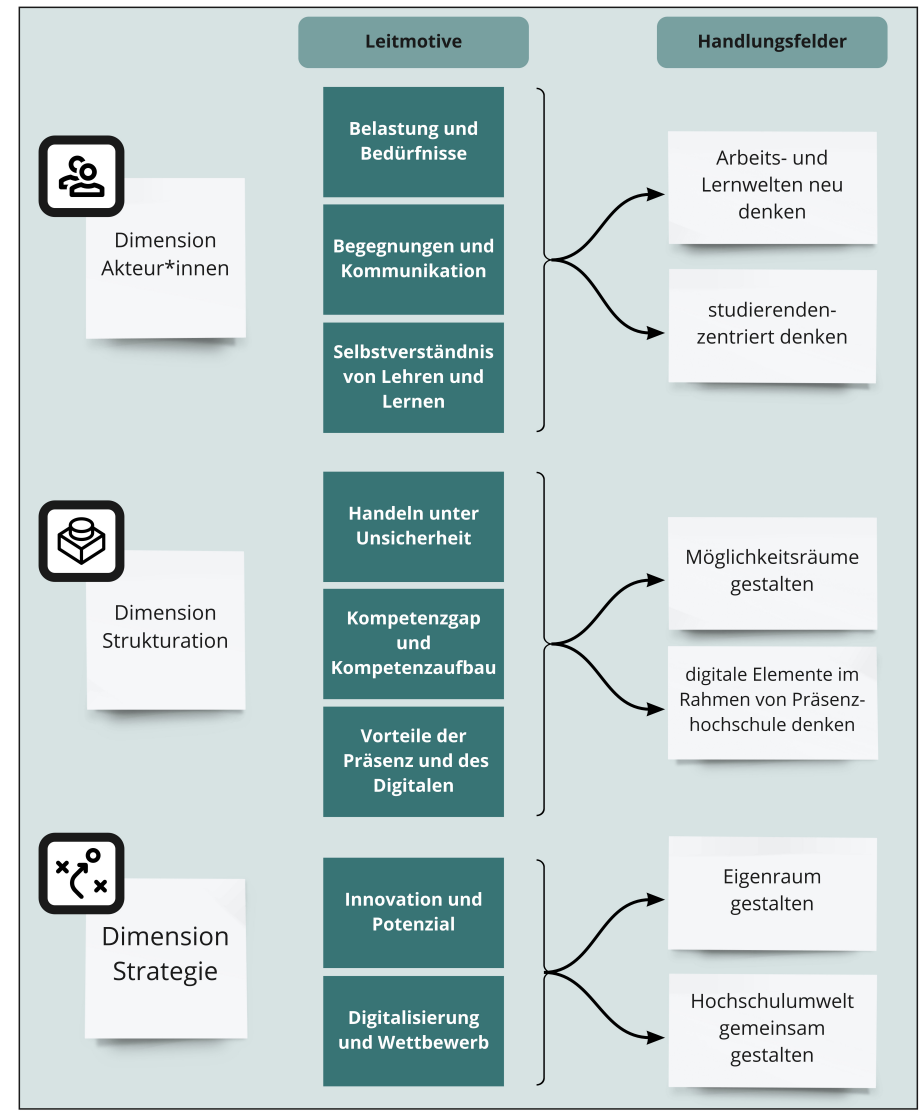

Abbildung 1: Ergebnisse der Studie

\section{Dimension Strukturation: Möglichkeitsräume gestalten}

- Bei der Pfadentwicklung sollten insbesondere die Fähigkeiten der Beteiligten fokussiert und die Anforderungen klar definiert werden. 
- Die Hochschule sollte als Ort der Gemeinschaftsbildung begriffen und entsprechend gestaltet werden, damit Identifikation möglich wird.

- In Entscheidungsprozessen sollte zukünftig Agilität möglich sein.

- Es ist von Bedeutung, dass die Hochschulen ihre Unterstützungsprozesse für die digitale Lehre weiter professionalisieren und ausbauen.

- Es sollten weitere Experimentierfelder eröffnet werden, um Neues im geschützten Raum auszuprobieren und auch Scheitern und erneutes Versuchen zu erlauben.

Dimension Strukturation: digitale Elemente im Rahmen von Präsenzhochschule denken

- Die Dualität im Denken entweder Digital oder Präsenz sollte aufgegeben werden.

- Es ist grundlegend, den Nutzen des Digitalen für Präsenzhochschulen sicherzustellen und zu thematisieren.

- Der Anteil an Präsenz ist zu gewichten, wenn man das Profil der Präsenzhochschule behalten will.

- Die während der Pandemie erprobte inkrementelle Arbeitsweise sollte beibehalten werden, um sich der Pfadentwicklung Stück für Stück zu nähern.

Dimension Strategie: Eigenraum strategisch gestalten

- Promotoren sollten innerhalb der Hochschulen identifiziert werden und eine Koalition bilden.

- Es ist relevant, hochschulinterne Barrieren zu identifizieren und Strategien zu deren Abbau zu entwerfen.

- Für eine strategische (Neu-)Ausrichtung ist ein strategischer Kern aufzubauen, der alle betroffenen Akteur*innengruppen einbezieht.

- Hochschulen sollten mögliche Pfade post Corona erkennen und sich Schritt für Schritt den eigenen Pfad erschließen.

Dimension Strategie: Hochschulumwelt gemeinsam gestalten

- Es sollte genug Freiraum für die selbstständige, strategische Positionierung von Hochschulen in der Bildungslandschaft geschaffen werden. 
- Es ist grundlegend, dass Hochschulen sich ergebende Autonomiegrade nutzen und selbst eine aktive Rolle einnehmen.

- Hochschulen sollten (gemeinsam) zukunftsweisende Studienmodelle entwickeln.

- Durch Kooperationen zwischen den Hochschulen ist die Aufgabe der strategischen Ausrichtung gestärkt zu bewältigen.

\section{Ausblick und Übertragbarkeit}

Mit der vorliegenden Studie ist es gelungen, mithilfe des qualitativen Ansatzes viele Einblicke in die Geschichten zu gewinnen, die sich an Hochschulen in den letzten beiden Semestern unter Corona-Bedingungen ereignet haben. Die Studie bietet wesentliche Erkenntnisse in verschiedenen Bereichen und kann als Entscheidungsimpuls für das Vorgehen bei der konzeptuellen Planung von Lehre an Hochschulen dienen. Dabei liefert sie einen Beitrag nicht nur auf didaktischer Ebene, sondern auch bei der grundsätzlichen Frage danach, welche Aspekte digitaler Lehre für Präsenzhochschulen im Sinne einer strategischen Weiterentwicklung zukunftsweisend sein können. Des Weiteren können die Ergebnisse der Studie unter Berücksichtigung der Lessons Learned herangezogen werden, um weitere strategiebildende Schritte im Prozess der Hochschul(weiter)entwicklung post Corona zu planen. Ebenfalls soll die Studie im bildungspolitischen Diskurs als Diskussionsgrundlage dienen, indem die Fragen fokussiert werden, was von den HAWs im Speziellen und von den Hochschularten im Allgemeinen nach der Pandemie übrig bleibt, welche zukünftigen Bedarfe Hochschulen haben und welche Hochschulprofile sich entwickeln werden. Ausgehend von den Erkenntnissen dieser Studie gibt es weiteren Forschungsbedarf hinsichtlich einer Beobachtung der Hochschulentwicklung im zeitlichen Verlauf. Insbesondere nach Wegfall der Corona-Auflagen sollte untersucht werden, inwiefern Hochschulen post Corona in der Lage sind, den Digitalisierungsschub in eine strategische Pfadentwicklung zu überführen und diesen durch eine Überwindung der Restauration in einen transformativen Prozess umzusetzen. 


\section{Ausgangssituation: digitale Lehre an Hochschulen}

Der Einsatz von digitalen Elementen ist keine Neuerung in der Hochschullehre post Corona ${ }^{1}$, sondern wird seit Jahrzehnten in einschlägigen bildungswissenschaftlichen Kreisen diskutiert. Was vor der Jahrtausendwende unter E-Learning verstanden wurde, hat sich in den Begriff der Digitalisierung gewandelt. Was derzeit während der Corona-Pandemie in aller Breite als „hybrid“ diskutiert wird, entspricht klassischerweise dem Begriff des Blended Learnings ${ }^{2}$. Die Vielfalt der Lehre und deren Kompetenzziele sowie der zum Teil von Grund auf verschiedene Habitus der Fachdisziplinen in Kombination mit der Spannbreite der am Prozess beteiligten Menschen und deren Haltungen verursachen, dass es auch in Hinsicht auf die Digitalisierung der Lehre kein als Königsweg zu titulierendes Vorgehen gibt. Was gute (auch digitale) Lehre ausmacht, wird vielfach diskutiert und kann zuletzt nicht einheitlich beantwortet werden. Was jedoch zumindest mit hinreichender Klarheit beantwortet werden kann, ist die Frage danach, welche Elemente digitaler Lehre ${ }^{3}$ nutzbringend und dementsprechend erhaltenswert oder entwicklungswürdig sind bzw. was sich nicht bewährt.

1 Die lateinische Präposition wird in der Studie nicht mit dem Akkusativ (post Coronam) verwendet, sondern im Zuge eines Lexikalisierungsprozesses und im Zusammengang mit „Corona” ohne Kasusrektion, wie in bereits vorliegenden die Pandemie betreffenden Veröffentlichungen. Gemeint ist ein Zustand nicht „nach dem Corona-Virus“, sondern "nach der Corona-Pandemie“.

2 Obwohl „Blended-Learning“ wissenschaftlich von seiner Herkunft und Verwendung her korrekt ist, wird im Folgenden der Begriff „hybrid“ aufgenommen, da er sich in den empirischen Daten mehrheitlich wiederfindet. „Hybrid“ ist in diesem Kontext noch nicht in allen Facetten definiert, in der Studie werden unter „hybrid“ alle Formen von Lehr-/Lernsettings verstanden, die sowohl Präsenz- als auch digitale Anteile umfassen.

3 Unter „digitaler Lehre“ werden in der vorliegenden Studie nach Kerres (2018) alle zeit-/ortsunabhängigen synchronen und asynchronen Formen des Lehrens und Lernens mit digitalen Medien subsumiert. Von den Interviewten wurde dies auch als Onlinelehre, E-Learning oder virtuelle Lehre bezeichnet. 
Digitalen Elementen in der hochschulischen Lehre werden viele Chancen, aber auch Hemmnisse zugesprochen. Ein zentraler Aspekt liegt auf dem Gesichtspunkt der Individualisierung des Lernens. Wenn sich Studierende nicht in einem Hörsaal einfinden und dieselbe neunzigminütige Vorlesung hören, sondern in einem Onlinesetting Lernangebote zur Verfügung gestellt werden, mag eine individuelle Förderung von Studierenden bzw. ein individuelles Lernen möglich sein. Zumindest bietet der Einsatz digitaler Werkzeuge grundsätzlich die Möglichkeit, solche individualisierten Lernangebote $\mathrm{zu}$ schaffen (Iberer \& Müller, 2002; Meier, 2006). So können verschiedene Lerntypen ebenso wie unterschiedliche Leistungsniveaus der Studierenden angesprochen werden. Studierende, die den Bedarf haben, durch vermehrtes Üben den Stoff zu wiederholen, können so ebenfalls gefördert werden wie Studierende, die der Gruppe voraus sind (Götzelt, 2010). Hier sind Lehrende in einer neuen Verantwortungsrolle, um dieses Potenzial des digitalen Settings zu erschließen.

Viele Beispiele zeigen, dass es hochwertige digitale Lehre an deutschen Hochschulen gibt. Es zeigt sich wiederum vielfach, dass ein Teil der Lehrenden nicht adäquat auf die Durchführung digitaler Lehr-/ Lernformate vorbereitet ist (Handke, 2020). Die dadurch entstehende Überforderung hat zum einen eine kapazitive Dimension. Eine Lehrveranstaltung im laufenden Betrieb neu aufzusetzen und mit entsprechend hochwertigen digitalen Elementen anzureichern, gleicht der Restauration eines Schiffes während der Fahrt auf hoher See und ist nebenbei kaum zu leisten. Nur selten können entsprechende Freiräume geschaffen werden, die es erlauben, Lehrveranstaltungen in einer entsprechenden Qualität neu aufzusetzen. Eine zweite Dimension betrifft die Kompetenz der Lehrenden hinsichtlich digitaler didaktischer Modelle. Hier ist ein Kompetenzdefizit zu beobachten (Mayert, 2017; Rohs et al., 2017). Es kann von Lehrenden an Hochschulen nicht als selbstverständlich erwartet werden, dass entsprechende mediendidaktische Kompetenzen vorliegen, um hochwertige Lehr-/Lernsettings aufzubauen. Lehrende sind darauf angewiesen, dass entsprechende Fortund Weiterbildungsmaßnahmen angeboten werden (Mayert, 2017; Richter, 2017; Sgier et al., 2018). Unterstützend wirkt, wenn der nötige Freiraum geschaffen wird, um diese Fortbildungsangebote wahrzunehmen und deren Inhalte in der Lehrrealität umzusetzen. Kapazitive 
Limitationen und der Bedarf, gezielte Kompetenzen aufzubauen, sind aber nicht allein die wesentlichen Gesichtspunkte. Maßgeblich entscheidend für die Umsetzung digitaler Lehr-/Lernformate auf der Seite der Lehrenden ist die persönliche Haltung (Bolten \& Rott, 2018). Eine positive Einstellung gegenüber digitalen Medien wirkt sich förderlich auf die Einstellung hinsichtlich digitaler Hochschullehre aus. Die Erfahrung zeigt, dass diese positive Haltung mit einer Innovationskraft einhergeht und - trotz hoher kapazitiver Belastung - die nötigen Kompetenzen erworben und unmittelbar in qualitativ hochwertige Lehr-/Lernsettings umgesetzt werden.

Ein weiterer positiver Aspekt von digitalen Lernangeboten ist die zeitliche und räumliche Flexibilität (Bargel, 2013; Kerres et al., 2012). So muss nicht zwangsläufig der Stundenplan vorgeben, wann welches Fach gelernt wird. Asynchrone Lernangebote machen es möglich, dass die Studierenden selbst entscheiden können, wie sie sich das Lernen einteilen. Es kann idealerweise gelernt werden, wenn die persönliche Aufnahmefähigkeit am höchsten ist (Busse, 2017). So kann es verschiedenen Persönlichkeitstypen ermöglicht werden, in einem selbstorganisierten Prozess und passgenau nach den eigenen Bedürfnissen zu studieren. Außerdem bieten digitale Lehr-/Lernsettings die Möglichkeit der räumlichen Entgrenzung. Unabhängig davon, ob es sich um synchrone oder asynchrone Formate handelt, sind die Studierenden nicht mehr darauf angewiesen, zu einem bestimmten Zeitpunkt an der Hochschule zu sein. Lernen ist dadurch an vielen Orten möglich: Sei es in der WG, im elterlichen Haus oder auf dem Campus. So wird Lernen automatisch barrierefrei hinsichtlich räumlicher Hemmnisse (Fisseler, 2021). Genau diese räumliche und zeitliche Flexibilität macht jedoch ein besonders hohes Maß an Eigenverantwortung und Organisation in Bezug auf den eigenen Lernprozess notwendig (Iberer \& Müller, 2002). Es ist eine große Herausforderung vor allem für Studienanfänger*innen, sich derart zu organisieren und die Gestaltung der Rahmenbedingungen des eigenen Lernens selbstbestimmt zu verantworten. Nicht nur der erzeugte Druck des Stundenplans, sondern auch die vorgegebene Struktur fällt weg. Wenn sich jede ${ }^{\star} r$ Studierende selbst organisiert und individuelle persönliche Strukturen anlegt, gehen zudem Schnittstellen zu Kommiliton ${ }^{\star}$ innen verloren, die ggf. in einem anderen Tempo oder einer ganz anderen Struktur studieren. 
Die vollständige Umstellung des Lehrbetriebs auf asynchrone Lehrformate ist in der Regel kein realistisches Szenario in der regulären Lehre. Im Bereich der Weiterbildung und des Fernstudiums sind Lehr-/ Lernstrukturen häufig durch lange (auch mediengestützte) Selbstlernphasen gekennzeichnet. Insbesondere in diesem Bereich wird ersichtlich, dass es eine Herausforderung darstellt, unter den Studierenden ein verbindliches Gefühl der Gemeinschaft herzustellen bzw. einzelne Studierende über das Semester hinweg nicht zu verlieren. Für den Sozialisationsprozess der Studierenden ist aber die Identifikation mit der Gemeinschaft sowie mit dem Fach ein wichtiger Faktor. Neben der Frage nach der Organisationsfähigkeit vonseiten der Studierenden ist ein weiteres Problem die Gefahr der Prokrastination sowie ein hohes Ablenkungspotenzial (Kindler et al., 2020). In einer Gruppe zieht das Gefühl des Miteinander-Lernens auch über manche motivationslose Phase hinweg. Gemeinsam lernt es sich ggf. konsequenter, weil die Gruppe einen Druck von innen heraus aufbaut. Alleine vor einem Bildschirm oder am Schreibtisch zu Hause fehlt das soziale Gefüge, was dazu führt, dass andere Betätigungsfelder gefunden werden. Anders als im Hörsaal oder im Seminarraum muss sich der/die Lernende disziplinieren und bewusst dafür entscheiden, in diesem Moment zu lernen. Ein Mangel an Selbstdisziplin kann zu einem prokrastinativen Verhalten führen und der Versprechung an sich selbst, dass die Aufgaben noch später erledigt werden können. Problematisch wird es, wenn dieses „später“ nicht kommt oder Lernstoff so lange aufgeschoben wird, bis sich der dadurch entstehende Berg unüberschaubar aufhäuft und dann (vermeintlich) nicht mehr bewältigt werden kann. Studierenden, die in einer Lernsituation auf sich alleine gestellt sind, fehlt allzu oft die Anbindung nicht nur an die Lehrenden, sondern auch an Kommiliton*innen. Sie sehen nicht, wie gut oder schlecht die anderen vorankommen. Es fehlt der soziale Referenzrahmen.

Diese Beobachtung kann jedoch auch ins Positive umgekehrt werden. Sind die Studierenden selbst für ihre Lernstruktur verantwortlich, dann kann es gelingen, dass sie wichtige Selbstlernkompetenzen aufbauen, die entscheidende Bausteine für Lebenslanges Lernen sind (Erpenbeck \& Sauter, 2019). Sehr abhängig vom jeweiligen Studiengang bzw. der Studienphase sind Lerngelegenheiten entweder eher selbstbestimmt oder verschult. Gerade durch die Modularisierung der 
Studiengänge kommt es häufig (im positiven Sinne) zu sehr klaren Studienstrukturen, die aber nicht selten das Studium vom ersten Semester bis zur Thesis vorzeichnen und nur wenig Freiraum geben. In einem solchen System ist es durchaus eine Herausforderung, die Studierenden in die Rolle zu bringen, selbst Verantwortung für ihren Lernfortschritt zu übernehmen. Flexibilisierte und individuellere digitale Lernumgebungen können dazu beitragen, dass Studierende eine eigenverantwortliche Rolle hinsichtlich der Gestaltung ihres Studiums einnehmen.

Ein räumlich und zeitlich individualisiertes Setting kann aber nicht nur dazu führen, dass der Lernfortschritt erheblich beeinflusst wird, sondern es fehlt auch die sozio-emotionale Begleitung der Lernenden und durch die Lehrenden (Apelojg et al., 2020). Im schlimmsten Fall können Studierende vereinsamen (Spitzer, 2014). Idealerweise begleiten Lehrende ihre Studierenden in einem Entwicklungsprozess, der über die reine Fachlichkeit hinausgeht. Dies geschieht oft implizit, indem der/die Lehrende seine/ihre Studierenden wahrnimmt und in seinen Handlungen auf die Situation der Gruppe oder auch des/der Einzelnen reagiert. In digitalen Lernbedingungen fehlt die motivationssteigernde, persönliche Kommunikation (Iberer \& Müller, 2002; Meier, 2006). Das Miteinander zwischen den Studierenden bzw. zwischen Studierenden und Lehrenden hat demnach nicht nur den Zweck des Aufbaus von fachlicher Kompetenz. Der persönliche Austausch sorgt dafür, dass die eigene Motivation, dem Studium zu folgen bzw. dieses im Idealfall selbst aktiv zu gestalten, steigt. Vor allem hinsichtlich motivationaler Faktoren sind dem digitalen Setting Grenzen gesetzt, die das persönliche Miteinander nicht kompensieren kann. Da der Studienerfolg jedoch mit Anwesenheit korreliert (Schulmeister \& Loviscach, 2017), scheint die soziale Einbettung der Studierenden sowie das Lernen miteinander eine wesentliche Rolle zu spielen.

In einem räumlich vom Campus der Hochschule entkoppelten Setting geht das verloren, was auf dem Campus scheinbar nebenbei geschieht. Traditionell gesehen sind die Hochschulen ein Ort der Begegnung, der weit über die rein fachspezifische Dimension hinausgeht. Dieser physische Ort hat nicht nur Einfluss auf den Lernfortschritt von Studierenden, sondern auch auf den Entwicklungs- und Sozialisationsprozess junger Menschen. An den Hochschulen erwerben sie einen (oft 
fachspezifischen) Habitus, der sich nicht selten in einem Zugehörigkeitsgefühl zu einer Gruppe manifestiert und über weite Lebensspannen trägt. Ob es ein Äquivalent im digitalen Raum geben kann, ist zu klären, zeichnet sich aber derzeit nicht ab.

Andererseits ist es im digitalen Rahmen möglich, mit Personen in Kontakt zu treten, die sich an weit entfernten Orten befinden (Erkens, 2005). Ohne Reiseaufwand können so im Rahmen der Lehre zum Beispiel Expert*innen eingeladen werden, zu denen die Studierenden sonst keinen Zugang hätten. Auch in Bereichen der Internationalisierung können digitale Kommunikationswege hilfreich sein. So können neben ganzen Auslandssemestern auch kleinere internationale Bausteine in ein Studium eingebaut werden. Dazu zählen auch die Vorund Nachbereitung von Auslandsaufenthalten.

Auch große Gruppen können mit digitalen Medien in der Lehre motiviert werden (Kreidl, 2011). Was in einem Hörsaal mit Studierendenzahlen im dreistelligen Bereich nicht gelingt, kann über digitale, interaktive Werkzeuge ermöglicht werden. Multimedial angereichertes Lernmaterial kann gezielt dazu eingesetzt werden, Verständnis zu fördern (Bremer, 2017; Handke \& Schäfer, 2012). Das kommt vor allem dadurch zustande, dass verschiedene Lerntypen mit unterschiedlichen Medien angesprochen werden können. Dieser Zusammenhang ist grundsätzlich für die Gestaltung von Lernmaterial bekannt, um nicht nur bestimmte Lerntypen zu erreichen (Creß, 2006; Vester, 2007)

Um diese Möglichkeiten digitaler Lehr-/Lernmethoden jedoch zu nutzen, ist ein verändertes Rollenverständnis auf der Seite der Lehrenden notwendig (Szczyrba, 2006). Ein traditionelles Bild der allwissenden und unnahbaren Professorenschaft ist längst überholt und wird auch nicht mehr oft gelebt. Über eine studierendenzentrierte Lehre hinaus ist jedoch die Veränderung der Lehrendenrolle, die durch die Digitalisierung hervorgerufen wird, weiterhin hochkomplex (Alexander et al., 2019). Lehrende werden vermehrt zu Lernbegleitenden, die Lerngelegenheiten für ihre Studierenden schaffen, die genau auf die jeweilige Gruppe der Studierenden passen. Die Grenze zwischen Lehrenden und Studierenden verschwimmt immer mehr (Cendon, 2018). Aufgrund dessen ist die Hochschuldidaktik stark individualisierbar. Die dazu geforderte Nutzung verschiedener Medien und die dazu notwen- 
dige Kompetenz stellen die Lehrenden vor eine weitere Herausforderung. Hemmnis ist dabei eine Furcht vor sich verändernder Lehrpraxis (Paepe et al., 2019). Lehrende aus digitalen Lehr-/Lernsettings berichten, dass sie sich vor einer Menge an anonymen „Briefmarken“ im digitalen Klassenzimmer gegenübersehen, ihre Studierenden nicht wahrnehmen können und Lehrsituationen als einseitig empfinden. Als Folge kann dies bei den Lehrenden zu einem Gefühl der Isolierung führen (Paepe et al., 2019). Um diesem Gefühl entgegenzuwirken, braucht es innovative digitale Lehr-/Lernformate, die Interaktion ermöglichen.

Idealerweise führt der Einsatz digitaler Lehre zu einem Aufbau der Medienkompetenz aufseiten der Studierenden (Bäder, 2020). Denn auch von den sogenannten Digital Natives, die die Nutzung von Messenger-Diensten und Social Media gewohnt sind, kann kein vergleichbar kompetentes Verhalten beim digitalen Lernen vorausgesetzt werden (Lermen, 2017; Persike \& Friedrich, 2016; G. Reinmann, 2017; Schulmeister \& Loviscach, 2017). Auch Studierende müssen in die Lage versetzt werden, die zur Verfügung gestellten Medien lernerfolgssteigernd zu nutzen. Lehrende und Hochschulen als Ganzes sind dafür verantwortlich, dass die Studierenden die Gelegenheit erhalten, die hier notwendige Kompetenz aufzubauen.

Der Einsatz digitaler Medien in der hochschulischen Lehre beinhaltet viele Chancen und Hemmnisse. Der hier gezeigte Abriss bietet nur einen kleinen Einblick in Aspekte der aktuellen Bildungsforschung und ist keine abschließende und vollständige Betrachtung. Dennoch zeigt er in einer ersten Näherung die Multidimensionalität digitaler Lehre. Im Rahmen der Situation während und post Corona stellt sich die Frage, wie diese Erkenntnisse dabei helfen, zukünftige Lehre zu gestalten.

Über die Argumente hinweg scheint es so zu sein, dass eine Absolution entweder der digitalen Lehre oder der Präsenzlehre nicht zielführend ist. Es stellt sich nicht die Frage, ob Digital oder Präsenz, sondern vielmehr muss geklärt werden, welche Formate digitaler Lehre in Kombination mit Präsenzlehre den Studienerfolg einerseits befördern und andererseits dazu führen, dass Studierende einen außerfachlichen Entwicklungsprozess durchlaufen können. 
Die Einführung und Durchführung von digitalen Lehr-/Lernformaten ist dabei mit vielen Hemmnissen verbunden. Eine Ad-hoc-Umstellung von Präsenzformaten auf digitale Formate scheint dabei nicht sinnvoll, denn es braucht ein erhebliches Maß an Kompetenzen (sowohl bei den Lehrenden als auch bei den Studierenden) und schließlich auch eine entsprechende Haltung, die den Einsatz digitaler Medien erst sinnvoll macht. Bis hierher ließe sich ableiten, dass es empfehlenswert ist, für die Umstellung von Präsenzformaten in hybride oder digitale Formate eine entsprechende Entwicklungszeit anzuberaumen, damit ebendiese Umstellung qualitativ hochwertig gelingt und die beteiligten Parteien in diesen Prozess einbezogen werden können.

Genau diese Idealsituation eines Entwicklungszeitraums für die Umstellung von Lehrformaten wurde durch die pandemiebedingte Situation komplett ausgehebelt: Im Frühjahr 2020 sahen sich die Hochschulen der Herausforderung gegenüber, ad hoc auf die Beschränkungen der COVID-19-Pandemie zu reagieren. Eine Lehre in Präsenz war nicht mehr möglich und der Vorlauf bis zum kommenden Semester betrug sehr wenige Wochen. Was eigentlich ein geplanter und konzentrierter Entwicklungsprozess hätte sein müssen, wurde nun innerhalb weniger Wochen erforderlich. Die Lehre wurde komplett digitalisiert und in ein "Emergency Remote Teaching” versetzt (Hodges et al., 2020). Dieser Vorgang war mit erheblichen Schwierigkeiten verbunden. Lehrende sahen sich vor der Herausforderung, Lehr-/Lernformate umzusetzen, die sie zuvor noch nie verwendet hatten. Studierende wurden in voneinander isolierte Situationen geworfen, die das Studium erheblich erschwerten. Für die Hochschulen für Angewandte Wissenschaften (HAWs), mit einem starken anwendungsbezogenen Schwerpunkt, war es eine besondere Herausforderung, diese Praxisorientierung weiter aufrechtzuerhalten.

Rückblickend auf die vergangenen zwei Semester ist wesentlich festzustellen, dass die Hochschulen vor vielen großen Herausforderungen standen. Gleichzeitig haben sich durch die Pandemie viele neue Möglichkeiten im Kontingenzraum aufgetan (s. Abbildung 2). Als Kontingenz wird die prinzipielle Offenheit bzw. Unsicherheit einer Person in der Umwelt beschrieben (Luhmann, 1987). Ein Kontingenzraum beschreibt einem Handlungsraum, in dem es mehrere Möglichkeiten gibt und damit das Ergebnis von Handlungen auch unterschiedlich sein 
kann. Gerade in der Corona-Krise entstand eine äußerst kontingente Handlungssituation, da der Weg in den Regelbetrieb verstellt war und nun der kontingente Raum der zu digitalisierenden Hochschule sich öffnete.

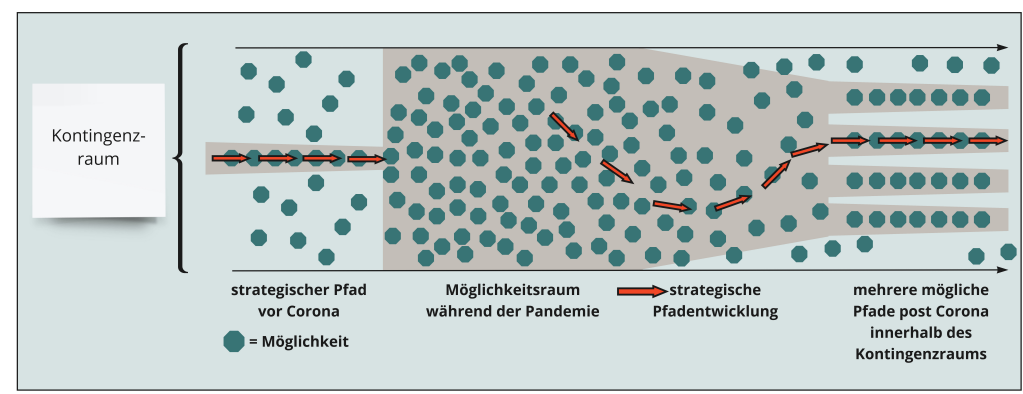

\section{Abbildung 2: Möglichkeitsraum}

Der Raum zwischen den waagrechten Pfeilen in Abbildung 2 verdeutlichen den Kontingenzraum. Die vor der Corona-Pandemie angelegten Hochschulstrategien, die in einem bestimmten Pfad innerhalb des Kontingenzraums verliefen, wurden durch den Ausbruch des Pandemiegeschehens vor eine große Herausforderung gestellt. Der Möglichkeitsraum (in der Abbildung grau hinterlegt) vergrößerte sich in seiner Bandbreite aufgrund der Notwendigkeit, agil auf die neuen Bedingungen zu reagieren. Die Möglichkeiten innerhalb des Kontingenzraums waren vielfältig und sind es noch. Dies bietet eine besondere Chance der Neuausrichtung der Hochschulen und stellt zugleich die Frage nach dem strategischen Pfad post Corona. Hier können Hochschulen ebenfalls verschiedene Entwicklungspfade einschlagen. In der Situation vor Corona waren die Hochschulen auf einem strategischen Pfad unterwegs, der durch klare Rahmenbedingungen als Begrenzung des Pfads gegenüber dem Kontingenzraum gekennzeichnet war. Neben dem schmalen Pfad (durch die Pfeile gekennzeichnet) waren weitere Möglichkeiten denkbar, lagen aber entweder außerhalb der Rahmenbedingungen oder waren nicht passfähig zur Hochschulstrategie. Mit Eintritt der Pandemiesituation fanden sich die Hochschulen in einem breiten Kontingenzraum mit vielen offenen Möglichkeiten 
wieder. Zu Anfang war dieser Möglichkeitsraum nur bedingt durch Rahmenbedingungen beschränkt und entsprach in seiner Breite dem Kontingenzraum als Ganzes. Im Rahmen dieses Raums haben die Akteur*innen trotz Belastungen unter Rückgriff auf ihre Fähigkeiten mündig durch Handlung Struktur erschlossen. In diesem Raum traten emergente, also nicht geplante, Strategien zutage. Diese emergenten Strategien, die innerhalb des Möglichkeitsraums auftraten, müssen nun in eine Pfadentwicklung post Corona umgesetzt werden (weiterführend s. Kapitel 7).

Die Hochschulen haben durch die Pandemie einen erheblichen Entwicklungsschub erhalten. Die Digitalisierung der hochschulischen Lehre wurde damit schneller vorangebracht als jemals gedacht. Die Hochschulen befinden sich auf dem Weg zur Digitalisierung, ebenso wie verschiedene Wirtschaftszweige auch (Gilch et al., 2019). Es stellt sich nun die Frage, in welche Richtung dieser Innovationsschub kanalisiert werden soll. Wie schafft es eine Hochschule von der in den letzten zwei Semestern ad hoc durchgeführten Restauration der Hochschullehre hin zu einer Pfadentwicklung, die sie in einen Transformationsprozess hineinträgt?

Viele Hochschulen haben Evaluationen genutzt, um den Entwicklungsstand $\mathrm{zu}$ beobachten (CHE Centrum für Hochschulentwicklung, 2020) und viele Forschungskontexte haben sich mit den Geschehnissen während der letzten Semester unter Corona-Bedingungen befasst (Schumacher et al., 2021; Seyfeli et al., 2020). Die hier vorgestellte Studie greift die bereits vorliegenden Erkenntnisse auf und überführt sie in einen qualitativen Forschungsansatz (s. Kapitel 3). Das folgende Kapitel gibt zunächst einen Einblick in einen Teil der bisher vorliegenden Studien. 


\section{Aktueller Stand: Studien zum Thema Lehre und Corona-Pandemie}

Wie bereits im ersten Kapitel geschildert, ist es eine der großen, zukunftsweisenden Aufgaben für Hochschulen, aus dem Stadium der im Corona-Betrieb vorgenommenen Restaurationen der Lehre eine Pfadentwicklung abzuleiten. Diese Entwicklung führt Hochschulen in einen transformativen Prozess, in dem sie sich selbst und damit das hochschulische Bildungssystem neu definieren. Ein wesentlicher Bestandteil eines transformativen Prozesses ist die Kenntnis über den Systemzustand. Viele Hochschulen haben sich mithilfe von Evaluationen genau mit ihrem Ist-Zustand im Corona-Setting befasst (CHE, 2020). Außerdem haben viele Forschungseinrichtungen den Faden aufgenommen und empirische Forschungsarbeiten in diesem Feld veröffentlicht (Schumacher et al., 2021; Seyfeli et al., 2020). Diese CoronaStudie knüpft an den bereits vorhandenen Forschungsstand an. Es ist ein wesentliches Ziel, die vorliegenden Studien sinnvoll zu ergänzen und in die Überlegungen hinsichtlich des qualitativen Forschungsdesigns (s. Kapitel 3) einzubeziehen. Daher ist eine Auseinandersetzung mit dem aktuellen Forschungsstand zur Corona-Situation an Hochschulen unbedingt geboten. In diesem Kapitel wird aufgrund dessen der aktuelle Forschungsstand im Hinblick auf die Auswirkungen der Corona-Pandemie auf die Hochschullandschaft ${ }^{4}$ dargestellt. Hierbei werden die wichtigsten Ergebnisse aus bereits veröffentlichten Studien (Stand 15.04.2021) zusammengefasst beschrieben. Im ersten Teil des Kapitels werden quantitative Studien erläutert, im zweiten Teil Studien qualitativer Art.

4 Da teilweise aus den Studien nicht ersichtlich wird, ob es sich um Befragungen an Universitäten, Pädagogischen Hochschulen oder Hochschulen für Angewandte Wissenschaften handelt, wird im Folgenden der Überbegriff der Hochschulen verwendet, außer es ist explizit erkennbar, dass es sich um Hochschulen für Angewandte Wissenschaften handelt. 


\subsection{Quantitativer Forschungshintergrund}

Die Corona-Pandemie ist für deutsche Hochschulen eine bisher ungekannte Ausnahmesituation. Bereits während des Sommersemesters 2020, also kurz nach Beginn der Corona-Pandemie, wurden verschiedenste Erhebungen und Studien durchgeführt, um die Sicht von unterschiedlichen Akteur*innengruppen (z. B. Hochschulleitungen, Studierenden oder Lehrenden) auf die Hochschulen während der CoronaPandemie zu untersuchen.

\subsubsection{Hochschulinterne Studien}

Die Perspektive von Studierenden auf die Corona-Semester wurde mittels Onlinebefragungen in einzelnen lokalen Studien in Deutschland erfasst. Besonders zahlreich sind die Erhebungen an einzelnen Hochschulen, die einer internen Orientierung über die Auswirkungen der Corona-Pandemie auf die eigene Institution dienen (vgl. dazu Boros et al., 2020a, 2020b; Feucht et al., 2020; Kindler et al., 2020; Meißelbach \& Bochmann, 2020; Pauli et al., 2020; Peters, 2020; Stammen \& Ebert, 2020). Wie diese Studien, behandelten auch die meisten anderen hochschulinternen Erhebungen insbesondere Herausforderungen eines digitalen Studiums bzw. retrospektive Bewertungen der Erfahrungen nach den ersten Corona-Semestern. In den verschiedenen hochschulinternen Studien tauchten Themen in den beiden Bereichen (1) entstehende Barrieren bei der Bildungsteilhabe sowie (2) Zeitbudget und Arbeitsaufwand wiederkehrend auf. Die pandemiebedingte unerwartete Umstellung auf ein digitales Semester führte so die abgeleiteten Befunde - zu Barrieren der Bildungsteilhabe bei einem Teil der Studierenden. Diese abgeleitete Erkenntnis lag unter anderem darin begründet, dass die technische Ausstattung der Studierenden längst nicht den Voraussetzungen für das digitale Studium entsprach. Es zeigte sich, dass ungefähr $30 \%$ der befragten Studierenden mindestens ein weiteres technisches Endgerät benötigten. Von den Studierenden waren mehr als drei Viertel ausreichend mit technischen Endgeräten ausgestattet, um an digitaler Lehre teilnehmen zu können (Peters, 2020; Stammen \& Ebert, 2020). Bei zwei Dritteln der befragten 
Studierenden reichte die Internetverbindung für synchrone Veranstaltungen nicht aus, sodass sie nicht daran teilnehmen konnten (Feucht et al., 2020). Auch die - durch das digitale Studium - nicht mehr verfügbare technische Infrastruktur der Hochschulen wurde in einigen Studien thematisiert. Für viele Studierende ist es unproblematisch, ohne Scanner, Drucker bzw. Kopierer in den Hochschulen auszukommen, für einen nicht unerheblichen Anteil (zwischen 20 und $30 \%$ der Befragten) stellte das Fehlen dieser Ressourcen allerdings eine erhebliche Beeinträchtigung im digitalen Semester dar (Stammen \& Ebert, 2020).

Ungeachtet der technischen Ausstattung zeigte sich, dass das Studium nicht mit dem gleichen Zeitbudget betrieben werden konnte. So gaben Studierende einerseits an, durch Sorgetätigkeiten, z. B. aufgrund von unvorhergesehener Kinderbetreuung oder der Betreuung einer pflegebedürftigen Person, ihr Zeitbudget für das Studium reduzieren zu müssen, und andererseits war ein deutlich höherer Arbeitsaufwand durch das Homestudying zu verzeichnen (Boros et al., 2020b; Kindler et al. 2020; Peters, 2020; Stammen \& Ebert, 2020; Universität Bielefeld, 2020). In der Studie von Kindler et al. (2020) konnte dazu festgestellt werden, dass der Arbeitsaufwand sich von 21,4 Stunden/Woche auf 33,5 Stunden/Woche erhöhte. Dazu stellten Feucht et al. (2020) fest, dass vor allem in Seminaren die vielen Abgaben - die jetzt die Präsenzsitzungen ersetzen - einen deutlich erhöhten Arbeitsaufwand darstellten. Durch diese Umstellung von Präsenz- auf digitale Lehre hat sich laut der Hälfte der befragten Studierenden der Leistungsdruck erhöht.

Die hochschulinternen Befragungen befassten sich des Weiteren mit den Fragen nach den Sorgen und Ängsten bzw. der Lebenssituation Studierender. Zum Zeitpunkt der Befragung waren die Antworten auf die Frage nach der eigenen Angst der Studierenden vor der CoronaPandemie breit gestreut. Eine kleinere Gruppe gab an, starke Angst vor der Corona-Pandemie zu haben (Pauli et al., 2020). Über die Hälfte der befragten Studierenden gab an, dass sie negative bzw. sehr negative Auswirkungen auf ihre psychische und emotionale Befindlichkeit durch die Corona-Pandemie erlebte (Pauli et al., 2020). Laut der Befragung von Boros et al. (2020b) empfanden die Studierenden höhere Belastungen durch das digitale Semester, da soziale Kontakte fehlten (52\%), die Selbststrukturierung bzw. -organisation schwieriger 
war (36\%) und der Stress als höher empfunden wurde (30\%). Laut den Befragungen der Universität Bielefeld (2020) und von Peters (2020) empfanden über $20 \%$ der Studierenden die Selbstorganisation im Homestudying als schwierig. Die Herausforderungen bestanden laut Kindler et al. (2020) darin, dass in der digitalen Lehre und in der aktuellen Krisensituation die Studierenden von anderen Dingen abgelenkt werden, gleichzeitig unterschiedliche Aufgaben erledigen müssen und sich Sorgen um Gesundheit, Angehörige und gesellschaftliche Entwicklungen machten. Diese Herausforderungen ließen sich durch eine gute Onlinedidaktik nur bedingt auffangen. Studierende berichteten dazu, dass Studieren durch die digitale Lehre unpersönlicher wurde und der Austausch mit Kommiliton*innen in digitaler Form nur teilweise oder gar nicht gelang (Feucht et al., 2020; Pauli et al., 2020; Peters, 2020; Stammen \& Ebert, 2020; Universität Bielefeld, 2020). Des Weiteren fühlten sich die Studierenden vor allem durch E-Mails der Lehrenden und Universitäts-Newsletter gut über die veränderten Bedingungen informiert (Boros et al., 2020b; Universität Bielefeld, 2020).

Im Hinblick auf die Perspektive der Lehrenden gibt es bis zum jetzigen Zeitpunkt bedeutend weniger quantitative Studien. Eine Kurzbefragung der Lehrenden von Boros et al. (2020a) fokussierte dabei beispielsweise die Themen Veränderung der Lehre durch die Corona-Pandemie, die Umsetzung der digitalen Lehre und die Zufriedenheit damit. Insgesamt nahmen an der Studie über 600 Lehrende teil. Von den Lehrenden, die im Corona-Semester digitale Lehre durchführten, gaben mehr als $70 \%$ an, die persönliche Umstellung als groß bzw. sehr groß empfunden zu haben. Den zeitlichen Aufwand für die Gestaltung der Vorlesungen bzw. Seminare beurteilten über $80 \%$ der Lehrenden als (sehr) hoch und schätzten damit ihren Arbeitsaufwand im Vergleich zu vorherigen Semestern als (viel) höher ein (Boros et al., 2020a; Reinmann et al., 2020). Als besonders belastende Faktoren wurden von den Lehrenden die fehlenden sozialen Kontakte (45\%), die Anpassungsschwierigkeiten an neue Lehrformate (43\%) sowie Schwierigkeiten bei der Work-Life-Balance (25\%) genannt. Die Faktoren gesundheitliche Schwierigkeiten bzw. psychische Belastung und die fehlende Motivation wurden von jeweils $7 \%$ der Befragten am seltensten genannt. $\mathrm{Zu}$ der Einschätzung der digitalen Kompetenzen der Lehrenden entstand insgesamt ein uneinheitliches Bild: $75 \%$ der Lehrenden schätzten ihre 
digitalen Kompetenzen als (sehr) gut ein, obwohl nur $30 \%$ angaben, über sehr gute bzw. gute Vorerfahrungen im Bereich digitaler Lehre zu verfügen. Dazu konnten Reinmann et al. (2020) feststellen, dass $21 \%$ der befragten Lehrenden vor der Pandemie bereits Erfahrungen mit digitaler Unterstützung der Lehre hatten. Dieses Ergebnis widerspricht zum Teil dem in Kapitel 1 dargestellten und empirisch belegten Defizit von Lehrenden hinsichtlich mediendidaktischer Kompetenzen. Des Weiteren wurde die technische Infrastruktur aus Sicht der Lehrenden als defizitär wahrgenommen, und die fehlende Kommunikation führte laut der Studie von Boros et al. (2020a) zu großer Frustration.

\subsubsection{Hochschulübergreifende und nationale Studien}

Neben den hochschulinternen Erhebungen wurden im vergangenen Sommersemester auch einige hochschulübergreifende Studien durchgeführt. Zu nennen ist hier unter anderem das Projekt „Studieren in Deutschland zu Zeiten der Corona-Pandemie" des Deutschen Zentrums für Hochschul- und Wissenschaftsforschung (DZHW) in Zusammenarbeit mit der Arbeitsgruppe Hochschulforschung an der Universität Konstanz, in dessen Rahmen bundesweit über 25.000 Studierende von 23 ausgewählten deutschen Hochschulen zu ihrer Studienund Lehrsituation befragt wurden. Die Daten dieser Onlinebefragung wurden themenspezifisch ausgewertet. Auf dem Datensatz der DZHW basierend, zeigte sich in der Studie von Lörz et al., (2020), dass die Mehrheit der befragten Studierenden ihre Lehrveranstaltungen besuchen konnte, die für das Sommersemester 2020 geplant waren (74\%). Nur bei wenigen Studierenden fielen alle $(0,5 \%)$ oder ein Großteil (ungefähr $5 \%$ ) der Lehrveranstaltungen aus. Die Wissenschaftler ${ }^{*}$ innen leiteten daraus ab, dass es den Hochschulen gelungen ist, ihr Lehrprogramm kurzfristig umzustellen und für Studierende zugänglich zu machen (Lörz et al., 2020). Im Vergleich zu der Befragtengruppe aus der hochschulinternen Befragung wurde hier das Fehlen technischer Infrastruktur generell als weniger großes Problem bewertet. Es konnte festgestellt werden, dass die meisten Studierenden (78\%) über eigene Computer verfügten, die für digitale Lehre geeignet sind, jedoch erwies sich die Internetverbindung in Teilen (für $22 \%$ der Befragten) 
als problematisch. Auch war die Wohnsituation bei einem Teil der Befragten für die Teilnahme an digitalen Veranstaltungen nicht optimal: Jede ${ }^{\star} r$ fünfte Studierende gab an, dass die Wohnsituation nicht für digitale Lehre geeignet sei (Lörz et al., 2020). Die Ergebnisse der Pilot-Studie von (Marczuk et al., 2021) deuten darauf hin, dass sich die Studiensituation aus der Perspektive der Studierenden insgesamt verschlechtert hat. Dies betraf im Hinblick auf die Studiensituation insbesondere das Aufrechterhalten oder Knüpfen von Kontakten zu Kommiliton ${ }^{\star}$ innen $(86 \%)$ und den Austausch in Lerngruppen (77\%). Die individuelle Lernsituation wird laut den Befragten ebenfalls als schwieriger eingeschätzt - sowohl die Entwicklung passender Lernstrategien (52\%), die Tagesstrukturierung (62\%) als auch die Bewältigung des Lernstoffs (62\%) (s. dazu auch Winde et al., 2020). Im Vergleich zu der Befragtengruppe aus der hochschulinternen Befragung wurde hier das Fehlen technischer Infrastruktur generell als weniger großes Problem bewertet. Es konnte festgestellt werden, dass die meisten Studierenden (78 \%) über eigene Computer verfügten, die für digitale Lehre geeignet sind, jedoch erwies sich die Internetverbindung in Teilen (für $22 \%$ der Befragten) als problematisch. Auch war die Wohnsituation bei einem Teil der Befragten für die Teilnahme an digitalen Veranstaltungen nicht optimal: Jede ${ }^{\star} r$ fünfte Studierende gab an, dass die Wohnsituation nicht für digitale Lehre geeignet sei (Lörz et al., 2020). Die Ergebnisse der Pilot-Studie von (Marczuk et al., 2021) deuten darauf hin, dass sich die Studiensituation aus der Perspektive der Studierenden insgesamt verschlechtert hat. Dies betraf im Hinblick auf die Studiensituation insbesondere das Aufrechterhalten oder Knüpfen von Kontakten zu Kommiliton*innen (86\%) und den Austausch in Lerngruppen (77\%). Die individuelle Lernsituation wird laut den Befragten ebenfalls als schwieriger eingeschätzt - sowohl die Entwicklung passender Lernstrategien (52\%), die Tagesstrukturierung (62\%) als auch die Bewältigung des Lernstoffs (62\%) (s. dazu auch Winde et al., 2020).

In einzelnen Aspekten hat sich die Lernsituation hingegen vereinfacht: Einige Studierende (ungefähr $24 \%$ ) konnten im digitalen Semester ihren Tag besser strukturieren oder den Veranstaltungen besser folgen als vor der Corona-Pandemie (Marczuk et al., 2021). Es zeigte sich des Weiteren, dass bestimmte digitale Lehrformate gegen die erschwerte 
Studiensituation wirkten. Dies traf vor allem auf Videokonferenzen und abrufbare Videos zu. Während Videokonferenzen vor allem die Kontakte förderten, ermöglichten Videoaufzeichnungen insbesondere die individuelle Gestaltung der Lehrsituation (Marczuk et al. 2021). Zu der Zufriedenheit mit erreichtem Wissen und Können der Studierenden konnte ein ambivalentes Ergebnis festgestellt werden: $41 \%$ der Studierenden waren mit ihrem erreichten Wissen und Können (eher) zufrieden, wohingegen $28 \%$ der Studierenden (eher) nicht zufrieden waren. Dazu konnte herausgefunden werden, dass die Zufriedenheit der Studierenden mit den digitalen Lehrformaten zusammenhängt. Insbesondere Videokonferenzen und im Nachhinein abrufbare Videoaufzeichnungen förderten eine höhere Zufriedenheit mit dem erreichten Wissen und Können (Marczuk et al., 2021). Zu der finanziellen Situation der Studierenden untersuchten (Becker \& Lörz, 2020) mit dem DZHW-Datensatz mögliche Auswirkungen auf das Studium. Es wurde festgestellt, dass sich bei $21 \%$ der Befragten die Erwerbssituation verschlechtert hat, da sie entweder entlassen (10\%) oder unbezahlt freigestellt (14\%) bzw. die Arbeitszeit reduziert wurde. Dadurch reduzierten sich die monatlichen Einnahmen der Studierenden. Zur Überbrückung finanzieller Engpässe wurden seitens der Studierenden entweder Ersparnisse oder private Finanzierungsquellen, wie beispielsweise die Eltern, Verwandte oder Partner, genannt (Becker \& Lörz, 2020).

An einer Mixed-Methods-Studie (für einen Einblick in den qualitativen Teil der Studie s. Kapitel 2.2) des Forschungs- und Innovationslabors Digitale Lehre beteiligten sich zudem rund 18.500 Studierende und 2.800 Lehrende der bayrischen Hochschulen für Angewandte Wissenschaften, um ihre Sicht auf Studium und Lehre während der Corona-Pandemie darzulegen (Kreulich et al., 2020). Die Ergebnisse der Studierendenbefragung deuteten darauf hin, dass Studierende ihre digitalen Kompetenzen als gut einschätzten. Dazu ist erwähnenswert, dass Studierende der Sozialwissenschaften ihre digitalen Kompetenzen signifikant $(p<.01)$ schlechter einschätzten als Studierende aller anderen Fachbereiche. Die Studiengänge MINT und Wirtschaftswissenschaften schätzten sich im Vergleich positiver ein (Kreulich et al., 2020). Als größter Vorteil der digitalen Lehre nannten die Studierenden die örtliche und zeitliche Flexibilität und die Möglichkeit, das 
Lerntempo auf eigene Bedürfnisse anzupassen (z. B. durch Videoaufzeichnungen von Lehrveranstaltungen). Auch Wissenschaftler*innen der Universität Hildesheim (Traus et al., 2020) befragten in einer Studie bundesweit rund 3.500 Studierende zu ihren Erfahrungen und Perspektiven während der Corona-Pandemie. Die Studie konnte die Ergebnisse von bisherigen hochschulinternen Untersuchungen zu den Ängsten und Sorgen bestätigen (vgl. Boros et al., 2020b; Pauli et al. 2020; Peters et al., 2020; Universität Bielefeld, 2020). Studierende gaben sehr häufig an, dass sie sich Sorgen machten und nicht mehr, wie bisher, auf das Studium fokussiert waren. Andere Verpflichtungen beeinträchtigten die Situation im Homestudying. Laut den Wissenschaftler*innen deutete vieles darauf hin, dass sich für die Studierenden die Konzentration auf einen Lebensmittelpunkt, der gleichzeitig Lernort und Lebensort war, als nicht leicht darstellte. Die Selbstorganisation und die eigenverantwortliche Zeitplanung wurden von über $40 \%$ der Befragten dabei ebenfalls als eher schwierig beschrieben. Es wurde außerdem erkennbar, dass es durchaus einen Anteil unter den Studierenden gab, die das digitale Corona-Semester als Entlastung empfanden (Traus et al., 2020). Es antworteten jedoch deutlich mehr Studierende, dass sie sich belastet fühlten. Die körperliche Belastung durch die Pandemie fiel weniger stark ins Gewicht als die seelische Belastung. Auch wurde anhand der Antworten deutlich, dass die finanziellen Unsicherheiten aufgrund der Corona-Pandemie von den Studierenden als besonders belastend empfunden wurden. Das Gesamtbild zu den negativen Einschätzungen während des digitalen Semesters unterstrich, dass insbesondere psychosoziale Aspekte als belastend empfunden wurden und das Corona-Semester kennzeichneten. Viele der befragten Studierenden fühlten sich unter Druck gesetzt und machten sich Sorgen um die gesellschaftliche Situation in Deutschland.

Im Hinblick auf die Perspektive der Lehrenden zeigte sich laut Kreulich et al. (2020), dass ungefähr die Hälfte der Lehrenden bereits vor der Corona-Pandemie Erfahrungen in digitaler Lehre vorweisen konnte und $14 \%$ mehr als fünf Jahre Erfahrung im Einsatz digitaler Lehre hatten. Dazu wurde ein positiver Zusammenhang zwischen allgemeiner Lehrerfahrung und digitaler Lehrerfahrung festgestellt $(r=0.22, p$ $<.01)$ - je höher die allgemeine Lehrerfahrung, desto höher die digitale Lehrerfahrung (Kreulich et al., 2020). Die Motivation der Lehrenden, 
sich medientechnisch sowie didaktisch auszuprobieren bzw. sich fortzubilden, wurde laut Kreulich et al. (2020) als hoch eingeschätzt. Des Weiteren konnte festgestellt werden, dass Lehrende mit Erfahrung in digitaler Lehre signifikant motivierter waren, sich medientechnisch ( $p$ $<.05)$ sowie didaktisch $(p<.01)$ auszuprobieren. Laut Winde et al. (2020) standen $75 \%$ der befragten Lehrenden aller Fachrichtungen digitaler Lehre positiv gegenüber. Zudem gaben $52 \%$ der Lehrenden an, dass ihre Hochschule seit der Corona-Pandemie über digitale Lehrkonzepte verfügte. Vor der Corona-Pandemie wurde dies lediglich von knapp 20 \% der Befragten angegeben (Winde et al., 2020).

Der Diskurs rund um die Corona-Pandemie fokussierte zu Beginn zumeist die Perspektive von Studierenden und Lehrenden und ließ die Sichtweise der Hochschulleitungen unbeleuchtet. Aufgrund dessen initiierte das HIS-Institut für Hochschulentwicklungen eine Ad-hocStudie „Corona@Hochschule“, eine bundesweite Befragung von Hochschulleitungen, in der die Sichtweisen, Planungsstände und Entscheidungshintergründe fokussiert wurden (Bosse et al., 2020). Über $50 \%$ der Befragten gaben bei technischen und didaktischen Kompetenzen und Supportangeboten für Lehrende einen sehr hohen Bedarf bzw. einen grundlegenden Bedarf an Neuentwicklungen an, während bezüglich der technischen und lernbezogenen Kompetenzen der Studierenden der geringste Entwicklungsbedarf gesehen wurde. Die Umstellung auf digitale Lehrangebote wurde von über $80 \%$ der Befragten als vollkommen bzw. überwiegend gelungen eingestuft. Die größten Erfolge bei der Umstellung auf digitale Lösungen wurden im Bereich der Prüfungs- und Studienorganisation gesehen, ähnlich wie bei der Gremienarbeit und in Bezug auf Kommunikations- und Entscheidungsprozesse. $\mathrm{Zu}$ den Bereichen, in denen die Umstellung als weniger gelungen bewertet wurde, gehören insbesondere die Serviceleistungen für Studierende, die Rechtssicherheit der Studien- und Prüfungsorganisation sowie die technische Ausstattung von Studierenden, Lehrenden und dem Verwaltungspersonal. Noch in Planung befanden sich zum Zeitpunkt der Befragung vor allem Betreuungskonzepte für den Studieneinstieg, gefolgt vom Ausbau der technischen Infrastruktur und Raumnutzungskonzepten. Hinzu kam vielerorts auch die Planung von Unterstützung für Lehrende und von Serviceleistungen für Studierende. Zur Kommunikation von Informationen und Entscheidungen kamen insbesonde- 
re die Hochschulwebseite und E-Mails zum Einsatz, während Social Media, Videobotschaften oder Live-Streams eine (deutlich) geringere Rolle spielten (Bosse et al., 2020).

\subsubsection{Internationale Studien}

Neben hochschulinternen und hochschulübergreifenden bzw. nationalen Studien wurden ebenfalls internationale Studien durchgeführt. Internationale Studien zu den Auswirkungen der Corona-Pandemie auf die Situation junger Menschen (vgl. OECD, 2020) konnten feststellen, dass die Medienausstattung an Hochschulen im internationalen Vergleich stark differiert. Deutschland liegt dabei über dem OECD-Durchschnitt, im europäischen Vergleich aber im unteren Drittel (OECD, 2020). Daraus wurde abgeleitet, dass im Bildungssektor ein struktureller Digitalisierungsrückstand besteht, der sich auf die Situation der Studierenden insbesondere in den Corona-Semestern auswirkte. Darüber hinaus beschäftigen sich zahlreiche international vergleichende Studien mit der Situation Studierender, aber auch mit expliziter Ausrichtung auf internationale Aspekte des Hochschullebens, wie z. B. die Frage nach der Auswirkung der Corona-Pandemie auf die Studierendenaustausche (vgl. Gabriels \& Benke-Åberg, 2020; Kercher \& Plasa, 2020). Es zeigte sich beispielsweise, dass an knapp zwei Dritteln der befragten Hochschulen internationale Studierende im Sommersemester aufgrund der Reisebeschränkungen nicht wie geplant das Studium in Deutschland beginnen bzw. fortsetzen konnten. Die befragten Hochschulen (40\%) gaben zudem an, dass internationale Studierende Deutschland verließen, nachdem an den Hochschulen Maßnahmen oder andere Reisebeschränkungen in Kraft traten. Hochgerechnet ergab sich daraus in Deutschland eine Anzahl von ungefähr 80.000 Studierenden, die Deutschland aufgrund der Corona-Pandemie verlassen hat (Kercher \& Plasa, 2020).

Einzelne internationale Studierendenbefragungen, wie der International COVID-19-Student Well-being Survey (C19 ISWS), befassten sich explizit mit der Gruppe der Studierenden während der Corona-Semester. Im Fokus dieser internationalen Querschnittstudie standen hierbei die Veränderungen der Lebensbedingungen, des Wohlbefindens, des 
Gesundheitsverhaltens sowie der Studienbedingungen Studierender durch die Corona-Pandemie. Insgesamt nahmen über 130.000 Studierende aus 26 Ländern (meist europäische Länder) daran teil, darunter auch Studierende aus deutschen Hochschulen (vgl. Busse \& Zeeb, 2020).

Wie bereits durch eine nationale Befragung belegt (Becker \& Lörz, 2020), hat sich die finanzielle Lage der Studierenden pandemiebedingt verschlechtert. Dazu wurde im C19 ISWS angegeben, dass rund $16 \%$ der deutschen Stichprobe (Standort Bremen mit mehr als 1.800 Studierenden) derzeit über keine ausreichenden finanziellen Mittel verfügt (Busse \& Zeeb, 2020). Im Rahmen der C19 ISWS-Ergebnisse der belgischen (rund 26.000 Studierende) und der griechischen (rund 800 Studierende) Stichproben zeigte sich ein ähnliches Befundmuster: Hier waren es $14 \%$ der belgischen Studierenden (van de Velde et al., 2020) und über $18 \%$ der griechischen Studierenden (Stathopoulou et al., 2020).

Des Weiteren wurden darin die Studierenden befragt, ob die Veränderung der Lehrmethode coronabedingt erheblichen Stress verursachte: Knapp $50 \%$ der deutschen, über $43 \%$ der griechischen und über $73 \%$ der belgischen Stichprobe stimmten dieser Aussage (eher) zu. Über die Hälfte der befragten deutschen Studierenden berichteten von einem Anstieg des Arbeitsaufwands seit dem Pandemiegeschehen. Über $65 \%$ der befragten belgischen und ungefähr $46 \%$ der griechischen Studierenden gaben an, dass der Arbeitsaufwand seit der Corona-Pandemie (eher) zugenommen hat. Dieser Befund konnte bereits in bisherigen Studien festgestellt werden (Boros et al., 2020b; Kindler et al., 2020; Peters, 2020; Stammen \& Ebert, 2020; Universität Bielefeld, 2020). Es zeigte sich ebenso, dass Erstsemester mehr Stress und eine geringere Zufriedenheit als höhere Semester aufwiesen (van de Velde et al., 2020).

Zur Erfassung des Wohlbefindens wurden Studierende gebeten, Aussagen zu bewerten. Dabei sollten sie angeben, inwieweit sie diese Gefühle in der Woche vor der Befragung erlebt bzw. nicht erlebt hatten. Von einem Drittel der belgischen Studierenden wurde angegeben, dass sie sich meistens bzw. (fast) immer (32\%) einsam fühlten (van der Velde et al., 2020). Die griechische Stichprobe zeigte ein ähnliches 
Befundmuster mit knapp $28 \%$ (Stathopoulou et al., 2020). Fast die Hälfte (49\%) der belgischen und über $30 \%$ der griechischen Studierenden fühlten sich in der Corona-Pandemie isoliert und $45 \%$ der griechischen und über $58 \%$ der belgischen Studierenden vermissten die Gemeinschaft mit anderen. Lediglich knapp $14 \%$ der belgischen und $20 \%$ der griechischen Studierenden berichteten, dass sie keine Gesellschaft vermissten (Stathopoulou et al., 2020; van der Velde et al., 2020). Es wurde von van de Velde et al. (2020) festgestellt, dass mehr als $40 \%$ der Studierenden in der Corona-Zeit meist oder (fast) immer unruhig schliefen. Eine fast gleich große Gruppe berichtete, dass sie sich antriebslos fühlte sowie alles eine Anstrengung bedeutete. Außerdem gaben über $27 \%$ der Studierenden an, dass sie sich traurig und deprimiert fühlten, das Leben beinahe nicht genossen (18\%) und sich (fast) nicht glücklich fühlten (8\%) seit dem Ausbruch der CoronaPandemie. In der griechischen Stichprobe (Stathopoulou et al., 2020) zeigte sich, dass für über $40 \%$ der Studierenden alles eine Anstrengung war, während $33 \%$ meistens oder (fast) immer über depressive Symptome berichteten. Weniger als $30 \%$ der Studierenden gaben an, dass sie sich einsam oder traurig fühlten (24\%) oder dass sie meistens oder (fast) immer unruhig schliefen (26\%). Zudem wurde von $19 \%$ der Studierenden angegeben, dass sie (fast) nie das Leben genossen, wobei über $8 \%$ berichteten, dass sie sich in der letzten Woche (fast) nie glücklich fühlten (Stathopoulou et al., 2020).

Die Vielzahl an quantitativen Untersuchungen zeigt, dass es eine hohe Relevanz gibt, die Situation in dem Pandemiegeschehen näher zu beleuchten. Augenscheinlich können durch die Erhebungen quantitativer Art allgemeine Ableitungen zum Erleben von Studierenden, Lehrenden und Hochschulleitungen in der Corona-Pandemie gemacht werden. Hier sticht heraus, dass viele Herausforderungen nicht nur auf einer technisch-infrastrukturellen Ebene liegen, sondern auch persönliche Lebensbereiche und das Studieren als Lebensphase betreffen. Um einen detaillierten Einblick in Handlungsstrategie und den Umgang mit der Pandemiesituation zu gewinnen, sollen nun Ergebnisse aus qualitativen Forschungsansätzen dargestellt werden. 


\subsection{Qualitativer Forschungshintergrund}

Die qualitativen Studien, die in wie erwartet zum jetzigen Zeitpunkt in geringerer Anzahl vorliegen als die bereits beschriebenen Studien quantitativer Art, sind geeignet, um ein differenziertes Bild der CoronaSemester an Hochschulen aufzuzeigen.

\subsubsection{Hochschulübergreifende und nationale Studien}

Seyfeli et al. (2020) führten eine Studie mit synchronen und asynchronen Befragungen in Form von Chats und Videokonferenzen in einem zweiwöchentlichen Rhythmus mit Studierenden und Lehrenden von Hochschulen in Deutschland durch. $\mathrm{Zu}$ der pandemiebedingten Situation von Studierenden in den Corona-Semestern konnte festgestellt werden, dass die zeitliche und örtliche Flexibilität, die einsetzende Digitalisierung der Lehre durch die Corona-Pandemie sowie die Motivation der Lehrenden, digitale Lehre durchzuführen, als positiv eingeschätzt wurde. Jedoch wurden auch negative Aspekte der CoronaSemester genannt. „Der Verlust eines geregelten Tagesablaufes habe zu Schwierigkeiten mit der Selbstmotivation geführt" (Seyfeli et al., 2020, S. 3). Lernmotivation und die Leistung der Studierenden gingen aufgrund des fehlenden Austauschs und des fehlenden physischen Peerto-Peer-Lernens in den Corona-Semestern zurück. Zusätzlich sei der Workload gestiegen, weil die Prüfungsleistungen aufgrund der CoronaPandemie verändert wurden. Die von Seyfeli et al. (2020) befragten Studierenden berichteten selbst nicht über finanzielle Schwierigkeiten, jedoch berichteten sie von Kommiliton*innen mit eben diesen. Des Weiteren wurde von Seyfeli et al. (2020) gezeigt, dass einerseits die Planungssicherheit bzgl. der Prüfungen und Klausuren und andererseits der Einbezug der Studierenden in die funktionierende Umstellung der Hochschulen auf digitale Lehre gefehlt haben (Seyfeli et al., 2020). Die Gesamtbilanz der Studierenden fiel folgerichtig ambivalent aus.

Die Situation der Lehrenden betrachteten die Autor*innen der AutoEthnographischen Forschung zu digitaler Lehre und deren Begleitung, welche sich aus Lehrenden an deutschen Hochschulen zusammensetzen, die ihre eigene Lehrpraxis im ersten Corona-Semester beforsch- 
ten (Autor:innengruppe AEDiL, 2021). Kooperation in Form von Austausch über die eigene Lehre wurde von allen Teilnehmenden der Studie als unterstützend und gewinnbringend für die eigene Lehrpraxis empfunden. Das zentrale Ergebnis der Studie ist „das bewusste $\mathrm{Zu}$ lassen von Unsicherheiten“ (Autor:innengruppe AEDiL, 2021, S. 262), durch das eine Weiterentwicklung der Hochschullehre ermöglicht werde. Weitere Ergebnisse waren der gestiegene Workload, der auf die individuelle Lebenssituation während der Corona-Pandemie zurückzuführen war, die herausfordernde Kommunikation und Interaktion sowie die individuelle Ausgangslage in Bezug auf die eigene Medienkompetenz, die abhängig von der biografischen Erfahrung der Lehrenden mit Hard- und Software war. Auch Seyfeli et al. (2020) identifizierten die Motivation der Lehrenden zur Weiterentwicklung ihrer Lehre. Ein weiterer Aspekt, der in dieser Studie festgestellt wurde, war die Rollenveränderung der Lehrenden, da sie durch die digitale Lehre nun mehr Lerncoach als Wissensvermittler waren.

Eine weitere qualitative Studie hatte zum Ziel, Fachspezifika einzelner Fächercluster herauszuarbeiten. Hierfür befragte Bosse (2021) mit Leitfadeninterviews zehn Mitglieder von Fakultätsleitungen von je fünf Universitäten und Hochschulen für Angewandte Wissenschaften aus acht Bundesländern. In der fallübergreifenden Ergebnisdarstellung wurden Gemeinsamkeiten der Fakultäten, deren Leitungen befragt wurden, herausgearbeitet. Gemeinsamkeiten waren, dass die digitale Lehre von gesamthochschulischen Infrastrukturen profitierte und eine eigene Ausstattung der Fakultät von Vorteil war. Einzelne Fakultäten nutzten die eigenen Informatik-Fachkompetenzen, um die digitale Lehre zu verbessern, schufen Didaktik-Stellen und warben Fördermittel ein (Bosse, 2021). Im Umgang mit der Krisensituation wurden die Vorerfahrung sowie der persönliche Einsatz der Lehrenden als Gemeinsamkeit der befragten Fakultäten beschrieben. Einzelne Fakultätsleitungen beschrieben verschiedene Maßnahmen, die die Vorerfahrungen einzelner Lehrender für die gesamte Fakultät nutzbar machten. So wurden Good-Practice-Beispiele für andere als Orientierungshilfe nutzbar gemacht oder der Austausch unter den Lehrenden zur digitalen Lehrpraxis gefördert (Bosse, 2021). Alle Fakultätsleitungen stimmten darin überein, dass in Zukunft fächerübergreifend nicht auf die Präsenzlehre verzichtet werden könne, denn fachspezifische 
Praxisbezüge und Diskussionen seien digital nicht umsetzbar (Bosse, 2021). Die Gestaltung der Kontextbedingungen wurde gemeinsam mit Studierenden und Lehrenden umgesetzt. Dabei wurden zum Beispiel hochschulweite Abstimmungen genutzt, um die fakultätseigene digitale Lehre zu verbessern (Bosse, 2021). Diese Gemeinsamkeiten und Beispiele der Umsetzung an einzelnen Fakultäten wurden zusammengefasst, sodass sich daraus eine Liste von Lernmomenten ergab: Fakultäten profitierten von der zentralen wie der fakultätseigenen Infrastruktur, die didaktische Expertise Einzelner ließ sich hochschulweit und fakultätsspezifisch nutzen, die Präsenzlehre sei unabhängig von fachspezifischen Praxisbezügen und Rahmenvorgaben notwendig und fakultätsinterne Kommunikation war von großer Bedeutung für die digitale Lehre (Bosse, 2021). In der fallübergreifenden Ergebnisdarstellung wurden Gemeinsamkeiten der Fakultäten, deren Leitungen befragt wurden, herausgearbeitet. Gemeinsamkeiten waren, dass die digitale Lehre von gesamthochschulischen Infrastrukturen profitierte und eine eigene Ausstattung der Fakultät von Vorteil war. Einzelne Fakultäten nutzten die eigenen Informatik-Fachkompetenzen, um die digitale Lehre zu verbessern, schufen Didaktik-Stellen und warben Fördermittel ein (Bosse, 2021). Im Umgang mit der Krisensituation wurden die Vorerfahrung sowie der persönliche Einsatz der Lehrenden als Gemeinsamkeit der befragten Fakultäten beschrieben. Einzelne Fakultätsleitungen beschrieben verschiedene Maßnahmen, die die Vorerfahrungen einzelner Lehrender für die gesamte Fakultät nutzbar machten. So wurden Good-Practice-Beispiele für andere als Orientierungshilfe nutzbar gemacht oder der Austausch unter den Lehrenden zur digitalen Lehrpraxis gefördert (Bosse, 2021). Alle Fakultätsleitungen stimmten darin überein, dass in Zukunft fächerübergreifend nicht auf die Präsenzlehre verzichtet werden könne, denn fachspezifische Praxisbezüge und Diskussionen seien digital nicht umsetzbar (Bosse, 2021). Die Gestaltung der Kontextbedingungen wurde gemeinsam mit Studierenden und Lehrenden umgesetzt. Dabei wurden zum Beispiel hochschulweite Abstimmungen genutzt, um die fakultätseigene digitale Lehre zu verbessern (Bosse, 2021). Diese Gemeinsamkeiten und Beispiele der Umsetzung an einzelnen Fakultäten wurden zusammengefasst, sodass sich daraus eine Liste von Lernmomenten ergab: Fakultäten profitierten von der zentralen wie der fakultätseigenen In- 
frastruktur, die didaktische Expertise Einzelner ließ sich hochschulweit und fakultätsspezifisch nutzen, die Präsenzlehre sei unabhängig von fachspezifischen Praxisbezügen und Rahmenvorgaben notwendig und fakultätsinterne Kommunikation war von großer Bedeutung für die digitale Lehre (Bosse, 2021).

Auch Hochschulleitungen wurden qualitativ befragt. So wurden von Kreulich et al. (2020) Vizepräsident ${ }^{\star}$ innen bayrischer Hochschulen zu einer Expertendiskussion eingeladen. Ergebnisse waren, dass eine hochschulübergreifende sowie hochschulinterne Kooperation als wichtig empfunden wurde, dass eine veränderte Haltung der digital Lehrenden für die Vizepräsident*innen bemerkbar war, dass die flächendeckende Infrastruktur unter dem Gesichtspunkt der Rahmenbedingungen für digitale Lehre fehlte und dass Hochschulen einen Wandlungsprozess durchlaufen, der die Lehre bzw. das Studieren strukturell verändern wird. Dieser Wandlungsprozess sei nur in hochschulübergreifender Kooperation zu bewältigen.

\subsubsection{Internationale Studien}

In einer österreichischen Studie an der KPH Wien/Krems (Gabriel \& Pecher, 2020) wurden 35 Lehramtstudierende in narrativen Interviews nach ihrer Situation in den Corona-Semestern befragt. Hierbei wurde, wie Seyfeli et al. (2020) für Studierende in Deutschland bereits feststellten, die Flexibilität der digitalen Lehre von den österreichischen Studierenden als positiv genannt. Herausforderungen in den Corona-Semestern waren die fehlenden Instruktionen und Arbeitsanweisungen, die mangelnde Medienkompetenz der Lehrenden sowie die fehlende Einschätzung der Lehrenden bzgl. der an die Studierendensituation angepassten Größe der Arbeitsaufträge und der Art der Lehre. Hierzu gibt ein ${ }^{\star}$ e Studierende ${ }^{\star} r$ an, dass das teilweise mehrfache Lesen eines Textes deutlich aufwändiger wäre als es das direkte Frage-Antwort-Schema in den Veranstaltungen vor Ort war. Auch die Selbststrukturierung und die Organisation des Studienalltags wurden von den Studierenden als herausfordernd eingeschätzt sowie die fehlende nonverbale Kommunikation und physische Interaktion, die zu einem fehlenden Gemeinschaftsgefühl führten. Gespräche in den Veranstal- 
tungen seien intensiver, wenn sich Lehrende und Studierende physisch gegenübersitzen und sich anschauen könnten. Auch in dieser Studie ist die Gesamtbilanz ebenfalls ambivalent.

In einer weiteren Studie in Österreich von Krammer et al. (2020) wurden 75 Lehramtsstudierende mithilfe einer offenen schriftlichen Befragung nach ihrem subjektiv erlebten Lernerfolg gefragt. Für den Lernerfolg förderliche Aspekte waren Videokonferenzen, da hier Interaktion möglich war. Einzelarbeiten, die von anderen Studierenden unabhängiges Lernen ermöglichten, waren ebenfalls förderlich für den Lernerfolg. Die von den Lehrenden übersichtliche Aufbereitung und Strukturierung der Lernplattformen und das aktive Einbeziehen der Studierenden während der synchronen Veranstaltungen wurden ebenfalls positiv bewertet (Krammer et al., 2020). Hinderliche Aspekte für den eigenen Lernerfolg waren, so die von Krammer et al. (2020) befragten Studierenden, nicht nur unübersichtliche Informationen bzgl. der Veranstaltungen, sondern auch schlechte Internetverbindungen sowie fehlende Diskussionen. Auch hier ist eine ambivalente Gesamtbilanz zu sehen.

In einer ebenfalls österreichischen Studie (Vallaster \& Sageder, 2020) wurden 25 Studierende und acht Lehrende einer mittelgroßen Bildungsinstitution in Form von Tiefeninterviews befragt. Es wurde von den Lehrenden und Studierenden zusammenfassend gesagt, „dass die Akzeptanz digitaler Lerntechnologien im Verlauf der Krise eher abnahm“ (Vallaster \& Sageder, 2020, S. 294). Die Hochschule habe zukünftig die Aufgabe, durch Infrastruktur, Engagement und Know-how eine strategische Ausrichtung zu unterstützen. Hierbei würden auch die Anpassung der Curricula sowie klare Kommunikation helfen.

Auch wenn in diesem Rahmen nur einige Aspekte der Ergebnisse der Studien genannt werden konnten, lassen sich Chancen und Herausforderungen in den letzten beiden Corona-Semestern identifizieren, die für den Bereich der digitalen Lehre bereits bekannt und benannt sind (s. Kapitel 1). Dies sind zum Beispiel die hohe Herausforderung und der Kapazitätsaufwand sowie das mediendidaktische Geschick beim Aufbau von digitaler Lehre oder die Notwendigkeit der Organisation des eigenen Lernfortschritts aufseiten der Studierenden. Durch die bisher veröffentlichten Studien kann bereits ein breites Bild von der 
Situation an Hochschulen unter Corona-Bedingungen erlangt werden. Einige Aspekte sind jedoch bisher unbeleuchtet. In den hier beschriebenen Ergebnissen der Studien zur Situation von Studierenden, Lehrenden und Hochschulleitungen in den Corona-Semestern wurden bisher keine Good Practice-Fallbeispiele für digitale Lehre dargestellt. Die Lebenssituation außerhalb von Studium und Lehre unter den Corona-Maßnahmen und deren Auswirkungen auf die Lehr-/Lernsituation wurden in den bereits veröffentlichten Studien nur peripher behandelt. Darüber hinaus wurden die Akteur ${ }^{\star}$ innengruppen meist getrennt voneinander betrachtet, sodass keine Rückschlüsse auf das Zusammenspiel von Handlungs- und Lehr-/Lernbedingungen von Hochschulleitungen, Lehrenden und Studierenden gezogen werden konnten. Die vorliegende Studie greift explizit diese Themen auf. 


\section{Forschungsdesign: Forschungsfragen und Methode}

Wie in der Ausgangslage (s. Kapitel 1) ausgeführt, sind Konzepte zu digitaler Lehre seit Jahren vorhanden und sie werden im Hochschulsystem insbesondere im Kontext von Fernhochschulen und berufsbegleitenden weiterbildenden Studiengängen umgesetzt. Im Zusammenhang mit Herausforderungen und Potenzialen der Digitalisierung der Lehre war von einer neuen Verantwortungsrolle der Lehrenden und verschiedenen zu meisternden Dimensionen in der Umsetzung digitaler Lehre die Rede, angerissen wurde aber auch eine persönliche Haltung von Lehrenden als entscheidender Faktor für die Entwicklung hochwertiger digitaler Lehr-/Lernformate. Im Fokus stand ebenfalls der Aspekt zeitlicher und räumlicher Flexibilität digitaler Formate, der Hand in Hand mit der Frage nach mehr Eigenverantwortung und Selbstorganisation vonseiten der Studierenden und dem Bedarf einer Anpassung der Lehr-/ Lernstrukturen einhergeht. Eine weitere in der Problemlage beleuchtete Dimension bezog sich, unter Berücksichtigung neuer Interaktions- und Kommunikationswege, auf die Rahmenbedingungen, in denen Hochschule digital stattfindet, und nicht zuletzt wurde auf die Herausforderungen der pandemiebedingten Umstellung der Hochschullehre in ein digitales Format im Frühjahr 2020 eingegangen. Dass diese Ad-hocUmsetzung von Präsenz auf Digital auf verschiedenen Ebenen eine rundum neue Situation darstellte, zeigten auch mehrheitlich die Ergebnisse der Studien über die Auswirkungen der Corona-Pandemie auf die Hochschullandschaft, die in Kapitel 2 aus quantitativer und qualitativer Perspektive in den Blick genommen wurden. Ersichtlich wurde darin, dass die Hochschulen vor ganz neue Aufgaben und Herausforderungen gestellt wurden, zumal das bisherige Modell der Präsenzhochschule innerhalb von wenigen Wochen zum Modell einer geschlossenen digitalen Hochschule verändert werden musste und dies eine flächendeckende Umstellung erforderte. Zentrale Aspekte, die aus der Analyse der vorliegenden quantitativen Untersuchungen hervorgingen, umfassen zum einen die unzureichende technische Ausstattung von Hochschulen, 
Studierenden und Lehrenden, beziehen sich zum anderen auf die erhebliche Mehrbelastung, die die digitale Lehre für Lehrende sowie Studierende darstellte, sowie deren negative Auswirkungen auf das psychische und emotionale Befinden der Studierenden. Auch der fehlende Austausch vor Ort, der durch die digitale Lehre nicht kompensiert werden konnte, wurde den Studienergebnissen nach zu einer Herausforderung für alle Hochschulangehörigen. Grundsätzlich wurde dabei häufiger auf quantitative Untersuchungsmethodik zurückgegriffen. Die Ergebnisse der vorliegenden qualitativen Studien lassen erahnen, dass neben den Herausforderungen in der konkreten Lehr-/Lernsituation auch Lebensbereiche, die mit dem hochschulischen Leben assoziiert sind, vom Pandemiegeschehen stark betroffen sind und auch für Studium und Lehre wiederum eine erhöhte Relevanz haben. Eine vertiefende, ganzheitliche Untersuchung fand hier jedoch noch nicht statt. Eine Erhebung von Good-Practice-Fallbeispielen für digitale Lehre liegt bis dato ebenfalls hochschulübergreifend nicht vor. Vor dem Hintergrund der Ausgangslage setzt die Forschungsfrage hier an, die Auswirkungen der Corona-Pandemie auf die Hochschullandschaft und die Digitalisierung der Lehre aus verschiedenen Perspektiven in den Blick zu nehmen.

\subsection{Forschungsfragen und Aufbau der Studie}

Im Mittelpunkt der Studie steht die Forschungsfrage: Welche Entwicklungspfade für Hochschule und Lehre post Corona können aus den Erfahrungen der digitalen Semester abgeleitet werden? Der Fokus liegt dabei auf einer differenzierten Betrachtung der Situation an den HAWs in Baden-Württemberg in den beiden Corona-Semestern (SoSe 2020 und WiSe 2020/21) durch eine umfassende Nachzeichnung der Erfahrungen von Hochschulleitungen, Lehrenden und Studierenden. Die Untersuchung zielt auf empirisch gesicherte Ergebnisse ab, aus denen sich belastbare Erkenntnisse zur Entwicklung von Handlungsempfehlungen für Hochschulen post Corona hervorgehen. Vor dem Hintergrund dieser Zielsetzung setzte sich das Untersuchungsdesign inhaltlich-methodisch aus drei Zugängen zusammen, in denen jeweils unterschiedliche Teilforschungsfragen entwickelt wurden, um thema- 
tisch sowie zielgruppenspezifisch verschiedene Perspektiven zu erfassen. Abbildung 3 visualisiert die Forschungsschwerpunkte der drei Zugänge.

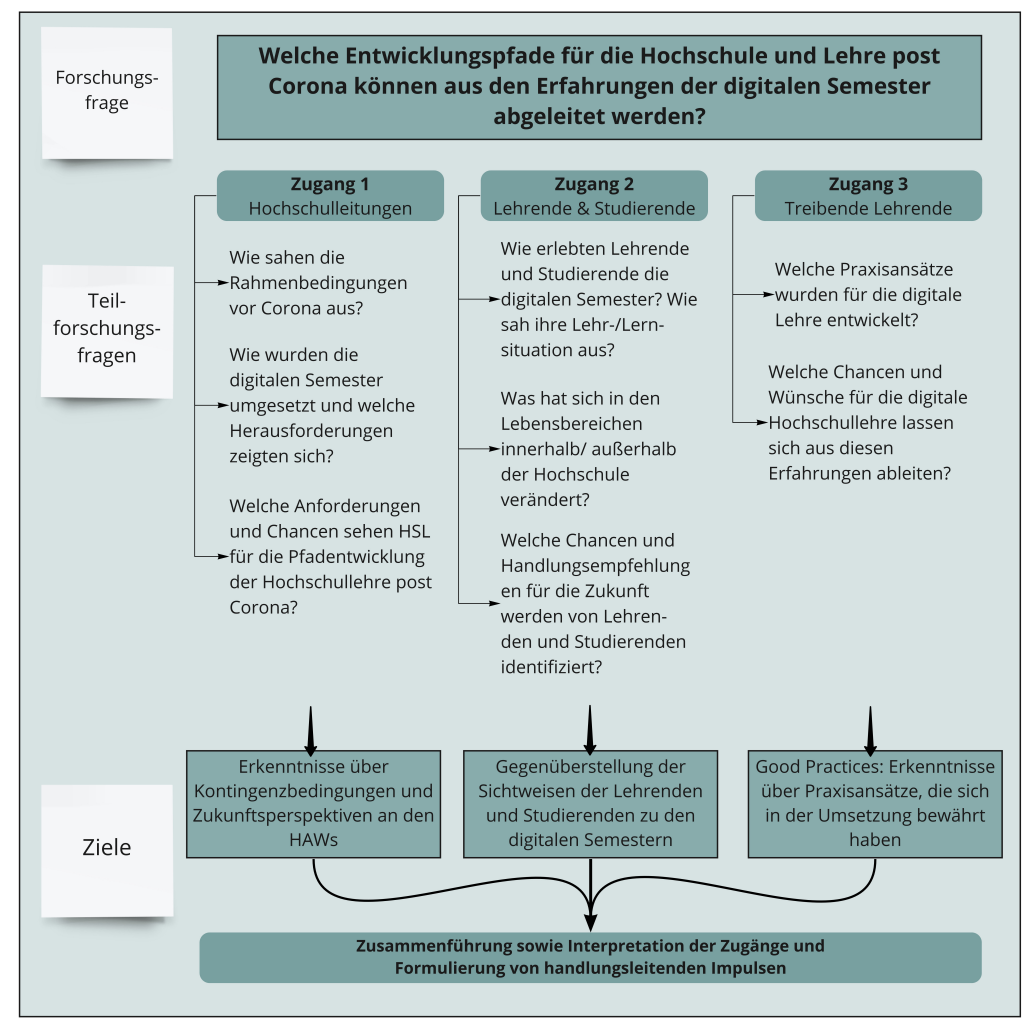

\section{Abbildung 3: Forschungsfragen der drei Zugänge}

Die Studie ist wie folgt aufgebaut: Im Zugang 1 (s. Kapitel 4) wird der Frage nach den Kontingenzbedingungen der Corona-Semester und der Zukunftsperspektive der Hochschulen post Corona aus Sicht der Hochschulleitungen nachgegangen. Der Kontingenzrahmen spannt sich im Spannungsfeld zwischen der absoluten Handlungsnotwendigkeit und der Unmöglichkeit der Situation und ihrer (teilweise nicht vorhandenen) Rahmenbedingungen auf. Hier entsteht ein Handlungsfeld, das es zu gestalten notwendig macht. Im Zugang 2 (s. Kapitel 5) wird untersucht, 
wie Lehrende und Studierende die Corona-Semester erlebt haben, vor welche Herausforderungen sie gestellt wurden und welche Chancen sie auf ihren bisherigen Erfahrungen basierend für die Hochschule post Corona sehen. Hier liegt das Augenmerk darauf, wie einzelne Personen und Personengruppen innerhalb der Kontingenzbedingungen agiert haben. Zugang 3 (s. Kapitel 6) widmet sich der Frage, welche Praxisansätze sich aus der Sicht von Lehrenden bewährt haben, die teils seit mehreren Jahren digitale Elemente in ihre Lehre einbinden und versuchen, an ihren Hochschulen die Digitalisierung voranzutreiben. Ziel ist hierbei, Good-Practice-Beispiele zu identifizieren und diese mittels Fallbeschreibungen zu erfassen. In Kapitel 7 werden die Ergebnisse der drei Forschungszugänge zusammengeführt und mit Bezugnahme auf die übergeordnete Forschungsfrage diskutiert und interpretiert. Darauf aufbauend werden Handlungsimpulse für Hochschule und Lehre post Corona entwickelt. Der Ausblick in Kapitel 8 resümiert die Studie.

\subsection{Methode}

\subsubsection{Qualitative Inhaltsanalyse}

Für die Beantwortung der forschungsleitenden Fragen wurde ein qualitatives Forschungsdesign gewählt. Ergänzend zu den bereits vorliegenden quantitativen Studien zu Auswirkungen der Corona-Pandemie auf das Studieren und Lehren an Hochschulen seit Sommersemester 2020 (s. Kapitel 2) wird in der vorliegenden Studie das Ziel verfolgt, die Perspektiven aller Beteiligten aus einer qualitativen Sicht zu beleuchten. Die Entscheidung für ein qualitatives Untersuchungsdesign ging also mit der Zielsetzung einher, die Beteiligten selbst zu Wort kommen zu lassen, um auf deren subjektives Erfahrungswissen zurückzugreifen, dabei differenzierte Bedeutungs- und Begründungszusammenhänge zu erfassen und darauf basierend ein möglichst umfassendes Bild der beiden CoronaSemester zu rekonstruieren. Durch ein vertiefendes Verstehen der Alltagserfahrungen besteht der Grundsatz des qualitativen Analyseverfahrens im Zuge der Mayringschen Tradition in der Einbettung des zu analysierenden Materials in größere Kommunikationszusammenhänge, 
um „latente Sinngehalte“ (Mayring \& Fenzl, 2014) in einem stark regelgeleiteten und intersubjektiv überprüfbaren Vorgehen zu erfassen.

Die empirische Datenerhebung basierte auf leitfadengestützten Einzelinterviews und Fokusgruppen (für eine Übersicht des Datenerhebungsverfahrens in den drei Forschungszugängen s. Abbildung 4 in Kapitel 3.2.2), die im Zeitraum von Januar bis März 2021 durchgeführt wurden. Für die Strukturierung der Befragung wurde in den drei Zugängen je ein Interviewleitfaden entwickelt, der sich thematisch an den jeweils zielgruppenspezifischen Teilforschungsfragen orientierte (genaue Erläuterung s. Kapitel 4, Kapitel 5 und Kapitel 6). Alle Interviews wurden mithilfe eines Videokonferenzsystems durchgeführt und nachträglich transkribiert. Die Transkription folgte den Regeln von (Kuckartz, 2018) und (Dresing \& Pehl, 2018). Zur weiteren computerunterstützten Auswertung in MAXQDA 2021 (VERBI Software, 2019) wurden die Interviews im Sinne einer zugesicherten Anonymisierung nach (Meyermann \& Porzelt, 2014) aufbereitet. Die Datenanalyse erfolgte auf der Grundlage von Kuckartz' inhaltlich-strukturierender Inhaltsanalyse, die Schreier treffend als eine Methode beschreibt, deren Kern darin besteht, „am Material ausgewählte inhaltliche Aspekte zu identifizieren, zu konzeptualisieren und das Material im Hinblick auf solche Aspekte systematisch zu beschreiben" (Schreier, 2014, S. 5). Ausgehend von den Teilforschungsfragen wurde in den Zugängen jeweils ein Kategoriensystem mit a priori definierten Hauptkategorien entwickelt, um die Interviews aussagekräftig strukturieren und möglichst viele Dimensionen abbilden zu können. Der inhaltlichen Datenauswertung folgten im letzten Schritt die Zusammenführung und die Interpretation der Ergebnisse aus den drei Zugängen vor dem Hintergrund der übergeordneten Forschungsfrage. Darauf aufbauend wurden Handlungsempfehlungen für Hochschule und Lehre post Corona konzipiert.

\subsubsection{Sampling}

Die Fallauswahl erfolgte in erster Linie auf der Basis inhaltlicher Repräsentativität und folgte dem Prinzip der Varianzmaximierung (Patton, 2002), d. h. die Interviews mit verschiedenen Personengruppen - sprich Mitgliedern von Hochschulleitungen, Lehrenden und Studierenden - 
zielten auf eine möglichst reichhaltige und vielfältige Einsicht in verschiedene Handlungsebenen der HAWs in Baden-Württemberg. Der Fokus lag dabei auf der Abbildung von Kontingenzbedingungen, Erfahrungen und Zukunftsperspektiven hinsichtlich digitaler Hochschullehre. An der Interviewstudie beteiligten sich elf von insgesamt 24 Hochschulen für Angewandte Wissenschaften in Baden-Württemberg, indem sie durch die Vermittlung von Interviewpartnerinnen den Feldzugang sicherstellten:

- Hochschule Aalen

- Hochschule Albstadt-Sigmaringen

- Hochschule Biberach

- Hochschule Esslingen

- Evangelische Hochschule Freiburg

- Hochschule Heilbronn

- Hochschule Karlsruhe - Technik und Wirtschaft

- Hochschule der Medien

- Hochschule für Wirtschaft und Umwelt Nürtingen-Geislingen

- Hochschule Reutlingen

- Hochschule für Gestaltung Schwäbisch Gmünd

Die Größe der beteiligten HAWs anhand der durchschnittlichen Studierendenzahl wurde in Abbildung 4 visualisiert.

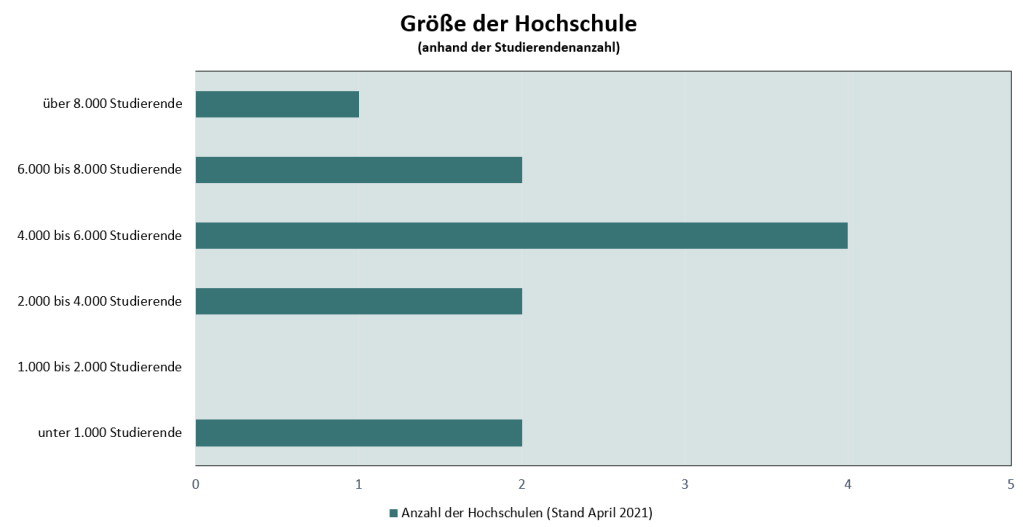

Abbildung 4: Größe der beteiligten HAWs (2021) 
Die Datengrundlage bestand aus 34 Interviews mit insgesamt 86 Teilnehmenden. Davon wurden im Forschungszugang 1 elf leitfadengestützte Einzelinterviews mit Hochschulleitungen geführt. Im Forschungszugang 2 fanden $15 \mathrm{HAW}$-übergreifende Fokusgruppen statt, jeweils in Gruppen von zwei bis sechs Professor*innen bzw. Bachelorstudierenden (darunter auch Lehrbeauftragte und Erst- bzw. Zweitsemester). Im Forschungszugang 3 wurden acht Einzelinterviews mit Lehrenden geführt, die durch ihre Vorerfahrung mit digitaler Lehre im Zuge der digitalen Umstellung der Lehr-/Lernsettings an ihren Hochschulen eine treibende Funktion innehatten. Die grafische Darstellung des Samples zeigt Abbildung 5.

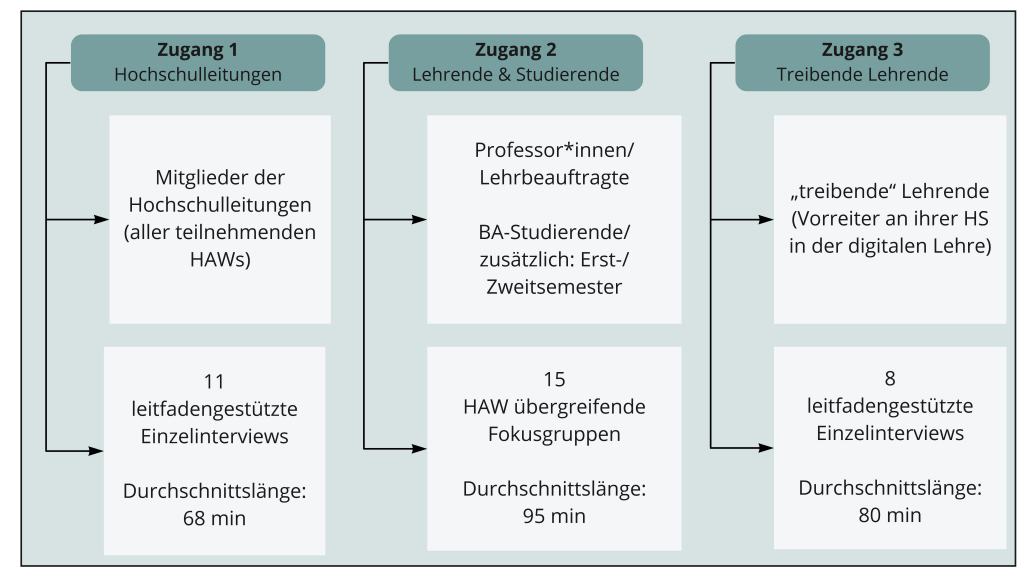

\section{Abbildung 5: Sampling in den drei Forschungszugängen}

In den folgenden Kapiteln werden die drei hier gezeigten Zugänge einzeln vorgestellt. Auf die detaillierte methodische Vorgehensweise in den drei Zugängen wird ebenfalls in den entsprechenden Kapiteln eingegangen. 


\section{Zugang 1: Kontingenzbedingungen und Zukunftsperspektiven aus Sicht der Hochschulleitungen}

Wie bereits in Kapitel 1 beschrieben, tat sich zu Beginn der Pandemie für die Hochschulen ein Möglichkeitsraum auf (s. Abbildung 2). Um einen Referenzrahmen zu schaffen, was innerhalb der Hochschulen und in den Lehr-/Lernsituationen während der Corona-Semester geschah, soll zunächst genauer untersucht werden, wie sich die Kontingenzbedingungen gestalteten. Um die Kontingenzbedingungen und Zukunftsperspektiven zu untersuchen, wurden Mitglieder der Hochschulleitungen befragt, da diese Akteur*innengruppe einen übergreifenden Einblick in die Bereiche der Hochschule und auch in die Rahmenbedingungen hat. Hochschulleitungen vertreten die Hochschulen sowohl nach außen als auch nach innen, indem z.B. strategische und operative Entscheidungen vorbereitet werden. Bisher wurden in nur wenigen Studien explizit Hochschulleitungen befragt (vgl. z.B. Bosse et al., 2020; Kreulich et al., 2020; s. Kapitel 2). Aus den genannten Gründen werden im vierten Kapitel Hochschulleitungen und ihre Perspektiven auf die Corona-Semester beleuchtet und somit in den Mittelpunkt gestellt.

\subsection{Untersuchungsgegenstand und methodische Vorgehensweise}

Im Forschungszugang 1 lag der Fokus auf der Erfassung der Kontingenzbedingungen, der Organisation der Corona-Semester sowie den Zukunftsperspektiven aus Sicht der Hochschulleitungen. Dazu wurden folgende Teilforschungsfragen formuliert (s. Abbildung 6). 


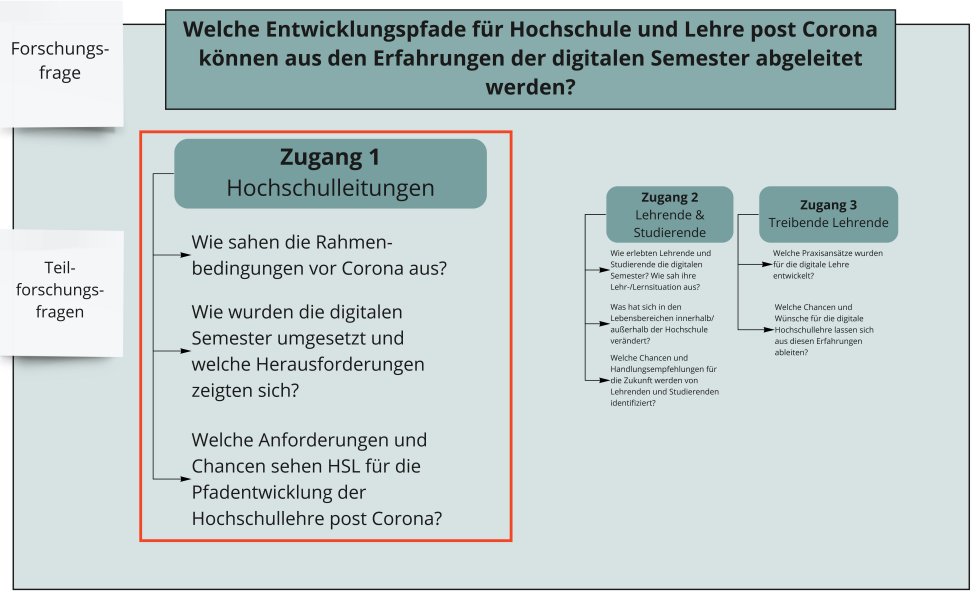

Abbildung 6: Teilforschungsfragen Zugang 1 im Gesamtzusammenhang

Hierfür wurden alle Hochschulleitungen der elf HAWs in BadenWürttemberg in Einzelinterviews befragt. Der dafür entwickelte Interviewleitfaden (s. Anhang 1) gliederte sich in fünf Abschnitte (s. Abbildung 7).

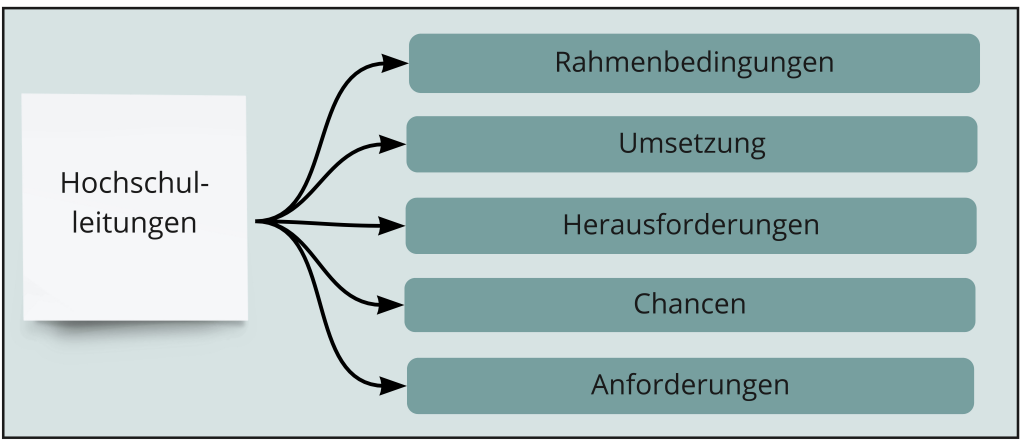

Abbildung 7: Leitfadenstruktur Zugang 1 
Im ersten Themenbereich wurden die allgemeinen Rahmenbedingungen und Voraussetzungen hinsichtlich des Digitalisierungsgrads im Februar 2020 fokussiert. Ferner wurde erfasst, welche Handlungen und Maßnahmen (Umsetzung) aufgrund der Corona-Pandemie durchgeführt wurden. Dazu wurden zum Beispiel Fragen zur Kommunikation, zu Entscheidungsprozessen und der Strategieveränderung der Hochschule gestellt. Der nächste Themenbereich zu den Herausforderungen diente dazu, Aussagen über die rückblickend größten Herausforderungen, die aktuellen Herausforderungen für Studierende, Lehrende, Verwaltung sowie die Hochschulleitungen selbst, zu erheben. Im anschließenden Themenbereich standen die Gefahren und Chancen der Corona-Pandemie für die Hochschulen im Fokus. Der letzte Teil des Leitfadens, der sich thematisch mit den Anforderungen und dem Ausblick beschäftigte, nahm die Perspektive auf die Zukunft der Hochschule post Corona in den Blick.

Hinsichtlich des methodischen Vorgehens wurden von Januar bis Februar 2021 elf leitfadengestützte Interviews (durchschnittliche Interviewdauer 68 Minuten) mit Hochschulleitungen (zwei Rektor ${ }^{\star}$ innen und neun Prorektor ${ }^{\star}$ innen im Bereich Lehre) geführt, auf Audio festgehalten und transkribiert. Die Auswertung der leitfadengestützten Interviews erfolgte mithilfe kategorienbasierter Verfahren in Orientierung an der qualitativen Inhaltsanalyse nach (Kuckartz, 2018), s. dazu Kapitel 3).

\subsection{Rahmenbedingungen}

Durch die Corona-Pandemie entstand im März 2020 ad hoc Druck von außen, welcher Handlungen in unsicheren Rahmenbedingungen notwendig machte. Die bisherigen Hochschulstrukturen mussten angepasst und neu organisiert werden, um den neuen Anforderungen im Hinblick der Lehr-/Lernformen, der Zusammenarbeit sowie der notwendigen technischen Ausstattung gerecht zu werden. Dabei kristallisierten sich verschiedene, zentrale Aspekte im Bereich der Rahmenbedingungen heraus (s. Abbildung 8). 


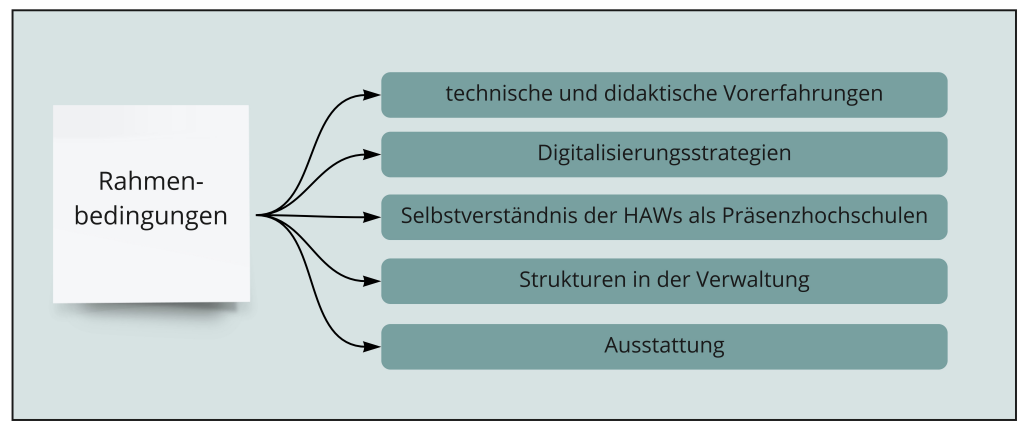

Abbildung 8: Zentrale Aspekte der Rahmenbedingungen

Der Großteil der interviewten Hochschulleitungen berichtete, dass sie im März 2020 auf didaktische und technische Vorerfahrungen und Vorarbeiten zurückgreifen konnten, die sich in der Umstellung auf den digitalen Lehrbetrieb als hilfreich erwiesen. Der Stand der Vorerfahrungen unterschied sich stark zwischen den Hochschulen. Einerseits konnte auf Erfahrungen von einzelnen Lehrenden zurückgegriffen werden, die bereits mit digitalen Lehrformaten arbeiteten. Somit waren bereits punktuell Elemente von digitaler Lehre im Einsatz. Diese Lehrenden übernahmen dadurch die Funktion von Vorreiter*innen und Treiber*innen bzw. dienten als Multiplikator*innen (für einen Einblick in die Erfahrungen mit digitalen Lehr-/Lernformaten dieser Lehrenden s. Kapitel 7). Eine der interviewten Hochschulleitungen fasste die Vorerfahrungen folgendermaßen zusammen: „Also die Lage war jetzt gar nicht so schlecht. Wir hatten schon als Hochschule einige Vorerfahrungen, natürlich nicht jeder Lehrende" (HSL2: 4). Auf der strukturellen Ebene wurde an manchen Hochschulen an die digitale Lerninfrastruktur und -formate der berufsbegleitenden weiterbildenden Studiengänge sowie an die Expertise der hochschulinternen Didaktikzentren angeknüpft. In den Hochschulen waren mehrheitlich Lernplattformen vorhanden, die Nutzung variierte jedoch in unterschiedlichem Ausmaß. In einer der interviewten Hochschulen wurde bereits 2019 hochschulweit die Lern- und Konferenzplattform ausgebaut. Zwei von elf Hochschulen konnten auf keine Vorarbeiten bzw. Vorerfahrungen in der digitalen Lehre zurückgreifen. 
In der Analyse des Interview-Materials zeigte sich, dass von einigen Hochschulen bereits eine Digitalisierungsstrategie ausgearbeitet worden war bzw. im Jahr 2020 entwickelt und/oder implementiert werden sollte. Daraus wurde deutlich, dass die Digitalisierung bereits vor der Corona-Pandemie ein strategisch relevantes Thema war. Vor Beginn der Pandemie gab es allerdings keinen akuten Handlungsdruck, die Digitalisierung in der Hochschullandschaft voranzutreiben. Zudem wurde in der Strategie der Hochschulleitungen der Schwerpunkt nicht auf die digitale Lehre, sondern auf die Digitalisierung der Verwaltungsprozesse gelegt. „Die Pandemie wirkt als Brandbeschleuniger für die Digitalisierung, aber ich glaube nicht, dass die Pandemie die Digitalisierung anders lenkt, als es mittelfristig ohnehin gekommen wäre" (HSL6: 40), so eine interviewte Hochschulleitung. Des Weiteren wurde in der Analyse deutlich, dass ein divergierendes Verständnis in Bezug auf den Begriff der Digitalisierung herrschte. Hochschulleitungen stellten sich die Frage, welche Maßnahmen zum heutigen Standard gehören und welche bereits unter Digitalisierung fallen. Das Beispiel einer der Hochschulleitungen verdeutlichte diese Problematik: „Und ich rede jetzt im Moment noch gar nicht mal von E-Learning, weil E-Learning ist schon noch mal für mein Empfinden noch mal was ganz andere an der Stelle. Also ich rede eigentlich nur noch mal von Digitalisierung vorhandener Unterlagen, die man einfach über diese Medien verwenden kann" (HSL8: 10).

Neben dem Aspekt der Digitalisierung wurde von den Hochschulleitungen mehrheitlich angegeben, dass sie die Hochschule als Präsenzhochschule verstehen. Aufgrund des Selbstverständnisses der HAWs als Präsenzhochschule gab es vordergründig keinen Druck zur Veränderung hin $\mathrm{zu}$ einer digitalen Lehre oder Fernhochschule. „Es ist schön, dass wir diesen Schub [in der Digitalisierung] jetzt haben, aber wir sind und bleiben eine Präsenzhochschule“, so eine der interviewten Hochschulleitungen (HSL10: 6).

Es zeigte sich außerdem, dass hinsichtlich der Rahmenbedingungen und Strukturen in der Verwaltung in den Interviews eine große Diskrepanz zwischen den hochschulinternen Verwaltungen herrschte. Im Detail konnte festgestellt werden, dass sich der Grad der Digitalisierung in den Verwaltungsstrukturen und -abläufen der Hochschulen stark unterscheidet. „Da ist schon ein Stück weit digitalisiert, aber 
nicht so, dass man es durchgehend quasi von der Bewerbung bis zum Abschlusszeugnis alles elektronisch hätte, sondern immer so Teilaspekte sind digitalisiert“, so eine der Hochschulleitungen (HSL4: 9). Es wurde in den Interviews formuliert: „Die Möglichkeiten der modernen Techniken, haben wir da eigentlich bei Weitem nicht ausgeschöpft“ (HSL7: 4). Bereits vor den Corona-Semestern wurde an der Digitalisierung von Verwaltungsprozessen in den studentischen Abteilungen wie z.B. Semesterrückmeldungen, digitale Prüfungsan- oder -abmeldungen ebenso wie Verwaltungsabläufe in den Haushalts- und Personalabteilungen gearbeitet. Laut den Interviews wurde die Digitalisierung dieser Prozesse durch den Ausbruch der Pandemie beschleunigt.

In der Analyse des Materials zeigte sich, dass der Themenkomplex der Ausstattung der Hochschulen und der Hochschulangehörigen eine wichtige Stellung innerhalb der Rahmenbedingungen einnahm. Aus Sicht der Hochschulleitungen waren die Lehrenden größtenteils mit Laptops ausgestattet. Die Studierenden verfügten mehrheitlich über eigene Endgeräte. Bisher bestand für sie die Möglichkeit der Nutzung der PC-Räume. Lediglich eine Hochschulleitung gab an, dass sie Studierende habe, die über keine eigenen Endgeräte verfügen und keinen Internetzugang haben. Bei den Mitarbeitenden in der Verwaltung war die technische Ausstattung weniger vorhanden, da bisher die Tätigkeiten mehrheitlich vor Ort erledigt wurden. Hier war der Stand an den Hochschulen ebenfalls uneinheitlich. So waren die Zugänge oder die Prozesse nicht in der benötigten Weise digitalisiert, dass die Tätigkeit im Homeoffice umgesetzt werden konnten. Videokonferenzsysteme waren zum Teil vorhanden, aber wurden noch nicht genutzt „Also wir hatten schon dieses Skype for Business, das hatten wir schon. Im Prinzip war jeder Rechner ausgerüstet. Mitnichten wusste jeder Mitarbeiter, dass das überhaupt auf seinem Computer ist" (HSL 2: 8), berichtete eine Hochschulleitung. In diesem Kontext tauchte hinsichtlich der Mitarbeitenden auch das Thema technische Regelungen bzw. Rahmenbedingungen für Homeoffice auf. Mehrheitlich war Homeoffice grundsätzlich möglich, zum Teil fehlten personalrechtliche Bestimmungen.

Zusammenfassend bestand eine enorme Diskrepanz hinsichtlich der Rahmenbedingungen an den befragten Hochschulen. Gemeinsam ist, dass an unterschiedliche Erfahrungen und Vorarbeiten angeknüpft wurde und dass das Selbstverständnis als Präsenzhochschule einen 
zentralen Aspekt für die Hochschulleitungen darstellte. Angesichts der Ad-hoc-Umstellung auf den digitalen Lehrbetrieb wurde eine große Anzahl an Neuerungen und Maßnahmen notwendig, die weit über das Vorhandene hinausgingen. Ausgehend von diesen Ergebnissen lassen sich wesentliche Erkenntnisse über Möglichkeiten und Notwendigkeiten in der Corona-Pandemie und damit Näherungen über die Spannbreite der Kontingenz ableiten.

\subsection{Umsetzung}

Aufbauend auf den Vorerfahrungen und den Grundvoraussetzungen (s. Kapitel 4.2), die im Speziellen mehr die pandemiebedingten Notwendigkeiten fokussierten, wurden erste Handlungswege skizziert, um die Möglichkeiten zu nutzen. Diese beziehen sich auf folgende wesentliche Aspekte (s. Abbildung 9).

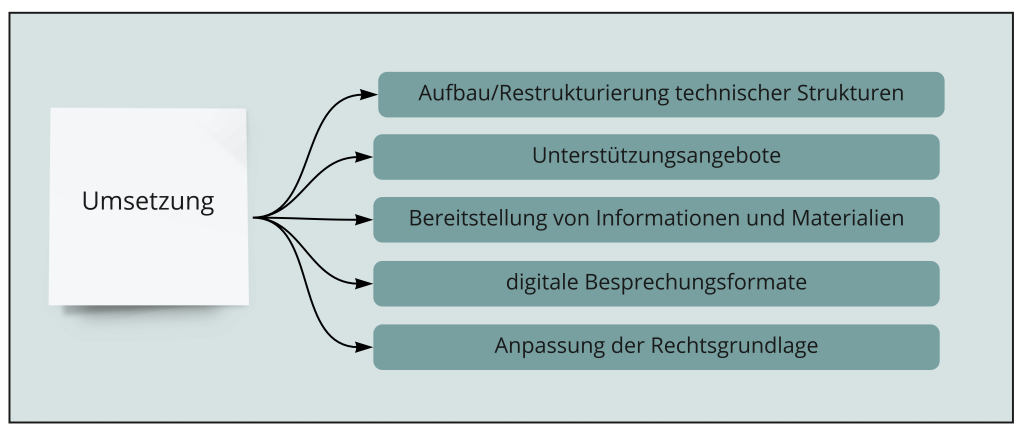

Abbildung 9: Zentrale Aspekte der Umsetzung

Aufgrund der Corona-Pandemie wurden Präsenzhochschulen sozusagen über Nacht zu geschlossenen digitalen Hochschulen. Um die neuen Anforderungen bewältigen zu können, wurden unterschiedliche Maßnahmen eingeleitet. Vor allem der Aufbau und die Restrukturierung der technischen Infrastrukturen und der Ausstattung waren zentrale Aufgaben zu Beginn des Pandemiegeschehens. Wie bereits beschrieben, waren die Hochschulen sehr unterschiedlich ausgestattet. Daher war der Bedarf an Aufbau und Reorganisation der technischen 
Infrastrukturen und der technischen Ausstattung enorm. Dazu wurde beispielsweise die IT-Zusammensetzung neu organisiert, um den aktuellen Bedürfnissen der Hochschulangehörigen $\mathrm{zu}$ entsprechen. Im Detail wurde, laut den Hochschulleitungen, in dieser Umbruchphase eine Educational IT zusammengeführt, da die Mitarbeitenden vor den Corona-Semestern auf verschiedene Organisationseinheiten verteilt waren. Dadurch wurden die Kompetenzen der IT-Mitarbeitenden gebündelt und diese übernahmen neben den Fakultäten einen Teil des Supports sowie die technische und didaktische Beratung. Die Analyse zeigte jedoch, dass dieses Vorgehen nicht von allen befragten Hochschulen in dieser Form umgesetzt wurde. Des Weiteren konnte in den Interviews festgestellt werden, dass Räume bzw. Hörsäle aufgerüstet (z.B. durch den Ausbau von LAN und WLAN) bzw. umgebaut wurden, um eine digitale Lehre zu ermöglichen. Eine Hochschulleitung erläuterte diesbezüglich, dass die Organisation der Auf- bzw. Umrüstung der Räume einen „Megaaufwand“ (HSL6: 12) bedeutete. Dazu wurden z.B. Sende- oder Empfangsräume gebaut, um Vorlesungen zu streamen. Ebenso wurde in den Interviews mit den Hochschulleitungen deutlich, dass im Hinblick auf die digitale Lehre Lizenzen von Programmen (vor allem Software für Videokonferenzsysteme) eingekauft bzw. aufgestockt wurden. Eine Hochschulleitung formulierte diesbezüglich: „Also das war dann schon auch ein finanzieller Akt, so viele ZoomLizenzen anzuschaffen“ (HSL11: 12). Dieses Zitat verdeutlicht, dass Hochschulen neben der pandemiebedingten Umstellung und dem damit verbundenen organisatorischen Aufwand, hinsichtlich ihrer finanziellen Mittel an Grenzen kamen.

Neben dem Aufbau und der Restrukturierung wurde aus der Analyse deutlich, dass die Initiierung von Unterstützungsangeboten für die Hochschulangehörigen ein weiterer zentraler Aspekt war. Dafür wurde z.B. Hardware (Headsets, Laptops mit spezieller Software etc.) für Studierende bzw. allgemein Hochschulangehörige eingekauft. Diese Hardware wurde dann je nach Bedarf über das Rechenzentrum ausgeliehen, damit bestenfalls alle Hochschulangehörigen von zu Hause arbeitsfähig waren. Laut den befragten Hochschulleitungen wurde der Aufbau einer qualifizierten Supportstruktur für Lehrende und Studierende zu einer zentralen Aufgabe. Dafür wurden die zuständigen Supportstrukturen (z.B. Ansprech-Erste-Hilfe für Studierende) mit zu- 
sätzlichen Stellen versehen oder über Tutor*innen ausgebaut. Für Fragen oder Probleme wurden bis zu vier Mal die Woche Sprechstunden mit Tutor ${ }^{*}$ innen (auch E-Tutor*innen genannt) eingerichtet, in denen sich Studierende und Lehrende Unterstützung holen konnten. „Und diese E-Tutoren, das hat sich eigentlich bewährt, die kommen sehr, sehr gut an. Und damit haben wir eigentlich relativ viel abgedeckt, was so die Betreuung und die Fragen anging" (HSL3: 18), sagte eine der Hochschulleitungen dazu. Die Unterstützungsangebote für die Hochschulangehörigen beinhalteten außerdem die Durchführung von Schulungen. Den Interviews zufolge war es den Hochschulleitungen wichtig, "die Lehrenden, aber auch die Studierenden in der Anwendung dieser Infrastruktur [und der Programme] zu schulen“ (HSL1: 4). Diese Schulungen zielten insbesondere darauf ab, Berührungsängste der Lehrenden sowie der Studierenden mit digitalen Werkzeugen bzw. generell digitaler Lehre abzubauen.

Da bei den Lehrenden, wie oben beschrieben, punktuell Erfahrungen mit digitaler Lehre vorhanden waren, wurden Einführungskurse für unterschiedliche Themen angeboten (z.B. Anmeldung in Programmen und Möglichkeiten und Grenzen der Programme). Die Lehrenden wurden dabei insbesondere von wissenschaftlichen Hilfskräften in der digitalen Lehre unterstützt. Beispielsweise hatten Lehrende die Möglichkeit, das digitale Lehren mit wissenschaftlichen Hilfskräften auszuprobieren, einerseits um Berührungsängste zu reduzieren, und andererseits, um neue Formate zu testen. Bei Bedarf wurden die Lehrenden beispielsweise vom hochschulinternen Didaktik-Zentrum unterstützt, indem sie die Möglichkeit hatten, an mehreren Schulungsveranstaltungen bzw. Informationsveranstaltungen (in synchroner als auch in asynchroner Form) teilzunehmen. In diesen Veranstaltungen wurden didaktisches Know-how, Hilfeformate sowie Tipps und Tricks, wie digitale Lehre gestaltet werden kann, bereitgestellt.

Die Studierenden wurden vonseiten der Hochschule einerseits unterstützt, indem Leihgeräte (z.B. Headsets oder Kameras) und Lernräume zur Verfügung gestellt und eine Hotline für Fragen eingerichtet wurde. Die Hochschulleitungen verdeutlichten in den Interviews die Relevanz, Studierende und vor allem Erstsemester in die Hochschule und ihre Strukturen einzuführen. Dafür wurde ein interaktives Begleitprogramm für Studierende entwickelt, welches verschiedene Un- 
terstützungsmaßnahmen (insbesondere für Erstsemester) beinhaltet. Darin wurden Tipps rund um die Themen Selbstorganisation und -motivation, digitales Lernen sowie Prüfungsängste gegeben. Es wurden von den Hochschulen zudem virtuelle Campustage und Sonderveranstaltungen für Erstsemester initiiert, da es laut den Hochschulleitungen schwierig war, an die Erstsemester heranzukommen. Aus diesem Grund „war unser Hauptaugenmerk, die Erstsemester, dass man die auch ein bisschen gesondert behandelt" (HSL4: 30). Es zeigte sich in der Analyse des Materials, dass die Durchführung von Maßnahmen in den Verwaltungen der Hochschulen zu Beginn der Corona-Pandemie als nachrangig wahrgenommen wurde. „Wir haben in der zweiten Welle, nachdem wir die Lehrenden versorgt hatten ... dann auch die Verwaltung mitgenommen" (HSL1: 6).

Neben den Unterstützungsangeboten für die Hochschulangehörigen zeigte sich in der Auswertung das Thema der Bereitstellung von Informationen und Materialien. Generell wurden Informationen und Materialien zu verschiedensten Themen für die Hochschulangehörigen durch unterschiedliche Medien (E-Mail, Plattformen, Webseite etc.) bereitgestellt. Beispielsweise wurden die neuesten Verordnungen auf neu entstandenen hochschulinternen Corona-Webseiten hochgeladen oder Best-Practice-Beispiele, Lessons Learned, Lehrvideos und vieles mehr zur Verfügung gestellt. Studierende erhielten auf diesen Wegen auch die neuesten Informationen über die anstehenden Prüfungen. Außerdem wurden Handreichungen und Handouts von Hochschulleitungen selbst, den hochschulinternen Didaktik-Zentren oder neu entstandenen Teams (auch Corona-Taskforce genannt) verfasst.

Wir haben dann recht schnell ein Team zusammengestellt, was sich eben darum gekümmert hat auszuloten: Was für digitale Medien brauchen wir an der Hochschule? Was für digitale Unterstützungsmaßnahmen brauchen wir an der Hochschule? Und wo ist der Unterstützungsbedarf sowohl auf Seite der Lehrenden als auch auf Seite der Studierenden? (HSL7: 10)

Die Corona-Taskforces der Hochschulen übernahmen neben dem Verfassen von Handreichungen und Einführung von Programmen (z.B. Wie funktioniert Zoom/Teams?) weitere Aufgaben, wie das Überprüfen von Alternativprogrammen, Durchführen von Schulungen und die Klärung zentraler Begrifflichkeiten (z.B. Was wird unter synchroner 
oder asynchroner Lehre verstanden). Zum Zeitpunkt der Befragung bestanden die Corona-Taskforces nach wie vor. Es zeigte sich jedoch, dass der Bedarf nach diesem intensiven Austausch und dem Austesten nicht mehr notwendig ist, da ein gewisser „Normalbetrieb“ (HSL2: 18) eingekehrt ist.

Während der Analyse zeigte sich, dass ein zentraler Fokus aus Sicht der Hochschulleitungen auf der Umstellung und Einführung digitaler Besprechungsformate lag. Einerseits wurde diese Entwicklung relevant, damit das Treffen von Entscheidungen wie bisher möglich und Arbeitsgruppen weiterhin arbeitsfähig waren. Es wurde von den Hochschulleitungen beschrieben, dass lange Besprechungen vor Ort kein Problem darstellten, jedoch in digitaler Form. Dadurch, dass wenig Austausch und inhaltliche Diskussionen im Rahmen der digitalen Besprechungen stattfanden, bestand eine Notwendigkeit, neue Formate für Besprechungen zu entwickeln. Laut einer Hochschulleitung ist der Austausch in Besprechungen „eine andere Art von Diskussionskultur“ (HSL4: 36), da vor allem die Gestik und Mimik bei ausgeschalteter Kamera fehlen oder schlechter wahrzunehmen sind. Andererseits wurden neue Besprechungsformate benötigt, um informellen Austausch zwischen Hochschulangehörigen digital zu ermöglichen, da sich der Großteil der Hochschulangehörigen im Homeoffice befand und der persönliche Austausch am Arbeitsplatz dadurch wegfiel. Aus diesem Grund wurden beispielsweise Brown-Bag-Lunches, E-Cafés oder Didaktik-Treffs angeboten, während derer einzelne, für die Lehrenden interessante Themen, besprochen und Erfahrungen ausgetauscht wurden. Eine Hochschulleitung beschrieb diesbezüglich:

Was sich dann selbst initiiert hat, war in vielen Fakultäten zunächst mal auch ein kollegialer Austausch, also, so wie man sich früher in Nicht-Corona-Zeiten am Kaffeeautomaten getroffen hat, um miteinander zu sprechen, haben sich manche Fakultäten zunächst mal eben zu einem Onlinekaffee verabredet und konnten sich dann da auch einmal oder zweimal miteinander austauschen und so einen Best-Practice- oder einen Good-Practice-Austausch machen. (HSL1: 16)

Des Weiteren wurde von Dekan^innen teilweise die Frequenz der Sitzungen erhöht oder Sitzungszeiten wurden verlängert, um insbesondere neuen oder weniger vernetzten Mitarbeitenden Kommunikationsmöglichkeiten und -räume zu ermöglichen. Dies wurde außerdem vor 
dem Hintergrund eingeführt, dass Dekan*innen durch den Wegfall der informellen Gespräche einen Nachteil in der Wahrnehmung ihrer Führungsaufgaben sahen.

Als Folge der Umstellung auf einen digitalen Hochschulbetrieb wurde aus den Interviews deutlich, dass Hochschulleitungen auch vor der Aufgabe der Schaffung angepasster rechtlicher Rahmenbedingungen standen. Maßnahmen im Bereich der rechtlichen Rahmenbedingungen betrafen vor allem die Klärung datenschutzrechtlicher Fragen und die allgemeine Gewährleistung der Rechtssicherheit von digitalen Lösungen für die Lehre und Prüfungen, aber auch für Verwaltungsvorgänge und die Gremienarbeit. Dafür verfassten die Hochschulleitungen eigene Datenschutzsatzungen in Zusammenarbeit mit den hochschulinternen Datenschutzbeauftragten, um „einigermaßen rechtskonform" (HSL11: 12) zu arbeiten. Hierbei wurde erwähnt, dass die HAWs eng zusammenarbeitete, sich austauschte und unterstützte. Dem Bedarf an rechtlichen Neuregelungen wurde in Form von Corona-Satzungen bzw. Verordnungen entsprochen, welche einige Hochschulen aufsetzten und kontinuierlich anpassten. Andere Hochschulen setzten wiederum keine Corona-Satzung auf, sondern integrierten die neuen Aspekte der digitalen Lehre in das bestehende System und in die Studienprüfungsordnung. "Also es ist auch nicht beabsichtigt, die wieder rauszunehmen, sondern wir haben das versucht gleich als Chance zu sehen und zu sagen: Wir nehmen das. Das wird nicht mehr verschwinden“, so eine der Hochschulleitungen (HSL2: 30). In einer hochschulinternen Evaluation wurde laut einer Hochschulleitung sehr deutlich, dass zwei Drittel der Hochschulangehörigen dafür plädieren, digitale Lehranteile im Curriculum zu verankern. In der Analyse zeigte sich außerdem, dass sich die Hochschulleitungen mit dem Thema der Deputatsabrechnung auseinandersetzen mussten. Hochschulleitungen stellten sich diesbezüglich die Frage, wie das Deputat abgerechnet wird, wenn Videos einmal hergestellt und in Zukunft lediglich hochgeladen werden. Diese hochschulrechtlichen Fragen wurden intensiv behandelt und führten $\mathrm{zu}$ einem erhöhten Abstimmungsbedarf mit den Fakultäten und Dekanaten. Dazu fehlten jedoch genaue Vorgaben seitens des Ministeriums. Des Weiteren wurden von den Hochschulleitungen Geschäftsordnungen geändert, damit Beschlüsse online gefasst werden durften. Die Herstellung von Rechtssicherheit, resümierte eine 
der Hochschulleitungen, wurde zu einer sehr großen Aufgabe. Dazu wurde beschrieben: „Wir machen es anständig. Wir machen es, glaube ich, auch gut. Aber wir können es natürlich nichts rechtskonform hinkriegen. Also sobald Corona auch nur im Ansatz vorbei ist, fliegt uns das hier alles um die Ohren" (HSL11: 40). Neben den rechtlichen Rahmenbedingungen wurden spezifische Regelungsbedarfe im Bereich der Studien- und Prüfungsorganisation von den Hochschulleitungen genannt. $\mathrm{Zu}$ den genannten Maßnahmen gehörten nicht nur Änderungen von Studien-, Prüfungs- und Immatrikulationsordnungen und die Anpassung von Fristen und Vorgaben, sondern auch Hinweise auf die Entwicklung neuer Prüfungsformate, die Nutzung neuer Prüfungssoftware und die Erstellung von Handreichungen für das Lehrund Prüfungsmanagement. Für die Studierenden wurden zudem die Regelungen für Urlaubs- und Praxissemester angepasst. Die befragten Hochschulleitungen wiesen darauf hin, dass Urlaubssemester vermehrt in Anspruch genommen wurden, um die Corona-Semester zu überbrücken.

Zusammenfassend zeigte sich, dass die Hochschulleitungen eine enorme Bandbreite an Aufgaben zu bewältigen hatten, um den Hochschulangehörigen eine digitale Lehre sowie Weiterarbeit zu ermöglichen. Diese Aufgaben unter Pandemiebedingungen zu organisieren, stellte die Hochschulleitungen in manchen Fällen vor große Herausforderungen. Es zeigt aber auch, welche Möglichkeiten Hochschulen gefunden haben, um im Rahmen des Kontingenzraums auf die Notwendigkeiten und Anforderungen zu reagieren.

\subsection{Herausforderungen}

Die Hochschule insgesamt wurde im Pandemiegeschehen vor eine Vielzahl von Herausforderungen gestellt, die im Kapitel 4.2 und Kapitel 4.3 bereits angeklungen sind, in diesem Abschnitt aber noch konkretisiert und systematisiert werden sollen. Folgende Aspekte sind dabei wesentlich (s. Abbildung 10). 


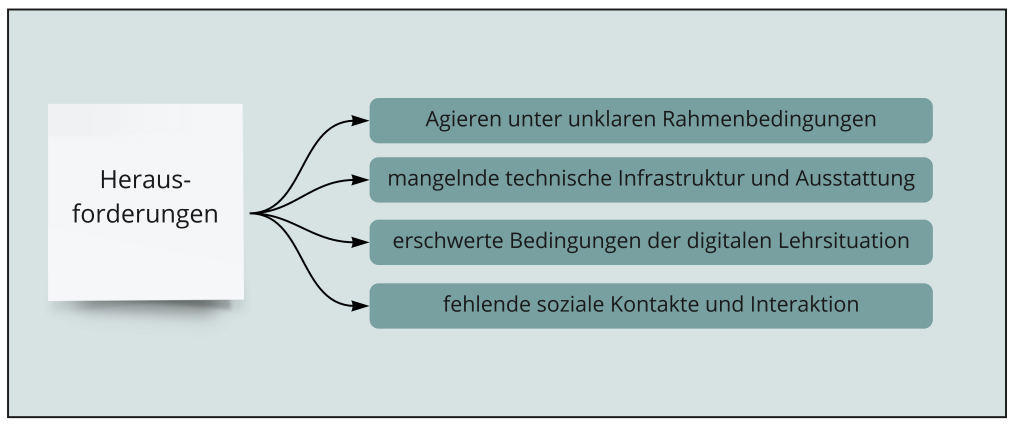

Abbildung 10: Zentrale Aspekte der Herausforderungen

Die Hochschulleitungen sahen sich mit der Notwendigkeit konfrontiert, für die Hochschule und somit für alle Mitarbeitenden, Lehrenden und Studierenden Entscheidungen zu treffen. Eine vorausschauende Planung war angesichts der unvorhersehbaren Entwicklung der Corona-Pandemie - auch nach über einem Jahr - nahezu unmöglich. Die Hochschulen standen unter akutem Handlungsdruck, sie „konnten nicht warten, bis die letzte Verordnung fertig war, das war einfach nicht drin" (HSL8: 24). In der Analyse konnte festgestellt werden, dass das Agieren und Reagieren unter unklaren Rahmenbedingungen für die Hochschulleitungen eine Belastung darstellten, da nicht eingeschätzt werden konnte, worauf sich die HAWs (kurz- oder mittelfristig) einstellen mussten. Eine der größten Herausforderungen war „das Umstellen von Präsenzhochschule auf Fernhochschule, das war ein extremer Wechsel“ (HSL4: 70). Die Ad-hoc-Umstellung führte dazu, dass es zu Beginn des ersten Corona-Semesters keine Rechtsgrundlage gab. Die Rechtsgrundlage wurde erst nachträglich im Landeshochschulgesetz (LHG) angepasst. Es zeigte sich außerdem in der Analyse, dass die schnelle Taktung und Anpassung und das schnelle Reagieren-Müssen Druck auf die Hochschulleitungen ausübte: „Ja, natürlich, man schläft ja schon unruhig in diesen Zeiten, weil man natürlich immer mit sich ringt, sind die Entscheidungen, die man trifft, die richtigen, ja? Ich meine, das lässt uns ja nicht kalt, ja?" (HSL10: 34). Eine Hochschulleitung sprach von einem „Spagat“ zwischen dem Anbieten von Präsenzprüfungen und der enormen Verantwortung des Schutzes 
der Hochschulangehörigen, da bei Präsenzprüfungen „viele Leute auf engem Raum zusammen [sitzen] in einer Zeit, wo ein Corona-Virus mit Mutationen wütet" (HSL10: 34). Von den Hochschulen wurden aufgrund dessen Hygienekonzepte für Präsenzprüfungen entwickelt, jedoch bestand keine Planungssicherheit, da es zu keiner Zeit absehbar war, wie sich die Inzidenzen entwickeln. „Unter Bedingungen der Unsicherheit Entscheidungen zu treffen und auch $\mathrm{zu}$ vertreten gegenüber Kollegen und Kolleginnen“ (HSL9: 34), wurde von den Hochschulleitungen des Weiteren als belastend genannt. Die Analyse zeigte zu diesem Aspekt, dass Hochschulleitungen es als Herausforderung empfanden, die unterschiedlichen Vorstellungen und Bedarfe der Hochschulangehörigen zu berücksichtigen. Es wurde deutlich, dass die verschiedenen Akteur ${ }^{\star}$ innengruppen divergente Ansichten hatten, wie die Hochschule mit der Corona-Pandemie umgehen sollte. „Also das zu moderieren, das bringt mich dann doch manchmal auch an meine Grenzen, so als Dompteur. Also es ist wirklich schwierig“ (HSL11: 40). Es wurde seitens der Hochschulleitungen versucht, die unterschiedlichen Bedarfe „einzusammeln und in eine Form von Ausgleich zu bringen“ (HSL9: 34). Insbesondere wurde hier die Relevanz der internen Kommunikation genannt, indem die Hochschulleitungen formulierten, dass die Bedarfe wahrgenommen und unter deren Berücksichtigung Entscheidungen getroffen wurden. Die größte Problematik zeigte sich insbesondere darin, dass die Hochschulen Entscheidungen selbstständig trafen (bzw. treffen mussten) mit der Befürchtung, dass das „Ministerium noch irgendwas [sagt], was das Ganze für unrechtmäßig erklärt" (HSL9: 34). Diese Unsicherheit wurde dadurch erschwert, dass teilweise unklare bzw. zu späte Informationen aus den Ministerien bereitgestellt wurden. In den Interviews wurde dazu stark verdeutlicht, dass aufgrund dessen die Auseinandersetzung mit dem Ministerium „wirklich Nerven gekostet hat" (HSL9: 34)

Das Ministerium [ist] auch in der Situation, dass sie ja auch kurzfristig rechtliche Vorgaben erhält. So. Und diese kurzfristigen rechtlichen Vorgaben wurden eben ähnlich kurzfristig dann an uns weitergereicht oder sagen wir mal noch etwas kurzfristiger dann an uns weitergereicht. Das ist für uns in der Hochschulleitung herausfordernd gewesen in der Umsetzung und ist es auch jetzt noch. (HSL7: 36) 
Die Auswertung zeigte, dass die Hochschulleitungen Verständnis für die Schwierigkeiten in der Kommunikation mit dem Ministerium hatten, da alle Beteiligten kurzfristig auf das Pandemiegeschehen reagieren mussten. Diese Verzögerung in der Kommunikation hatte jedoch Auswirkungen auf Prozesse innerhalb der Hochschulen. „Die Studierenden wenden sich an uns und beschweren sich, warum wir keine klareren Richtlinien rausgeben. Aber wir können es nicht, weil wir ja auch entsprechend kurzfristig die Vorgaben erhalten" (HSL7: 36). Diese Frustration über nicht vorhandene Informationen war insbesondere für die Studierenden und Lehrenden groß, da sie „am Montagmorgen um acht mit den neuen Regularien umgehen müssen“ (HSL1: 30). In den Interviews wurde deutlich, dass die kurzfristige Kommunikation von Änderungen der rechtlichen Rahmenbedingungen zu Widerständen bei den Hochschulangehörigen führte.

Ein weiterer Themenschwerpunkt, der in den Interviews prominent wurde, beinhaltet die mangelnde technische Infrastruktur und Ausstattung. Aufgrund der Ad-hoc-Umstellung waren die Hochschulen, insbesondere die IT-Ausstattung sowie das Rechenzentrum, nicht darauf vorbereitet. Die Herausforderungen der technischen Infrastruktur und Ausstattung lagen z.T. an mangelndem Netzausbau bzw. der Instabilität des Internets, wie auch an der notwendigen Schaffung der technischen Voraussetzungen aufseiten der Lehrenden.

Problem war, dass halt, ich sage mal von der Infrastruktur her, Rechner, Handys natürlich nicht darauf vorbereitet waren, sodass das am Anfang dann doch schwieriger war, ich würde jetzt sagen, dass wir momentan wirklich am Anfang die Leute dann auch teilweise hilflos waren und auf eigene Geräte zurückgreifen mussten, weil sie eben dann auch kein Laptop daheim hatten. (HSL10: 18)

Zugleich standen Hochschulleitungen vor dem Problem, dass eingekaufte Programme nicht funktionierten und Alternativen gefunden werden mussten. Nach dem Einkauf der Programme formulierte eine Hochschulleitung im Interview: „Und jetzt sitzen wir hier vor digitalen Werkzeugen und haben überhaupt keine Erfahrungen" (HSL11: 38). Die mangelnden Vorerfahrungen und die damit einhergehenden Berührungsängste der Lehrenden stellten laut der Analyse des Materials eine enorme Herausforderung dar. Der Abbau dieser Berührungsängste und der Aufbau von Medienkompetenz wurden somit zentra- 
le Aufgaben, um digitale Lehre überhaupt zu ermöglichen. Damit einher ging ebenso die Bereitschaft der Lehrenden, entsprechendes Know-how aufzubauen, neue Dinge auszuprobieren und tatsächlich in die Lehre zu integrieren. Auf der Seite der Studierenden gab es diesbezüglich laut den Hochschulleitungen weniger Probleme. Es gab Einzelfälle, in denen die Infrastruktur nicht ausreichend war oder wo technische Voraussetzungen, wie eine Webcam, fehlte. Diese Probleme wurden durch die Unterstützungsmaßnahmen der Hochschulen (z.B. Zurverfügungstellung von Räumen oder Leihgeräten) gelöst.

Die erschwerten Bedingungen der digitalen Lehrsituation wurden aus Sicht der Hochschulleitungen als Herausforderungen identifiziert. Durch die Corona-Pandemie und die damit einhergehende digitale Lehre veränderte sich vor allem die hochschulinterne Kommunikation. "Ich meine, dieses ganze Zwischenmenschliche geht verloren" (HSL10: 14), es wird „sehr viel unpersönlicher" (HSL4: 36) und schwieriger, "alle mitzunehmen und zu begeistern für das Fach" (HSL6: 32), so die Hochschulleitungen. Die Übertragung von Emotionen, Gestik und Mimik ist in der digitalen Lehre bedeutend schwieriger als in der Präsenzlehre. Dieser Aspekt wird noch dadurch intensiviert, dass Studierende die Kamera während des Seminars bzw. der Vorlesung nicht anschalten. „Eigentlich redet man gegen einen schwarzen Vorhang, gefühlt. Es kommt wenig zurück“ (HSL6: 32) und „Ich selbst fand das auch frustrierend, dann die [schwarzen] Kacheln zu sehen" (HSL10: 6), formulierten die Hochschulleitungen und wiesen darauf hin, dass Kolleg*innen Ähnliches berichteten. Somit ist die Ablenkungsgefahr bei den Studierenden größer, da niemand sehen kann, mit was sich die Studierenden wirklich beschäftigten.

Also man hat sich gefühlt wie ein Radio, das irgendwo im Hintergrund plärrt und die Studenten machen da was weiß ich was. Manche machen dann auch Fehler, schalten aus Versehen ihr Mikro ein und dann kriegt man mit, dass die gerade in der Küche sind, sich was zu essen machen. Ein anderer Fall ist, wo zwei Studierende sich unterhalten haben über ihren Hund und das ist zufällig eben angeschaltet. Da kommt man sich schon ein bisschen blöd vor. (HSL4: 40)

Des Weiteren ist aus Sicht der Hochschulleitungen der Erwerb von sozialen Kompetenzen im digitalen Format problematisch. Ein Studium ist mehr "als Wissensaufnahme“ (HSL6: 34). Die Vermittlung von 
Fachwissen ist digital in guter Qualität möglich, aber Kompetenzen, die über das Fachwissen hinausgehen (z.B. Teamarbeit), sind digital schwieriger zu vermitteln. Laut den interviewten Hochschulleitungen fehlt Studierenden der Austausch, und die digitale Lehre deckt diese Bedarfe und Bedürfnisse nicht ab. Vor allem den höheren Semestern fehlen die fachlichen Diskussionen. Um mehr Austausch und Zusammenarbeit in der digitalen Lehre zu ermöglichen, müssen passende digitale Formate entwickelt bzw. gefunden werden, aber vor dieser Herausforderung stehen die Hochschulen noch. Dabei gab es insbesondere bei HAWs die Problematik, dass manche Studiengänge eine Anwesenheit vor Ort voraussetzen. Einige Inhalte des Studiums können nicht digitalisiert werden, wie z.B. die Arbeit im Labor, an Maschinen oder im Fernseh- und Tonstudio.

Aus der Perspektive der Hochschulleitungen stellten die fehlenden sozialen Kontakte und die fehlende Interaktion durch die digitalen Lernsettings die größten Herausforderungen für die Studierenden dar. Die digitale Lehrsituation ist für alle Studierenden, insbesondere für Erstsemester, herausfordernd, da sich die Studierenden untereinander noch nicht kennen. Den interviewten Hochschulleitungen zufolge sind auch Studierende z.B. aufgrund finanzieller Aspekte (Wegfall vieler Nebenjobs) zurück zu den Eltern gezogen. Somit hatten die Studierenden lediglich über die digitale Lehre Kontakt zu Kommilitonen. „Und dieses Dranbleiben, gerade wenn es dann viele Abgaben sind, und dann muss man sich zwingen, irgendwie nicht nur den ganzen Tag vor dem Bildschirm zu sitzen“" (HSL3: 46). Die Studierenden meldeten den Hochschulleitungen zurück, dass sie diese Art des Studierens als „extrem stressig“ (HSL3: 46) empfanden. Die Hochschulleitungen sahen dort die Gefahr der Vereinsamung und Isolation und aufgrund dieser Tatsache ein erhöhtes Risiko, dass Studierende die Hochschule verlassen und somit das Studium abbrechen. Die Anforderungen an Selbststeuerung und -regulierung, Selbstkontrolle im Studium und Motivation sowie der erhöhte Arbeitsaufwand waren für einige Studierende eine Belastung und Überforderung. Dazu formulierte eine der Hochschulleitungen aus Sicht eines Studierenden Folgendes: „Es fällt mir schwerer, mich zu strukturieren, es fällt mir schwerer mich $\mathrm{zu}$ motivieren. Ich bin unsicher, wo mein Leistungsstand ist und ja. Ich habe eigentlich den Eindruck, ich lerne weniger als vorher" 
(HSL5: 28). Somit ergibt sich aus Sicht der Hochschulleitungen eine erhöhte Arbeitsbelastung der Studierenden. Die ohnehin vorhandene Leistungsstreuung unter diesen hat sich durch das Homestudying weiter erhöht. Die Studierenden, die in den Corona-Semestern größere Schwierigkeiten in der Selbstorganisation hatten, sind weiter zurückgefallen, wohingegen Studierende, die sich besser organisieren konnten, die Herausforderungen des Corona-Semesters kompensieren bzw. ihre Leistungen verbessern konnten. „Wie gesagt, es kommt eine Gruppe zurecht, es kommt eine Gruppe weniger zurecht mit ganz unterschiedlichen Auswirkungen" (HSL5: 38), so eine der Hochschulleitungen.

Zusammenfassend wurde festgestellt, dass die Hochschulleitungen und die Hochschulangehörigen durch das Pandemiegeschehen vor einer großen Varianz an Herausforderungen standen und diese zum Großteil mit erheblichem Mehraufwand bewältigen konnten. Dies spiegeln sich auch in den Vorerfahrungen und der Umsetzung wider. Die Hochschulleitungen reflektierten in den Interviews den Prozess und ihre Erfahrungen vom Beginn der Pandemie bis zum Zeitpunkt der Befragung und formulierten daraus Chancen für die zukünftige Hochschullandschaft.

\subsection{Chancen}

Die Hochschulleitungen sahen viele Chancen durch die Erfahrungen der Corona-Semester sowohl auf der strukturellen, der didaktischen als auch auf der strategischen Ebene zu profitieren. Hier eröffnen sich viele Spielräume in der Kontingenz unter folgenden Aspekten (s. Abbildung 11). 


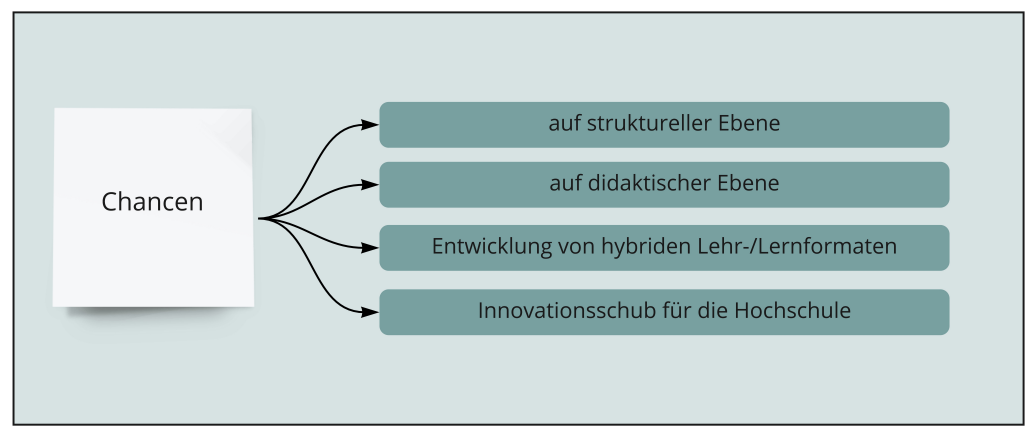

Abbildung 11: Zentrale Aspekte der Chancen

Auf der strukturellen Ebene zeigten sich neue flexible Gestaltungsmöglichkeiten. Studierende mit besonderer Lebenssituationen (z.B. gesundheitliche Beeinträchtigungen, Berufstätigkeit, die Pflege eines Familienmitglieds oder einer angehörigen Person, Betreuung eines Kindes) könnten je nach Format von zu Hause an Veranstaltungen teilnehmen, diese von unterwegs verfolgen oder im Nachhinein anhören. „Das sehe ich als Chance, auch vielleicht für Studierende, die eben zeitlich, räumlich gewisse Einschränkungen haben aus unterschiedlichen Gründen - familiär, gesundheitlich“ (HSL6: 36). Einerseits wären digitale Veranstaltungen für Studierende im Praxissemester ein enormer Vorteil und andererseits wären semesterübergreifende Veranstaltungen einfacher planbar und durchführbar. Bei Besprechungen und Lehrveranstaltungen könnten externe Teilnehmende, z.B. Fachexpert $^{*}$ innen oder Kooperationspartner*innen, problemlos von einem anderen Ort an einer Besprechung teilnehmen. Zudem könnten Lehrbeauftragte leichter akquiriert werden, wenn ein Teil der Semesterwochenstunden digital unterrichtet und sich somit Anfahrtswege reduzieren würden. Des Weiteren sah ein Teil der Hochschulleitungen Chancen für die Weiterentwicklung bestehender Studiengänge sowie den Ausbau des Weiterbildungsangebots. Durch den Digitalisierungsschub sind Lehrende bedeutend vertrauter im Umgang mit digitaler Lehre, wodurch neue digitale Weiterbildungsangebote konzipiert werden könnten Eine Hochschulleitung beschrieb dazu, dass es bereits digitale Weiterbildungsangebote gab, aber die Chance wird darin gese- 
hen, Kooperationen mit internationalen Studiengängen bzw. Partnerhochschulen einzugehen. Wie erläutert, lassen sich gemeinsame Projekte oder Lehrveranstaltungen mit internationalen Partnern ${ }^{*}$ innen bzw. Partnerhochschulen realisieren, ohne dass Wissenschaftler*innen oder Studierende zwingend ins Ausland gehen müssten. Dabei geht es den Hochschulleitungen vor allem darum, dass die „Internationalisierung zu Hause vorangetrieben“ (HSL10: 52) wird und die Möglichkeit für Studierende besteht „auf unserem Campus internationaler [zu] studieren" (HSL10: 52). Eine der interviewten Hochschulleitungen formulierte bezugnehmend $\mathrm{zu}$ dieser Chance: „Ich habe hier einen tollen Kurs an Hochschule X absolviert online, war voll super, und ich studiere aber an Hochschule Y, wo ich mir das dann entsprechend anerkennen lasse" (HSL1: 44). Diese Niedrigschwelligkeit, die durch den Einsatz digitaler Werkzeuge bzw. Lehre erzeugt wurde, bezieht sich nicht nur auf die Internationalisierung, sondern ebenso auf die Kommunikation. Es wurde von den Hochschulleitungen festgestellt, dass sich die bisherige Struktur bzw. der Rahmen durch die Digitalisierung verändert hat und dadurch gewisse Hürden und Hierarchiestufen verschwinden. Daraus wurde abgeleitet, dass sich die Umgangsformen sowie der Sprachgebrauch verändert haben: Jemanden „anzuchatten“ wird leichter, denn die Kommunikation findet bedeutend mehr auf Augenhöhe statt.

Auf der didaktischen Ebene sahen die Hochschulleitungen Chancen für einen Diskurs. „Eigentlich zwingen uns Corona und die Digitalisierung gerade dazu, jede einzelne Lehrveranstaltung noch mal auf ein neues Konzept umzustellen und kritisch zu hinterfragen und eben nicht ein ich mache das weiter so" (HSL1: 48). Das Zitat verdeutlicht, dass der Ausbruch der Corona-Pandemie zu einer Überarbeitung der Lehre führte. Im Kontext des Lebenslangen Lernens veränderte sich laut den Hochschulleitungen die Rolle der Hochschule und der Lehrenden. „Den Studierenden wirklich helfen, weiter voranzukommen“ (HSL6: 40) und nicht jedes Semester in gleicher Weise zu gestalten, das ist der Anspruch. Wissen ist heutzutage schnell abrufbar und die Hochschulen müssten eher die Rolle des Wissensnavigators übernehmen und die Lehrenden die Funktion des Lernbegleiters bzw. des Coachs. Dadurch sind Lehrende "nicht mehr in der Rolle [dessen], der was vorliest, sondern der seine Studierenden coacht“ (HSL2: 44). 
Die Schwierigkeit, die Studierenden individuell zu unterstützen, liegt darin, dass die Unterstützungsbedarfe unterschiedlich sind. Durch den Einsatz digitaler Formate ist eine individuelle Lernunterstützung durch die Lehrenden eher umsetzbar. Durch Videoaufzeichnungen und digitale Selbstlerntools könnten unterschiedliche Lerntempi und flexibleres Lernen ermöglicht werden. Die Problematik der Lehrenden besteht darin, dass der individuelle Leistungsstand ihrer Studierenden dadurch unbekannt ist. Aus Sicht der Hochschulleitungen könnten Rückmeldungen über den Leistungsstand durch Selbsttests oder Umfragen eingeholt werden, damit Studierende leichter einschätzen könnten, auf welchem Leistungsstand sie sich befinden und wo ggf. noch Lernbedarf ist.

Insgesamt sahen die Hochschulleitungen in der Entwicklung von hybriden Lehr-/Lernformaten post Corona eine Chance hinsichtlich der Hochschulentwicklung. Auf einen „breiteren Strauß an ... Lehrveranstaltungen und natürlich auch einen breiteren Strauß an Prüfungen, Prüfungsformen“ (HSL10: 52) könnte aufgebaut werden. Wie dieses Modell aussehen könnte, wurde von den Hochschulleitungen unterschiedlich eingeschätzt. Einigkeit bestand jedoch darin, dass die Vorteile digitaler Elemente genutzt und mit der Präsenzhochschule verbunden werden sollten. Dazu sagte eine Hochschulleitung im Interview: „Wo es sinnvoll ist, es digital zu ergänzen oder digital durchzuführen, da wollen wir es digital machen. Das heißt, wir gehen davon aus, dass wir so eine Art Mischhochschule, hybride Hochschule, wie auch immer man das nennen will“ (HSL3: 60), werden. Laut den befragten Hochschulleitungen müsste im nächsten Schritt geprüft werden, welche Elemente digital umsetzbar wären und welche Elemente vor Ort durchgeführt werden müssten, um daraus einen systematischen Ansatz zu entwickeln, wann digitale Formate sinnvoll sind und wann nicht.

Wir haben jetzt ganz viel entwickelt. Es sind da ganz viele Erfahrungen mit der Digitalisierung der Lehre, und die Fragestellung, die jetzt ansteht, ist, im Prinzip die Spreu vom Weizen zu trennen und wirklich eine kriteriengeleitete Diskussion zu haben: Was eignet sich für Digitalisierung und was wollen wir auch nicht digitalisieren, wenn wir jetzt nach Corona schauen. (HSL5: 44) 
Dafür bedarf es entsprechender Kriterien, die zu erarbeiten sind. Die auf der Leitungsebene bereits länger diskutierten oder sich in Umsetzung befindenden Digitalisierungsstrategien erhielten durch die Corona-Situation entsprechende Impulse.

Daran anknüpfend sahen die Hochschulleitungen insgesamt auf der strategischen Ebene einen Innovationsschub für die Institution Hochschule als eine große Chance, denn vor allem zu Beginn des Pandemiegeschehens wurden immense Vorbehalte gegen eine digitale Lehre geäußert.

Okay, ich begreife das wirklich gleich als Chance. Wann hätte ich jemals all meine Lehrenden dazu bekommen, freiwillig sich mit dem Thema auseinanderzusetzen? Das ist ja nicht so, dass wir das Thema nicht vorher auch schon versucht haben in die Breite auszurollen, aber da sagte halt jeder: Ja, das läuft doch und ist eigentlich. Also, man hat ja immer eine Ausrede gehabt. Insofern hatte ich jetzt von außen den Druck, dass sich wirklich alle damit auseinandersetzen mussten, und insofern, glaube ich, haben wir uns da schon enorm auch nach vorne katapultiert, wenn auch gegen den eigenen Wunsch, ja. Also wir haben es immer auch als Chance gesehen. (HSL10: 6)

Hemmschwellen wurden abgebaut und es gab ein gemeinsames Ausprobieren und Anwenden von den Hochschulangehörigen. Die Hochschulen haben „einen riesengroßen Push gekriegt, den es ohne Corona in der kurzen Zeit mit Sicherheit nicht gegeben hätte" (HSL7: 34). Die bereits vor Corona begonnene Digitalisierung von Prozessen könnte durch den Schub des Pandemiegeschehens nun vehementer weitergeführt werden. Darin liegen Chance und Anforderung zugleich. Beispielsweise formulierte eine der befragten Hochschulleitungen: „Aber der Wunsch ist ganz viel, zum Beispiel papierlos zu arbeiten. Das ist bei uns noch nicht der Fall“ (HSL9: 50). Prozesse könnten dadurch vereinheitlicht und die Bearbeitungsschritte effizienter erfolgen. „Also die Chance ist natürlich: Wir haben jetzt alle angefangen, alles Mögliche $\mathrm{zu}$ digitalisieren, nicht nur die Lehre, sondern auch die ganzen Strukturen drumherum. Das war bei uns mehr als nötig" (HSL11: 42). Während der Umstellung auf die digitale Lehre wurden Lehrinhalte überarbeitet und in digitalen Formaten neu aufgesetzt. Hier fungierten einige Lehrende als Treiber und diese hatten gewisse „Vorbildfunktionen" (HSL1: 6), wobei sie weitere Lehrende motivierten, sich mit der digitalen Lehre auseinanderzusetzen (auf die Funktion und die 
Rolle der treibenden Lehrenden wird in Kapitel 6 näher eingegangen). Und dieser Schwung sollte laut den Interviews mit den Hochschulleitungen aufgenommen werden, um sich als Hochschule „innovativ“ aufzustellen und evtl. eine „Vorreiterrolle“ (HSL3: 50) einzunehmen, da sich die Einstellung zur Digitalisierung bzw. digitalen Lehre radikal verändert hat. Aufgrund des Digitalisierungsschubs zeigte sich in der Erweiterung der digitalen Kompetenzen der Studierenden hinsichtlich des Umgangs mit digitalen Formaten für ihre zukünftigen Arbeitstätigkeiten ein weiterer Vorteil. In den Interviews bezogen sich die Hochschulleitungen auf den Aspekt, dass die Arbeitswelt, ebenso wie die Hochschulen, digitaler würde und somit andere Kompetenzanforderungen an die Studierenden gestellt werden. Aus diesem Grund sahen Hochschulleitungen die Chance dieses Schubs, um Studierende auf die zukünftige Arbeitswelt vorzubereiten.

Wie von mehreren Hochschulleitungen beschrieben, entstand ein neues Bewusstsein. Didaktische Diskurse wurden durch die Umstellung auf die digitale Lehre wieder geführt und sollen, so wünschen es sich die Hochschulleitungen, weiter vorangebracht werden, da sich eine neuartige Offenheit für Veränderungen zeigte.

Und ich glaube wirklich, mit unserem Senat vor zwei Jahren hätte man wahrscheinlich große Schwierigkeiten gehabt damit, dazu solche Beschlüsse zur weitergehenden Digitalisierung herbeizuführen. Aber dadurch, dass jetzt jeder eben damit umgehen muss und merkt, dass es auch geht. Dadurch ist, glaube ich, da ganz, ganz viel Offenheit gewachsen, ja. (HSL7: 72)

Entscheidungen konnten schneller beschlossen werden, die sonst bürokratischer und langwieriger waren. Es ist wie der Beginn eines Change-Prozesses gewesen mit der Herausforderung, alle Hochschulangehörigen mitzunehmen und niemanden zu verlieren. „Dann wird es Hochschulen geben, die fallen sehr schnell wieder zurück ins alte Fahrwasser. Und dann wird es Hochschulen geben, die werden durch die Erfahrungen jetzt im letzten Jahr, aber auch in $2021 \mathrm{mit}$ diesen Erfahrungen sich weiterentwickeln" (HSL1: 58). Dazu wurde die Befürchtung geäußert, ob dieser begonnene Change-Prozess weitergeführt werden könne oder ob die Gefahr bestehe, zurück in alte Prozesse oder Doppelstrukturen zu fallen sowie Neuerungen nicht dauerhaft etablieren zu können. 
Die beschriebenen Chancen auf der strukturellen, didaktischen und strategischen Ebene zeigten, dass durch die Corona-Pandemie die Flexibilisierung der Zusammenarbeits- und Lernkultur erreicht wurde und Prozesse beschleunigt wurden. Daraus ergeben sich Anforderungen, die zukünftig von den Hochschulen bearbeitet werden müssen, um die - seit Beginn der Corona-Pandemie - umgesetzten Maßnahmen, die bewältigten Herausforderungen und daraus resultierenden Chancen langfristig zu implementieren und die Hochschullandschaft nachhaltig zu verändern.

\subsection{Anforderungen}

Für die Pfadentwicklung der Hochschulen sahen die Hochschulleitungen Anforderungen hinsichtlich der Hochschulstrukturen, der politischen Rahmenbedingungen sowie des Selbstverständnisses der HAWs. Folgende Aspekte sind dabei wesentlich (s. Abbildung 12).

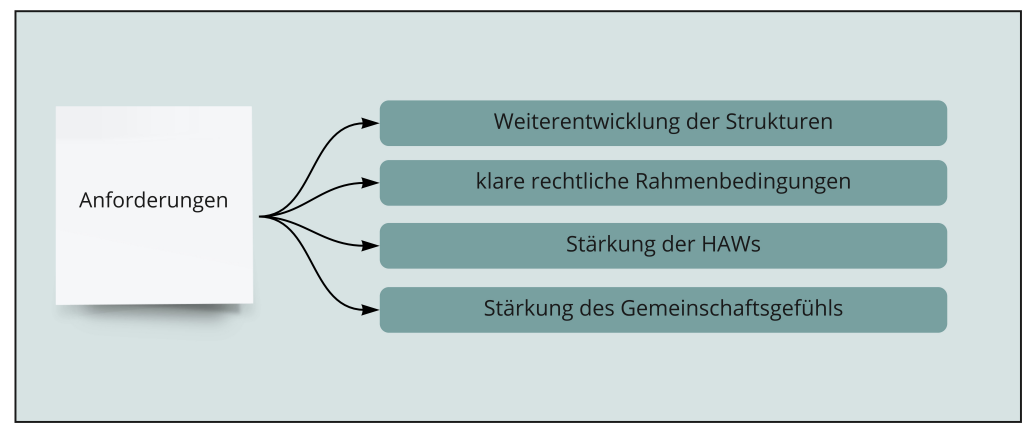

Abbildung 12: Zentrale Aspekte der Anforderungen

Die Hochschulleitungen nannten als zukünftige Anforderungen die Weiterentwicklung und Anpassung von Hochschulstrukturen. Die Integration digitaler Lehrformate sowie die Digitalisierung der Verwaltungsprozesse müsste weiter beschleunigt und umgesetzt werden. Prozesse müssten nun angepasst und überarbeitet werden, da sie aufgrund des Handlungsdrucks erst mal kurzfristig verändert wurden. In 
diesem Kontext müssten Lösungen gefunden werden, damit sowohl Rechts- als auch Datensicherheit bei der Nutzung von Videokonferenzund Datenmanagementsystemen gewährleistet ist. Damit einher ging die Anforderung, die notwendige technische und didaktische Ausstattung für hybride Lehr- und Lernmöglichkeiten weiter auszubauen. Ohne eine gesicherte Finanzierung war die nachhaltige Implementierung der begonnenen Maßnahmen nicht möglich. „Wir brauchen eine dauerhafte, eine gescheite Finanzierung, damit wir auch die Sachen mal angehen können. Mit diesen dauerhaften temporär finanzierten Sachen, das ist ein Problem“ (HSL4: 84). Für eine wirklich gute digitale Lehre erforderte es Unterstützung aus dem IT-Bereich, um die Konzepte didaktisch weiterzuentwickeln, wie in den Herausforderungen (s. Kapitel 4.4) bereits ausführlich beschrieben wurde. In der Analyse des der Transkripte zeigte sich, dass die Anforderung nach didaktischer und technischer Ausstattung mit Anforderungen an die Infrastruktur auf dem Campus verbunden war. Die gesamte Raumsituation müsste überdacht werden, inwiefern die heutigen Lehrräume und Hörsäle mit fester Bestuhlung in Ausrichtung zur Tafel noch zu den zukünftigen Lehr-/Lernformaten passen und welche Änderungen hier notwendig werden.

Und insofern, denke ich, ein bisschen andere Lernformen, auch Lehrräume, weg von diesen Vorlesungshörsälen, die reduzieren, dafür attraktive Kleinst- und Kollaborationsräume schaffen für Studierende. Das, das wäre so die so eine Wunschvorstellung von mir. Ich glaube, das wäre eine attraktive Mischung für Studierende. (HSL2: 52)

Neue Anforderungen ergaben sich aus Sicht der Hochschulleitungen an die Personalführung. Durch die Prozessveränderungen wandeln sich auch Aufgabenbereiche der Hochschulangehörigen. „Das hat auch Konsequenzen für das Thema Führen und Leiten, auch wenn ein Teil der Leute teilweise immer zu Hause ist" (HSL5: 56). Dazu gehörte zum einen die Schaffung der strukturellen Voraussetzungen zum mobilen Arbeiten durch eine entsprechende Personalvereinbarung, zum anderen die Sicherstellung der digitalen Informationsflüsse und der entsprechenden Infrastruktur. Dabei müsste auch die Gruppe der Lehrbeauftragten im Blick behalten werden - insbesondere hinsichtlich der Vergütung von digitaler Lehre sollte die Situation verbessert werden, erläuterte eine Hochschulleitung. Eine weitere Anforderung sah die 
Hochschulleitung in der Zusammenführung der verschiedenen Bedarfe und Vorstellungen der Hochschulangehörigen. Durch die Flexibilisierung der Lehr-/Lern- und Arbeitssituation entstanden seitens der Studierenden, Lehrenden wie auch Mitarbeitenden unterschiedliche Bedarfe und Ansprüche.

Also ich glaube, es ist auch eine Erwartungshaltung unter Studierenden gewachsen, sodass zum Beispiel Vorlesungsaufzeichnungen bereitgestellt werden, dass man eben flexibler ist darin, Lehrinhalte abzurufen. (HSL7: 54)

Die Hochschulleitungen müssten mit den Beteiligten entsprechende Lösungen entwickeln, um die Hochschulstrukturen für die neuen Erfordernisse auszurichten. Durch die Corona-Pandemie sind viele neue Möglichkeitsräume entstanden und die Aufgaben der Hochschulleitungen liegt nun darin, daraus entsprechende Richtungsentscheidungen herbeizuführen.

Die Hochschulleitungen forderten klare rechtliche Rahmenbedingungen, um die Möglichkeitsräume zu gestalten, die durch die Pandemie entstanden sind. Einige Hochschulleitungen gingen auf das Spannungsfeld zwischen Freiheit und Flexibilität einerseits und Regulierung und Vereinheitlichung andererseits ein. „Also ich denke, da ist so ein Riesenhandlungsfeld eigentlich, wo man sich mehr Unterstützung, mehr, ja, mehr Freiheit, vielleicht auch mehr Flexibilität und einfach etwas, ja, einen weiteren Spielraum wünschen könnte" (HSL3: 54). Hier wurde deutlich, dass sich Hochschulen auch selbst für die Gestaltung des Möglichkeitsraums verantwortlich fühlten und dies nicht nur den Landesorganen überlassen wollten, aber dafür auch entsprechenden Rahmen benötigen. Für die Hochschulleitungen bestand ein enormer Handlungsdruck, kurzfristig hochschulinterne Entscheidungen zu treffen. Sie hatten nach bestem Wissen und Gewissen als Hochschulverantwortliche entschieden und zusätzlich an anderen Stellen Unterstützung und Vernetzung gesucht. „Jetzt a posteriori Regeln festzulegen, nach denen wir uns dann messen lassen müssen, rückwirkend, das fände ich komplett verfehlt" (HSL10: 48). Eine Hochschulleitung äußerte diesbezüglich ihre Bedenken, dass dadurch ein generelles Misstrauen gegenüber allen Lehrenden mitschwingt, dass im Rahmen der digitalen Lehre weniger gearbeitet wurde. „Ich würde mir wünschen, das Ministerium würde uns mal fragen, was wir uns 
eigentlich vorstellen, was uns guttäte, was erhöht die Qualität von Lehre, Forschung und Weiterbildung im Blick auf Digitalisierung in den Hochschulen?" (HSL9: 54). Die Corona-Pandemie ist eine noch nie da gewesene Ausnahmesituation, auf die ad hoc reagiert werden musste. Der Aufwand, um die digitale Lehre zu ermöglichen, war immens.

Und ich sage mal so: Wenn wir nicht so eine super gut funktionierende Geschäftsstelle hätten der HAW BW dann, glaube ich, wäre auch für viele Hochschulleitungen im Land das Ganze viel konfliktreicher gewesen. (HSL9: 34)

Den Hochschulleitungen war durchaus bewusst, dass das Ministerium ebenso unter den gegebenen Unsicherheiten agieren musste, dennoch erschwerten die fehlenden Regelungen die rechtliche Umsetzung der digitalen Lehre, sodass jede Hochschule ein eigenes Modell entwickeln musste. Die langfristige strukturelle Weiterentwicklung der Hochschulen post Corona erfordert nun ein Zurechtrücken von den bereits mehrfach beschriebenen Themen von z.B. Datenschutz und -sicherheit, Lehrdeputatsabrechnungen, Onlineprüfungen. Die Hochschulen benötigen Gewissheit, über wie viel Handlungsspielraum sie verfügen und welche Grenzen seitens des Ministeriums gesetzt werden. Sie benötigen seitens des Ministeriums eine klare und transparente Kommunikation, um Prozesse entsprechend anpassen zu können, da bisherige Vorgaben und Regelungen nicht mehr passen. Dazu gehörten auch Antworten bezüglich einer dauerhaften Finanzierung der technischen und digitalen Ausstattung.

Es gibt viele rechtliche Themen, die noch nicht hinreichend geklärt sind, auch Deputatsabrechnungen usw. Und das sind tatsächlich Fragestellungen, die eine einzelne Hochschule nicht klären kann, sondern wo aus meiner Sicht eine übergeordnete Klärung erforderlich ist, mindestens durch einen HAW-Verband, eigentlich eher sogar noch deutlich weiter, eher in Richtung der Politik, vielleicht bundesweit. (HSL7: 66)

Aus Sicht der Hochschulleitungen müssten die HAWs ihre Stärken neu aufzeigen (dieser Gedanke ist auch auf andere Hochschularten übertragbar). Einzelne Hochschulleitungen betonten die Rolle der HAWs hinsichtlich ihrer Nähe zu gesellschaftlichen Fragestellungen, da sie sich mit Forschungen zu gesellschaftlich relevanten Rahmenbedingungen beschäftigten und im Austausch mit entsprechenden Stakeholdern stehen. 
Wir haben einen sehr, sehr engen Kontakt und wir haben neben aller Wissenschaftlichkeit und Forschungsaktivitäten in allen möglichen verschiedenen Bereichen die Bodenhaftung, wenn ich das sagen darf. Ja, also wir sind überhaupt nicht im Turm und schon gar nicht im Elfenbeinturm, sondern wir sind durch unsere Fragestellungen, die wir aus der Gesellschaft kriegen, bearbeiten und in die Gesellschaft zurückgeben, mit der Gesellschaft verbunden. (HSL11: 50)

Der Bildungsauftrag der HAWs ist im Vergleich zu den Universitäten ein anderer, die Studierendenschaft ist heterogener. An die HAWs kommen Studierende mit unterschiedlichen Hochschulzugangsberechtigungen, die Bildungsaufsteiger*innen müssen beispielsweise verstärkt mitgenommen werden und das Studium so gestaltet werden, dass die unterschiedlichen Studierendengruppen Berücksichtigung finden. Die regionale Verankerung der HAWs bildete ebenfalls ein Themenfeld einiger Hochschulleitungen. Die Hochschulen sind meist im Kontakt mit Unternehmen und Institutionen in ihrer jeweiligen Region. Die zukünftige regionale Rolle müsste reflektiert und ggf. neugestaltet und ausgerichtet werden. Hier ist auch die Arbeitsmarktsituation der jeweiligen Region von Bedeutung und inwiefern die Hochschulen zukünftig im Bereich der Weiterbildung von Fachkräften eine verstärkte Rolle übernehmen oder wie sich die Hochschulen hier ausrichten wollen.

Also gerade, weil wir eben stark regional verankert sind, versuchen wir natürlich ganz stark, solche regionalen Themen auch bei uns zu verankern an der Hochschule und das machen wir auch sehr gut. Wir haben enge Bindungen in die regionale Wirtschaft. Wir erfüllen da so den regionalen Fachkräftebedarf sehr stark. (HSL7: 74)

HAWs zeichnen sich durch eine starke Praxisorientierung, das Lernen in Laboren und gemeinsames Projektlernen aus. Dieses Merkmal kann insbesondere durch die Vor-Ort-Lehre bedient werden. Durch die Umstellung auf die digitale Lehre und den damit verbundenen Wegfall des Erlebens vor Ort und des Lernens in kleinen Gruppen standen HAWs vor der Herausforderung, ihrem Zusammenhalt und ihrer Praxisorientierung gerecht zu werden. Vor dem Ausbruch der Pandemie waren die Studierenden und Lehrenden über die kurzen Wege innerhalb der Hochschule miteinander verbunden. Die HAWs stehen daher vor der Anforderung, das Gemeinschaftsgefühl vor Ort wiederherzustellen, auch die Lehrenden wieder zurück an die Hochschule zu 
bringen, das Wir-Gefühl, das Näher-dran-Sein, insgesamt das HAWFeeling wieder zu stärken. Als Ideen wurden „Welcome-Back“-Events oder Aktionen auf dem Campus genannt. Es brauche laut den Hochschulleitungen die Präsenz an der Hochschule, damit ein Studienerlebnis stattfinden kann und die Studierenden gerne und begeistert studieren. Die Hochschulleitungen waren sich einig, dass die HAWs sich weiterhin als Präsenzhochschule definieren, aber mit der Erweiterung von integrierten digitalen Formaten. Hierzu gehörte beispielsweise die Diskussion über Wohnkonzepte für Studierende, wenn die Vor-Ort-Präsenz reduziert werde. Aus Sicht der Hochschulleitungen braucht es einen Diskurs zur Weiterentwicklung der HAWs, für den unterschiedliche Aspekte genannt wurden: Kompetenzentwicklung der Studierenden hinsichtlich der zukünftigen Anforderungen des Arbeitsmarktes, die Weiterentwicklung der bisherigen Stärken und des eigenen Selbstverständnisses, die Entwicklung einer hybriden Studienform, die Präsenz und Digital verbindet, die Hochschulprozessentwicklung und als letzter Punkt die Sichtbarkeit der HAWs. 


\section{Zugang 2: Die Corona-Semester aus Sicht der Lehrenden und Studierenden}

Im fünften Kapitel liegt der Fokus auf den Perspektiven von Professor*innen, Lehrbeauftragten und Studierenden (s. Abbildung 13). Auf der Grundlage der Ergebnisse aus digitalen Fokusgruppen wird dargestellt, wie die Corona-Semester von Lehrenden und Studierenden tatsächlich erlebt wurden. Es geht konkret um die Frage, was innerhalb des in Kapitel 4 aufgespannten Kontingenzraums geschah. Zunächst werden im Folgenden die Teilforschungsfragen und das methodische Vorgehen dargestellt. Daran schließt die Beschreibung der konkreten Lehr-/Lernsituation (s. Kapitel 5.2) und der Gesamtsituation (s. Kapitel 5.3) an. In Kapitel 5.4 werden daran anknüpfend die Chancen post Corona wiedergegeben, die die Fokusgruppen-Teilnehmenden selbst identifizierten.

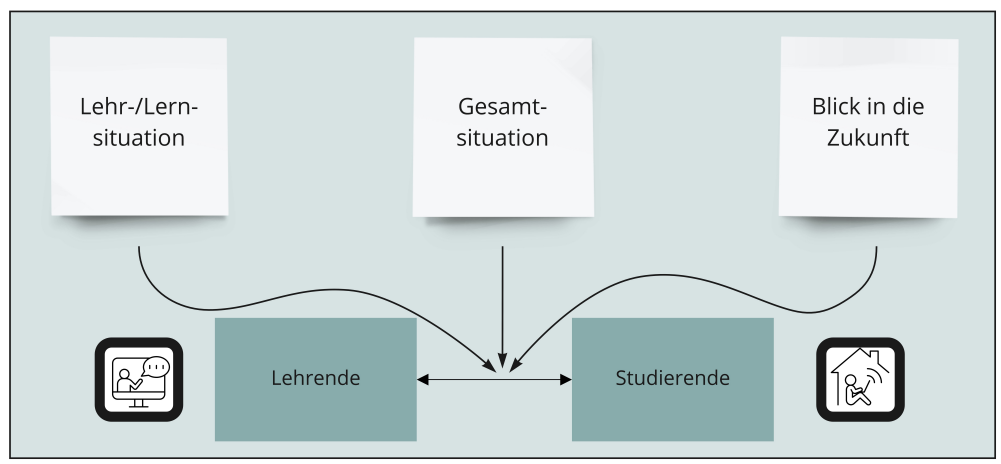

Abbildung 13: Aufbau Forschungszugang 2

Jedes Unterkapitel ist nach dem gleichen Schema aufgebaut: Zunächst werden die Ergebnisse aus den Fokusgruppen der Lehrenden ggf. inklusive Besonderheiten der Lehrbeauftragten beschrieben, anschließend die Ergebnisse aus den Fokusgruppen der Studierenden ggf. in- 
klusive Besonderheiten der Erst- und Zweitsemester und abschließend werden die Ergebnisse zusammengeführt.

\subsection{Untersuchungsgegenstand und methodische Vorgehensweise}

Im Forschungszugang 2 wurde die Perspektive von Lehrenden und Studierenden auf die vergangenen Corona-Semester in den Blick genommen. Dabei waren folgende Teilforschungsfragen leitend (s. Abbildung 14).

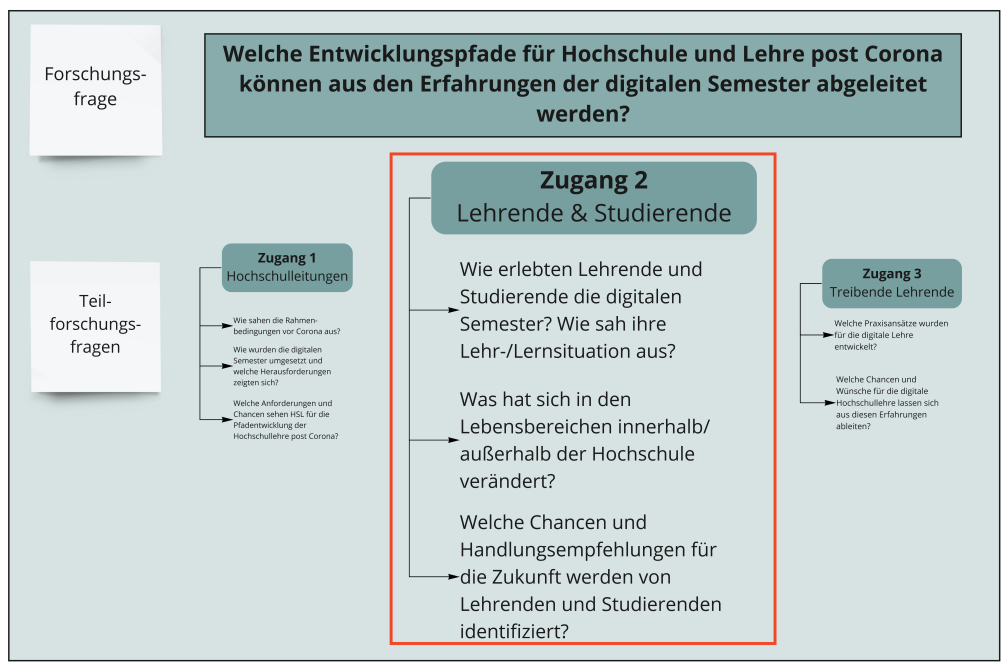

\section{Abbildung 14: Teilforschungsfragen Zugang 2} im Gesamtzusammenhang

Die Teilnehmenden der Fokusgruppen wurden nach Fächerclustern ausgewählt. In Anlehnung an die zehn Studienbereiche, die im Hochschulkompass der Hochschulrektorenkonferenz angeführt sind ${ }^{5}$, wurden sechs unterschiedliche Fächercluster gebildet, denen sich die im Sample vertretenen HAWs zuordnen ließen: Gestaltende Künste,

5 www.hochschulkompass.de/home 
Naturwissenschaften und Lebens- und Umweltwissenschaften, Wirtschafts- und Sozialwissenschaften, Ingenieurwissenschaften sowie Informatik und technische Prozesse. Aus den Fächerclustern und den methodischen Empfehlungen von Krueger und Casey (2015) zur Fokusgruppen-Methode ergaben sich 15 Gruppen mit insgesamt 67 Teilnehmenden (davon waren 26 Studierende, 31 Professor ${ }^{*}$ innen, 10 Lehrbeauftragte). Im Zeitraum von Mitte Februar bis Anfang März 2021 wurden in jedem hochschulübergreifenden Fächercluster je eine Fokusgruppendiskussion mit Studierenden und eine Fokusgruppe mit Lehrenden geführt. Zusätzlich wurden zwei Fokusgruppen mit Lehrbeauftragten und eine mit Studierenden des zweiten Semesters geführt. Zwei fächerübergreifende Fokusgruppen wurden ebenfalls in die Auswertung miteinbezogen.

Die Methode der Fokusgruppen ist gekennzeichnet durch eine Mischung aus Gruppeninterview und Gruppendiskussion, d.h. es werden Fragen gestellt und gleichzeitig die Teilnehmenden dazu motiviert, selbst ins Gespräch zu kommen, sich also gegenseitig zu ergänzen oder zu kommentieren (Krueger \& Casey, 2015). Hier in Zugang 2 wurden je eine Moderatorin und eine Co-Moderatorin eingesetzt, sodass alle für diese Untersuchung wichtigen Aspekte aufgegriffen werden konnten und bei etwaigen Verbindungsstörungen immer eine Moderatorin einspringen konnte. Die Fokusgruppen wurden mit Video aufgezeichnet, damit auch nonverbale Signale in die Transkriptionen aufgenommen werden konnten. Nicken, Lachen und Daumen hoch waren die von den Teilnehmenden am häufigsten gezeigten nonverbalen Signale.

Um den Leitfaden für die Fokusgruppen zu erstellen, wurden vorab die bereits vorhandenen Studien zu den Corona-Semestern gesichtet. So konnten wichtige wiederkehrende Themen herausgearbeitet werden, die zu Clustern zusammengefasst und mit zusätzlichen Schwerpunkten zu einer umfänglichen Leifadenstruktur entwickelt wurden.

Der Leitfaden war in folgende Themenbereiche gegliedert:

- Vorstellungsrunde

- Warm-up-Frage zu Lehr-/Lernort

- Themenfelder zur Diskussionsvertiefung

- Im Studium / In der Lehre

- Kommunikation und Information 
- Private Situation

- Gesamtsituation

- Blick in die Zukunft

Im Hinblick auf das Themenfeld Studium und Lehre wurde die konkrete Lehr-/Lernsituation beleuchtet, insbesondere aus einem Vergleich mit Zeit vor Corona heraus. Ferner wurden weitere die Lehr-/ Lernsituation betreffende Themen, darunter der Workload, die Selbstorganisation, das An- und Ausschalten der Kamera sowie andere technische Aspekte angesprochen. Im Themencluster zum Austausch standen Fragen nach dem formellen und dem informellen Austausch während und außerhalb der Veranstaltungen im Mittelpunkt. Zur Privatund Gesamtsituation adressierten die Fragen die konkrete Wohnsituation, das Leben außerhalb der Hochschule sowie den Ausgleich vom Lehren bzw. Lernen. In diesen Themenbereichen wurde zusätzlich zur Ist-Beschreibung auch auf Herausforderungen und Chancen bzw. Vorteile geblickt. Im letzten Abschnitt des Leitfadens richtete sich die Aufmerksamkeit auf die unmittelbare Zukunft des nächsten Semesters (SoSe 2021), aber auch auf die Post-Corona-Zeit. Darin wurden die Gruppen auch danach gefragt, was sie selbst bezüglich des Lehrens und Lernens aus den Corona-Semestern lernen sollten.

Für die Fokusgruppen mit den Studierenden und Lehrenden wurde jeweils ein Leitfaden entwickelt (s. Anhang 2 und Anhang 3). Die leitfadengestützten digital geführten Fokusgruppen wurden mithilfe kategorienbasierter Verfahren in Orientierung an die inhaltlich strukturierende qualitative Inhaltsanalyse nach Kuckartz (2018) ausgewertet.

\subsection{Konkrete Lehr-/Lernsituation}

Das hier folgende Abschnitt zeichnet nach, wie die konkrete Lehr-/ Lernsituation aus Sicht von Lehrenden und Studierenden erlebt wurde. Hierzu gehört die Realisierung sowohl synchroner Veranstaltungen als auch asynchroner Formate, wie zum Beispiel Videos und Aufzeichnungen. Es geht im Folgenden um die konkrete Umsetzung der digitalen Lehre, deren Wahrnehmung und die daraus resultierenden Herausforderungen. 


\section{a. Perspektive der Lehrenden}

Also, wo ich halt ein großes Problem tatsächlich sehe, das ist ja auch schon angesprochen worden, ist, dass wir als Lehrende halt keine direkte Rückmeldung ((Herr B, Herr C, Herr D nicken)) bekommen im Hörsaal. $\mathrm{Da}$ sehe ich halt, wenn mir die Leute wegschlafen ((Herr C, Herr D lachen $)$ ), ((Herr B nickt $)$ ) und irgendwie sich mit anderen Dingen beschäftigen ((Herr B, Herr D nicken)), ((Herr C lacht)), dann kann ich versuchen, die wieder zurückzuholen. So starre ich halt auf meine grauen Kacheln, weil die bei uns auch in der Regel die Kamera ausgeschaltet haben, damit auch die Leute, die schwächer bandbreitenmäßig angebunden sind, noch eine Chance haben teilzunehmen, kriege ich das natürlich nicht mit. Was bei uns sehr gut funktioniert, ist tatsächlich die Nutzung des Chats. (FGL6: 30)

So beschrieb ein ${ }^{\star}$ e Lehrende ${ }^{\star} r$ die Lehrsituation im Corona-Semester und brachte die Erfahrung der digitalen Lehre auf den Punkt. Zuerst galt es, die technische Umstellung der Lehre von heute auf morgen zu bewerkstelligen, und dies mittels unterschiedlicher Erfahrungen, wie viele Lehrende in den Fokusgruppen berichteten: „Also den Übergang selbst empfand ich jetzt auch nicht als schwierig, das hat sich dann relativ schnell auch im Learning by Doing gezeigt, was halt funktioniert und was nicht" (FGL3: 40). Beschrieben wurde dabei auch, dass sie erst einmal die eigenen Hemmschwellen überwinden mussten.

Ich war am Anfang eigentlich der Meinung, das wird überhaupt nicht funktionieren können, und war dann überrascht, wie schnell es dann doch ging. Und muss auch dazu sagen: Dank der großen Unterstützung, die wir von der Hochschule bekommen haben, technische und didaktische Hilfestellungen. (FGL1: 23)

\section{Bei der Umsetzung der digitalen Lehre berichteten die Lehrenden} über unterschiedliche Vorgehensweisen. Einige Lehrende erklärten, dass sie ähnlich wie in Präsenz unterrichteten, wohl wissend, dass dies didaktisch keine gelungene digitale Lehre sei. Sie wollten in dem Fall zunächst abwarten, wie schnell wieder auf die Präsenzlehre umgestellt werden konnte. Andere nutzten die digitalen Gruppenräume, die sogenannten Breakout Rooms ${ }^{6}$, um Lerngruppen zu bilden. Ferner

6 Breakout Rooms sind separate Teile eines Internet-Meetings, in dem kleine Gruppen diskutieren können. Nach der Diskussion kehren die Teilnehmenden in das Hauptmeeting zurück. (https://dictionary.cambridge.org/de/worterbuch/englisch/b reakout-room) 
nahmen manche Lehrende Videosequenzen bzw. vertonte PowerPointPräsentationen auf oder nutzten frei zugängliche Filmsequenzen als thematischen Input. Darüber hinaus gab es diejenigen, die ihre Lehre auf hybride Formate umgestellt hatten. Dabei wurden auf der einen Seite die meist schon vorhandenen Lernplattformen ILIAS, Moodle oder eine hochschuleigene Variante genutzt oder die Ablage der Lehrmaterialien erfolgte direkt in den Videokonferenzsystemen. Mehrheitlich wurde über E-Mail und Chat der Videokonferenzsysteme oder der Lernplattformen mit den Studierenden kommuniziert. Als hilfreich empfanden die Lehrenden die verschiedenen Unterstützungsleistungen, insbesondere auch von Tutor ${ }^{\star}$ innen. Die Art der digitalen Lehre variierte zwischen den befragten Lehrenden, mitunter auch abhängig von dem Fach und der Gruppengröße: „Je mehr Teilnehmer dabei sind, obwohl das ja online gar keinen Unterschied eigentlich macht, ja, aber je länger die Liste wird, desto inaktiver wird der ganze Haufen“" (FGL3: 46). Die Lehrenden waren sich des Weiteren darin einig, dass die Lehrveranstaltungen insgesamt digital viel besser durchgeplant werden mussten, denn die Vorbereitung war zeitintensiver und die Lernschritte, die sonst in der Präsenzlehre über den physischen Austausch zustande kommen, mussten größtenteils vorab überlegt werden.

Als Stolpersteine erwiesen sich für die Lehrenden die unklaren Strukturen bei der Anwendung unterschiedlicher digitaler Werkzeuge, Lernplattformen und Videokonferenzsysteme. Es war für sie oftmals unklar, wie weit ihre Handlungsspielräume gingen. Einige nutzten bestimmte digitale Werkzeuge, mussten aber später wieder auf andere umstellen, da es dafür Vorgaben vonseiten der Hochschule gab: „Jede Hochschule muss anscheinend sein eigenes Jura-Süppchen basteln. Was ist erlaubt, was ist nicht erlaubt" (FGL6: 61). Es bestand ein stetiges Gefühl der Unsicherheit bezüglich des Datenschutzes und der damit verbundenen Regelungen bei der Auswahl des Videokonferenzsystems (kommerziell oder nicht-kommerziell) sowie bei Videoaufzeichnungen und Kameranutzung.

Der Workload in den Corona-Semestern wurde als deutlich höher beschrieben. Die Umstellung auf die digitale Lehre beinhaltete Zusatzarbeit, die meist am Abend oder am Wochenende stattfand: „Also ich würde mal sagen, im Schnitt arbeite ich von 9 Uhr bis 24 Uhr jetzt. Das aber regelmäßig, ja? Also das ist wirklich verändert" (FGL2: 
68). Und jedes Semester kamen wieder andere Veranstaltungen bzw. neue Lehrinhalte hinzu, die es digital neu aufzubereiten galt, d.h. der Prozess war nach dem ersten Corona-Semester nicht abgeschlossen. Im Wintersemester 2020/2021 musste auf Onlineprüfungsformate umgestellt werden, an manchen Hochschulen war das bereits im Sommersemester 2020 ein Thema. Diese Zusatzbelastung war aus Sicht der Lehrenden auf Dauer so nicht zu bewerkstelligen: „Ja, ich arbeite sehr viel nachts und am Wochenende, aber ich möchte nicht, dass das mein neues Dauerleben ist. Das wird nicht funktionieren“" (FGL3: 95). Insbesondere beim Herstellen von guten Videos kostete es viel Zeit, bis eine Lernsequenz vorbereitet und korrekt aufgenommen war: „Also die Videos zu machen für also zehn Minuten eine Stunde, das ist schon gut gerechnet, das ist eher mehr. Also wenn man ein hochqualitatives Video machen möchte ((Herr F nickt)), dann ist das eher mehr“ (FGL5: 32). Dies mache durch den erhöhten Aufwand daher nur Sinn, wenn diese Videos auch in den folgenden Semestern eingesetzt werden könnten.

Ein großer Diskussionspunkt für die Lehrenden bezog sich auf den reduzierten Austausch und die veränderte Interaktion zwischen Lehrenden und Studierenden, sowohl innerhalb als auch außerhalb der Lehrveranstaltungen. Es wurde dabei als aufwändig empfunden, die fehlende physische Interaktion digital herzustellen, insbesondere die zwischenmenschliche Ebene ließ sich schwierig umsetzen. Die direkte Rückmeldung, die Lehrende sonst in Vor-Ort-Lehrsituation über Kopf schütteln, Augen verdrehen oder Gähnen erhalten, fehlte in der digitalen Situation. Diese non-verbalen Signale fehlte den Lehrenden dann besonders, wenn es darum ging, die Geschwindigkeit anzupassen oder entsprechende Wiederholungen einzubauen. Die Lehrenden hatten zudem den Eindruck, dass die Studierenden sich schneller inhaltlich ausklinkten und dass sie selbst gelegentlich nicht mitbekamen, ob diese wirklich mitdenken oder nur online seien.

Probleme haben die Studierenden, gerade wenn sie ihre Kamera nicht einschalten, oder ich habe das Problem, dass mir das Feedback fehlt bei den Verständnissachen. ((drei Personen nicken)) Also, wenn ich in der Vorlesung bin, dann sehe ich einfach in die Augen, ob sie abgeschweift sind, ob sie gelangweilt sind, ob sie noch dabei sind, ob ich sie langweile oder ob ich sie überfordere. (FGL3: 42) 
Aus Sicht der Lehrenden liefen dagegen Vorlesungen in großen Gruppen, z.B. Grundlagenvorlesungen, digital störungsfreier und effektiver $\mathrm{ab}$, da die sonstige Unruhe und Ablenkungen der Präsenzveranstaltung nicht vorhanden waren.

Ja, es gibt Vorlesungen, zum Beispiel in Mathe, die ist tatsächlich, finde ich, besser, als es in Präsenz war, weil, auch da habe ich so 130 demotivierte Studierende, die normalerweise am Mittwochnachmittag um 13:45 Uhr nach dem Suppenkoma, ja, eigentlich immer nur Lärm machen. (FGL3: 34)

Die ausgeschaltete Kamera als Interaktionshemmnis bildete einen weiteren großen Diskussionspunkt in allen Fokusgruppen. Die Lehrenden fühlten sich oftmals alleine, hatten das Gefühl, dass kein tatsächliches Gegenüber existierte, wie es eine Lehrende hier auf den Punkt brachte: „Eigentlich führt man Selbstgespräche und ich brauche die Rückmeldung. Also, Lehren heißt für mich Interaktion und nicht ich rede“ (FGL1: 29). Darüber hinaus wurden die Vorlesungen als viel anstrengender und ermüdender wahrgenommen: „Es ist für mich in einer Vorlesung viel anstrengender, 90 Minuten ((Herr E nickt)) in diesen Bildschirm reinzugucken und 50 schwarze Bobbel zu sehen" (FGL5: 32). In kleineren Gruppen klappte es weiterhin sehr gut, doch manche Lehrenden berichteten, dass sie sehr nachdrücklich, teils auch etwas spaßig, jedes Mal das Anschalten der Kamera einforderten und es dann auch irgendwann funktionierte. Für manche war die Kamera zum Unterrichten essenziell, andere hatten sie zum Teil selbst aus, wenn sie beispielsweise auf dem Bildschirm eine Aufgabe rechneten, damit sich alle auf die Aufgabe konzentrieren konnten. Die Studierenden wurden fallweise über die Verteilung von zusätzlichen Moderations- oder Referentenaufgaben aktiviert, dabei hielten manche Lehrende den Austausch in der digitalen Situation genauso für möglich wie in der Präsenzlehre: „Wenn ich also kleinere Seminare habe mit acht Teilnehmern, da ist die ganze Zeit Diskussion“ (FGL3: 46). Im Digitalen herrschte eine serielle Diskussionskultur, da nicht mehrere Personen gleichzeitig sprechen könnten. Impulsive oder spontane Äußerungen, von denen Diskussionen gerade auch leben, gingen teils verloren, da sich alle an die Meldungsreihenfolge halten mussten.

Der Austausch zwischen Kollegæinnen, aber auch zwischen Lehrenden und Studierenden wurde unterschiedlich erlebt. Auf der einen 
Seite wurde die Auffassung vertreten, dass informelle und persönliche Gespräche digital nicht funktionierten, auf der anderen Seite wurde von positiven Beispielen (digitalen Kaffee- und Austauschrunden, digitalen Jour fixe mit den Dekan*innen) berichtet, die wiederum zeigten, dass auch im digitalen Raum ein zwischenmenschlicher Austausch möglich war. Für die Kommunikation mit den Studierenden richteten einige Lehrende Chatkanäle bzw. Chatgruppen ein oder nutzten dafür die Forumsfunktion auf der Lernplattform. Hier kam Austausch zustande. Manche boten zusätzliche digitale Sprechstunden oder offene Termine ohne vorgegebenes Thema an. Ebenfalls ermöglichte eine längere Anwesenheit vor und nach der digitalen Veranstaltung im virtuellen Klassenraum den Austausch. Mittels digitaler Kommunikationskanäle konnten die Studierenden schneller und besser erreicht werden und weitere Personen konnten leicht dazugeschaltet werden. Andererseits wurde die Kommunikation als aufwändiger erlebt, da entsprechende Termine vereinbart werden mussten oder der Informationsfluss nicht stattfand. Außerhalb der Lehrveranstaltung fehlte der informelle Austausch mit Studierenden, Kolleg*innen und Lehrenden anderer Studiengänge und Fakultäten, da die zufälligen Begegnungen am Kaffeeautomaten, auf dem Flur oder in der Mensa wegfielen: „Was fehlt, mir persönlich, ganz massiv, ist dieses Kaffeegespräch. Also was überhaupt nicht mehr geht, ist, auf dem Flurfunk etwas aufschnappen“ (FGL6: 55). Man erfuhr nicht mehr die persönlichen und privaten Dinge voneinander: „Natürlich können wir viele Dinge, man kann Geburtstag online feiern und, und, und. Aber es sind ja alles Krücken“ (FGL5: 40). Andere Lehrende vermissten den Raum mit Kolleg*innen, um spontan Neues zu entwickeln, was sonst im informellen Gespräch wie zufällig entstand.

Weiterhin bestand auch Unsicherheit, ob manche Studierende im digitalen System durch die Anonymität verloren gingen, da sie in den synchronen Veranstaltungen weder Mikrofon noch Kamera anschalteten und damit abtauchten bzw. nicht erreichbar waren: „Ich erlebe diese Zeit als eine sehr starke Vereinsamung, auch von den Studierenden untereinander, und finde da überhaupt nichts schönzureden“ (FGL4: 87). Zum Teil bekamen die Lehrenden dadurch Persönliches von Studierenden, wie z.B. private Themen oder Probleme, noch weniger mit. Auch das Peer-to-Peer-Lernen zwischen den Studierenden 
fehlte: „Studierende lernen voneinander mindestens so viel wie von den Professoren" (FGL4: 86). Lehrende berichteten, dass Studierende andererseits auch von sich aus neue Austauschmöglichkeiten schufen, indem sie beispielsweise über einen Discord-Server einen digitalen Kanal neben dem Lernen anschalteten und dadurch in Kontakt waren.

\section{b. Perspektive der Studierenden}

Also gerade die Ablenkung ist natürlich immens. Man neigt dazu, unglaublich viele Dinge noch nebenher zu machen, wo man in der Vorlesung gar nicht darauf käme, keine Ahnung, irgendwie auf Amazon noch was bestellt nebenher, weil es einfach so verlockt und so einfach überhaupt kein quasi äußeren ((macht Geste für Anführungszeichen)) Druck jetzt gäbe, sich da auch irgendwie auf etwas zu konzentrieren. Weil, ich meine, wenn ich irgendwie in einem Hörsaal sitze, na ja, dann bin ich ja schon da, dann beschäftige ich mich auch damit. (FGS5: 18)

Die konkrete Lernsituation variierte bei den Studierenden deutlich, was sich ab Beginn des Sommersemesters 2020 über den gesamten retrospektiv betrachteten Zeitraum der ersten beiden Corona-Semester zog. So startete das erste Corona-Semester teilweise pünktlich, teilweise verschob es sich bis zu vier Wochen. Weitere Varianzen der konkreten Lernsituation der Studierenden werden im Folgenden genauer beschrieben.

Sowohl in der Lehre als auch für das digitale Lernen wurden unterschiedlich viele digitale Werkzeuge genutzt. Teilweise waren die Studierenden mit bis zu fünf neuen und zusätzlichen Videokonferenzsystemen und Austauschplattformen konfrontiert (WebEx, Zoom, MS Teams, Discord, Slack). Die Lernplattformen ILIAS oder Moodle wurden mehrheitlich bereits vor Corona genutzt, sodass Studierende zu Beginn der Corona-Semester mit bis zu sieben (inklusive E-Mail) unterschiedlichen digitalen Werkzeugen jonglieren mussten. Synchrone und asynchrone Formate wie Onlinevorlesungen oder -vorträge, virtuelle Frage- und Sprechstunden, digitale Gruppen- und Projektarbeiten, Aufzeichnungen der Veranstaltungen oder Videos gestalteten die Lernsituation der befragten Studierenden.

In synchronen Veranstaltungen war die Gefahr der Ablenkung allgegenwärtig. Anders war es im Vorlesungs-/Seminarraum, denn dort befanden sich alle physisch vor Ort, wodurch ein ablenkungsfreier 
Rahmen gegeben war und die Möglichkeit zur direkten Interaktion mit den Lehrenden und den Kommiliton*innen bestand. Dies fehlte in den Corona-Semestern. In der digitalen Lehre waren Lehrende zum "Alleinunterhalter“ (FGS2: 57) geworden, so manche ${ }^{\star} r$ Studierende. Andere berichteten dahingegen von Lehrenden, die die Studierenden miteinbezogen und durch ihr lockeres Verhalten das Lernen erleichterten. Kleine Gruppen und das Anschalten der Kamera halfen, die Ablenkung zu reduzieren. Auch die Gruppen-/Projektarbeiten waren für Studierende digital schwierig zu gestalten, was wiederum in der Präsenzlehre deutlich anders war: „Jede Gruppe ist dann im Zeichensaal verteilt, man kann da ein bisschen rumlaufen und man sieht die Entwürfe und Projekte von den anderen Gruppen und findet das super spannend, holt sich da seinen Input" (FGS1: 30). Nun mussten die Gruppen mit viel Koordinationsaufwand einen Termin finden und wenn dieser stattfand, hinderten Verbindungsprobleme das gemeinsame Arbeiten und Missverständnisse entstanden.

Ich glaube, was mir eben auch total gefehlt hat, war das Lernen jetzt zusammen, weil, ich hatte immer so eine Gruppe. Wir haben uns immer am gleichen Platz in der Bibliothek getroffen, wir haben zusammen Mittagspause gemacht, Kaffee geholt usw., und eben dieses: An der Uni einfach sein, also einfach dorthin gehen und dann trifft man mal den und mal den und dann quatscht man hier mal noch. (FGS2: 109)

Das Zitat zeigt, wie der Hochschulalltag vor Corona gestaltet wurde. Durch das Digitale der Lehre in den Corona-Semestern fehlte demnach das Peer-to-Peer-Lernen komplett, sodass gegenseitige Hilfestellungen während und außerhalb der Veranstaltungen sowie der Vergleich mit anderen fehlten: „Ich glaube, da schwingt einfach so eine gewisse Demotivation mit einher bei dieser Onlinelehre, einfach weil man vieles alleine vor einem Bildschirm macht. Das ist so, als würde man sich ein YouTube-Tutorial anschauen" (FGS1: 71). Hier wurde sichtbar, dass bei den Studierenden ein Gefühl von Einzelkämpfertum im anonymen Raum entstand. Doch diese unterhielten sich während der synchronen Veranstaltungen über eine weitere Austauschplattform, um entstehende Fragen direkt zu klären. Hier funktionierte das Peer-to-Peer-Lernen.

Darüber hinaus wurde beschrieben, dass bei einigen Lehrenden eine didaktische Anpassung an das digitale Setting fehlte und lediglich eine 
Eins-zu-eins-Übertragung der Lehre von Präsenzlehre ins Digitale geschah. Das führte dazu, dass die Vorlesungen als weniger spannend wahrgenommen wurden, also die Abwechslung bei digitalen Veranstaltungen fehlte. Auch fühlten sich einige Studierende nicht genug vonseiten der Lehrenden unterstützt, was sich im fluchtartigen Verlassen des digitalen Raums seitens der Lehrenden unmittelbar nach der Veranstaltung und deren schwieriger Erreichbarkeit zeigte. Darüber hinaus gingen einige Lehrende nicht auf das Feedback der Studierenden ein, wie das folgende Zitat zeigt: „Und sagen wir, manche Leute haben eine gewisse Beratungsresistenz. ((alle lachen, Herr $\mathrm{H}$ zeigt beide Daumen hoch)) Die nehmen Vorschläge von Studentenseite jetzt auch nicht wirklich gut an" (FGS3: 65).

Der gestiegene Workload, d.h. die Zeit, die für das Studium verwendet werden musste, war für viele Studierende eine Herausforderung. Der erhöhte Workload wurde von den Studierenden unter anderem auf die vermehrten Abgaben während des Semesters zurückgeführt, weil die Prüfungsleistungen an die Corona-Situation angepasst werden mussten: „Ich hatte mehr Abgaben unter dem Semester. Also ich hatte zwei Projektarbeiten und die wären beide, meine ich, keine Projektarbeiten gewesen, wäre es in Präsenz" (FGS2: 42). Als weiterer Grund wurde der fließende Übergang zwischen den Veranstaltungen, Terminen und Lernorten genannt, der vor Corona durch kleine Pausen, Raumwechsel oder Gebäudewechsel und nun nur durch wenige Klicks gekennzeichnet war.

Das Selbststudium beschrieben die Studierenden ebenfalls als herausfordernd, da durch den nahtlosen Übergang von einer zur anderen Veranstaltung und den höheren Workload die Selbststrukturierung eine wichtige Rolle spielte. Durch die Flexibilität der digitalen Lehre stieg die Prokrastination, es wurden mehr Selbstdisziplin und Eigenverantwortung gefragt und der Selbstdruck stieg. Darüber hinaus mussten teilweise Videos und Aufzeichnungen mehrfach angeschaut werden, um den Inhalten gänzlich zu folgen. Einigen Studierenden gelang es, sich zu strukturieren, anderen gelang es nicht.

Der Austausch, der vor Corona auf dem Flur, vor und nach der Veranstaltung und in der Mensa stattfand, musste in den vergangenen Corona-Semestern aktiver gesucht und gestaltet werden. Austausch 
und Interaktion zwischen Lehrenden und Studierenden fand innerhalb der Veranstaltungen in unterschiedlichem Maße statt. Durch die erschwerte Interaktion fehlte den Studierenden die Nähe zu den Lehrenden, die eine Hochschule für Angewandte Wissenschaften auszeichnet. Auch das Gemeinschaftsgefühl und das Familiäre fehlten in den Corona-Semestern, da eben Flurgespräche und gemeinsame Kaffeeoder Mittagspausen mit Lehrenden und Kommiliton*innen weggefallen waren.

Das fehlt mir auf jeden Fall. Und eben schon auch das irgendwie in der Mittagspause oder so sich da überhaupt irgendwie Belanglosigkeiten auszutauschen, einfach wie geht es mir gerade, was beschäftigt mich sonst oder was passiert nebenher. (FGS4: 75)

Und was vermisse ich im Online ganz viel: Die Zwischenkommunikation auf dem Flur. ((alle nicken)). (FGS3: 67)

Das Thema Kamera war nicht nur im Zusammenhang mit dem Lernen in Gruppen, sondern auch für die Interaktion mit den Lehrenden in den Veranstaltungen ausschlaggebend. Wenn die Kameras angeschaltet waren, kam es zur Interaktion und zum Austausch. Für die befragten Studierenden war klar, dass es für Lehrende schwierig war, mit ihnen in Kontakt zu treten, wenn sie selbst die Kameras und Mikrofone ausgeschaltet ließen. Das führte auch dazu, dass in den Veranstaltungen kaum Fragen vonseiten der Studierenden gestellt wurden oder gestellte Fragen nicht beantwortet wurden.

Und es gibt halt so bei uns so die drei, vier Leute, die dann halt immer mitmachen und der Rest ist halt entweder gar nicht anwesend oder ((lacht)) ja, also voll auf dieses In-den-schwarzen-Bildschirm-Hineinreden, das gefällt den Professoren halt auch nicht. Und deshalb verstehe ich auch, dass es irgendwo von beiden Seiten aus nicht funktionieren kann. (FGS7: 96)

Warum sie selbst die Kamera nicht anmachten, begründeten einige dadurch, dass sie sich beobachtet fühlten oder Verbindungsprobleme hatten. Ein ${ }^{\star}$ S Studierende ${ }^{\star} r$ beschrieb die Situation und das Gefühl des Beobachtetwerdens wie folgt:

Und wenn man sich mal irgendwie einen Kaffee holen will oder mal einfach auf die Toilette muss, neigt man immer dazu, die Kamera gleich auszumachen, weil man es irgendwie ganz komisch findet, den Raum zu verlassen und dann da irgendwie so einen leeren Stuhl zu hinterlassen in 
dem Kamerabild, was im Vorlesungssaal ja eigentlich auch ganz normal wäre, dass man da mal rausgeht. (FGS1: 62)

Für die Studierenden und vor allem auch die Gruppe der Zweitsemester war das Fehlen des Kennenlernens der Kommiliton`innen vor Ort ein großes Problem. Denn mit dem gegenseitigen Kennenlernen ginge auch das Kennenlernen der Strukturen der Hochschulen und der Lehrenden einher. Auf Unbekannte zuzugehen, war im Digitalen noch schwieriger als in Präsenz.

Ja, du musstest wirklich AKTIV jemanden anschreiben oder jemanden über Teams anrufen, was dann halt auch irgendwie komisch ist von einer Person, die du irgendwie nur virtuell ((Frau G, Herr I nicken)) bisher gesehen hast, noch kein privates Gespräch oder hier mal eine WhatsAppNachricht ((atmet tief)). (FGS3: 58)

Auch spontane Begegnungen fielen in den Corona-Semestern weg, sodass digitale Zusammentreffen geplant werden mussten. Das aktive Organisieren dieser Treffen kostete jedoch Energie und Motivation: „Also auch wenn wir jetzt zum Beispiel in einer Zoom-Veranstaltung mal eine Pause haben. Aber da kommt man lange nicht so in dieses, ja, spontane Gespräch, wie man es sonst auf dem Gang oder im Vorlesungssaal hätte“ (FGS1: 32). Studierenden fehlten der Austausch und die Interaktion sowohl mit Lehrenden als auch mit den Kommiliton ${ }^{\star}$ innen und Freund ${ }^{\star}$ innen außerhalb der synchronen Veranstaltungen.

\section{c. Zusammenführung}

Zusammenführend wird deutlich, dass sowohl bei den Lehrenden als auch bei den Studierenden die Lehr-/Lernsituation jeweils unterschiedlich erlebt und bewältigt wurde. Einige kamen besser mit der digitalen Situation zurecht, andere konnten sich schlechter darauf einlassen. Somit zeigte sich in den Fokusgruppen ein differenziertes Bild in Bezug auf die wiederkehrenden, beidseitig adressierten Aspekte (s. Abbildung 15). 


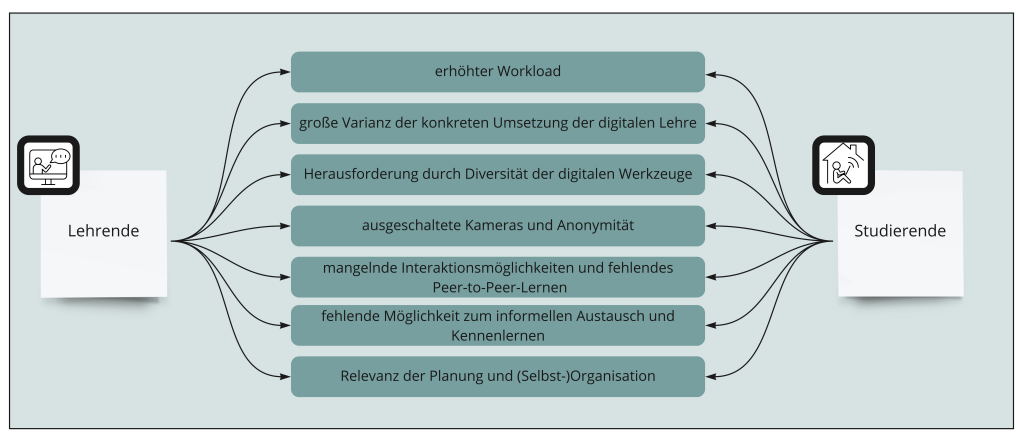

Abbildung 15: Aspekte für den Bereich Lehr-/Lernsituation

Sowohl Lehrende als auch Studierende empfanden einen erhöhten Workload in den Corona-Semestern. Hierzu trug zum einen das neue Lehr-/Lernsetting bei, zum anderen war der fehlende physische Austausch während und außerhalb der Veranstaltungen dafür verantwortlich. Das Herstellen von Videos bzw. das mehrfache Anschauen dieser erhöhte deutlich den Workload für alle Akteurinnen. Für die Studierenden fiel das Peer-to-Peer-Lernen fast gänzlich weg und die Organisation des Studiums war herausfordernder als vor Corona. Für die Lehrenden war der Datenschutz in Bezug auf die Aufzeichnungen und das Anschalten der Kameras ein großes Thema. Dieses wurde dahingegen bei den Studierenden nur peripher angesprochen. Beide Akteur*innengruppen wiesen darauf hin, dass die Lehrenden als Alleinunterhalter die digitale Lehre technisch bewerkstelligen und die Studierenden zur Interaktion bewegen mussten, während diese eher zu Einzelkämpfern wurden und mit der Monotonie der digitalen Lehre und der Selbststrukturierung umgehen mussten. In den synchronen Veranstaltungen fand wenig Austausch statt. Kleine Gruppen und das Anschalten der Kameras unterstützten jedoch die Interaktion, darin waren sich Studierende und Lehrende einig. Für alle Beteiligten stellten der durch die digitalen Formate veränderte Lehr-/Lernraum und der ungewohnte, zum Teil sehr reduzierte Austausch einen zentralen Diskussionspunkt in den Fokusgruppen dar. 


\subsection{Gesamtsituation}

In diesem Kapitel wird herausgearbeitet, wie sich für Lehrende und Studierende die Lebensbereiche innerhalb und außerhalb der Hochschule unter den Pandemie-Bedingungen verändert haben. Hier wird der Fokus auf die Lebenssituationen und Herausforderungen, die damit einhergingen, gelegt. Dazu zählen unter anderem der Lehr-/Lernort, die Wohnsituation und der Ausgleich zum Beruf bzw. Studium.

\section{a. Perspektive der Lehrenden}

Also ich sitze zumeist in einem relativ kleinen und gemütlichen Arbeitszimmer, in dem ich eben wie gesagt mit zwei Rechnern, weil die immer nur wechselseitig funktionieren, leider deswegen, ((lacht)) ausgestattet sitze. (FGL1: 12)

Eine Veränderung der räumlichen Arbeitssituation kennzeichnet die Gesamtsituation der Lehrenden, die sowohl private als auch berufliche Lebensbereiche umfasst. Fanden in der Präsenzlehre die Vorlesungen in den Hörsälen oder in den Seminarräumen der Hochschulen statt, so mussten die Lehrenden zu Beginn der Corona-Semester ad hoc von der Hochschule ins Homeoffice als Arbeits- und Lehrort wechseln. Hierfür waren auch die entsprechende Einrichtung und Optimierung des jeweiligen Arbeitsplatzes notwendig, da idealerweise für die Lehre mindestens zwei Bildschirme notwendig waren. Headset, Mikrofon, Tablet mit Stift, entsprechende Ausleuchtung und Installation weiterer Programme waren aus Sicht der Lehrenden von Vorteil. Um Ruhe zu finden und ungestört arbeiten zu können, nutzten viele die Möglichkeit, sich in ihr Arbeitszimmer zurückzuziehen. Manche zogen in den Keller oder ins Kinderzimmer, für das Homeoffice wurden flexible Plätze im Haus belegt, weil einige Räumlichkeiten durch andere Familienmitglieder bereits genutzt wurden oder die Internetverbindung nur in bestimmten Räumen stabil genug war. Das Pendeln zwischen Hochschulbüro und Homeoffice stellte des Öfteren eine Alternative dar, um weiterhin eine wohltuende Abwechslung zu erfahren oder weil die Bandbreite zu Hause wegen der sich ebenfalls im Homeoffice befindlichen Familie nicht ausreichte. 
Also mein Lehrort sieht eigentlich normalerweise nicht so aus wie hier, sondern ich spüre das Corona nicht als Entlastung des Weges zur Hochschule, weil ich immer schaue, dass ich alle Vorlesungen von meinem Büro aus mache. Also der Unterschied ist Büro statt Hörsaal. Und da ist einfach mehr Power im Netz wie daheim. Ich habe zwar versucht, das aufzurüsten. Ich habe auch zwei Kinder, die gerade jetzt noch Homeschooling parallel haben. Das geht gar nicht, sage ich mal. Vor allem finde ich es auch hinreichend unprofessionell, wenn man vor hundert Leuten vorträgt, und die Leitung funktioniert nicht. (FGL2: 21)

Die Lehrenden sahen sich insgesamt dennoch in einer privilegierten Lebenssituation, da sie meist genügend Platz hatten und über mehr Möglichkeiten des Raumwechsels als die Studierenden verfügten.

Die Situation im Hometeaching war für die Lehrenden insofern neu, dass sowohl die Lehre als auch alle weiteren Arbeitstätigkeiten nun von zu Hause aus erledigt werden mussten. Vor Corona fand die Lehre im Hörsaal, im Seminarraum oder in Laboren statt, Besprechungen oder Gremiensitzungen wurden in Besprechungsräumen abgehalten. Für die Vor- und Nachbereitung der Lehre sowie Gespräche nutzten die Lehrenden ihr Arbeitsbüro an der Hochschule, während Lese- oder Vorbereitungstätigkeiten in weiten Teilen zu Hause stattfanden. Alle Tätigkeiten verlagerten sich nun in den Corona-Semestern an einen einzigen Ort: den eigenen Schreibtisch mit Bildschirm und Computer. Dadurch fand ein viel nahtloserer Übergang zwischen Lehrveranstaltungen, Studierendenbetreuung, Gremiensitzung und Korrekturen statt.

Diese neue räumliche Arbeitssituation hatte Konsequenzen für die Tagesstruktur und das Wohlbefinden der Lehrenden. Selbstverständliche Unterbrechungen oder Auflockerungen in der Präsenzlehre und die verschiedenen Wege - darunter der Weg zu anderen Kolleg*innen, der Raumwechsel, der Weg zur Kaffeeküche oder der Weg zu einem anderen Gebäude zwischen den Lehrveranstaltungen - fielen weg. Damit einhergehend berichtete ein Teil der Lehrenden von gesundheitlichen Beschwerden. Durch das viele Sitzen kam es zu Rückenschmerzen oder es musste eine neue Brille gekauft werden. Die Lehrenden beschrieben den Zustand als eine Art digitale Erschöpfung durch das pausenlose Arbeiten am Computerarbeitsplatz. Die permanente Nutzung digitaler Geräte war weitaus anstrengender als die Lehre in 
Präsenz, die Erschöpfung zeigte sich zudem mehr geistig als körperlich, so wie hier beschrieben.

Aber nach dieser ersten Phase kam dann wie gesagt auch die Phase der Ernüchterung. Und ich will es wirklich ehrlich sagen: Und am Ende des letzten Semesters auch der Erschöpfung. Also ich würde von so einer digitalen Erschöpfung durchaus reden, die damit einhergeht. Und das ist eigentlich erstaunlich, weil die körperlich ist man ja eigentlich weniger gefordert, als wenn man jetzt vor einem Publikum steht, (I: Ja.) im Klassenraum und sich bewegt usw. Und wo man denken müsste, eigentlich wäre das erschöpfender. Aber es ist eine, eine geistige Erschöpfung. (FGL1: 23)

In den Fokusgruppen tauschten sich Lehrende ebenfalls darüber aus, wie sie in den Corona-Semestern einen Ausgleich zur Berufstätigkeit fanden. Mehrheitlich wurde angegeben, in die Natur zu gehen, sich sportlich zu betätigen, sich auch virtuell mit Freunden zu treffen. Andere Ausgleichs- und Inspirationsmöglichkeiten wie Kultur, Museen und das Reisen waren nicht mehr möglich.

Durch die Verlagerung des Arbeitsplatzes ins Private veränderte sich auch die Struktur von Berufstätigkeit und privater Situation, es fand keine klare Trennung von Privatem und Beruf statt. Bisher bildete die räumliche Trennung automatisch eine Grenze, nun floss vieles ineinander, da die Berufstätigkeit in den privaten Räumen stattfand - jederzeit konnte der kleine Sohn zur Tür reinkommen. Durch die digitale Kommunikation per E-Mail, Chats und Messenger-Dienste entstand zudem ein Druck, immer erreichbar zu sein und schnell reagieren zu müssen.

Die sich dann halt auch ganz gnadenlos da eine Weiterleitung vom Telefon auf ihr Privathandy geschaltet haben und sich da auch quasi mehr oder weniger rund um die Uhr anrufen lassen und so. Und das mache ich persönlich zum Beispiel nicht, aber das schafft natürlich sehr ungleiche Erwartungshaltungen auch, ne? ((Herr B nickt)) Also es gibt dann halt irgendwie Leute, die sagen so: „Wie, du hast keine Weiterleitung geschaltet?" (FGL6: 65)

Aus Sicht der Lehrenden bildete der übergangslose Rollenwechsel eine weitere Herausforderung: Kaum war der Computer ausgeschaltet oder sie verließen das Arbeitszimmer, waren sie wieder Eltern. Die Zimmertür und der Computerknopf leiteten unmittelbar in eine andere Rolle über. 
Ich denke, das ist auch ja inzwischen, sagen wir mal, das ist ja fast banal: Dieser nicht vorhandene Zeitraum zwischen Rollenwechseln, das ist eigentlich das, was mir dabei am schwersten fällt. Also ich klappe den Rechner und ich hab' ihn noch nicht zu, da bin ich Mutter. Und das hört man ja überall, ne? Dass dieses sozusagen übergangslose Von-einer-Rollein-die-andere-Gleiten, haben ist aus meiner persönlichen Perspektive die größte Herausforderung. (FGL4: 16)

Die befragten Lehrenden waren sich darin einig, dass sich die Hometeaching-Situation insbesondere für Lehrende als herausfordernd darstellte, die mit Kindern in einem gemeinsamen Haushalt leben. Durch die Corona-Pandemie wurde mehr gekocht und Lernstoff erklärt, die Kinder brauchten Beschäftigung, da Schulen und Kinderbetreuungseinrichtungen weitestgehend geschlossen waren, außerdem fanden die organisierten Freizeitaktivitäten in Vereinen oder Gruppen nicht statt. Ferner fehlten den Kindern ihre Freunde, wodurch sie den Eltern mehr Aufmerksamkeit abverlangten. Nicht nur die Kinder, sondern auch die Partner waren plötzlich wieder größtenteils im Haus. Das komplette Leben verlagerte sich zurück in die eigenen vier Wände. So kam aufgrund von Aufgaben und Verpflichtungen im familiären Umfeld zusätzlicher Zeitdruck auf.

Sie sehen, hinter mir turnt mein noch nicht ganz zweijähriger Sohn rum. Das heißt, das Sommersemester, da habe ich nachts gearbeitet. Jetzt im Wintersemester war es etwas besser, da konnten wir die Notbetreuung nutzen. Und es war ja zum Teil auch noch nicht ganz gelockdowned. Also mein Privatleben war inexistent. Der Tag begann um 8 Uhr, um 18 Uhr wurde ich abgelöst in der Kinderbetreuung, und dann begann ein Arbeitstag. Also beschissen einfach, muss man einfach sagen. (FGL1: 68)

Die zeitliche Flexibilität durch die digitale Lehre kam den Lehrenden zugute, um erfolgreich zwischen den verschiedenen Rollen zu jonglieren. Betreuungsgespräche mit Studierenden konnten am Abend stattfinden, das Lehrvideo wurde in den Abendstunden oder am Wochenende gedreht, wenn die Partner*innen sich um die Kinder kümmern konnten.

Also es ist durchaus so mit meinem Sohn, dass er sehr viel zu Hause ist. Jetzt gerade ist er im letzten Jahr in die Schule gekommen und das ist ja auch so ein bisschen schwierig. Da versuche ich eben zusammen mit meiner Frau entsprechend zu jonglieren, wer sich jetzt gerade um den Nachwuchs kümmern kann und wer nicht. Ich für meinen Teil war dann tatsächlich auch sehr froh, dass ich eben diesen Weg gewählt habe 
mit diesen Lehrvideos, weil ich da für mich selber, also nicht nur die Studierenden eine zeitliche Flexibilität haben, sondern ich selber eben auch eine zeitliche Flexibilität. (FGL6: 69)

Dagegen erlebten die Lehrenden ohne Kinder oder mit erwachsenen Kindern die Homeoffice-Situation als angenehmer, da ein konzentriertes Arbeiten und mehr Flexibilität in der Tagesstruktur möglich waren und in der Mittagspause auch Zeit für Joggen oder Spazierengehen war.

\section{b. Perspektive der Studierenden}

Oh, wie gerne würde ich jetzt rausgehen und mit meinen Kommilitonen draußen stehen, kurz in die Mensa rennen, mir einen Kaffee holen, der einfach nicht schmeckt ((Frau G lacht)) und überteuert ist. Ja, nee, aber ich sage das deswegen so bewusst, weil das ist das, was fehlt. (FGS3: 67)

Vor allem das Fehlen des physischen Austauschs war für die Situation der Studierenden in den Corona-Semestern kennzeichnend. Der Weg zur Hochschule und die Hochschule als Lernort und Treffpunkt fielen weg. Die Studierenden waren komplett auf ihre Wohnräume zurückgeworfen. Die Gesamtsituation der Studierenden ist, wie bei den Lehrenden, durch eine räumliche Verschiebung gekennzeichnet.

In den Corona-Semestern war der komplette Umzug zurück ins Elternhaus und dementsprechend ins Kinderzimmer aufgrund der finanziellen Situation oft notwendig: „Jetzt bin ich seit ein paar Tagen wieder bei meinen Eltern zu Hause, weil es mit der Miete usw. finanziell nicht mehr gereicht hat" (FGS6: 49). Dahingegen verbesserten einige Studierende ihre Wohnsituation, vom Wohnheim in eine WG, von einer WG ohne Wohnzimmer in eine WG mit Wohnzimmer oder direkt in eine eigene Wohnung: „Dementsprechend habe ich mir jetzt zum Wintersemester wieder eine eigene Wohnung geholt und habe jetzt mir ein eigenes Büro eingerichtet“ (FGS5: 12). Vereinzelt wurde erwähnt, dass sich die Wohnsituation während der Corona-Semester nicht änderte.

Die veränderte Lernsituation wurde mehrheitlich als sehr herausfordernd wahrgenommen, denn Wohn-, Studien- und Lernort war nun identisch. Die Trennung zwischen Lern- und Lebensort fehlte und erschwerte das Homestudying und damit die Gesamtsituation deutlich. Der Lernort in der eigenen Wohnung war vermehrt der Schreib- 
tisch, dennoch wurden auch andere Räume zum Lernen genutzt, beispielsweise das Wohnzimmer, der Küchentisch oder das Gästezimmer, vereinzelt das Sofa oder gar das Bett. Das eigene Büro, der Garten und die Labore der Hochschule, die die Studierenden teilweise nutzen konnten, wurden ebenfalls punktuell als Lernort genannt. Vor allem die Bibliothek und das gesamte Hochschulgelände fehlten den Studierenden als Lernort. So war es in den Corona-Semestern nicht mehr möglich, einfach auf dem Campus zu lernen.

Wir durften uns auch gar nicht auf dem Campus aufhalten, ohne da nachzuweisen, dass wir hier einen Termin haben oder so, also auch da wieder, wenn wir Abstand gehalten hätten oder irgendwas, ja, also es war einfach verboten. Das heißt, uns wurde eigentlich so jede Lernmöglichkeit genommen im Prinzip. (FGS3: 63)

Insgesamt wurde das digitale Studium durch mangelnde technische Ausstattung kaum beeinträchtigt. Auch waren die befragten Studierenden mehrheitlich zufrieden mit ihrer technischen Ausstattung und kamen mit der jeweiligen Hard- sowie Software gut zurecht. Die Ausstattung reichte von einem Laptop bis zu einem PC mit mehreren Bildschirmen und externem Mikrofon. Fallweise wurden zusätzliche Geräte angeschafft oder vorhandene durch höherwertige ersetzt, auch die Internetleistung wurde verbessert. Das Mittippen während der synchronen Lehrveranstaltungen, wenn nur ein Laptop vorhanden war, wurde als herausfordernd beschrieben. Die Videokonferenzsysteme und Austauschplattformen, die für die digitale Lehre genutzt wurden, führten ebenso zu Schwierigkeiten. Es wurde unterdies berichtet, dass Kompatibilitätsprobleme entstanden, zu viele verschiedene digitale Werkzeuge von den unterschiedlichen Lehrenden benutzt wurden oder Hilfestellungen vonseiten des Rechenzentrums fehlten.

Die finanzielle Situation ermöglichte es nicht, den eigenen Lernplatz lernoptimal einzurichten, wodurch das Lernen ebenfalls erschwert wurde:

Ich meine, wir Studenten ((lacht)) sind ja jetzt nicht bekannt dafür ((Frau U lacht)) dass wir uns total viel leisten können. Wenn wir jetzt sagen, hey, ich brauche zwei neue Bildschirme oder so, damit ich einen richtigen Arbeitsplatz habe, am besten noch einen ergonomischen Schreibtisch und alles, dass ich wirklich gut arbeiten kann, das ist halt nicht für jeden vielleicht so möglich. (FGS4: 81) 
Durch die fehlenden Freizeitaktivitäten gingen die Ausgaben zwar zurück, jedoch wurde in höherwertige Lebensmittel investiert, zumal Kochen und Essen oftmals zum Highlight des Tages geworden war: „Und deswegen gönnt man sich einfach was im Supermarkt, was man sonst halt eher nicht macht, kauft vielleicht was Besseres, was Teureres und damit also: ((lacht)) Ja, Kochen und Essen ist dann die Hauptausgabequelle“ (FGS2: 119).

Die finanzielle Situation war grundsätzlich durch die Unterstützung der Eltern, Werkstudenten- bzw. Nebentätigkeiten, Gründung und Berufstätigkeiten oder BAföG, Kredit und Unterstützungshilfen gesichert. In den Fokusgruppen wurde auch von Kommilitoninnen berichtet, die aufgrund der gravierenden finanziellen Situation das Studium abbrechen oder von Lebensmittelspenden leben mussten:

Ich habe auch eine Kommilitonin, die holt sich Lebensmittelspenden also aus einem Regal, was halt in der Stadt daliegt, weil, da gibt es einfach ein Regal, wo Lebensmittel abgelegt werden. Und wenn es da nichts gibt, dann gibt es nichts zu essen. (FGS3: 91)

Es wurde außerdem ausführlich darüber berichtet, worin Ausgaben und Einnahmen bestanden. Nicht nur die Lebensmittel wurden höherwertig und teurer, sondern auch das permanente Zuhause-Sein führte zu mehr Nebenkosten. Onlineshopping oder Technikanschaffungen wurden als weitere Ausgaben genannt. Auf der anderen Seite fielen Ausgaben durch die Einschränkung der Freizeitaktivitäten und Fahrtkosten weg. Vereinzelt gaben Studierende auch an, dass ihre Einnahmen durch Gründung oder den Wechsel von Nebentätigkeit zu Werkstudententätigkeit in den Corona-Semestern gestiegen waren.

Die digitale Lehre und das digitale Lernen führten dazu, dass die gewohnte Tagesstruktur nicht mehr gegeben war. Die Struktur des Studiums wurde wie zuvor durch einen Stundenplan gegeben, durch Aufzeichnungen der Veranstaltungen oder Videos war aber die aktive Teilnahme nicht mehr notwendig. Das Studium musste folglich aktiv gestaltet werden. Teilweise wurden hierfür selbst Wochenpläne angefertigt, die den Stundenplan, sportliche Aktivitäten und Zeiten zum Kochen und Essen beinhalteten. Durch das permanente Sitzen vor dem Bildschirm beschrieben die Studierenden eine Laptopflucht: Digitale Treffen mit Freund*innen wurden teilweise nicht mehr wahr- 
genommen, weil der Tag bereits gänzlich in dem Sitzen vor dem Bildschirm bestand. Die digitale Erschöpfung zeigte sich auch in einem Schwinden der Motivation, einer kürzeren Aufmerksamkeitsspanne und einem Gefühl, dass das digitale Lernen anstrengender war. Daraus resultierte, dass das Wohlbefinden der Studierenden schwand. Es wurde des Weiteren eine Verschlechterung der Gesamtsituation im Laufe der Corona-Semester beschrieben.

Ja, der Kontakt mit den Kommilitonen ist weg, dass jegliche Bars oder jegliche Möglichkeit, irgendwas in der Freizeit zu machen, sei es im Fitnessstudio, sei es ein Kino, sei es ein Hallenbad oder was ganz Banales, einfach nur eine Laufbahn, ja. Ist alles weggefallen, alles ist zu. Und ja, man verbringt eigentlich die meiste Zeit nur noch vor dem Laptop und hat eigentlich keine Lust mehr, sich diesen Kasten anzuschauen ((alle lachen)), aber ist leider gezwungen, sich das dann jeden Tag doch noch mal länger anschauen zu müssen. (FGS1: 96)

Die Umstellung auf die digitale Lehre war für erfahrene Studierende herausfordernd, da ihnen das System der Hochschule vertraut war und die funktionierende Lernstrategie verändert werden musste. Einige fühlten sich wieder wie Erstsemester. Diese herausfordernde Situation wurde durch gesundheitliche Probleme verstärkt, die mit der erhöhten Bildschirmzeit und dem Bewegungsmangel einhergingen. Von Rückenund Augenproblemen war dabei vermehrt die Rede.

Es ist ja nicht zu Ende, wenn man dann seine Vorlesung beendet hat, sondern die Arbeit, also gerade für uns geht die Arbeit ja am PC weiter. ((Frau V nickt)) Und es war dann schon sehr schwierig auch/ ich würde sagen, auch gesundheitlich, dass man viel Kopfschmerzen hat. Oder meine Augen haben sich auch total verschlechtert. (FGS4: 13)

Auch die psychische Belastung nahm zu: „Eigentlich nicht nur die Motivation für das Studium, sondern eigentlich schon fast die Psyche, die da dann irgendwann mal einen Knacks gekriegt hat" (FGS1: 101). Um einen Ausgleich zum Studium zu gewährleisten, trafen sich Studierende per Videokonferenz mit Kommilitoninnen und Freund ${ }^{\star}$ innen. Der Freundeskreis aus der Heimat wurde oft zu einem lokalen „,vom Zimmer aus erreichbaren“ (FGS1: 130) Freundeskreis. Hiermit gingen jedoch auch Konflikte mit Mitbewohner*innen einher, auf die in den Fokusgruppen nicht näher eingegangen wurde. Der Ausgleich wurde folgendermaßen beschrieben: „Genauso sind wir aber auch irgendwie 
im Freundeskreis ziemliche Spaziergänger geworden“ (FGS1: 129). Die Freizeitaktivitäten verschoben sich ins Digitale in Form von Zocken, Fernsehen oder digitalen Treffen oder wurden allein bzw. zu zweit praktiziert, z.B. durch Yoga, Lesen oder Spazierengehen. Durch den Wegfall von Feiern hatten die Studierenden zwar weniger Ausgaben, aber es entstanden Verlustgefühle. Dabei wurde vor allem das Feiern der vollendeten Prüfungsphase deutlich als Verlust gekennzeichnet.

Aber die Motivation war echt weniger. Und ich habe auch mit anderen darüber gesprochen und ich glaube, ein Punkt ist auch, dass man nicht so was hat, worauf man sich danach freut. Das klingt doof, ((alle lachen)) aber normal war halt Prüfungsphase und danach feiert man oder geht reisen oder hat irgendwas vor. Und jetzt ist halt so: Ja, wieder vorbei. (FGS2: 32)

Hier wurde ein Verlust von Motivation und Spaß am Studium deutlich. Das wurde auch in einer weiteren Fokusgruppe betont: „Also meine Meinung: Es macht keinen Spaß, aber es funktioniert“ (FGS5: 121). Die Begründung hierfür lautete, dass „dieses Konstrukt Studium ja noch viel, viel größer ist und viel, viel mehr beinhaltet als eben nur, ja, das Lernen“" (FGS3: 68). Das gesamte Studierendenleben, d.h. all dies, was Studierende außerhalb des Studiums unternehmen, fehlte in den Corona-Semestern. Darüber hinaus führte die HomestudyingSituation dazu, dass sich Studierende Gedanken über den Studienverlauf und das allgemeine Gelingen ihres Studiums machten, zumal Auslandspraktika und andere studienaffine Tätigkeiten nicht möglich waren. Aus der fehlenden Trennung zwischen Privatem und Studium entwickelte sich häufig ein schlechtes Gewissen, wenn sich die Studierenden um einen Ausgleich in der Freizeit kümmerten. Grundsätzlich nahm das Studium in den letzten beiden Corona-Semestern einen deutlich größeren Raum im Leben der Studierenden ein.

\section{Zusammenführung}

Insgesamt wurde deutlich, dass die Lebensbereiche innerhalb und außerhalb der Hochschule insbesondere durch die fehlende Trennung zwischen Beruf bzw. Studium und Privatem gekennzeichnet waren. Homestudying und Hometeaching wurden von beiden Akteur*innen- 
gruppen jeweils als herausfordernd wahrgenommen. Folgende Aspekte tauchen dabei beidseitig immer wieder auf (s. Abbildung 16).

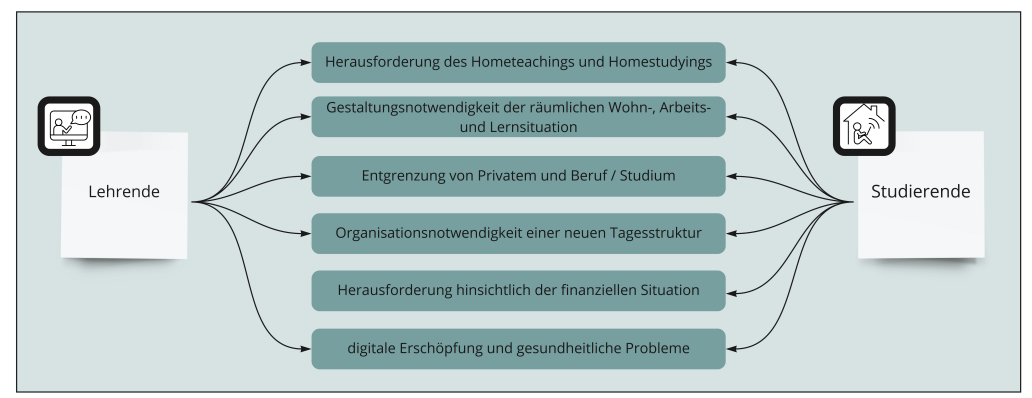

Abbildung 16: Aspekte Hometeaching- und Homestudying-Situation

Zusammenführend zeigt die Gesamtsituation, dass sowohl Studierende als auch Lehrende mit den Umständen zwar zurechtkamen, aber darin deutliche Herausforderungen erlebten. Wohn- und Lehr-/Lernort wurden durch die Corona-Pandemie zu einem Ort. Die Wohnsituation der befragten Lehrenden war dabei insgesamt entspannter als die der Studierenden, wenn ein Ortswechsel im eigenen Haus bzw. der eigenen Wohnung oder in das Hochschulbüro noch möglich war. Bei den Lehrenden kam die fehlende Rollentrennung hinzu, zum Beispiel durch Betreuung der Kinder. Bei den befragten Studierenden war die fehlende Trennung zwischen Privatem und Studium dadurch deutlich spürbar, dass ein Ortswechsel kaum möglich war. Die finanzielle Situation kam bei den Lehrenden nur dadurch zur Sprache, dass sie selbst ihre Lage im gesamtgesellschaftlichen Vergleich als privilegiert beschrieben. Von der überwiegenden Zahl der befragten Studierenden wurde die finanzielle Situation als gut bzw. ausreichend bezeichnet. Vereinzelt wurden finanzielle Schwierigkeiten angerissen. Durch die Reduktion von Lehren und Lernen auf den heimischen Computerarbeitsplatz war in beiden Gruppen zudem eine deutliche digitale Erschöpfung wahrzunehmen. In beiden Gruppen wurde versucht, diese mit Aktivitäten auszugleichen. Sport, Spazierengehen und ähnliche Einzelaktivitäten waren oft der einzige Ausgleich. Freund ${ }^{\star}$ innen wur- 
den in beiden Gruppen eher im digitalen Raum getroffen, was mitunter die digitale Erschöpfung verstärkte.

\subsection{Blick in die Zukunft}

In diesem Unterkapitel werden die Vorteile, Chancen und Wünsche der befragten Lehrenden und Studierenden beschrieben. Darüber hinaus wurden von den Teilnehmenden selbst Handlungsempfehlungen für Studium und Lehre unter Corona-Bedingungen sowie Post-CoronaZeit formuliert.

\subsubsection{Didaktische und technische Ebene}

\section{a. Perspektive der Lehrenden}

Ich wurde zum Digital Native ((alle lachen)). (FGL8: 12)

Es bestand bei den Befragten Einigkeit darüber, dass der Lernfortschritt enorm war, da kaum Vorerfahrungen bei den Lehrenden vorhanden waren. Darüber hinaus war der „Methodenblumenstrauß größer geworden ... und man kann aus einem viel größeren Repertoire wählen, als man das im Vorfeld hatte" (FGL6: 71). Es entstand laut den interviewten Lehrenden ein Raum, in dem didaktisch experimentiert werden konnte. Die Analyse des Materials zeigte, dass ein gegenseitiges Verständnis vorhanden war, da im Zuge der Ad-hoc-Umstellung die Umsetzung noch nicht ideal funktionierte.

Also wir haben jetzt sehr viel gelernt, Sie haben es eben gesagt, Herr C, dass in den ersten drei, vier Wochen das dann eine sehr schnelle Lernkurve war. Und ich glaube, das hat auch damit zu tun, dass jeder im Wasser war und schwimmen lernen musste und dass es halt auch irgendwie toleriert war, dass da Dinge mal nicht so gut funktionieren auf Anhieb. Und dieses Experimentieren, das würde ich mir wünschen, dass wir das irgendwie beibehalten können, zumindest eine Zeitlang. (FGL6: 85)

Es wurde zudem von den Lehrenden als positiv eingeschätzt, dass Studierende Hilfe anboten und Tipps oder Verbesserungsvorschläge bezüglich der Nutzung digitaler Werkzeuge gaben. „Also dass man das 
Gefühl hatte, keine Ahnung, ich weiß, ich habe mal irgendwann ein Video teilen wollen und habe vergessen, den Ton zu aktivieren, dass man dann sich so gegenseitig hilft" (FGL1: 20).

Digitale Formate schufen räumliche und zeitliche Flexibilität. Es konnte festgestellt werden, dass aufgrund der digitalen Lehre externe Expert ${ }^{*}$ innen an Veranstaltungen teilnehmen konnten, da der Reiseaufwand wegfiel. Einige Lehrende wünschten sich, dass ein Teil der Vorlesungen, Besprechungen oder Gremiensitzungen in Zukunft digital stattfänden. Dies vermeide auch den Ausfall von Vorlesungen beispielsweise bei Dienstreisen: „Ich halte die Vorlesung, die ich 9:45 Uhr vormittags nicht halten konnte, vielleicht $18 \mathrm{Uhr}$ abends digital und habe dann keinen Wegfall" (FGL1: 98). Laut den Lehrenden konnte festgestellt werden, dass sich digital die Betreuung von Abschlussoder Praxisarbeiten bei räumlicher Distanz viel besser organisieren ließ. „Viele Dinge, die bisher eine Tagesreise waren, sind in Zukunft eine Konferenz von einer Stunde via was auch immer, BigBlueButton, WebEx" (FGL6: 71). Auch für die Studierenden bot die räumliche Flexibilität Vorteile, denn sie konnten an Fachkonferenzen teilnehmen, da sie nicht auf eigene Kosten anreisen mussten. Auch Exkursionen wurden im digitalen Format getestet.

Die Exkursion ist dementsprechend digital gewesen und es waren insgesamt über 250 Teilnehmer mit dabei. Das sind Vorteile, finde ich. Erst recht: Man kann die Leute wirklich direkt an die Altanlagen führen, man und da stehen dann 250 Leute in der ersten Reihe und nicht irgendwie noch hintendran. (FGL5: 49)

Die Vielfalt der Lehrformate schuf viele neue Möglichkeiten, die die Lehrenden auch zukünftig nutzen möchten. Aufgrund der Mischung von synchronen und asynchronen Elementen bestand die Möglichkeit, die Lehre abwechslungsreicher gestalten, sodass Ermüdung möglichst vorgebeugt wurde. Die asynchronen Elemente wie Videoaufzeichnungen, Übungen oder Tests boten den Studierenden die Möglichkeit, mehr in ihrem eigenen Lerntempo zu lernen. Die Modelle des Inverted Classrooms zeigten, dass eine Vorbereitung der digitalen Veranstaltung mit Videos den synchronen Austausch erleichterte. Gruppenarbeiten wurden über Breakout Rooms in kurzer Zeit mit geringem Aufwand umgesetzt. Manche Lehrenden nutzten zusätzlich virtuelle Whiteboards, die ihnen zufolge eine gute Alternative darstellten und 
somit Moderationskoffer und Flipcharts ersetzten. In den Interviews wurde von den Lehrenden betont, dass sie insbesondere auf den Chat als Kommunikationskanal nicht mehr verzichten wollten. Es zeigte sich, dass Studierende sich eher trauten, im Chat eine Frage zu stellen, als sich in der Veranstaltung zu Wort zu melden: „Diese Chatfunktion in dem MS Teams. Das hat bei mir extrem gut funktioniert, dass sich die Studenten da an mich gewendet haben und auch relativ mit einer relativ geringen Hemmschwelle“ (FGL1: 46).

Ausführlich diskutiert wurde in den Fokusgruppen der Lehrenden, inwiefern manche Lehrinhalte in einem digitalen Format besser als in einem Präsenzformat zu unterrichten sind - hier wurden die Fächer genannt, bei denen es um die reine Vermittlung von Grundlagenwissen ging. Wichtig war den Lehrenden dabei, dass nicht alle Formate digital umsetzbar sind. Die Lehrenden sahen die Notwendigkeit von Präsenz insbesondere in den naturwissenschaftlichen und technischen Fächern. Vor allem beim Lernen an Maschinen, in Laboren sowie bei Experimenten müssten die Studierenden selbst an den Gerätschaften arbeiten.

Das ist ganz wichtig und auch die Umsetzung, also das Handwerkliche. In der Chemie muss man eine Glasapparatur aufgebaut haben. ((Frau Q nickt)) Oder man lernt auch nicht Schweißen durch ein YouTube-Video ((Frau Q nickt)), ((Herr S lacht)), sondern das Handwerkliche muss man einen Lack mal in der Hand gehabt haben, das ist extrem wichtig. (FGL3: 104)

Andere Lehrende berichteten, dass digitale Formate, die beispielsweise im Bereich Design-Industrie bereits Verwendung finden, übernommen wurden. Entwürfe konnten digital besprochen werden und der Lehrende konnte direkt mit seinem digitalen Stift in die Entwürfe zeichnen, ohne das Original zu verändern.

Die Lehrenden formulierten, dass in Zukunft ggf. hochschulübergreifend gearbeitet werden kann. Zum Beispiel könnte das Hochschulnetzwerk Digitalisierung besser genutzt werden, indem perspektivisch ein hochschulübergreifender Austausch von Lehrmitteln oder die Integration von frei zugänglichen Lehrinhalten gefördert wird.

Zentrum für Open Educational Resources Baden-Württemberg. Und da finden Sie auch schon Vorlesungen von Kollegen. Und das ist ein ganz 
zartes Pflänzchen. Und man kann nur hoffen, dass das irgendwo weitergetrieben wird. (FGL5: 88)

Des Weiteren wurde von den Lehrenden gefordert, dass eine hochschulspezifische Ausrichtung nicht verloren gehen sollte. Die HAWs fokussierten zudem die Themen der Future Skills und der digitalen Kompetenzen, um Studierende auf die Arbeitswelt vorzubereiten. Daher braucht es, laut den Befragten, digitale Elemente im Studium. Dieser Aspekt führte zu Fragen nach der Art und Weise des Lernens und welche Räumlichkeiten zukünftig benötigt werden.

Meine Vorstellung ist: Ich glaube, wir werden weggehen von den großen Hörsälen, die ja eh nicht riesig sind an unserer Hochschule, aber zu viel mehr Gruppenarbeitsräumen, zu viel mehr Kleingruppenarbeit, zu viel mehr aktiven, gut gestellten Fragen, die selbst über Denken beantwortet werden, wo selber Recherche stattfindet. Wir werden aus meiner Sicht $\mathrm{zu}$ Lernbegleitern werden und nicht zu Inputgebern oder jedenfalls nicht hauptsächlich. Da werden Inputs gesetzt und dann Lernbegleitung stattfinden. Das wäre meine Vorstellung, aber vor allem auch mein Wunsch. (FGL1: 102)

Die Analyse des Materials deutete darauf hin, dass es eines didaktischen Diskurses über die Post-Corona-Lehr-/Lernkultur bedarf. Dieser Diskurs beinhaltet darüber hinaus die Aufgaben und die Rolle der Lehrenden: „Also wir bereiten ja Studierende auf eine sehr ungewisse Zukunft vor. Und da werden sie ganz andere Kompetenzen dafür brauchen, als wir teilweise auch heute ihnen vermitteln" (FGL1: 82). Die Lehrenden stellten sich diesbezüglich die Frage, über welche Fähigkeiten die Studierenden am Ende des Studiums verfügen müssen. „Was macht denn wirklich Hochschullehre aus? Das hoffe ich, dass das quasi mitgenommen wird, dass hier weiterdiskutiert wird und auch im Kontext mit digitalen Medien“" (FGL2: 102). Die Corona-Semester waren laut den Lehrenden, neben den Herausforderungen, eine Chance, dass Studierende mehr Eigenverantwortung übernahmen und aktiv ihr Lernen organisierten: „Auch in diese eigenverantwortliche Rolle reinwachsen. Was ich wirklich überhaupt nicht leiden kann, ((lacht)) das ist so dieses Thema: Ich sitze als Studierender da und konsumiere. Also nach dem Motto: Dozent, jetzt liefere mal irgendwie eine gescheite Vorstellung hier. Ja?" (FGL2: 90). 
Chancen für die Post-Corona-Lehre sahen die Lehrenden auch in der Möglichkeit, Onlineprüfungen anzubieten, wodurch eine größere Vielfalt an Prüfungsformaten, Zeitvorteilen und mehr Flexibilität entstehen könnten. Eine wichtige Voraussetzung war für die Lehrenden der professionelle Einsatz von digitalen Formaten, wofür weiterer Support sowohl für Lehrende als auch Studierende als notwendig erachtet wurde, damit ein entsprechender Standard erreicht werden kann.

Also ich wünsche mir für eine Post-Corona-Zeit, dass der Umgang mit den digitalen Medien und der digitalen Lehre eine Selbstverständlichkeit wird und dass es nicht mehr darum geht, ob oder ob nicht, sondern höchstens, welche Instrumente man einsetzt, dass man verschiedene Formate etabliert Also hier muss man ja auch auf didaktische Dinge achten, dass einfach das Rüstzeug professioneller wird, die Hochschulen das zur Verfügung stellen, Schulungen, Weiterbildungen anbieten und die Studierenden im Rahmen von entsprechenden Vorlesungen und Coachings auch an das Thema heranführen. (FGL7: 111)

Grundlagenschulungen über das Drehen von Videos für Lehrende und Studierende wären eine mögliche Lehrveranstaltung in der PostCorona-Hochschullehre, so einige der befragten Lehrenden. Verbesserungsbedarf sahen die Lehrenden in der technischen Ausstattung: „Die Technik muss auf jeden Fall besser werden“ (FGL7: 86). Dies beinhaltete auch die weitere Verbesserung der Infrastruktur der Hochschule, die noch nicht abgeschlossen ist. Hier zeigten sich aus Sicht der Lehrenden auch Unterschiede zwischen den Hochschulen bzw. Studiengängen, die sich bereits mit Technik, digitalen Werkzeugen, Computerprogrammierungen und Ähnlichem auskannten.

Wo bitte, sind hier die Mittel des Ministeriums, die Förderung von Kompetenz, von digitaler Kompetenz, die wir an Studierende rankriegen, auch in Studiengängen, die das nicht wie Medienstudiengänge schon mal per se drin haben. (FGL4: 39)

In diesem Zusammenhang wurde der Wunsch der Lehrenden nach einer Vereinheitlichung der Strukturen und Standardtechnik in Bezug auf die Nutzung von Lernplattformen und Videokonferenzsystemen formuliert: „Also eine gute Standardtechnik, leistungsfähig und dann aber einfach noch so passende Tools, die man für den eigenen Bereich braucht. Ich glaube, das ist die Zukunft“ (FGL7: 86). 


\section{b. Perspektive der Studierenden}

Und man halt einfach in seinem Tempo machen kann. Und wenn man zum Beispiel jetzt ein Video-Podcast anhört, dann kann man einfach Stopp machen und genau. Also das sind, denke ich, so die Vorteile, dass man sich es einfach flexibler einteilen kann. (FGS2: 24)

Flexibilität in Zeit und Ort wurde, wie dieses Zitat zeigt, von den befragten Studierenden als Vorteil der digitalen Lehre beschrieben und auch als Chance für die zukünftige Lehre gesehen. Mit der zeitlichen Flexibilität geht der Nutzen der eigenen Lerngeschwindigkeit einher.

Bei einigen Studierenden kam durch die digitale Lehre die Ersparnis der Fahrtzeit hinzu, die das Lernen und das Leben entspannte: „In meinem speziellen Fall auch ziemlich Zeit gespart, weil ich da insgesamt drei Stunden Fahrtzeit pro Tag ((Herr B nickt)) nicht mehr brauchte, sondern halt innerhalb von ((lacht)) fünf Sekunden im Vorlesungssaal saß sozusagen“ (FGS6: 47). Die örtliche Flexibilität war vor allem im Zusammenhang mit Gruppenarbeiten wertvoll, denn dann konnte digital zusammengearbeitet werden, während $e^{*} e^{\star} r$ ggf. im Zug oder bei seinen/ihren Eltern war.

Elemente, die in der Post-Corona-Lehre beibehalten werden sollten, wurden viele genannt. So wollten die Studierenden bestenfalls alle Vorteile der digitalen Lehre behalten, aber auch zurück in die Präsenzlehre. In Theorie- und auch Mathematik-Vorlesungen waren die digitalen Veranstaltungen besser als vor Ort in der Hochschule, da sie prägnanter und kürzer waren, so die Studierenden. Zwei Studierende beschrieben es so: „Was meine [Hochschule] zum Beispiel auch beibehalten will, ist: Die Theorie-Vorlesungen, die müssen eigentlich einfach nicht in Präsenz stattfinden“ (FGS6: 117) und „die theoretischen Fächer klappen sehr gut, die klappen besser als in der Vorlesung vor Ort“ (FGS1: 22). Des Weiteren wurden Podcasts, Videos und Aufzeichnungen als wertvolle Elemente der zukünftigen Lehre genannt. WebEx, MS Teams, ILIAS und Discord wurden als digitale Werkzeuge, die vorteilhaft für das gesamte Studium sind, beschrieben.

Fragestunden und Diskussionsrunden, die zusätzlich zu Videos, Aufzeichnungen oder synchronen Veranstaltungen stattfanden, wurden ebenfalls als unterstützend für das Lernen wahrgenommen. Darüber hinaus wurde die Flexibilität der Sprechstunden wertgeschätzt, da die 
Studierenden nicht extra zur Hochschule mussten, um an Absprachen und Abschlussarbeitsbetreuungsgesprächen aktiv teilnehmen zu können.

Einige Lehrende waren durch die digitale Lehre besser erreichbar, konnten schneller auf die individuelle Situation der Studierenden reagieren. Positiv hervorgehoben wurde auch, dass weniger Veranstaltungen in den Corona-Semestern ausfielen als zuvor. Besonders motivierend und das Lernen unterstützend waren Lehrende, die sich auch in der synchronen Lehrsituation locker und lustig verhielten:

Aber gerade in dem einen Fach bzw. bei dem einen Dozenten war es aus irgendeinem Grund deutlich gelockerter. Also der hat auch sehr gerne über sich selber erzählt und was er macht und ... man hat auf jeden Fall gemerkt, dass halt generell man da als Studenten oder Studentin halt da auch irgendwie lockerer reingegangen ist. Und da wurde auch viel mehr erzählt, geschrieben im Chat und halt auch ... Sachen, die jetzt nicht wirklich zum Fach in dem Moment beigetragen haben, aber die halt trotzdem dann zu einer Diskussion oder zu einem Gespräch eingeladen haben, sei es jetzt um irgendein Spiel oder ein Video, irgendwas Bestimmtes. Und da war auf jeden Fall das deutlich lockerer, also das ging auch wirklich vom Dozenten aus. Die erste Vorlesung war quasi eine Stunde, wo er über sich selber erzählt hat ((Frau R, Frau S lachen)) und halt auch sich selber vielleicht nicht $\mathrm{zu}$ ernst genommen hat und da einfach mit einer Leichtigkeit rangegangen ist und das hat sich recht gut übertragen. (FGS7: 98)

\section{Insgesamt schuf die Vielfalt der Lehr-/Lernformate also neue Mög- lichkeiten und vereinfachte das Lernen.}

Aber ich kann es, also auf jeden Fall als Addition, als zusätzlich fand ich es sehr gut ((Frau R, Frau S, Frau T nicken)) einfach zum SelberWiederholen und wenn das Video dann halt eine Stunde ging, dann konnten manche Dozenten oder Dozentinnen mögen auch recht langsam sprechen, was ich auch eigentlich ganz gut finde, weil man es dann auf jeden Fall versteht. (FGS7: 109)

Aufzeichnungen von synchronen Veranstaltungen und Videos als Lernunterstützung wurden in allen Fokusgruppen mit Studierenden als Vorteil bezeichnet und damit einhergehend für die zukünftige Lehre als wertvoll wahrgenommen. Diese beizubehalten würde die Post-Corona-Lehre flexibler und damit einfacher für die Studierenden gestalten: „Das ist cool, dass das so flexibel ist und das würde ich mir wünschen, dass Aufzeichnungen irgendwie weiter online gestellt 
werden“" (FGS2: 147). Auch Breakout Rooms wurden positiv hervorgehoben, denn darin konnten kleine Gruppen mit angeschalteter Kamera und Mikrofon in einer aktiven Interaktion und Teilnahme lernen. Es zeigte sich diesbezüglich, dass bei ausgeschalteten Kameras eine passive Teilnahme und eine gewisse Anonymität vorherrschten. Daraus entstand der Wunsch, die Kameras bei digitalen Veranstaltungen in den nächsten Corona-Semestern anzuschalten. Inverted Classroom als Lehrformat wurde von den Studierenden auch als sehr vorteilhaft beschrieben:

Ich kam in den Genuss, Mathematik 2 quasi online zu haben. ((Herr E nickt)) Und unser Dozent hat eh immer so ein Inverted-Classroom-Ding gefahren. Das heißt, man musste es eh selber lesen und eh selber die Übungen machen. Und da war das jetzt auch von Mathe 1, das ja in Präsenz war, gar nicht so der Umstieg. Und das hat, muss ich echt sagen, sehr gut funktioniert. (FGS5: 34)

Eine Offenheit gegenüber Neuem, wie dem Einsatz von digitalen Formaten in der Präsenzlehre, wurde in einer Fokusgruppe ebenfalls gefordert, sodass in Zukunft eine Vielfalt der Lehrformate und eine Abwechslung innerhalb der Lehre entstehen. Vor allem wurden die digitalen Veranstaltungen, die außerhalb des regulären Studiums stattfanden, als besonders wertvoll und erhaltungswürdig empfunden. Hier wurde besonders hervorgehoben, dass durch die Vorträge, Workshops und Diskussionsrunden mit externen Beteiligten das Netzwerken erleichtert wurde, ein fachlicher Austausch stattfand und vor allem bisher nicht erreichbare Expert*innen als Referent ${ }^{*}$ innen eingeladen wurden: „Das bedeutet, wir haben dann ganz andere Möglichkeiten, an Fachwissen ranzukommen, ((Frau G nickt)) an Austausch, also einen fachlichen Austausch, der sozusagen uns das neueste Wissen mit reinbringt" (FGS3: 113). Zu den Angeboten, die außerhalb der regulären Lehre stattfanden, gehörten für die Studierenden auch Tipps und Tricks für erfolgreiches digitales Lernen oder das einfache, gesundheitlich korrekte und günstige Einrichten des Homestudying-Platzes, welche jedoch noch nicht an allen Hochschulen angeboten wurden.

Die Möglichkeit, Prüfungen online abzulegen, z.B. Klausuren, Hausarbeiten oder Präsentationen, waren ebenfalls in der Post-Corona-Zeit gewünscht. Jedoch wurde hier auch auf die ungleichen Voraussetzun- 
gen und Fähigkeiten hingewiesen. Ein ${ }^{\star} e$ Studierende ${ }^{\star} r$ beschrieb die Gedanken dazu wie folgt:

Und wenn das eben in dem Modus war, dass die Zeit knapp war ((Herr J nickt)) und Open Book, dann war es halt irgendwie insofern, fand ich, schwierig. Die haben halt dann einfach zusammengearbeitet, die haben sich noch jemand anderen der das vielleicht schon im letzten Semester geschrieben hat, dazugeholt und haben die Aufgaben gemeinsam bearbeitet und das benachteiligt halt tatsächlich diejenigen, die nicht so gut vernetzt sind oder vielleicht daheim sind oder nicht so viele Freunde da haben, die das Gleiche machen. Also insofern ist es dann schon, finde ich, deutlich ungerechter. (FGS2: 80)

Aus den Herausforderungen, Chancen und Wünschen hinsichtlich digitaler Lehre gingen die Handlungsempfehlungen seitens der Studierenden deutlich in eine Richtung. Ein deutliches Votum für ein Zurück zur Präsenzlehre wurde von allen befragten Studierenden mehrfach betont. Ein großer Vorteil der Präsenzlehre war das Haptische und Praktische im Studium, welches hauptsächlich durch gemeinschaftliches Arbeiten und Lernen, den direkten Umgang mit Materialien sowie das Experimentieren im Labor gekennzeichnet war. Dieses gemeinschaftliche Lernen, der Input und die Hilfestellungen von anderen Studierenden spendeten Motivation und machten eine HAW aus.

Also ich würde tatsächlich sagen, so dieser persönliche Bezug einfach, die Motivation, die man irgendwie dann auch kriegt, der Zuspruch, wenn etwas nicht klappt, irgendwie das, diese Vermittlung von dem, dass man das eben nicht alleine machen muss, dass man da die Rückmeldung hat, dass man die Leute dann auch irgendwie kennt. (FGS7: 166)

Die didaktische Weiterentwicklung wurde ebenfalls als Handlungsempfehlung in Richtung der Lehrenden von den befragten Studierenden genannt. So sollten die Vorteile der Flexibilität in Zeit und Ort für die Hochschullehre der Zukunft genutzt werden. Des Weiteren sollte das Digitale verwendet werden, um die Präsenzlehre zu verbessern.

Und ja, ansonsten würde ich schon auch sagen, dass ich allgemein finde, dass Schulen, Hochschulen, Universitäten in Deutschland ziemlich rückständig waren und eigentlich immer noch sind, was Digitalisierung und so was angeht. Und dass man das jetzt auf jeden Fall nutzen kann, um auch die Präsenzlehre zu verbessern. (FGS7: 284)

In den folgenden Semestern wünschten sich einige Studierende eine abwechslungsreiche Lehre mit synchronen Veranstaltungen. Es zeigte 
sich in der Analyse, dass sich laut den Studierenden die digitale Herangehensweise der Lehrenden deutlich verbesserte und vermehrt auch verschiedene digitale Werkzeuge verwendet wurden.

Aber stattdessen sollte man auch mehr auf die Kommunikation zwischen den Studierenden eingehen und dass das eben mehr gefördert wird, ((Herr C nickt)) und gleichzeitig auch den Profs irgendwie vermitteln, dass sie ihr Onlinekonzept nicht genauso machen wie ihr Präsenzkonzept, nur dass sie sich dabei aufnehmen. (FGS6: 128)

Für die Post-Corona-Hochschule, so einige der befragten Studierenden, sollte Praktisches wie Gruppen-, Labor- und Projektarbeiten wieder vor Ort stattfinden.

\section{Zusammenführung}

Zusammenführend zeigte sich, dass die Flexibilität der digitalen Lehre für die Teilnehmenden der Fokusgruppen einen Vorteil darstellte, der auch Chancen für die zukünftige Lehre beinhaltet. Dabei wurden einige Aspekte beidseitig genannt, andere wiederum um die Sicht der Lehrenden ergänzt (s. Abbildung 17).

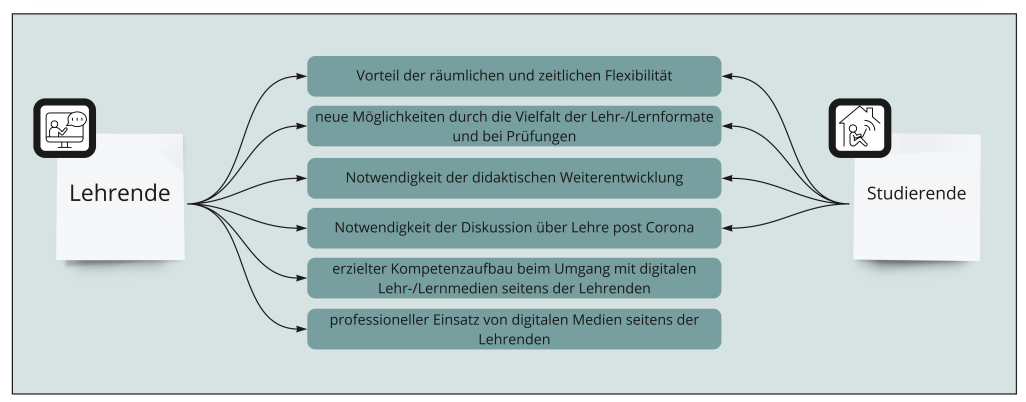

Abbildung 17: Blick in die Zukunft - didaktische und technische Aspekte

So ermöglichte die örtliche und zeitliche Flexibilität nicht nur, in der eigenen Geschwindigkeit zu lernen, sondern auch eine bessere Vereinbarkeit von Familie und Beruf bzw. Studium. Durch die digitale Lehre in den Corona-Semestern wurde die Notwendigkeit einer didaktischen Weiterentwicklung der zukünftigen Präsenzlehre sichtbar. Die Studie- 
renden erachteten zusätzlich die didaktische Weiterentwicklung der digitalen Lehre hinsichtlich des dritten Corona-Semesters als wichtig. In Bezug auf die Präsenzlehre waren sich Studierende und Lehrende einig, denn beide Gruppen wollen zurück an die Hochschule, um dort wieder vor Ort das Lehren bzw. das Studieren erleben zu können. Jedoch ist es kein Zurück in den Zustand vor Corona, sondern in ein Modell von hybrider Lehre, welches sich alle Teilnehmenden der Fokusgruppen wünschten. Vor allem die praktischen Fächer des Studiums, die in Gruppen- und Projektarbeiten oder Laborpraktika durchgeführt wurden, sollten in Zukunft wieder in Präsenz stattfinden, da hier ein interaktiver Austausch besser funktioniere und dadurch das Lernen unterstütze. Auch in diesem Punkt herrschte Einigkeit in allen Fokusgruppen.

\subsubsection{Kommunikation und Interaktion}

\section{a. Perspektive der Lehrenden}

Ich meine, ich habe so was von Bock, im Hörsaal zu stehen und da ((Herr $\mathrm{M}$ lacht)) rumzulaufen und rumzukaspern. Ich glaube, diesen Endorphinschub, den man da hat, der wird mindestens fünf Jahre andauern. ((alle lachen)) Und ich glaube, so wird es den meisten der Kollegen gehen. Ich meine, hier klang das ja auch schon an so bescheiden: Wir wollen mit Menschen zu tun haben. Ich meine, wir wollen doch im Hörsaal sein und da rumturnen und da unsere Show abziehen und den Studenten was beibringen. Das ist doch unsere Passion. (FGL2: 96)

Aus Sicht der Lehrenden lebte der Lehrberuf gerade von dem Kontakt zu den Studierenden im Hörsaal, deren direkten Reaktionen zu sehen und darauf reagieren zu können, wie ein ${ }^{\star} e$ Lehrende ${ }^{\star} r$ im obigen Zitat pointiert beschreibt.

Es wurde von den Lehrenden als zentraler Wunsch formuliert, dass Präsenzlehre wieder benötigt wird. Sie sahen das digitale Unterrichten nicht als einen zukünftigen Dauerzustand. „Wir sind Menschen, wir sind soziale Wesen“ (FGL3: 106). Lehren und Lernen geschieht im Austausch zwischen Lehrenden und Studierenden sowie zwischen den Studierenden durch die physische Begegnung. Im Digitalen war alles möglich, es wird aber eher als „Krücke“ (FGL3: 106) und nicht 
als wirkliche Alternative empfunden. Insbesondere für das vertiefte Lernen und Verstehen waren der direkte Austausch und die gemeinsame Lernatmosphäre notwendig. „Eine ganz starke Kommunikation von Gehirn zu Gehirn, also rein auf der rationalen Ebene. Und vieles, was eben zum Lernen auch dazugehört im Bereich von Emotionen etc., bleibt halt auf der Strecke" (FGL1: 43). Hierzu gehörte neben dem Austausch zwischen Lehrenden und Studierenden auch der Aspekt des Peer-to-Peer-Lernens zwischen den Studierenden, dass die Studierenden mit dem Sitznachbarn reden können, ob die anderen den Lehrinhalt ebenso nicht verstanden haben - dies sei digital nur bedingt umsetzbar.

In der Zusammenarbeit gibt es gute Ergänzungsmöglichkeiten durch die digitalen Kommunikationsformate. Dokumente könnten jetzt gemeinsam mit externen Partner*innen über Webanwendungen wie SharePoint bearbeitet werden, sodass auf das Verschicken von Dokumenten per E-Mail verzichtet werden kann. Ein Teil der Lehrenden hat mit digitalen Einzelbesprechungen sehr gute Erfahrungen gemacht, so waren in digitalen Sprechstunden auch der Kontakt und die persönliche Betreuung besser, da bei virtuellen Einzelgesprächen niemand unangemeldet ins Büro kam oder eine gestörte Gesprächsatmosphäre durch eine Zweierbüro-Situation vor Ort das Gespräch weniger offen gestaltete.

Mit Einzelnen da sehr intensiv zu diskutieren und die Nähe fast größer war, das erlebe ich tatsächlich auch so. Typischerweise habe ich, wenn ich es dann eingeplant habe, die Zeit am Rechner, auch mehr Zeit und mehr Muße als eben auf dem Flur. (FGL4: 29)

Aus Sicht der Lehrenden sollten digitale Sprechstunden, Besprechungen und Abschlussarbeitsbetreuung als Alternativmöglichkeit beibehalten werden. Ebenso würde ein Teil der Lehrenden gerne die Möglichkeit der digitalen, interaktiven Gruppenarbeit über Breakout Rooms und Whiteboards beibehalten.

Das ist eben dieses Thema Gruppenarbeit. Ich kann virtuell plötzlich einen Raum von 100 Leuten in zehn Gruppen aufteilen, kann denen einen Flipchart zur Verfügung stellen, Post-ist.ØLäuft super. Das könnte ich niemals im richtigen Vorlesungsbetrieb. Alleine die Räume aufzutreiben, wäre der Horror. ((Herr M lacht)) Und die ganzen Moderatorenkof- 
fer und so. Das geht deutlich besser, deutlich besser. Man kann sich da richtig neue Sachen ausdenken. (FGL2: 42)

Einigkeit bestand darüber, dass sich insbesondere die Erst-/Zweitsemester mit digitalen Vorlesungs- und Austauschformaten schwertun, da sie weder die Hochschule noch Lehrende und Studierende kannten und nicht einschätzen konnten, wie an der Hochschule gelernt wird. Hier war die Hemmschwelle größer, sich zu Wort zu melden, etwas beizutragen oder etwas zu fragen. Hier bedarf es zuerst eines persönlichen physisches Kennenlernens.

\section{b. Perspektive der Studierenden}

Ich würde halt sagen, dass gerade dieses Zwischenmenschliche, das wird auch online niemals so sein wie präsent. Also da können wir noch so lange irgendwelche Sachen planen und üben und sonst noch was, es wird nie so sein. Und das ist halt in uns Menschen, würde ich sagen, einfach so veranlagt, dass man eben eine Gruppe sein möchte. (FGS7: 237)

$\mathrm{Zu}$ einer HAW, die vor allem eine Präsenzhochschule ist, gehört das Miteinander, die Interaktion und der Austausch vor Ort. Viele befragte Studierende wünschten sich, dass die Rückkehr in die Präsenzhochschule wieder zu einem gemeinschaftlichen Hochschulalltag mit physischer Interaktion führt. In den Fokusgruppen wurde deutlich, dass die Kommunikation in Präsenz größere Vorteile als die im Digitalen habe. So funktionierte das gegenseitige Kennenlernen besser in physischer Präsenz. Zufallsbegegnungen gibt es nicht im Digitalen, weshalb ein gemeinsamer Hochschulalltag vor Ort ebenfalls wichtig für das Gemeinschaftsgefühl ist. Und das Zwischenmenschliche und Familiäre ist nur in der Präsenzhochschule spürbar. Dies drückte eine Teilnehmende wie folgt aus:

Ich würde mir aber auch wünschen, dass, ja, man das ((lacht)) Digitale irgendwie dann doch auf ein notwendiges Minimum, sage ich mal, reduziert, dass man auch hier wieder so ein bisschen dieses Interaktive, das Zwischenmenschliche, dieses Familiäre halt doch ganz schnell wieder wiederbeleben lässt. (FGS1: 161)

Für die Zweitsemester war das Thema Zwischenmenschlichkeit noch von größerer Bedeutung, obwohl sie es vor Ort noch nicht erlebt haben. 
Und das Zweite, glaube ich, ist einfach, dass dieses Zwischenmenschliche, ob jetzt zwischen den Dozenten oder zwischen den Dozenten und den Studenten oder zwischen den Studenten allgemein, dass eben diese Zwischenmenschlichkeit einfach genauso wichtig ist eigentlich wie der Inhalt ((Frau $\mathrm{R}$ nickt)) und dass da eben auch viel Wert daraufgelegt werden sollte. (FGS7: 235)

Ein weiteres Thema für die Zweitsemester war auch das Kommunizieren auf Augenhöhe mit den Lehrenden.

Ich würde mir auch wünschen, dass halt die Gemeinschaft zwischen den Studierenden untereinander gefördert wird, aber auch dass die Beziehung zwischen den Studierenden und den Dozenten verbessert wird und dass man so auf einer Ebene miteinander kommunizieren kann. (FGS7: 293)

Auch die anderen Teilnehmenden der Fokusgruppen, die bereits Erfahrung mit dem Studium vor Ort an der Hochschule hatten, reflektierten die Situation der Erst- und Zweitsemester, die die eigene Hochschule nicht kannten und auch die Mitstudierenden, wenn überhaupt, nur zu den Prüfungen sahen. Daraus ergab sich eine wichtige Handlungsempfehlung der Studierenden, die bereits Präsenzhochschule erlebt hatten, dass Erst- und Zweitsemester im Bachelor und im Master, in der Präsenzlehre bevorzugt werden, damit sie sich gegenseitig, die Lehrenden und die Hochschule kennenlernen konnten. Ein Studierender beschrieb dazu, dass seine Kontakte aus der Einführungswoche stammten: „Und das sind, ich muss auch wirklich sagen, der Großteil der Menschen bei uns, die da Kontakte aufbauen, die stammen alle aus der Ersti-Woche“ (FGS3: 86). Damit betonte er, wie wichtig dieses erste Zusammentreffen an der Hochschule auch für den Lernerfolg ist. Darüber hinaus wünschten sich einige Studierende, dass die Studienreisen und Aktivitäten, die aufgrund der Corona-Pandemie nicht stattfanden, in der Post-Corona-Hochschule nachgeholt werden.

Ja, also ich würde mir wünschen, dass zum Beispiel diese Studentenreisen ..., dass so was dann halt trotzdem nachgeholt wird, weil solche Reisen, ich denke, das verbindet da die Studenten halt noch mehr, als einfach, auch wenn es wieder präsent wäre, in einem Vorlesungssaal zusammenzusitzen. (FGS7: 284)

Der Austausch im Digitalen habe aber auch seine Vorteile, wie die schnellere, direkte und unmittelbare Kommunikation mit den Kommiliton*innen und auch teilweise mit den Lehrenden. „Und auch ... 
mit anderen Mitstudenten konnte man einfach wirklich kurz mal anrufen, fünf Minuten was reden und direkt mit dem nächsten wieder telefonieren“" (FGS6: 20).

Der regelmäßige Austausch, der vor Corona nicht gegeben war, war durch die digitalen Austauschmöglichkeiten wie Discord im CoronaSemester entstanden und unterstützte so das Lernen. Insgesamt sind sich die befragten Studierenden einig, dass das gegenseitige Kennenlernen und der physische Kontakt, Austausch und Interaktion miteinander in der Post-Corona-Präsenzhochschule von ausschlaggebender Bedeutung für ein Studium an einer HAW sind. Denn die Studierenden entschieden sich zu Beginn ihres Studiums für ein praxisorientiertes vor Ort stattfindendes Studium mit allem, was noch zum Studierendenleben dazugehöre, vor allem dem Gemeinschaftsgefühl. Wie bereits im Kapitel 5.3 zur Gesamtsituation deutlich wurde, fehlte den Studierenden das Studierendenleben enorm. Dadurch sank sogar die Motivation für das Studium, denn gerade Events wie das Feiern der abgeschlossenen Prüfungsphase fehlten, sind aber mit für die Motivation am Studium verantwortlich.

\section{c. Zusammenführung}

Zusammenfassend konnte für das Thema Kommunikation und Interaktion festgestellt werden, dass die Fokusgruppen einen Verlust der bisher als selbstverständlich erachteten Austauschmöglichkeiten spürten. Dies ließ sich an folgenden, wiederkehrenden Aspekten erkennen (s. Abbildung 18).

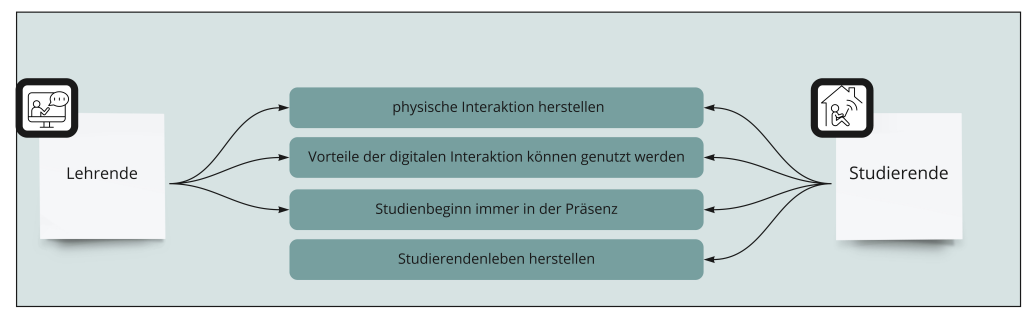

Abbildung 18: Blick in die Zukunft - Aspekte der Kommunikation und der Interaktion 
Die physische Interaktion und Kommunikation waren ausschlaggebend für eine gelingende Lehre, so die Lehrenden. Die digitale Kommunikation brachte in bestimmten Situationen neue Möglichkeiten wie digitale Beratungen, Gruppenarbeiten und die eigene Erreichbarkeit. Jedoch fehlte insgesamt der physische und interaktive Austausch. Dieser Austausch war für die Studierenden gerade in den Gruppenund Projektarbeiten von großer Bedeutung für den Lernerfolg, weshalb das Öffnen der Hochschule für kleine Gruppen noch während der Pandemie gewünscht wurde. Für Studierende kam noch hinzu, dass sie mit der Präsenzhochschule auch das Leben rund um das Studium verbinden. Hier fehlte vor allem das Gemeinschaftsgefühl, welches durch den interaktiven Austausch an der Hochschule entstand und deshalb in Zukunft unbedingt wieder unterstützt und gefördert werden muss. Insgesamt waren sich jedoch beide Akteur*innengruppen darin einig, dass die digitalen Austauschmöglichkeiten post Corona unbedingt beibehalten und in den Lehr-/Studienbetrieb integriert werden sollten, denn sie unterstützen die Lehre in den digitalen Corona-Semestern und könnten dies auch im zukünftigen Lehrmodell tun.

\subsubsection{Rahmenbedingungen/Strukturen}

Wie es in den Post-Corona-Semestern weitergehen sollte, beschäftigte alle Teilnehmenden der Fokusgruppen. Im Folgenden werden die Handlungsempfehlungen wiedergegeben, die die Studierenden und Lehrenden bezüglich der Struktur bzw. Rahmenbedingungen für die zukünftige Hochschullehre geben.

\section{a. Perspektive der Lehrenden}

Wir haben uns ad hoc bewegt in einem Ausmaß, was unvorstellbar war für mich vorher. Und da muss ich auch wirklich sagen, was die Hochschule, die Verwaltung, die Kollegen, aber auch die Lehrbeauftragten auf die Füße gestellt haben in dieser Zeit, das ist für mich schon phantastisch. Und ich erhoffe mir für die Zukunft, dass uns ein Stück weit diese Dynamik bleibt und auch etwas von dem ((lacht)) Aufbruchsgeist. Also ansonsten, als Hochschule habe ich die Institution immer relativ formal und undynamisch erlebt. (FGL W: 100) 
Das Zitat verdeutlichte, dass die entstandene strukturelle Weiterentwicklung der Hochschule von den Lehrenden als Innovationschub und als Aufbruchsstimmung gesehen wurde.

Darin sahen die Lehrenden eine große Chance und waren sich weitgehend einig, nicht wieder in die „alte Welt“ (FGL7: 110) zurückkehren zu wollen. „Also dass man jetzt einfach diese Flexibilität auch im Kopf hat und die hoffentlich behält ((Herr P nickt)), ((lacht)), das würde ich mir wünschen" (FGL8: 103). Positiv empfanden sie auch, dass Entscheidungen, z.B. bei Beschaffungen, unbürokratischer und schneller in den Corona-Semestern abliefen, was sie sich auch zukünftig wünschten. Diese Schnelligkeit sollte beibehalten werden. „Und manchmal würde ich mir wünschen, dass wir weniger Regeln haben, sondern einfach mehr ausprobieren können und weniger Formalismen tätigen müssen" (FGL6: 83). Vermieden werden sollte dabei eine Überregulierung, die dann wieder als Innovationsbremse wirke, z.B. hinsichtlich der Deputatsabrechnungen. Hier fehle ein aktualisiertes Vorgehen für die digitale Lehre.

Irgendwas, was tatsächlich auch als Innovationsbremse wirkt, weil man die Leute quasi für alles, was vom Status quo abweicht, natürlich erst mal in die Bringschuld stellt, zu rechtfertigen, warum sie sich denn damit keinen persönlichen Vorteil verschaffen, weil das ja das ist, was wir alle primär tun wollen. (FGL6: 83)

Damit verbunden formulierten die Lehrenden auch den Wunsch nach Anerkennung und Würdigung des Mehraufwands. „Okay, habt ihr toll gemacht, ja? Wir stemmen wirklich eine komplette Lehre aus Null“ (FGL3: 128). Dagegen würde über die Formalisierung der Deputatsabrechnungen stattdessen Misstrauen gesät. Jedoch wurde dafür geworben, dass „da ... ein Grundvertrauen da sein“ (FGL4: 80) sollte. Weiterhin waren noch lange nicht alle Lerninhalte in digitale Formate überführt worden, sondern dies geschah zusätzlich $\mathrm{zu}$ eigentlichen Semesterwochenstunden.

Ein Modell-Mix wurde von allen Lehrenden befürwortet, im Sinne eines hybriden Modells der Hochschullehre, in welchem sowohl Präsenz- als auch digitale Elemente vorhanden sein sollten. Digitale Lehre sollte als ein selbstverständlicher Teil in das Gesamtkonzept der Hochschule integriert werden. „Die Vorteile dessen, was wir jetzt im digitalen Raum erlebt haben, einfach mit rübernehmen und das 
integrativ behandeln, das Thema" (FGL1: 94). Wie dieses Modell dann tatsächlich aussehen sollte, dazu gingen die Meinungen der Lehrenden auseinander und sie entwickelten unterschiedliche Ideen. Die Lehrenden mochten selbst entscheiden, welches Format sie wann wie nutzen. Einig waren sie sich, dass ein vertieftes Verstehen in Präsenz stattfinden und dass die Auswahl der Formate unter didaktischen Gesichtspunkten erfolgen muss. So kamen Vorschläge, dass es feste Tage für digitale bzw. Präsenzlehre geben könnte oder Grundlagenvorlesungen digital zu gestalten wären und alle praktischen Formate wie Projekte oder Labore vor Ort in Präsenz stattfinden sollten.

Okay, ihr bleibt einen Tag lang komplett zu Hause in der Vorlesung, in der Übungsgruppe, aber dann, wenn ihr an die Hochschule kommt, dann findet die Präsenz auch so statt, dass die Präsenz Sinn macht. Dann sitzt ihr nicht 130 Leute in einem Hörsaal, ja? ((Frau T nickt, zeigt Daumen hoch)) Schlaft ein und guckt mir zu, wie ich einen Fehler an die Tafel rechne, ja ((Frau T lacht)). (FGL3: 102)

Die Lehrenden wiesen auch auf den praktischen Aspekt der Raumsituation hin, welche sich durch die Nutzung von digitalen Formaten entspannen würde, denn in einigen Hochschulen waren zu wenig große Vorlesungsräume vorhanden. Eine Herausforderung sahen die Lehrenden bei möglichen Hybridmodellen in der stundenplanmäßigen Organisation der unterschiedlichen Formate: „Und dann eben auch stundenplanmäßig so organisieren, dass es für alle Beteiligten eine verlässliche Konzeption ist" (FGL3: 123). Dies bedarf dann entsprechender formaler Anpassungen in den Studien-/Prüfungsordnungen oder Studiengangssatzungen.

Ich wünsche mir halt dann auch eine vernünftige Aufteilung zwischen digitaler und Präsenzlehre und dass man dann auch ein bisschen so in der SPO niederschreibt, ja, dass man das, dass man diese Flexibilität dann halt hat, dass man zwischen beidem ((lacht)) wählen kann sozusagen. (FGL7: 113)

Hier war aus Sicht der Lehrenden noch Regelungsbedarf bezüglich einheitlicher Rahmenbedingungen bei der Nutzung der digitalen Werkzeuge und Datenschutzregelungen „ohne immer gleich damit rechnen zu müssen, dass uns der Datenschutzbeauftragte oder irgendwelche anderen Leute auf die Füße steigen“" (FGL6: 87). Damit verbunden muss es auch Regelungen für Onlineprüfungen oder Abrech- 
nungen geben. Andererseits diskutierten die Lehrenden auch, dass zu viele zentrale Regelungen wiederum die Kreativität schmälern. Es sei das Spannungsfeld zwischen Aufrechterhaltung der Vielfalt und einer einheitlichen Vorgehensweise.

Auch Bedenken Richtung Hochschulfinanzierung wurden geäußert, vor allem bezüglich der Möglichkeit, Räume oder Personal durch digitale Lehre einzusparen. „Ich glaube, da muss man auch sehr achtsam sein, aber das/ achtsam, aufmerksam. Aber das dürfte einen ja nicht darin behindern, die Werkzeuge so zu nutzen, wie sie richtig sind" (FGL4: 84). Bei der Weiterentwicklung wäre es hilfreich, auch über den Tellerrand hinauszuschauen und von anderen Ländern und Beispielen zu lernen, die bereits seit vielen Jahren Erfahrungen mit Fernlernen oder Fernstudium machten.

Hybride Formate könnten auch helfen, allgemein besser auf die Diversität der Studierenden einzugehen, z.B. wenn Studierende nebenher in Vollzeit arbeiten, Kinder erziehen oder gesundheitliche Beeinträchtigungen haben.

Das ist das, was ich so gerne mit rüber nehmen würde, sozusagen, dann in die hybride Lehre, dass wir hier wesentlich flexibler und viel, ja, mehr auf die einzelnen Studenten und deren Lebenssituation tatsächlich reagieren können. Also seien es jetzt eben Studierende, die viel arbeiten müssen, weil es eben die in Anführungszeichen klassischen Studierenden so ja nicht gibt, sondern die unterscheiden sich ja wirklich in einer großen Zahl. Dass es da eben welche gibt, die nur arbeiten, sondern eben auch Kinder erziehen müssen und im Beruf, im Vollberuf stehen, ja? Also all das, glaube ich, ist so was, was ich gerne mitnehme und was ich sehe, was durch digitale Medien möglich ist, hier wirklich auf die Diversität der Studierenden eingehen zu können. (FGL2: 84)

Andere Lehrende wiesen darauf hin, dass in diesem Zusammenhang auch die international Studierenden im Blick behalten werden sollten, die während der Corona-Semester nicht an den HAWs studieren konnten. Für manche Hochschulen stellten die international Studierenden auch eine wichtige Zielgruppe für bestimmte Studiengänge dar und nun fehlte diese Gruppe.

Hinsichtlich der strukturellen Weiterentwicklung der Studiengänge bieten aus Sicht einiger Lehrender die digitalen Lehrformate neue Möglichkeiten bei der Umsetzung von Teilzeitstudienmodellen neben 
den bestehenden Vollzeitstudienmodellen, die bisher aufgrund von mangelndem Personal und Ausstattung nicht durchgeführt werden konnten. „Diese Digitalisierungsgeschichte wird neue Möglichkeiten in diese Richtung eröffnen. $\mathrm{Ob}$ und wie das kommt, weiß ich nicht, aber da sehe ich mindestens eine gewisse Chance" (FGL2: 80).

Die Digitalisierung von Verwaltungsprozessen, wie beispielsweise die Möglichkeit, digital unterschreiben zu können, Dokumentationen oder Abrechnungen per E-Mail statt über Hauspost erledigen zu können, wurde als eine Erleichterung bei der Lehrtätigkeit empfunden und auch für die Zukunft gewünscht. „Und erfreulicherweise ist es bei uns auch tatsächlich so eingerichtet, dass man die ganzen Formalkramgeschichten tatsächlich dann auch online bzw. per Mail machen kann" (FGL6: 20).

Die Lehrenden diskutierten auch, inwiefern digitale Formate als ein mögliches Entscheidungskriterium der Studierenden bei der zukünftigen Auswahl ihrer Hochschule eine Rolle spielen könnten Die Fokusgruppen der Lehrbeauftragten brachten Sichtweisen von außen ergänzend hinzu. Sie diskutierten die Hochschulentwicklung post Corona auch insbesondere unter dem Thema Wettbewerbsfähigkeit der HAWs gegenüber anderen Hochschulformen. Hier war es wichtig eine gute technische Ausstattung und einheitliche Standards an den Hochschulen zu haben. Die Lehrbeauftragten waren sich einig, die Lehrtätigkeit nicht wegen der Bezahlung zu machen, sondern aus Spaß am Umgang und Austausch mit jungen Menschen. Eine Lehrbeauftragte wies auch darauf hin, dass die Bezahlung an den HAWs im Vergleich zu den privaten Hochschulen niedriger sei, zusätzlich böten die privaten Hochschulen noch mehr Unterstützung an. Wichtig war aus Sicht dieser Lehrbeauftragten, die Studierenden auf die digitale Arbeitswelt entsprechend vorzubereiten, da in vielen Arbeitsbereichen die Digitalisierung längst viel weiter fortgeschritten sei als an den Hochschulen. „Also wenn ich das jetzt nur so aus der Vogelperspektive sage, dann muss ich zu mir sagen: Es wurde Zeit und es war lange überfällig, dass auch die Hochschulen sich dem Online-Thema annehmen“ (FGL7: 82).

Die Lehrbeauftragten beider Fokusgruppen favorisierten, wie die Teilnehmenden der anderen Lehrenden-Fokusgruppen, ein Mischformat 
oder wollten zurück zur Präsenzlehre. Wäre ein Teil der Lehre digital, würde es den Lehrbeauftragten mehr Flexibilität in der Vereinbarkeit mit ihrer anderen Berufstätigkeit bringen. „Dass ich nicht mehr 30.000 Kilometer Auto fahre, sondern nur noch 3.000, ist es nicht auch ein Geschenk für die eigene persönliche Lebenszeit“ (FGL7: 123). Wichtig ist in Zukunft das Kennenlernen der Studierenden in Präsenz und darauf aufbauend könnten auch digitale Elemente folgen.

\section{b. Perspektive der Studierenden}

Und ich denke, das ist für ganz viele Studierende wichtig, ob sie jetzt das Zimmer kündigen oder nicht, ziehe ich jetzt wieder zu den Eltern oder nicht. Und ich finde es jetzt wichtig, dass man einfach jetzt ein bisschen trotz diesen ungewissen Zeiten einfach ein Stück weit planen kann, wo man sich denn befindet. (FGS2: 157)

Für das unmittelbar nächste Semester (SoSe 2021) und auch das darauffolgende wünschten sich die Studierenden Planungssicherheit, die sie auch nachdrücklich betonten. Denn für Studierende, die am Hochschulstandort wohnen, stand von Beginn der Corona-Pandemie an die Frage im Raum, ob sie ihr Wohnen nicht wieder in das Elternhaus verlagern sollten. Mit der geforderten Planungssicherheit wurde auch ein Gefühl von Alltag in das Leben der Studierenden treten, was das Lernen erleichtern würde.

Also klar, die können nicht frei entscheiden, ob sie hybrid machen wollen oder dann in Präsenz was vorlesen wollen, aber diese Vorgabe, dass man sich einfach daran anpassen kann, das würde halt extrem helfen, damit man einfach ein bisschen selber sein Leben planen kann und sich darauf vorbereiten kann. (FGS2: 156)

Es wird als Anliegen geäußert, dass es auch unter Corona-Bedingungen möglich sein sollte, in kleinen Gruppen an der Hochschule zu lernen. Auch die Werkstätten und Labore sollten im unmittelbar nächsten Semester bereits für die Studierenden nutzbar sein. Dies würde ebenfalls strukturelle Rahmenbedingungen benötigen, denn die Hygienekonzepte für die Präsenzprüfungen und Labore müssten verbessert werden: „Unser Hygienekonzept für die Prüfungen fand ich so, dass man es hätte auf jeden Fall besser machen können“ (FGS2: 162). 
Studierende wünschten sich darüber hinaus eine betont sinnvolle Aufteilung der Lehre in Präsenz und Digital: „Ich denke, ... dass man einfach durch die Fülle an Möglichkeiten, die es ja heutzutage gibt, da vielleicht einfach ein bisschen mehr mitnimmt und da in gewissen Bereichen halt auch mehr eingeht, sofern halt sinnvoll“ (FGS5: 132). Daraus ergibt sich ein Hybrid-Konzept, das ausgestaltet oder - wenn vorhanden - verbessert werden sollte. Ein ${ }^{\star}$ e Studierende ${ }^{\star} r$ äußert auch noch seine/ihre Bedenken bezüglich des zukünftigen Lehrkonzeptes: „Es gibt viele Möglichkeiten, es wäre dumm, aber leider zu erwarten, dass man sehr/ erst mal den Schritt zurück macht zur ((macht Geste für Anführungszeichen)) Normalität und dann wieder von null anfängt, sein eigenes Süppchen zu kochen“ (FGS3: 112).

Das zukünftige Konzept für die Lehre an HAWs sollte auch ein einheitliches Konzept sein. Ein ${ }^{\star} e$ andere ${ }^{\star} r$ Studierende ${ }^{\star} r$ machte ebenfalls auf die Einheitlichkeit des zukünftigen Lehrkonzeptes und auf den Wunsch zurück in die Präsenzhochschule zu gehen, aufmerksam.

Also ich würde mir wünschen, dass wir zur normalen Präsenz, zum normalen Präsenzalltag wieder zurückkehren, dass wir einen belebenden Austausch wiederbekommen zwischen den Studierenden und den Dozenten, und ja, dass vielleicht das Digitale unterstützend mit übernommen wird, aber nicht der maßgebende Faktor wird, und dass es auch, ja, von der Hochschule und von allen Dozenten sozusagen eine einheitliche Marschrichtung gibt und nicht dann quasi einer macht nur Onlinevorlesung, einer macht nur Präsenzvorlesungen. Genau, dass es da ein einheitliches dann Konzept wieder gibt und, ja, die Onlinelehre vielleicht ergänzend mitwirken kann. (FGS1: 165)

Insgesamt wünschten sich die Studierenden, dass die eigenen Hochschulen die aktuelle Situation nutzen, um die Hochschule als Institution $\mathrm{zu}$ revolutionieren, und dass eine bessere Kooperation zwischen den Hochschulen, aber auch zwischen den Gremien stattfindet.

$\mathrm{Na}$, wir erfinden jetzt das Rad neu und machen das für uns und na ja, wir haben ja schon immer so unser eigenes Süppchen gekocht und jeder, jeder hat Angst, dass die Konkurrenz einen aushorcht ((Herr $\mathrm{H}$ lacht)) oder irgendwelche Informationen ((Herr I lacht und nickt)) finden könnte. Und ich fand das so schädlich, weil, wir haben auf Studierendenebene gemerkt, wie wichtig Kooperation ist. (FGS3: 113)

Zwei Handlungsempfehlungen richteten sich auch an die politischen Entscheidungsträger. Die eine, sehr praktische Empfehlung fordert den 
Ausbau der Internetleitungen in Deutschland, während die andere Empfehlung eine deutliche Anerkennung, auch auf finanzieller Basis, der Leistungen der Lehrenden in der digitalen Lehre fordert.

Dass das Hochschulgesetz zwar angepasst wurde, auch zum Thema Prüfungsformen. Also ich kriege es ja mit, die finanzielle Diskussion: Wie rechtfertige ich und wie verbuche ich asynchrone Lehre? Also ein Video zu schneiden, ist einfach mindestens das Doppelte, eher das Drei- bis Fünffache an Aufwand von einer Vorlesung, die ich vor Ort halte. Heißt, ein Professor darf das quasi in diesem Semester nicht abbuchen, wenn er für eine Vorlesung das Fünffache an Aufwand hat, und also Professor für alle Lehrmenschen, aber die nächsten fünf Semester oder zehn Semester hat er quasi keine Anpassungen mehr, je nach Fach, oder nur noch geringfügige, kriegt dann aber eins auf die Füße, weil er quasi die Vorlesung nicht hält, sondern nur Videos zeigt und Fragestunden macht. (FGS3: 97)

In diesem Zitat wird deutlich, dass sich Studierende Gedanken darüber machten, wie Lehrende die Anerkennung erhalten, die sie aus Sicht der Studierenden für die letzten und auch zukünftige Coronaoder hybride Semester verdienen. Wie wichtig im zukünftigen Lehrmodell die Möglichkeit des physischen Austauschs ist, zeigte sich in der Frage danach, ob der Anteil des Digitalen die Entscheidung für oder gegen eine Hochschule beeinflussen würde. Wenn sich die befragten Studierenden post Corona an einer Hochschule bewerben würden, dann würden sie sich für die Präsenzhochschule entscheiden, die mit unterschiedlich großen Anteilen des Digitalen die Lehre stemmen würde. Es sollte, so die meisten befragten Studierenden, auf jeden Fall oder eher eine Präsenzhochschule sein: „Und gerade ich will ja kein Fernstudium machen, deshalb will ich ja den Präsenzunterricht" (FGS7: 266). Einige Studierende merkten an, dass auf jeden Fall ein digitaler Anteil in der Lehre vorhanden sein müsste. Andere merkten an, dass der Studiengang, die Größe des Studiengangs oder das Lehrkonzept ausschlaggebend dafür sind, sich an der einen oder der anderen Hochschule zu bewerben. „Aber wenn das ein weiteres Kriterium wäre, zwischen zwei Unis, die exakt dasselbe/ Studiengang anbieten, dann würde ich auf jeden Fall die mit mehr Präsenz vorziehen“" (FGS5: 134). 


\section{c. Zusammenführung}

Für Lehrende wie Studierende sind HAWs Präsenzhochschulen und das müsste auch in Zukunft wieder so sein (s. Abbildung 19).

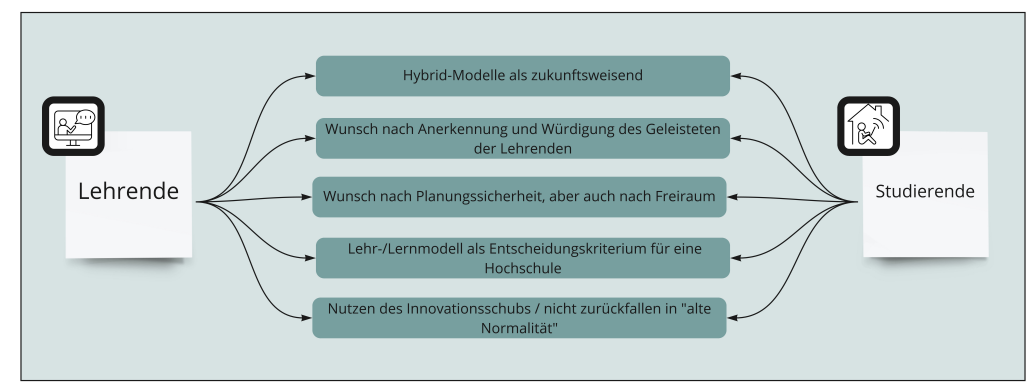

Abbildung 19: Blick in die Zukunft - Aspekte der Rahmenbedingungen und der Strukturen

Die Fokusgruppen stimmten dahingehend überein, dass das Digitale in der Hochschullehre nicht mehr wegzudenken ist. Sie sahen eine große Chance darin, die zukünftige Hochschullehre neu zu gestalten. So wurden unterschiedliche Ideen für ein Hybrid-Konzept vonseiten der Lehrenden geäußert und vonseiten der Studierenden Bedürfnisse bezüglich der zukünftigen Lehre. Das Hybrid-Konzept könnte sich, so die Lehrenden, in Tage der Präsenz- und Tage der digitalen Lehre oder auch in Theorievorlesungen und praxisorientierte Kleingruppenarbeit vor Ort teilen. Von den Studierenden wurde das Bedürfnis geäußert, gerade diese Kleingruppenarbeiten zu erleben. Für beide Gruppen, also Lehrende und Studierende, waren der physische Austausch und die physische Interaktion ein wichtiger Punkt, weshalb sich die Teilnehmenden ein Zurück an die Hochschule wünschten. Gerade für die Studierenden war ein einheitliches Konzept für die zukünftige Lehre ein Anliegen. Die Wettbewerbsfähigkeit der Hochschulen war für Lehrende wie Studierende ebenfalls ein wichtiges Thema, denn es wurde deutlich darauf hingewiesen, dass das zukünftige Lehrmodell ausschlaggebend für die Entscheidung für oder gegen eine Hochschule sein könne. 


\subsection{Fazit}

Mit Blick auf die Gestaltung des Kontingenzraums hat sich gezeigt, dass Studierende und Lehrende Wege gefunden haben, den neuen Möglichkeitsraum zu nutzen und zu gestalten (s. Abbildung 20).

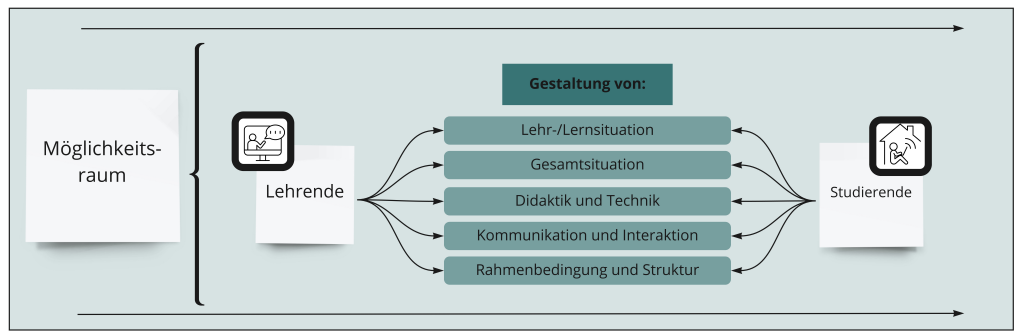

Abbildung 20: Gestaltungsfelder innerhalb des Möglichkeitsraums

In den Fokusgruppen zeigte sich, dass die abrupte Veränderung eines bisher für selbstverständlich erlebten hochschulischen Lehr-/Lernraums sowohl die Lehrenden als auch die Studierenden dazu brachte neue Handlungsstrategien sowohl hinsichtlich des Lehrens bzw. Lernens als auch für den sich ad hoc verändernden Lebensraum zu entwickeln. Die Lehrenden wurden selbst zu digital Lernenden, die Studierenden mussten insbesondere lernen, sich in ihren Studienalltag zu Hause einzufinden. Beide Akteur*innengruppen wurden ohne Vorbereitung ins Hometeaching bzw. Homestudying „hineingeworfen“. Häufig genannte Themen der Fokusgruppen mit Studierenden und Lehrenden waren der fehlende physische und interaktive Austausch sowie das als neu und ungewohnt erlebte digitale Setting. Wie wichtig die Kommunikation zwischen Lehrenden und Studierenden für das Lernen und Lehren an HAWs ist, wurde in den ersten zwei Corona-Semestern durch den plötzlichen Verlust der bisherigen Präsenzhochschule von allen persönlich erfahren. Einige konnten durch das Digitale des Studiums und der digitalen Lehre ihr reduziertes Leben in der Corona-Pandemie zwar besser organisieren, jedoch fehlte der Ausgleich, sodass körperliche und psychische Probleme spürbar in Form einer so geäußerten digitalen Erschöpfung auftraten. Für die unmit- 
telbare und Post-Corona-Zukunft wünschten sich die Teilnehmenden der Fokusgruppen ein hybrides Lehrkonzept, das die funktionierenden Elemente des Digitalen und der Präsenz in sich sinnvoll vereint. Eines haben alle Teilnehmenden der Fokusgruppen deutlich gemacht: Das praktische Lernen müsste vor Ort an der Hochschule gemeinsam mit anderen Studierenden stattfinden, ohne dass sie auf die neu gewonnene örtliche und zeitliche Flexibilität komplett verzichten möchten. Wie sich gelingende Lehre im Digitalen gestalten lässt, wird im nächsten Kapitel anhand von Fallbeispielen beschrieben. 


\section{Zugang 3: Good Practices und Potenziale digitaler Lehre}

Nach einer Annäherung an die Art und Bandbreite des Kontingenzraums und an Gestaltungsoptionen dieses Möglichkeitsraums durch Lehrende und Studierende in den Corona-Semestern soll in diesem Kapitel der Fokus auf eine weitere spezielle Zielgruppe gesetzt werden: diejenigen Lehrenden, die teils schon seit Jahren versuchen, die Digitalisierung in ihren eigenen Veranstaltungen und teilweise auch hochschulweit voranzubringen. So hatten diese Lehrenden im Zusammenhang mit der Ingangsetzung eines Innovationsprozesses an ihren Hochschulen eine treibende Kraft, indem sie - nicht erst pandemiebedingt - die Funktion eines "Early Adopters“ - im Sinne von (Rogers, 1995) einnahmen. Dieser aus der Diffusionstheorie stammende Begriff bezeichnet vor einem soziologischen, kommunikationswissenschaftlichen und betriebswirtschaftlichen Hintergrund Menschen, die in der Nutzung von jeweiligen technischen Neuerungen deutlich früher als die breite Masse dabei sind. In Rogers' Diffusionsansatz werden unter Innovationen Ideen, Prozesse oder Dinge verstanden, die für eine Gesellschaft oder eine soziale Gruppe subjektiv als neu wahrgenommen werden. Bestimmend für das Potenzial und die Verbreitung innovativer Prozesse sind dabei die Innovationseigenschaften, die Rahmenbedingungen im Prozess der Adoption sowie der individuelle Anspruch des Adopters, der diese Prozesse in verschiedenen Phasen durchläuft. An diesen Gedanken schließt sich der zentrale Aspekt der Einstellung gegenüber digitaler Lehre, der in Anlehnung an (Bolten \& Rott, 2018) bereits in der eingangs dargestellten Ausgangslage (s. Kapitel 1) hervorgehoben wurde: Eine positive persönliche Einstellung der Lehrenden gegenüber digitalen Medien ist maßgeblich entscheidend für die Umsetzung digitaler Lehr-/Lernsettings und besonders förderlich für eine positive Haltung hinsichtlich digitaler Hochschullehre, die so wie auch die Fallbeschreibungen hier in diesem Kapitel zeigen nicht zuletzt mit Innovationskraft einhergeht und zur Entwicklung von Good Practices führt. 
In den Corona-Semestern wurde von den hier befragten Lehrenden an Hochschulen in Baden-Württemberg teilweise auf sogenannte Leuchtturm- und Best-Practice-Projekte mit Fördergeldern aufgebaut, teilweise geschah die Verzahnung von digitaler Lehre und Präsenz in Eigeninitiative dieser Treiber. Diese Lehrenden hatten weniger Probleme, im Frühjahr 2020 ad hoc in die digitale Welt zu springen, da Vorerfahrungen und eine Offenheit gegenüber den neuen Anforderungen gegeben waren. Dies zeigte sich auch daran, dass sie nicht nur im Sommersemester 2020 Good Practices für die Lehre einbringen konnten, sondern auch ihre Veranstaltungen aufgrund der Erfahrungen weiterhin in den nächsten Semestern anpassten und optimierten.

Nach der Beschreibung des Untersuchungsgegenstands und der Methode in Kapitel 6.1 wird in Kapitel 6.2 die Frage nach bewährten Praxisansätzen in der digitalen Hochschullehre anhand dreier Fallbeschreibungen aufgegriffen: Mit Fokus auf bereits erprobten digitalen Lehr-/Lernformaten an acht HAWs in Baden-Württemberg wurden dazu hochschulübergreifend und anonym die Erfahrungen, Meinungen, Überzeugungen und Einschätzungen der befragten Lehrenden möglichst reichhaltig und tiefgründig rekonstruiert. Die Fallbeispiele mit den daraus abgeleiteten Good Practices bilden vier Schwerpunkte ab: Inverted Classroom mit Videos, Projektarbeit, Interaktion in der Onlineveranstaltung und digitale Prüfungsformate. In Kapitel 6.3 wird abschließend aus dem Blickwinkel der treibenden Lehrenden mit den Chancen und Wünschen für die zukünftige digitale Hochschullehre die Sichtweise auf die konkrete Lehrsituation und Didaktik abgerundet.

\subsection{Untersuchungsgegenstand und methodische Vorgehensweise}

Im Forschungszugang 3 lag das Hauptaugenmerk auf den Good Practices, sprich den in der digitalen Hochschullehre erprobten Praxisansätzen, die sich aus der Sicht der treibenden Lehrenden bewährt haben. Von forschungsleitendem Interesse waren dabei die folgenden Fragen (s. Abbildung 21). 


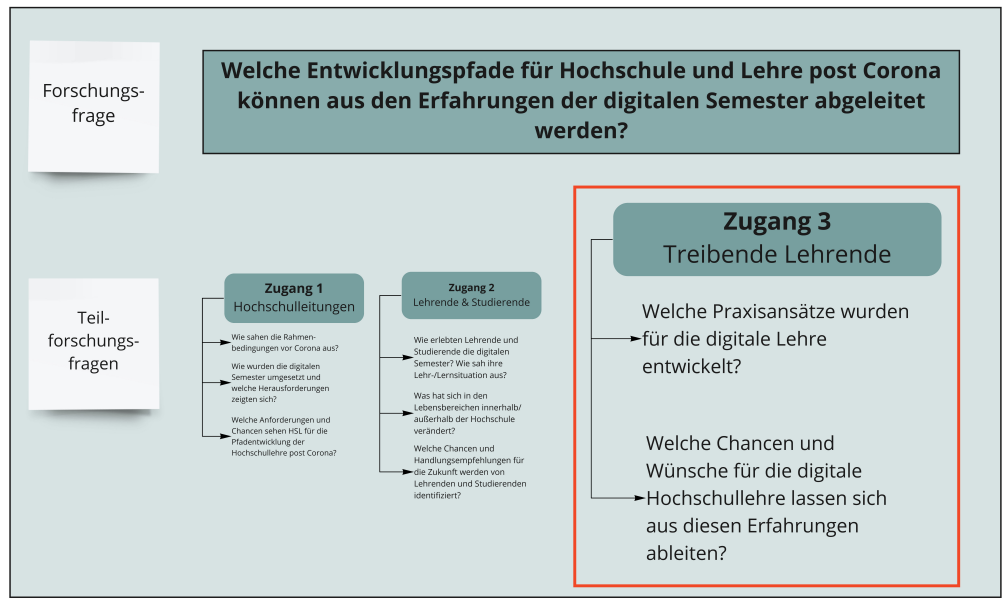

\section{Abbildung 21: Teilforschungsfragen Zugang 3 im Gesamtzusammenhang}

Für die qualitativ angelegte Datenerhebung wurden im Zeitraum von Dezember 2020 bis Januar 2021 acht Experteninterviews (Mayer, 2013) durchgeführt, im konkreten Fall leitfadengestützte Einzelinterviews mit ausgewählten Lehrenden, die aufgrund ihrer Vorerfahrungen mit digitaler Hochschullehre für den Untersuchungsgegenstand in diesem Zugang als besonders kompetent betrachtet wurden. Die Interviews wurden elektronisch aufgezeichnet, transkribiert und für die computergestützte inhaltsanalytische Auswertung anhand eines Kategoriensystems in Anlehnung an Kuckartz (s. Kapitel 3) aufbereitet.

Die Interviews (Durchschnittsdauer: 80 Minuten) waren themenfokussiert und bauten auf einem vorab entwickelten Leitfaden auf (s. Anhang 4), der sich aus den thematischen Schwerpunkten Mediendidaktik, Technisches \& Medienkompetenz und Kommunikation \& Interaktion zusammensetzte. Innerhalb dieser Themenbereiche bezogen sich die Leitfragen jeweils auf vier verschiedene Zeitphasen (s. Abbildung 22): 


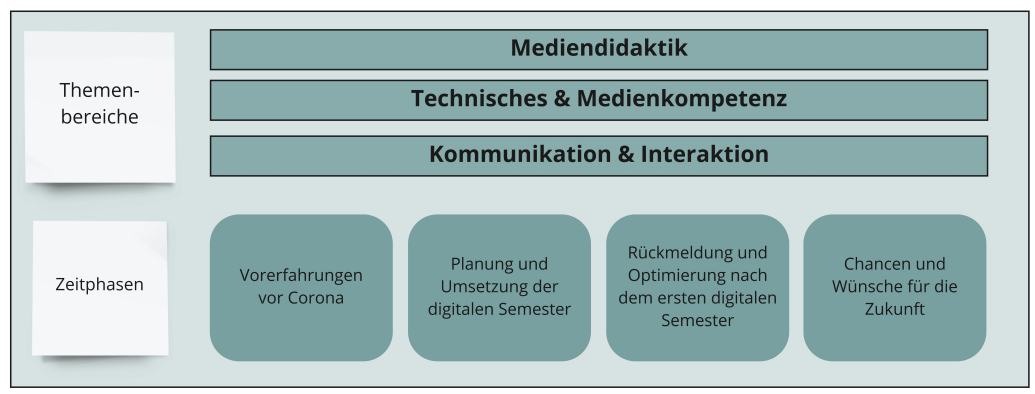

Abbildung 22: Leitfragenstruktur Zugang 3

Auf der Ebene der Mediendidaktik wurden die digitale Lehr-/Lernformate betreffenden Erfahrungen vor Corona erfasst und Aspekte der Planung und Umsetzung der Lehre mit digitalen Anteilen während der beiden Corona-Semester fokussiert. Im Bereich Technisches \& Medienkompetenz richtete sich die Aufmerksamkeit auf den Einsatz digitaler Werkzeuge in der Lehre sowie deren Funktionalität. Auf dieser Ebene wurde auch rückblickend auf unterstützende Supportangebote der Hochschulen zur Förderung medialer Kompetenz während der pandemiebedingten Umstellung auf die digitalen Semester eingegangen. Mit den Fragen zur Kommunikation \& Interaktion rückten die Möglichkeiten und Erfahrungen mit formellen und informellen Kommunikationswegen und Formen der Zusammenarbeit zwischen Lehrenden und Studierenden oder Studierenden untereinander in den Mittelpunkt. Parallel zu den hier skizzierten Bereichen wurden themenfokussierte Fragen zu Chancen und Potenzialen digitaler Hochschullehre rückblickend auf die Corona-Semester sowie die Zeit post Corona in den Blick genommen.

\subsection{Good Practices: Die Lehrenden als Treiber guter Hochschullehre}

In den Interviews dieser Zielgruppe kristallisierten sich verschiedene Themenbereiche als hier besonders erwähnenswert heraus (s. Abbildung 23). Dies sind größtenteils keine neuen Lehrformate, sondern an die digitale Lehre angepasste Veranstaltungen und Prüfungsformate. 


\section{Good Practices}

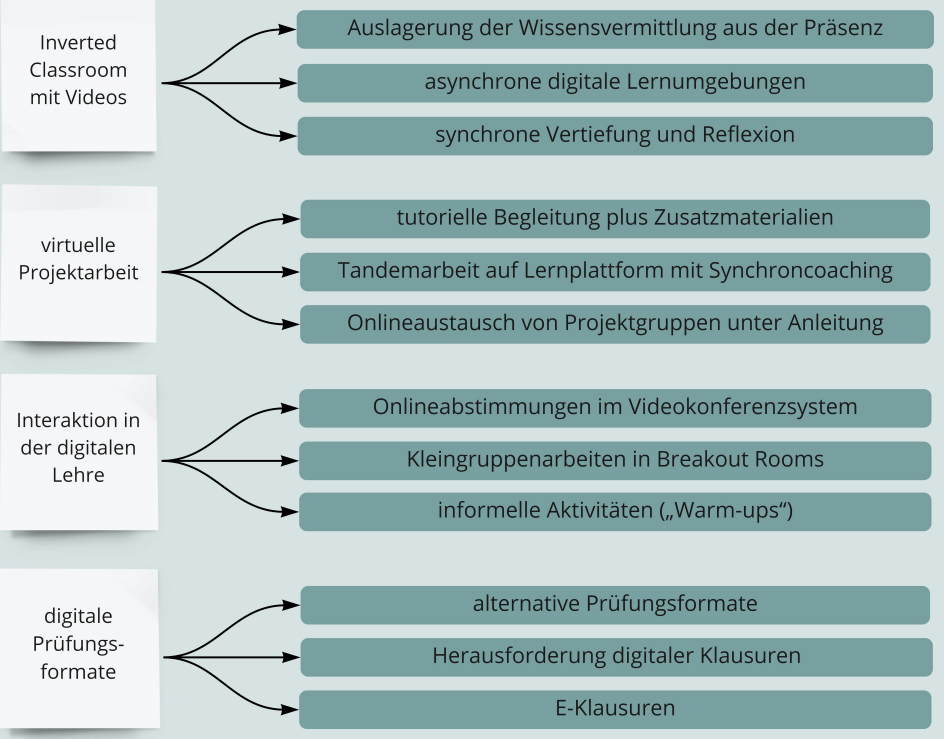

\section{Abbildung 23: Übersicht der Good Practices}

Der Inverted Classroom mit Videos, die Verschiebung der reinen Wissensvermittlung in den asynchronen Bereich vor den Veranstaltungen, um dann wertvolle Präsenzzeit für vertiefte Übungen und Diskussionen nutzen zu können (Handke \& Sperl, 2012), wurde gerade von dieser Zielgruppe der treibenden Lehrenden teils auch schon in der Präsenzehre vor der Pandemie genutzt. Anders verhält es sich bei der Methode der Projektarbeit: Diese ist ein stark praxisorientiertes Format, sodass hier die spannende Komponente vor allem in den Fragen besteht, ob und wie diese Veranstaltungsform in die digitale Lehre übertragen werden kann und wo die Grenzen liegen. Hier stellt sich die Frage, ob es für Projektarbeiten ein funktionales Äquivalent im Digitalen überhaupt gibt. Das Thema virtuelle Interaktion ist ein speziell digitales in den Corona-Semestern. Bereits in großen Präsenzgruppen gab es die Problematik des Monologs mit wenig Feedback, 
in der digitalen Lehre mit fehlender sozialer Präsenz (Bremer, 2007) tritt dieses Problem noch deutlicher zutage. Auch hier soll auf einige Good-Practice-Ansätze der befragten Lehrenden geschaut werden. Abschließend geht es um Prüfungsformate, die zu jeder Veranstaltung gehören und digital umgesetzt werden mussten.

Im Vergleich zu den bisherigen Ausführungen sollen in den folgenden Fallbeispielen Geschichten nah an der Lebensrealität erzählt werden. Darum wird im Folgenden auch ein anderer Erzählstil verwendet. Um den Lesefluss dabei nicht zu unterbrechen, sind die Geschlechter der Personen in den Fallbeschreibungen zufällig gewählt und entsprechen nicht zwangsläufig dem tatsächlichen Geschlecht.

\subsubsection{Good Practice: Inverted Classroom mit Videos}

Viele Stunden investierte er, um die wichtigsten Inhalte seiner Lehrveranstaltungen in 10- bis 15-minütigen Videos zu Hause aufzuzeichnen, damit diese den Studierenden vor den Veranstaltungen und auch zur Nachbereitung zur Verfügung stehen. Dies machten viele Kolleg*innen im Frühjahr 2020 auch, als die Onlinelehre plötzlich für alle Hochschulen die einzige Möglichkeit war. Aber dieser Professor setzte schon seit vielen Semestern auf dieses hybride Konzept mit Lehrvideos und ergänzender Präsenzveranstaltung. Bisher hatte er jedoch, wie er eingestand, auch aus Zeitgründen, nur „einzelne Elemente, keine kompletten Module“ (IL1: 6) digital umgesetzt. Diese wurden von den Studierenden gerade auch zur Prüfungsvorbereitung dankend angenommen und immer wieder angeschaut.

Als im Frühjahr 2020 dann der Wechsel von der Präsenz- in die rein digitale Lehre so abrupt erfolgte, musste er nicht bei null anfangen, sondern er konnte auf seinen bestehenden Ansätzen aufbauen. Trotzdem sagte er, er habe noch „hunderte (weitere) kleine Lernvideos aufgenommen“ (IL1: 6). In diesen Videos sprach er teils Erklärungen zu seinen Folien, simulierte zum anderen aber auch Tafelaufschriebe in Word. Diese wurden dann als kleine Lernhappen in die Lernumgebung der Hochschule hochgeladen, denn „wenn irgendwas länger als zehn Minuten dauert, dann ist es eh vorbei bei den jungen Leuten“ (IL1: 6). Jede seiner Veranstaltungen bereitete er asynchron vor und 
stellte die Lerninhalte zur Verfügung; dies auch aus Angst, dass die zeitgleiche Onlinelehre technisch nicht funktionieren könnte. In jedem seiner Module gab es für jede Woche eine Arbeitsanweisung, welche Texte und Videos die Grundlage und welche die begleitenden Übungen waren. Außerdem gab es immer den Verweis auf die entsprechenden Aufgaben in alten Klausuren. Dies alles war gebündelt in der Lernumgebung zu finden, die in allen Modulen der Ausgangspunkt für die Studierenden war, um von dort zum Beispiel auch die jeweiligen Links zu den synchronen Besprechungen abzurufen.

Ein anderer Professor hatte den Vorteil, vor dem „Muss an digitaler Lehre“ im Rahmen eines Projekts schon klassische Vorlesungsaufzeichnungen mit Tafel und Beamer und einer in den Raum gestellten Kamera erstellen zu können. Dadurch wurde schon vor Jahren versucht, die Grundlagenveranstaltungen in ein zeitlich flexibles Inverted-Classroom-Prinzip umzustellen. Er sagte dazu ganz selbstkritisch und lachte: „Wenn man das anderthalb Stunden am Stück versucht anzuschauen, ist es das beste Schlafmittel, das man sich nur vorstellen kann" (IL2: 10). Trotzdem waren auch diese Videos schon hilfreich für die Studierenden, die sie vor allem zur Prüfungsvorbereitung nutzten. Durch dieses Projekt konnte er sein Wissen zur digitalen Lehre aufbauen. Die Erarbeitung des Lernstoffs zu den Veranstaltungen erzwang er durch verpflichtende Abgaben vor der jeweiligen Veranstaltung: Studierende, die die Aufgabe nicht bearbeitet hatten, durften nicht an der jeweiligen digitalen Vorlesung teilnehmen. Er wartete wirklich jedes Mal so lange, bis diese sich aus der Videokonferenz wieder ausgeloggt hatten: „Also ich habe sie einfach, mit allerdings sehr viel Begleitung, muss man dazu sagen, an die Wand laufen lassen" (IL2: 18). Dieses Konzept funktionierte in den Grundlagenveranstaltungen für ihn gut, denn „wichtig ist, dass sie einfach sich mit den Aufgaben beschäftigen und dann halt echte Fragen haben zu dem Stoff“ (IL2: 10).

Dass man aber auch ohne zusätzliche Förderung, nur aus eigenem Antrieb und Motivation und sogar mit widrigen Rahmenbedingungen gute Onlinelehre für die Studierenden gestalten kann, zeigte das dritte Fallbeispiel: Die Professorin war von Beginn ihrer Lehrtätigkeit an davon überzeugt, dass es wichtig ist, Lerninhalte auf mehreren Kanälen, also auch asynchron, zum Beispiel in Form von Videos, zur Verfügung zu stellen, damit sich die Studierenden in ihrem eigenen 
Tempo und ihrer eigenen benötigten Intensität mit den Lerninhalten beschäftigen können. Nur die Zeit, um für alle wichtigen Inhalte selbst Lernvideos zu drehen, war nicht da. Also stellte sie offen verfügbare kurze YouTube-Videos von Kolleg*innen auf der Lernplattform der Hochschule zur Verfügung und band sie als Links in ihr Skript ein. In diesem fanden sich auch Beispiele, die dann normalerweise in der Vorlesung gemeinsam handschriftlich erarbeitet wurden. In den Corona-Semestern intensivierte sie dann ihr Engagement, um den fehlenden persönlichen Kontakt durch mehr Angebote auszugleichen: Mittlerweile stellte sie für alle wesentlichen Lerninhalte ihrer Veranstaltungen kurze Lernvideos zur Verfügung, indem sie jede Vorlesung live aufnahm und dann einzelne Teile geschnitten im Nachgang zur Verfügung stellte, was ein doppelter Arbeitsaufwand war. Durch das Gesamtskript, aus dem alle Inhalte verlinkt waren, gewann sie die Freiheit, Inhalte in der Vorlesung so schnell oder langsam zu erarbeiten, wie die jeweilige Kohorte dies benötigte, und verwandelte die Veranstaltung in einen „mini-invertierten Classroom-Teil sozusagen, also dass ich Sachen rausschiebe aus der Vorlesung, die einfach sind, und dafür mich mehr auf Fragen und auf schwierige Dinge konzentriere“ (IL3: 31). Die Aufgaben zu den Lerninhalten waren auch aus dem Skript verlinkt, sodass die Strukturierung sich von allein ergab und nur noch die Information, welche Seiten des Skripts in der nächsten Veranstaltung behandelt werden, kommuniziert werden musste.

Dadurch fällt auch in gewisser Weise die Taktung der Lerninhalte weg. Sie stellt am Anfang des Semesters alle Inhalte, die sie hat, zur Verfügung und jede $e^{\star}$ kann im eigenen Tempo das Skript, die Videos und die Aufgaben bearbeiten. In den Onlinevorlesungen wird zwar jede Woche weiterhin ein bestimmtes Thema behandelt, niemand ist jedoch gezwungen, sich an diese Taktung zu halten, und kann schneller oder auch langsamer vorangehen. Mit diesem Konzept machte sie sehr gute Erfahrungen, auch weil die begleitenden Tutorien als Sprechstunden dienten. Die Studierenden lernten im letzten Winter online besser als in den Präsenzjahren davor und sie sagte ironisch: „Eigentlich bin ich der Meinung, wir sollten unsere Studenten immer von Oktober bis Januar wegsperren“" (IL3: 51).

Insgesamt wird von einem Großteil dieser Zielgruppe der vorangehenden Lehrenden an ihren Hochschulen dieses Konzept vertreten: 
Grundlagenwissen solle nicht Semester für Semester in Präsenz als Vorlesung immer wieder gleich doziert werden, sondern zur Vorbereitung zur Verfügung gestellt werden. Auf dieses Wissen kann dann in den Veranstaltungen mehr oder weniger aufgebaut werden. Dies bedeutet natürlich auch einen Wandel der Lehrenden-Rolle, wie ein Interviewter ganz deutlich machte: „In der Transformationsphase im ersten Lockdown habe ich vor allem meine Rolle darin gesehen, so eine Art Train-the-Trainer-Funktion zu machen" (IL5: 4).

Trotzdem hatte unser Professor aus dem erstgenannten Beispiel sein Konzept aus dem Frühjahr 2020 doch ein wenig umgestellt. Die Videos in den Modulen sind jetzt größtenteils nur als Ergänzung und zur Wiederholung gedacht und er präsentiert die Modulinhalte in den „Live-Vorlesungen“. Sein Ziel ist es, für die Zukunft dieses System wirklich für alle seine Module anzubieten. Denn seiner Überzeugung nach macht es die Mischung. Die Studierenden der Präsenzhochschulen sei nicht selbstverständlich dazu geeignet bzw. geneigt, komplett im Inverted Classroom $\mathrm{zu}$ arbeiten, also alle Inhalte vorzubereiten und dann in den synchronen Anteilen tiefgehende Informationen zu präsentieren und in die Interaktion zu kommen.

Wie konsequent dieser Rollenwechsel also auch online vollzogen wird, hängt zum einen vom intendierten Grad des Inverted-Classroom-Modells, zum anderen aber auch stark von den Vorerfahrungen der Studierenden und letztendlich von der Konsequenz und dem didaktischen Geschick des/der Lehrenden ab.

\subsubsection{Good Practice: Virtuelle Projektarbeit}

Die Projektarbeit ist gerade an den sehr praxisorientierten HAWs ein gängiges Veranstaltungsformat, zu dem es schon vielerlei Erfahrung gibt. Im Zuge der Corona-Semester wurden die Lehrenden jedoch jetzt vor die Herausforderung gestellt, dieses sehr auf Gruppenarbeit und Interaktion abzielende Format in den Onlinebereich zu übertragen. Dies fiel einigen Lehrenden leichter als anderen.

Bei einem Interview entspann sich gleich am Anfang ein reges Gespräch zum Thema innovative Lehrkonzepte, da der ältere Professor 
schon auf viel Lebens- und Lehrerfahrung zurückblicken konnte. Für ihn waren Onlinekonzepte schon ein alter Hut, da er schon seit Jahren in internationalen Netzwerken Lehrveranstaltungen durchführte, die meistens ein hybrides Format hatten. Die deutschen Studierenden waren vor Ort und internationale Teilnehmer wurden online dazu geschaltet. Daneben wurde „ein Onlinekurs aufgesetzt, der parallel lief und der eine Projektwoche vorbereitet hat" (IL4: 3). Im letzten Frühjahr mussten diese Formate dann „einfach“ auf rein digitale Veranstaltungen umgestellt werden. Da es sich hierbei um eine praxisorientierte Projektwoche handelte, war dies trotz der digitalen Vorerfahrungen doch nicht so trivial. Statt der Reisen an die Orte wurden Tutoren aus früheren Veranstaltungen akquiriert, die dann von ihren Erfahrungen berichteten und die neuen Studierenden auch inhaltlich begleiteten, „dass also das Erlebnis vor Ort zumindest teilweise ersetzt werden kann“ (IL4: 3). Die internationalen Studierenden „die da vor Ort sind und beteiligt waren, die sind hingegangen, haben mit Handykamera Filme aufgenommen, haben die dann hier eingestellt oder bzw. auch gezeigt während der Workshops" (IL4: 3). Außerdem wurden viel mehr Begleitmaterial und Hintergrundinformationen als zuvor in eine zentrale Plattform eingestellt. Von den Ergebnissen der Gruppenarbeiten war er sehr begeistert. Diese waren in den Corona-Semestern besser ausgefallen als vorher und er kannte auch den Grund: „Also ich glaube schon, dass das einen wesentlichen Input gegeben hat, sodass fast die Wahrnehmung konzentrierter war, als wenn man vor Ort ist und erschlagen wird von der Vielfalt der Eindrücke, ja?" (IL4: 3). Sind diese Projektwochen im Ausland also gar nicht mehr notwendig, auch wenn sie wieder möglich werden? Lachend schüttelte der Professor den Kopf und betonte, dass die Vor-Ort-Erfahrungen natürlich einfach nicht ersetzbar sind und als wesentliches Element zum Lernerfolg beitragen.

Dies sah auch der Lehrende im zweiten Beispiel so. Er war außerdem beim Lehrformat der Projektarbeit überzeugt, „dass eben Studierende, wenn sie im Tandem arbeiten, auch mehr und intensiver an einem Thema arbeiten, wie wenn sie das Gefühl haben: Ich bin hier totaler Einzelkämpfer" (IL5: 16). Die Lernerfahrung war durch die Praxisorientierung und die notwendige Teamarbeit intensiver als bei anderen Lehrformaten. Als Vorleistung gab es allerdings einen Test in der 
Lernumgebung der Hochschule, der bestanden werden musste. Hier gab es drei Versuche und dazwischen eine Sperrzeit von 24 Stunden, damit die Studierenden sich nach der automatisierten individuellen Rückmeldung, welche Inhalte noch mal wiederholt werden sollten, auch wirklich noch einmal mit dem Lernstoff beschäftigten. Denn das Lernen wurde durch die digitalen Anteile und die Einforderung von Feedback in Form von kleinen Übungen transparenter und die Annahme, Studierende würden sich mit den digitalen Tools leichter tun als andere, wollte er nicht unterschreiben: „Ich glaube, wir müssen uns hoffentlich jetzt endgültig von diesem blöden Mythos der Digital Natives verabschieden" (IL5: 24). Eine weitere unbenotete, zweiseitige reflexive Ausarbeitung war danach auch noch der Tandemarbeit vorgeschaltet, um alle Studierenden auf das gleiche Niveau zu bekommen. Die Projektarbeit mit Datenerhebung, -interpretation und -veröffentlichung fand dann nach diesen theoretischen Grundlagen statt und wurde vom Professor synchron und in Coaching-Situationen begleitet.

Ähnlich arbeitete der Lehrende in unserem dritten Beispiel, indem er versuchte, soziale Elemente, wie den Austausch der Projektgruppen untereinander, angeleitet in den Onlineraum zu verschieben. Die Gruppen waren aufgefordert, sich einmal die Woche zur gegenseitigen Präsentation des Projektstandes in den jeweiligen Gruppen online zu treffen und sich auszutauschen: „die zufälligen Begegnungen und der zufällige Austausch, der ist sehr, sehr reduziert und das versuche ich, durch diese etwas informelleren Treffen zumindest abzufedern" (IL8: 48). Dies zeigt auch die Grenzen diese Lehrformats: Projektarbeit lässt sich nur zu Teilen in rein digitaler Form übersetzen. Alle Lehrenden wollen jedoch die Vorteile der medialen Aufbereitung und Bereitstellung von Inhalten zur Vorbereitung und Begleitung auch fortführen, wenn die gemeinsame Arbeit an Projekten in Präsenz wieder möglich ist.

\subsubsection{Good Practice: Interaktion in der Onlineveranstaltung}

Eigentlich hatte die Professorin es schon fast aufgegeben, in großen Gruppen durch digitale Werkzeuge auch in den Präsenzveranstaltungen die Interaktion zu intensivieren. Denn der Versuch, mit dem Ein- 
satz einer App zum Fragenstellen zu animieren oder auch mit einem Abstimmungssystem zu arbeiten, hatte leider nur mäßigen Erfolg. Die gleichen schlechten Erfahrungen mit fehlender Interaktion bei großen Studierendengruppen setzten sich dann auch online fort. „Da traut sich keiner, eine Frage zu stellen“ (IL3: 37), auch wenn digitale Abstimmungswerkzeuge eingesetzt wurden, war die Teilnahme gering. Im Videokonferenzsystem, das an ihrer Hochschule genutzt wurde, war es jedoch möglich, anonym Fragen zu stellen, weil sich keiner mit dem Echtnamen anmelden musste. Zu ihrer eigenen Überraschung senkte dies die Hemmschwelle und die Studierenden trauten sich.

Einen anderen Weg ging eine andere interviewte Lehrende. In ihren Veranstaltungen versuchte sie von Anfang an, durch Kleingruppenarbeit in Breakout Rooms auch online sehr viel Interaktion herzustellen. Diese technische Hürde von Untergruppen in Videokonferenzen übte sie vorher sogar mit ihrer eigenen Familie, um Sicherheit zu gewinnen. Und so funktionierte es dann auch mit den Studierenden: „Kamera an, interaktiv, ein gutes, gutes Miteinanderarbeiten“ (IL7: 22). Ihre Persönlichkeit, die auch in der Präsenzlehre im Vordergrund stand und mit der sie die Studierenden bei der Stange hielt, versuchte sie, ins Digitale zu übertragen. Und dies war durch Interaktion und einen Methodenmix - „es gibt Inputphasen von mir und dann gibt es mal Gruppenarbeiten und auch Reflexionsmomente" (IL7: 26) - auch gut gelungen. Die Studierenden wussten, dass sie involviert wurden, und die Gefahr des Abdriftens wurde dadurch geringer. Dies geschah nicht nur durch gezwungene Abfragen oder Gruppenarbeiten, sondern vor allem durch Kleinigkeiten wie Running Gags, die sich durch die Veranstaltungen zogen, indem sie bestimmte Studierende immer wieder zu demselben Thema als „Experten” befragte oder einfach Texte auch mal vorlesen ließ. Dies war niedrigschwellig, bezog die Studierenden jedoch aktiv mit ein. Die Rückmeldungen der meisten Studierenden waren sehr positiv, doch gerade bei den Erstsemestern war bei einigen die Hemmschwelle zur Onlinekommunikation doch recht hoch: „Und dann gab es echt welche, bei denen man das Gefühl hatte: Sobald man den Namen nur anfängt auszusprechen, drücken sie auf „ich verlasse den Raum" (IL7: 22). Aber so aktiv die Studierenden auch mitarbeiteten, blieb für sie ein Rätsel, warum viele Studierende so eine Scheu davor hatten, die Kamera anzumachen, und forderte die 
visuelle Präsenz vehement ein: „Sie wissen, ich rede nicht mit den schwarzen Löchern. Bitte machen Sie die Kamera an" (IL7: 40). Für sie war dieser Blickkontakt wichtig und half bei ihrem Unterrichtsszenario, trotzdem erreichte sie gerade bei größeren Gruppen nicht alle damit. Einen großen Vorteil der virtuellen Kleingruppenarbeit, bei der sie online automatisch immer wieder andere Studierende zusammenwürfeln konnte, sah sie: „mischt sich das besser, also zwangsweise, finde ich gut" (IL7: 60). Dafür suchte sie noch eine gute Lösung in der Präsenz, da sich dort fast immer die gleichen Gruppen zusammenfanden.

Anders herum gestaltete es sich im folgenden Beispiel: Für Dinge, die der Professor in der Präsenz schon als Methodenmix einsetzte, musste er online nach neuen Lösungen suchen. Auch er nutzte dafür sehr gern die Breakout Rooms: „Ich mache zum Start gerne so ein kleines Warmup, irgendwas Informelles, was so lose mit dem Thema des Unterrichts gekoppelt ist" (IL8: 32), oder auch eine Bewegungsübung als Pause zwischendurch. Dies war für ihn gar nicht „typisch online“ (IL8: 34), ließ sich im digitalen Raum aber seiner Meinung nach auch leicht umsetzen. Diese Angebote waren jedoch freiwillig für die Studierenden und es war ihm generell wichtig, immer wieder die Rückmeldung auch zu seinen Lerninhalten bei den Studierenden abzuholen: „Und dann mache ich periodisch, vielleicht jede dritte, vierte Veranstaltung, am Ende so ein kurzes I like, I wish, eine kurze Abfrage, was gut war und was noch an Wünschen offen ist" (IL8: 36).

Und auch wenn die Rahmenbedingungen an vielen Hochschulen und für die meisten Veranstaltungen so nicht gegeben sind, soll als Ausblick das Beispiel eines Professors, der die Angebote seiner freiberuflichen Tätigkeit nutzte, um seine Veranstaltungen in der Hochschule ansprechender und interaktiver zu gestalten, hier den Abschluss bilden. Er nutzte eine VR-Software, die echte Räume und eine Zusammenkunft mit Avataren simulierte. Die soziale Komponente war dadurch sehr viel höher, da sich durch die auf die Lerninhalte speziell auszurichtende Gestaltung des Kursraums, das anpassbare Layout und die Auswahl der Avatare Individualität simulieren ließ. Es entstand dabei ein haptisches Gefühl, wenn mit einem Avatar die Treppe hinaufgegangen werden musste, um in den Raum für die Gruppenarbeit zu gelangen. Nach den positiven Erfahrungen im ersten Onlinesemester entstand daraus eine Mini-Studie, indem er für eine seiner Veranstal- 
tungen das Standard-Videokonferenztool seiner Hochschule und für eine andere die VR-Software nutzte. Beiden wurde der Zugang zu den Gruppenarbeitsräumen in der VR-Software ermöglicht. Das Resultat war, dass die VR-Gruppe insgesamt mehr Interaktion auch in den Vorlesungen zeigte und die Gruppenräume auch außerhalb der Veranstaltungszeiten sehr viel intensiver nutzte. Dies erklärte er sich zum einen mit dem besseren Austausch in der Vorlesung und einem dadurch von Anfang an engeren Vertrauensverhältnis untereinander, zum anderen ergab sich bei der VR-Gruppe kein Medienbruch zur Gruppenarbeit, da das gleiche Tool genutzt wurde.

\subsubsection{Good Practice: Digitale Prüfungsformate}

Alle Lehrformate und didaktischen Elemente, die für Veranstaltungen gewählt werden, zielen immer auf die Erreichung der Lehr- und Lernziele und damit das Bestehen des jeweiligen Moduls ab. Deshalb ist es unabdingbar, den Studierenden eine Prüfungsleistung abzuverlangen, um diese bewerten zu können. Unabhängig vom digitalen Setting stellen sich die Lehrenden dabei die Frage, ob vor allem Wissen, zum Beispiel in Grundlagenfächern, abgefragt wird oder es um Kompetenzen und Wissenstransfer geht. Je nachdem ist es geboten, verschiedene Prüfungsformate zu wählen (Biggs \& Tang, 2011).

Eine Professorin, die in eher projektbezogenen Modulen, besonders auch im Masterstudiengang, mit ganz anderen Lehrkonzepten, viel Gruppenarbeit und fallbezogenen Prüfungsformen arbeitete, fand es schwierig, in einem Grundlagenfach eine andere Prüfungsform als Wissensabfragen mit Klausuren zu nutzen. In einem Grundlagenfach zum Studienbeginn müssen einfach gewisse Fakten abgeprüft werden: „Wenn ich von diesen Aufgaben weggehen würde, dann wäre ich schon fast da, wo man in einem Mathematikstudium ist, nämlich auf Verständnis und auf Theorie zu prüfen. Und ich glaube, damit tue ich weder mir noch meinen Studenten einen Gefallen" (IL3: 61). Das Gleiche galt natürlich jetzt bei den digitalen Prüfungen. Hier gab es auch Lehrende, die sowohl durch die geltenden Prüfungsordnungen als auch durch die bürokratischen Strukturen einen großen „Beharrungsdruck“ (IL5: 8) spürten. Ein Interviewter stellte jedoch auch 
ganz klar fest, dass es viele Kolleg*innen gab, bei denen die Transformation „from teaching to learning“ (IL5: 8) auch ganz unabhängig von den Onlineformaten nicht ankam und „auch das Ausprobieren von neuen Prüfungsformaten schlicht und einfach nicht vorstellbar ist" (IL5: 8). Das sollte ihn eigentlich erst mal nicht stören, da er selbst keine Klausuren in seinen Veranstaltungen stellte. Er nutzte in den Veranstaltungen vor allem Projektarbeiten als Prüfungsformat, die aber auch im Tandem geschrieben werden konnten. Dies war auch in der Prüfungsordnung so abgebildet. An der Hochschule war es jedoch Usus, dass die Prüfungsaufsichten unter allen Lehrenden gerecht aufgeteilt wurden. Und so musste er auch Prüfungsaufsichten in Präsenz ableisten, obwohl er selbst, gerade in diesen Pandemiezeiten, andere Prüfungsformen wählte: „Ich fand es schade, dass es nicht als Chance gesehen wurde, sondern eher als Bedrohung" (IL5: 8).

Ein anderer Lehrender schmunzelte bei der Frage, wie denn die Prüfungen in den digitalen Semestern gelaufen seien. Die Problematik, Klausuren im digitalen Raum abbilden zu wollen oder zu müssen, hatte er nicht: „Ich hatte schon vorher keine Klausuren, bevor es mit Corona losging, in meinem Prüfungsportfolio. Insofern war ich an der Stelle persönlich ein bisschen raus. Das war ganz gut" (IL4: 5). Schriftliche Ausarbeitungen, die er unter anderem nutzte, waren unabhängig davon, ob die Lehre in Präsenz oder virtuell stattfand:

Also ich lasse nur über Studienarbeiten, Präsentationen, schriftliche Arbeiten, was auch immer, da gibt es unterschiedliche Dinge, ich habe verschiedene Formate da entwickelt, Case Studies, also wo die dann so Best Practices recherchieren und dokumentieren und Learnings ableiten, also gibt es ganz verschiedene Dinge, die die machen müssen oder können. (IL4: 5)

Mit diesen alternativen Prüfungsformaten umgingen diese Lehrenden die Problematik der Rechtssicherheit von Take-home-Exams oder Open-book-Klausuren, die zu Hause durchgeführt wurden. Denn hier konnte nicht sicher überprüft werden, ob diese Prüfungen wirklich von dem/der Studierenden abgelegt wurden:

Also das ist in meinen Augen tatsächlich die Hauptschwierigkeit im Moment in dieser Corona-Situation und dem digitalen Unterricht, die Prüfungen hinzubekommen, dass sie rechtssicher und guten Gewissens 
durchgeführt werden können, auch bei Lehrveranstaltungen, wo eben tatsächlich eine schriftliche Prüfung angemessen ist. (IL2: 64)

Und die treibenden Lehrenden dachten auch noch weiter: Prüfungen und insbesondere Klausuren ließen sich digital ja nicht nur zu Hause umsetzen. Sogenannte E-Klausuren, die auch in Zeiten vor Corona gerade an großen Universitäten schon Einzug gehalten hatten, indem in großen Computerräumen die Klausur unter Aufsicht digital erstellt wurde, wünschten sich auch einige Lehrende für die kleineren HAWs. Hier wurde jedoch das Problem fehlender Räumlichkeiten gesehen, in denen digitalisierte Prüfungen einigermaßen rechtssicher abgenommen werden konnten, sodass „man jetzt nicht den Leuten die Wirbelsäule festschrauben muss, weil sie rechts und links eben den Monitor des Nachbarn sehen“ (IL2: 64). Die Kohorten sind gerade zu Studienbeginn in den Grundlagenfächern teilweise so groß, dass es längst nicht genügend Computerarbeitsplätze geben würde: „Wir könnten ja E-Klausuren stellen und die dann überwacht irgendwo in einem Computerraum nehmen. Aber haben Sie gehört, was ich für Massen habe. So viele Computerarbeitsplätze haben wir gar nicht“ (IL3: 63).

Stellten auf der einen Seite die Konzeptionalisierung und Durchführung digitaler Prüfungen eine Herausforderung für die Lehrenden dar, berichteten diese auf der anderen Seite von positiven Erfahrungen in Hinblick auf den Lernerfolg der Studierenden in den beiden Corona-Semestern. Die Prüfungsleistungen seien „vom Schnitt her deutlich besser als vor Corona” (IL1: 4). In seinem Modul, so ein weiterer Professor, sei bei der Klausur ein deutlich besserer Schnitt herausgekommen als in der Vergangenheit, obwohl er „die Klausur nicht einfacher gemacht habe" (IL1: 6), und das habe sich auch im zweiten digitalen Semester erneut bestätigt.

\subsection{Die Zukunft digitaler Lehre aus der Sicht der treibenden Lehrenden}

Die Digitalisierung wurde pandemiebedingt zwangsläufig zur Normalität in weiten Teilen des Hochschulalltags. Auf didaktischer Ebene wurden Lehrsettings und Klausuren umgestellt, auf technischer Ebene mussten für die Gestaltung der digitalisierten Lehre Lösungen gefunden werden und nicht zuletzt veränderten sich mittels digitaler 
Werkzeuge maßgeblich die Kommunikationsformen. Aus verschiedenen Perspektiven wurde in den Fallbeispielen aufgezeigt, wie Lehrende, die aufgrund von Vorerfahrungen mit digitaler Lehre eine treibende Funktion im digitalen Wandel an ihren Hochschulen hatten, mit der Umstellung auf ein digitales Semester im Frühling 2020 umgingen. Dabei wurden Ansätze fokussiert, die sich in der konkreten Lehrsituation als Good Practices bewährten. Im letzten Teil von Kapitel 6 wird nun das Hauptaugenmerk auf die zweite forschungsleitende Teilfrage gelegt: Welche Chancen und Wünsche für die digitale Hochschulehre lassen sich aus diesen Erfahrungen ableiten?

\subsubsection{Chancen digitaler Lehre}

Es ist anzunehmen, dass die treibenden Lehrenden mit ihren Praxisansätzen nicht nur aus struktureller Sicht eine solide Grundlage schufen, um die Digitalisierung der Lehre institutionell in die Breite zu tragen, sondern auch durch ihr Engagement und die Gestaltung von Good Practices in ihrer Lehre einen wesentlichen Beitrag für die Verankerung einer positiven digitalen Lernkultur bei ihren Studierenden leisteten. Aus diesem Grund wurde in der Analyse der Interviews besonders aufmerksamkeitsstark darauf geblickt, welche Erfahrungen in der Arbeit mit digitalen Lehrsettings Potenzial hinsichtlich einer weniger gezwungenen, eher erstrebenswerten digitalen Normalität zeigten. Folgende didaktische Aspekte waren dabei wesentlich (s. Abbildung 24):

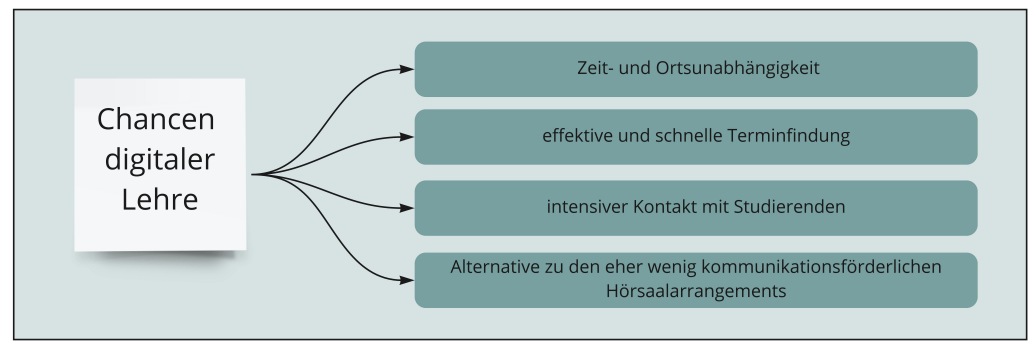

Abbildung 24: Übersicht der Chancen digitaler Lehre 
Die Chancen digitaler Lehre im Zusammenhang mit den damit einhergehenden, teils bisher noch unbekannten Freiheiten im Studium und den neu gewonnenen Flexibilisierungen in der Lehre wurden in der vorliegenden Studie bereits mehrfach erwähnt. Einleitend sollen dennoch diese Aspekte auch in diesem Kapitel zusammenfassend aufgegriffen werden, eine etwaige Redundanz zu den anderen Zugängen stellt sich in dem Fall nicht nur anhand der durchweg erkannten Relevanz dieser Faktoren für neue Gestaltungsmöglichkeiten von Lehre, sondern auch durch den hier gelegten Fokus auf die erprobten GoodPractice-Erfahrungen als berechtigt dar. Die Auslagerung der Inhalte aus der Präsenzvorlesung in asynchrone Module (s. Beispiele zu Inverted Classroom mit Videos in Kapitel 6.2.1) führte dazu, dass die Studierenden durch die Entschärfung des strengen Semesterplanablaufs neue Möglichkeiten hatten, sich mit dem Lernangebot in selbstgewählter Progression und Intensität zu beschäftigen. Der Mehrwert der Zeitund Ortsunabhängigkeit, die mit den Onlineveranstaltungen verbunden war, wurde ebenfalls in der Beschreibung von Good Practices hervorgehoben. So wurde die Möglichkeit, Livevorlesungen so anzubieten, dass Studierende diese von zu Hause aus im Livestream mitverfolgen konnten, aus verschiedenen Gründen - „wenn jemand krank ist, auch aus Verkehrsgründen oder Umweltgründen” (IL3: 91) - als Vorteil betrachtet. Insbesondere im Kontext einer „Pendler-Hochschule“ (IL4: 37) war durch die flexiblere Gestaltung der Onlineveranstaltungen von „deutlich höheren Beteiligungszahlen“ (IL4: 37) an Vorlesungen die Rede. Ferner wurden auch vor dem Hintergrund einer besseren Ressourcennutzung Vorteile in der digitalen Lehrsituation erkannt, unter anderem in puncto Workload und Zeitbudget, „weil eben Anfahrtsund Abfahrtszeiten wegfallen“ (IL5: 2). Die Überbrückung der räumlichen Distanz über Videokonferenztools zog zudem den Vorteil einer effektiveren und schnelleren Terminfindung nach sich: „Es ist sehr viel einfacher, kurzfristig zu reagieren und mal ein Gespräch mit einer Gruppe zu organisieren. Das war völlig unmöglich vorher, hätte ich zumindest gedacht, also es gab dafür gar keine Infrastruktur, um kurzfristig eine Rücksprache zu halten“ (IL8: 42). Über das Videokonferenztool war die Kommunikation mit den Studierenden in der Regel niederschwelliger, diese hatten „das Gefühl, dass sie einen [die Lehrenden] schneller erreichen“ (IL7: 16) konnten, des Weiteren 
wurde der Kontakt mit den Studierenden dadurch intensiviert, dass das Sprechstundenangebot häufiger als zuvor in Anspruch genommen wurde. Die Mehrbeteiligung an virtuellen Sprechstunden ließ sich zusätzlich auf eine differenzierte Betreuungsqualität zurückführen, denn das Digitale schuf die Möglichkeit einer Eins-zu-eins-Betreuung, die davor in Präsenz weder in der Form noch in dieser Effizienz stattfand. Zudem ergab sich ein spannender Nebeneffekt: Die Onlineveranstaltungen wurden als eine perspektivische Alternative zu den eher wenig kommunikationsförderlichen Hörsaalarrangements bezüglich des Austauschs zwischen den Studierenden betrachtet: „Das finde ich im Digitalen faszinierend, dass ich, wenn die Kameras an sind, dann alle sehe. Aber sie sehen sich auch gegenseitig. Und das finde ich super, dass diese Hierarchisierung rausgenommen wird, die ja manifest [ist] in manchen Hörsaalstrukturen" (IL5: 28).

\subsubsection{Wünsche für die digitale Lehre in Zukunft}

Die Wünsche und Vorstellungen, die für die Weiterentwicklung guter Ansätze in der Hochschullehre herausgearbeitet wurden, werden im Folgenden mit Fokus auf die didaktisch relevanten Aspekte näher beleuchtet (s. Abbildung 25).

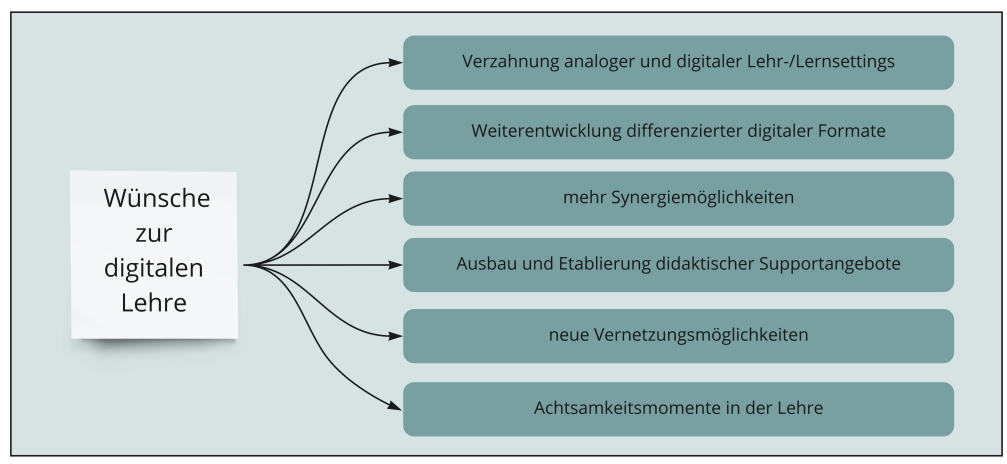

Abbildung 25: Wünsche zur digitalen Lehre 
Aus dem Einblick in die Vorstellungen guter digitaler Lehrszenarien und Wünsche für die künftige Hochschullehre erschloss sich ein umfassendes und facettenreiches Bild. Das Anliegen, nach Corona die Verzahnung analoger und digitaler Lehr-/Lernsettings beizubehalten, wurde allgemein konstatiert. Konkret tauchte dieser Aspekt stark im Zusammenhang mit hybriden Konzepten auf, die sich in der praktischen Umsetzung als geeignet erwiesen und das Potenzial $\mathrm{zu}$ einer differenzierten Weiterentwicklung aufzeigten. Die befragten Lehrenden stützten sich in den Interviews auf die Perspektive, dass die Einbindung digitaler Werkzeuge in die Hochschullehre sowie die Gestaltung hybrider Veranstaltungen zukünftig zur Normalität werden:

Bleiben werden die Werkzeuge, die wir jetzt so verwenden, sowie andere Werkzeuge auch und dass es nichts Besonderes mehr ist, wenn man sagt: Okay, wir machen jetzt eine hybride Veranstaltung, wir switchen, wir machen einen Flipped Classroom. Das wird ein ganz normales Toolset sein. (IL5: 28)

Die Verzahnung digitaler und analoger Lernumgebungen wurde in einem Interview mit einer „Güterabwägung“ (IL4: 37) verglichen, dabei richtete sich die Aufmerksamkeit auf eine neue Dimension von hybridem Lernen, die insofern Realität werden kann, dass die Lehrenden künftig Vor- und Nachteile abwägen und bereits erprobte digitale Settings ausbauen können, auch - oder vor allem - wenn sie nicht mehr dazu gezwungen sind. Betont wurde die entscheidende Rolle einer solchen Abwägung für die „Nutzung von Know-how, das sich jetzt ansammelte“ (IL3: 89) und die „Vielfalt der Ansätze, die Hochschule ausmachen“ (IL3: 90). Aus der Überzeugung heraus, „wir werden beides behalten, auch wenn es immer wieder die Rufe nach „Wir wollen zurück in die Normalität“ gibt“ (IL5: 4), wurde hier im Interview die Haltung ersichtlich, dass das Digitale bleiben wird, insbesondere „weil es eben einfach viele Vorteile hat" (IL5: 4). Aus dem Blickwinkel der treibenden Lehrenden kristallisierte sich also mehrheitlich heraus, dass sich die nächste Innovationswelle durch eine Wertschätzung definieren wird zwischen dem, was nur im Analogen möglich ist, und dem, was man davon im Digitalen abbilden kann, mit dem Ziel, „eine Balance aus beiden Welten zu finden“ (IL5: 4).

Eng gekoppelt an diese Vorstellung hybrider Modelle nahm unter den geäußerten Wünschen für die Zukunftslehre die Weiterentwicklung 
differenzierter digitaler Formate einen bedeutenden Stellenwert ein. Aus den Interviews ging hervor, dass die bereits entwickelten digitalen Formate und Materialien möglichst eine Weiterverwendung finden sollen, zumal sie sich aus den Erfahrungen in den Corona-Semestern heraus als probate Unterstützung - „zu Hause oder in der Straßenbahn“ (IL3: 89) - entpuppten und diesbezüglich auch post Corona einen didaktischen Mehrwert bieten können: „Alles, was man jetzt erstellt, [wird] weiterbenutzt und wieder zurück im Präsenzunterricht, wird das nicht nur den Präsenzunterricht verändern, sondern es wird auch die Qualität der Lehre noch mal intensiver machen oder die Betreuungsintensität, die wir bieten können“ (IL3: 89).

Eine nachhaltige und effiziente Nutzung digitaler Ressourcen betreffend ließen die Interviewergebnisse Rückschlüsse auf einen starken Wunsch nach mehr Synergiemöglichkeiten zu. Diese wurden in mehreren Zusammenhängen formuliert, unter anderem in der Vorstellung einer landesweiten, leistungsfähigen gemeinsamen Plattform zur lehrbezogenen Kommunikation und zum Datenaustausch:

Und ich würde mir an den Stellen wünschen, dass es da eine gemeinsame große, zumindest mal eine Baden-Württemberg-Plattform gibt, die leistungsfähig ist, die professionell ist und die auf einem ganz anderen Level arbeiten kann, wenn man diese Synergien mal schafft. Also das wird wahrscheinlich aufgrund der ganzen Machtzentren und Ressourcen, auf denen man dann gerne sitzt usw., schwierig sein, aber das ist ein Punkt, den verstehe ich null. Ja, das würde ich mir an der Stelle mal gerne strukturell wünschen, da wären wir sehr viel leistungsfähiger und auch als Stimme viel, viel kräftiger in anderen Kontexten. (IL4: 45)

Genannt wurden in dem Zusammenhang weitere Vorteile einer KoEntwicklung von Infrastrukturen und Bildungskonzepten, darunter allgemein, dass man innerhalb einer Hochschulstruktur „die Unterrichtsvorbereitung für eine viel größere Zielgruppe machen ... und dafür den Aufwand teilen [könnte]“ (IL8: 78), aber auch im Rahmen eines konkreten didaktischen Praxisansatzes wurde dieses Wunschbild geschildert:

Also wenn ich jetzt ein Thema habe, sagen wir Interviews mit Nutzern, könnte man nicht die vielleicht spannendste Person im deutschsprachigen Raum dafür gewinnen, mit der Person ein langes Gespräch führen, das zu einem guten Beitrag zusammenzuschneiden, aber dann eben deutschlandweit in allen Gestaltungshochschulen nutzen? Also ich glaube, in so 
eine Richtung gibt es da auch ein Potenzial in der Digitalisierung, einen Aufwand betreiben zu können, gerechtfertigt dadurch, dass man es dann auch mehr als einmal nutzt, also sowohl über mehrere Hochschulen hinweg als auch mehrere Semester hinweg. (IL8: 78)

Hieran schließt sich auch das Anliegen, durch die digitale (ortsunabhängige) Einbindung von Gastlehrenden oder aufgezeichneten Vorträgen in die Vorlesung neue Vernetzungsmöglichkeiten zu schaffen oder vertiefend aufzubauen. Darauf bezugnehmend wurde in einem Interview betont, dass es „teilweise sehr viel leichter [ist], mal irgendwie einen Gastdozenten zu Veranstaltungen dazu zu bekommen“ (IL2: 60), und dieses auch später, nach der Pandemie, weiterhin beibehalten wird: „Ja, dass wir die Leute eher online dazu holen“" (IL2: 60).

Didaktische Supportangebote, beispielsweise regelmäßige Tutorials oder kontinuierliche Ansprachen, wurden in den beiden Corona-Semestern als impulsgebende und unterstützende Formate empfunden, die dazu führten, „dass man da immer wieder am Ball bleibt“ (IL7: 56). Erwähnt wurde auch der Mehrwert digitaler Arbeitsgruppen, die sich über unterschiedliche Lehrformate und erprobte Methoden fakultätsintern sowie hochschulübergreifend austauschten. Diese Schulungen und Beratungen auszubauen und $\mathrm{zu}$ etablieren, wurde von einigen Lehrenden als Wunsch formuliert, damit die teils neu erworbenen Kompetenzen weder stagnieren noch versickern. Im Anschluss daran wurde auch der Wunsch adressiert, „dass auch die Politik erkennt, dass wir hier sehr viel mehr Medienkompetenz und IT-Kompetenz brauchen" (IL5: 26), was auch bedeutet, dass Hochschulen die Förderung dieser Kompetenzen aktiv anbieten.

Für die Möglichkeiten von Hochschullehre in der Zukunft soll hier abschließend ein letzter Gedanke angeführt werden, der nicht nur in einem Interview zur Sprache kam. Konkret wurde dabei das Anliegen formuliert, durch mehr Achtsamkeitsmomente den Menschen in den Mittelpunkt der Lehre zu stellen. Hingewiesen wurde in dem Zusammenhang auf Angebote, die für Gruppen angeboten werden können, in der Annahme: „Wenn man die Menschen auch immer mal in den Fokus bringt, können die auch leichter gerade digital arbeiten“ (IL7: 72). Damit man aber als Lehrende* $r$ dann nicht „an der eigenen Hochschule irgendwie als seltsamer Typ dasteht, wenn [man sich] da Gedanken über das Thema der Achtsamkeit mach[t]“ 
(IL5: 8), wurde der Wunsch nach mehr Unterstützung geäußert, denn das Achtsamkeitsthema sei letzten Endes auch „eine Kulturfrage, die von der Hochschule adressiert werden muss und sollte“. 


\section{Leitmotive und Handlungsimpulse für eine Pfadentwicklung nach der Corona-Pandemie}

In Kapitel 7 stehen die Zusammenführung und Interpretation der Ergebnisse aus dem Kapitel 4, Kapitel 5 und Kapitel 6 sowie deren Überführung in Handlungsimpulse im Zentrum. Zunächst werden die Ergebnisse in einen Kontext gestellt (s. Kapitel 7.1). Anschließend werden Leitmotive und Handlungsempfehlungen in den Dimensionen Akteur*innen (s. Kapitel 7.2 und Kapitel 7.3), Strukturation (s. Kapitel 7.4 und Kapitel 7.5) sowie Strategie (s. Kapitel 7.6 und Kapitel 7.7) diskutiert.

\subsection{Theoretische Einbettung und Kontextualisierung}

Die vorangegangenen Kapitel haben bereits einen detaillierten Überblick über die Ergebnisse der Studie bezogen auf die einzelnen Zugänge geliefert.

In den Interviews mit den Hochschulleitungen (s. Kapitel 4) wurde diskutiert, dass gemeinsam erreicht wurde, im Möglichkeitsraum Struktur durch Handeln zu erobern. Innerhalb des Pandemiegeschehens wurde sichtbar, dass die zunächst strukturlose Situation innerhalb der Kontingenz dazu führte, dass Akteur*innen experimentell und iterativ mit der Situation umgegangen sind. Durch die Beschreibung in den Interviews wurde klar, dass viele Lösungen und Herangehensweisen entworfen wurden. Vielfach zeigte sich auch, dass bestimmte Vorgehen und Strukturen lediglich Übergangslösungen sein können. An manchen Stellen zeichneten sich aber auch schon erste strategische Gedanken ab.

Dass innerhalb der Corona-Semester nicht nur reagiert, sondern auch agiert und innovativ vorangedacht wurde, zeigten viele Beispiele in Zugang 2 (s. Kapitel 5) und Zugang 3 (s. Kapitel 6). Die Forschungsfrage, wie der Kontingenzraum gestaltet wurde, wurde aus der Per- 
spektive untersucht, welche Möglichkeiten und Notwendigkeiten aus der jeweiligen Situation hervorgingen. Die Fokusgruppen mit Lehrenden und Studierenden sowie die Experteninterviews mit treibenden Lehrenden lieferten dabei wertvolle Einblicke in die Gestaltung der konkreten Lehr-/Lernsituation und Gesamtsituation.

Die in Abbildung 26 dargestellte Bandbreite des Kontingenzraums (hier durch die beiden horizontalen Pfeile dargestellt; s. auch Kapitel 1) bedingt sich dadurch, welche Aspekte wirken, um den Raum aufzuspannen. Dabei gibt es eine Reihe von Aspekten, die dazu führen, dass der Kontingenzraum größer wird. Diese sind in der Abbildung dunkel dargestellt und wirken von innen gegen die Grenze des Kontingenzraums. Hell dargestellt sind hingegen die Kräfte, die zu einer Begrenzung des Raums führen. Beispielsweise wurden die Hochschulen mit Pandemieeintritt vor die Herausforderung gestellt, dass bekannte Rahmenbedingungen aufgelöst oder ausgesetzt wurden. Dies bewirkte zunächst, dass Hochschulen zur Ausgestaltung ihrer jeweiligen Situation mehr Möglichkeiten zur Verfügung hatten - der Kontingenzraum wurde größer. Dennoch befanden sich die Hochschulen nicht in einem Vakuum, zumal immer wieder neue rechtliche Verordnungen erlassen wurden. Diese gaben dem Kontingenzraum eine Limitation. So wirkten hier zwei Kräfte entgegengesetzt auf die Grenzen des Kontingenzraums. Setzt man diesen Gedankengang für die weiteren Aspekte fort, so wurde eine Bandbreite der Kontingenz aufgespannt, innerhalb derer sich Hochschulen bewegten.

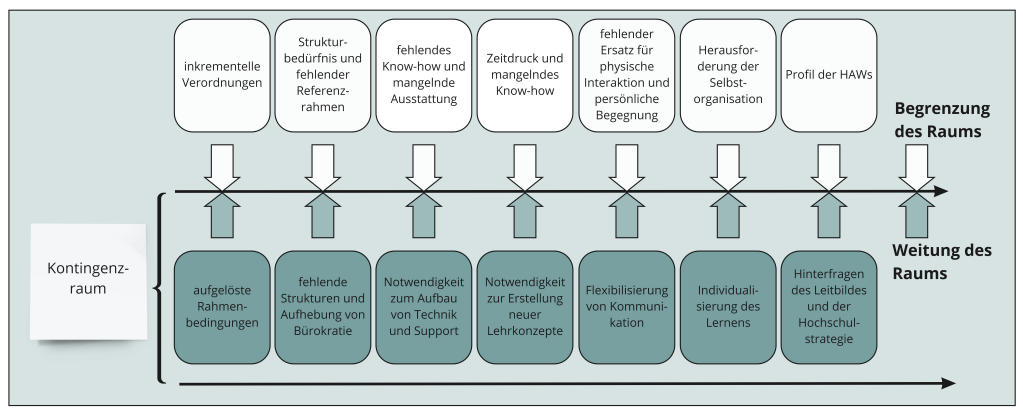

Abbildung 26: Übersicht Kontingenzbedingungen 
Bei der nun folgenden Zusammenführung und der Interpretation der Zugänge sollen die Ausgestaltung dieses Kontingenzraums sowie der Umgang mit den Kontingenzbedingungen und den Kontingenzgrenzen im Vordergrund stehen. Unter Rückgriff auf die übergeordnete Forschungsfrage (s. Kapitel 3), welche Entwicklungspfade post Corona für die Hochschulen aus den vergangenen digitalen Semestern abgeleitet werden können, wird hier betrachtet, welche Handlungserfahrungen die Hochschulen in den vergangenen Semestern gemacht haben. Rückblickend handelt es sich hierbei um einen inkrementellen Prozess $^{7}$ (s. Abbildung 27).

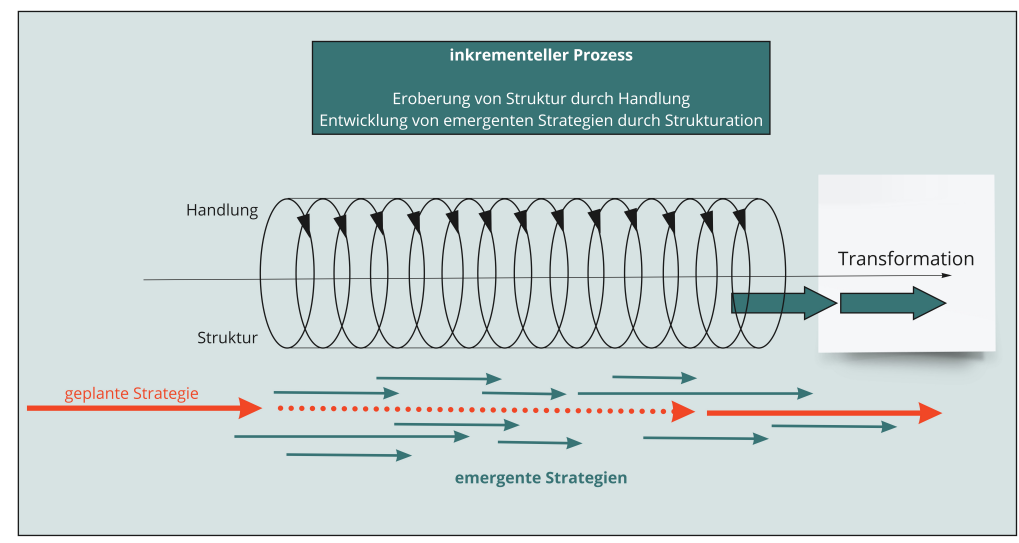

\section{Abbildung 27: Zusammenhang zwischen Handlung, Struktur und Strategie}

Die dadurch fehlende Struktur musste Stück für Stück durch Handlungen der Akteur*innen wiedergewonnen werden. Diese Dualität aus Handeln und Struktur geht auf Giddens zurück (Giddens, 1984) und wird als Strukturation bezeichnet. Der Zusammenhang ist in dem

7 Als inkrementellen Prozess werden in diesem Zusammenhang iterative Schritte verstanden, in denen aufgrund von Notwendigkeit Handlungen vollzogen werden und dadurch Struktur gewonnen wird. Diese Struktur wird im nächsten Schritt reflektiert. Es erfolgt ein neuer Handlungsschritt, der dazu führt, dass die Struktur überarbeitet, verworfen oder bestätigt wird. Dieser Ablauf kann sich beliebig oft wiederholen. 
Sinne rekursiv, als dass vorangegangene Strukturbildungen - also die Ergebnisse vorangegangener Handlungen - Restriktionen für das aktuelle Handeln bilden, aber auch Möglichkeiten dafür bereitstellen. In dieser rekursiven Bewegung von Struktur und Handlung ist einbezogen, dass es nichtintendierte Handlungsfolgen gibt und dass Strukturbildungen in Zeit und Raum weit über die Reichweite der Handlungen einzelner Akteur ${ }^{\star}$ innen hinausweisen. Gleichwohl werden Strukturen nur durch das Handeln von Akteur*innen wirksam. Diese setzen sie gewissermaßen in Gang und verleihen ihnen erst Realität.

Hochschulen hatten in der Corona-Krise einen Strukturationsprozess $\mathrm{zu}$ bewältigen, in welchem Handlung und Strukturbildung in sehr gedrängter Weise aus teilweise sich überstürzenden, aufeinander folgenden und neuen Strukturen - etwa digitalen Konferenzsystemen - neue Handlungsmuster generierten und umgekehrt aus den sich entwickelnden Handlungsmustern neue hochschulische Strukturen entstanden.

Infolge dieser inkrementellen Vorgehensweise rückten bereits vor der Corona-Pandemie geplante Strategien der Hochschule zunächst in den Hintergrund oder wurden sogar ganz ausgesetzt. Soweit dieser Strukturationsprozess reflektiert erfolgte und die Hochschulen sich nicht einfach treiben ließen, lässt sich daraus schließen, dass hochschulisches Handeln bestimmten Zweckrationalitäten folgte. Diese Zweckrationalitäten bildeten gewissermaßen einen Korridor, in dem sich dem Handelnden relevante Probleme stellten sowie Lösungen dafür entwickelt wurden und in dem die Akteur*innen wiederum diese Probleme und Lösungen deuten und bewerten mussten. Durch den Prozess der Strukturation traten daher zunächst emergente Strategien in den Vordergrund. In Bezug auf die übergeordnete Frage nach der Pfadentwicklung wird zu klären sein, wie emergente Strategien schließlich wieder durch geplante Strategien nicht ersetzt, aber zumindest ergänzt werden oder in solche übergehen und ob die Hochschulstrategie, die vor der Pandemie entwickelt wurde, eine Disruption erfährt oder in eine Pfadentwicklung mündet. Dieser nach vorne gerichtete Blick auf die Gestaltung des Möglichkeitsraums wird jeweils im Rahmen der Handlungsimpulse behandelt. 
Bis $\mathrm{zu}$ diesem Zeitpunkt haben sich drei wesentliche Dimensionen gezeigt, die für die Gestaltung der Hochschulen während der Pandemie von hoher Relevanz waren. Diese sind (1) die Akteur ${ }^{\star}$ innen, die (2) im Wechselspiel zwischen Handlung und Struktur einen Strukturationsprozess beschreiten und dadurch (3) emergente Strategien in Erscheinung treten lassen. Dieser Dreiklang von Akteur*innen, Strukturation und Strategie wird aufgegriffen und die zusammenführenden Ergebnisse der drei Zugänge werden entsprechend diesen Dimensionen geclustert. Abbildung 28 verdeutlicht anhand dessen den Aufbau der nun folgenden Unterkapitel.

Die Beschreibung der Leitmotive (in der Abbildung dunkel dargestellt) entspricht einer Verdichtung und Interpretation der Daten unter $\mathrm{Zu}$ sammenführung der Perspektiven der Hochschulleitungen, Lehrenden und Studierenden. Davon ausgehend werden in einem nächsten synthetisierenden Schritt Handlungsfelder (hier hell dargestellt) identifiziert, die für die zukünftige Pfadentwicklung an Hochschulen wesentlich sind. Im Bereich der Handlungsfelder werden Impulse gegeben, die jeweils wieder auf die Dimension der Akteur ${ }^{\star}$ innen, Strukturation und Strategie bezogen sind. Im Folgenden werden jeweils entlang einer Dimension (Akteur*innen, Strukturation, Strategie) zunächst die Leitmotive und direkt anschließend die Handlungsfelder diskutiert. 


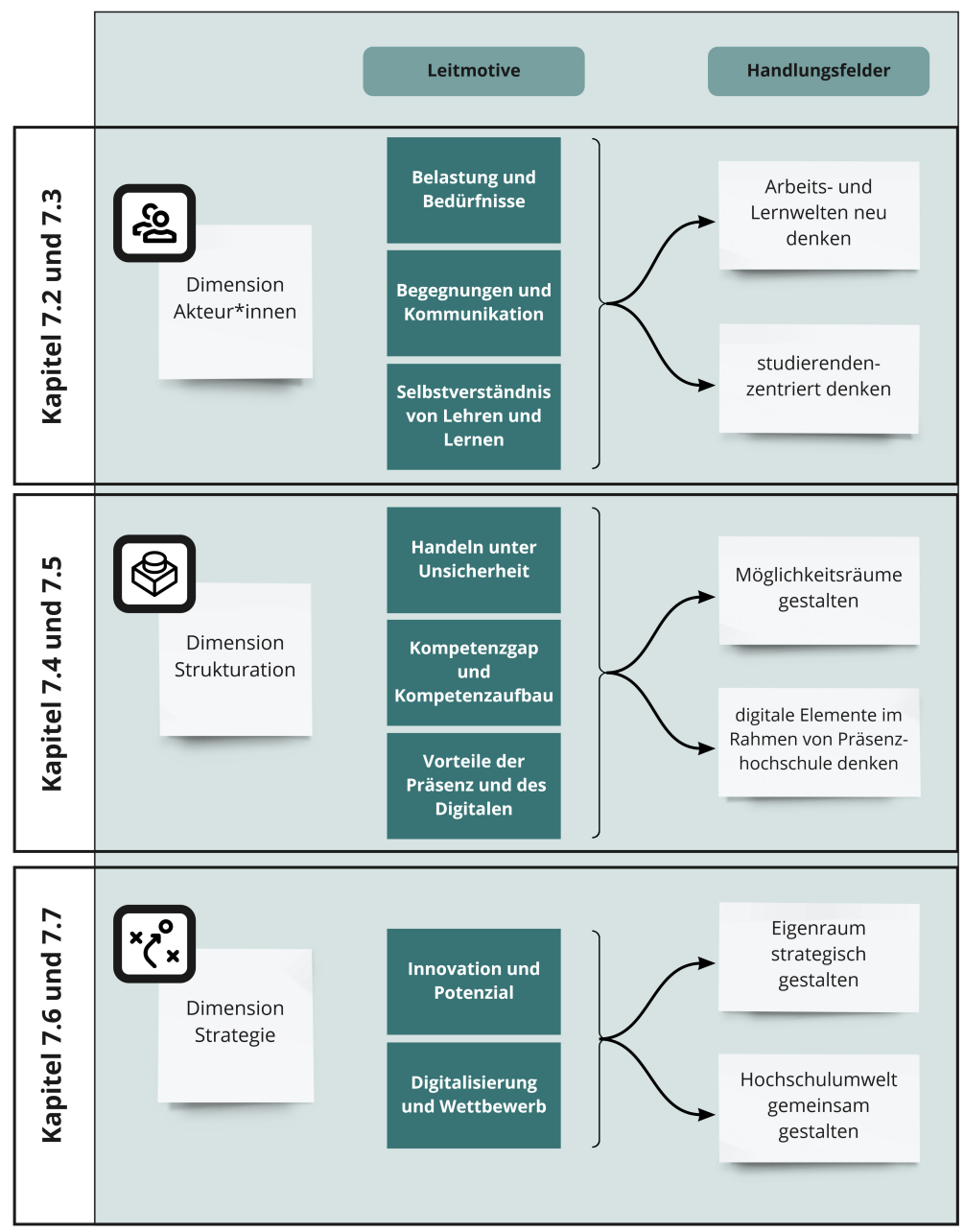

Abbildung 28: Übersicht über die Leitmotive und die Handlungsfelder 


\subsection{Leitmotive in der Dimension Akteur*innen}

In dieser Dimension stehen die handelnden Akteur*innen und ihre subjektspezifischen Perspektiven im Fokus. Auch wenn Akteur ${ }^{\star}$ innen und Strukturation nicht voneinander zu trennen sind, soll in diesem Abschnitt der Fokus stärker auf das subjektbezogene Verhalten im sozialen Kontext gelegt werden. Dies erfolgt über die Leitmotive Belastung und Bedürfnisse, Begegnungen und Kommunikation sowie das Selbstverständnis von Lehren und Lernen (s. Abbildung 29).

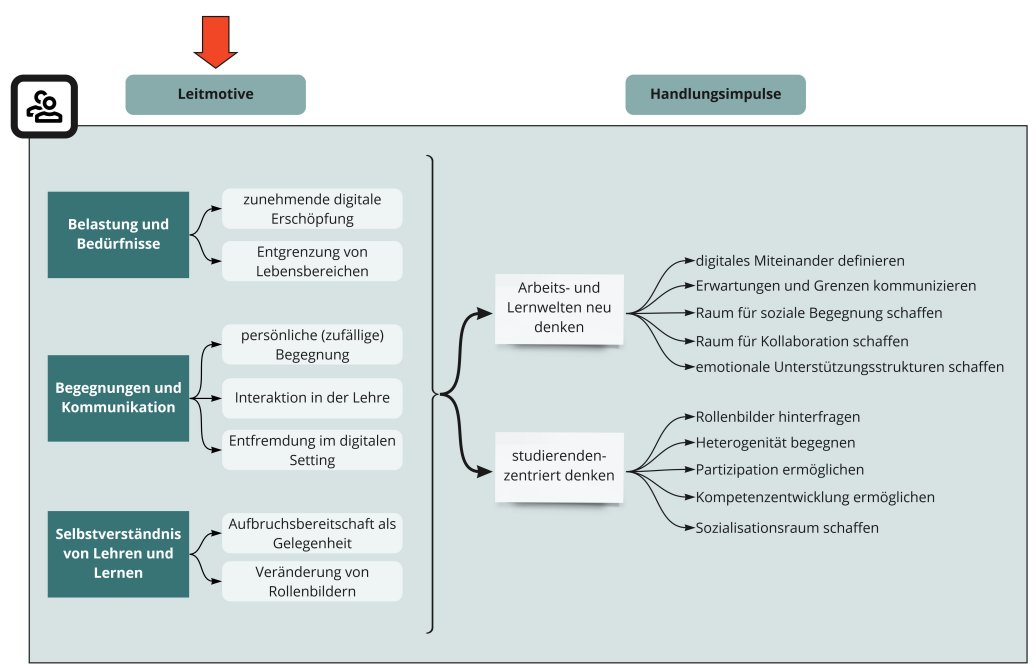

Abbildung 29: Leitmotive in der Dimension Akteur*innen

Die Hochschule tritt in der Dimension Akteur*innen verstärkt als sozialer Beziehungsraum in Erscheinung. In diesem - zuletzt rein digital umgesetzten - Raum verhalten sich einzelne Personen ihrer Individualität entsprechend und interagieren sehr unterschiedlich im sozialen Gefüge. Ausgehend von den Ergebnissen der Interviews (s. Kapitel 4, Kapitel 5 und Kapitel 6) sind drei wesentliche Aspekte wiederholend aufgetreten. Das erste Leitmotiv umfasst stärker die entstandenen Belastungen und Bedürfnisse (s. Kapitel 7.2.1). Der zweite wesentliche Aspekt bezieht sich auf den Bereich der Begegnung und 
Kommunikation (s. Kapitel 7.2.2). In Bezug auf die konkrete Lehr-/ Lernsituation wurde die Arbeit an einem neuen Selbstverständnis als relevant erfasst (s. Kapitel 7.2.3).

\subsubsection{Belastung und Bedürfnisse}

Zunehmende digitale Erschöpfung: Ein erster wesentlicher Fokus liegt hier auf dem wiederkehrenden Leitmotiv der digitalen Erschöpfung. Diese wurde vor allem auf der Ebene der Lehrenden und Studierenden geäußert. Beide Gruppen weisen auf eine hohe physische und psychische Belastung hin, die dadurch entsteht, dass sie Zeit vor dem Bildschirm verbringen und wenig bis gar keinen physischen Kontakt $\mathrm{zu}$ anderen haben. Sie zeigt sich in Überarbeitung, Erschöpfung, Ermüdung und Bewegungsmangel. Die geäußerte digitale Erschöpfung ist ein Zeichen dafür, dass die Lehr-/Lernmodelle in der Art, in der sie in den Corona-Semestern eingesetzt werden, nicht von dauerhaftem Bestand sein können. Sie zeigt auch die Notwendigkeit, dass Konzepte entwickelt werden müssen, die das Digitale in einem didaktisch sinnvollen und nicht überfordernden Umfang in die Lehre integrieren. Ein weiteres Indiz dafür ist, dass die digitale Erschöpfung nicht direkt, sondern (teilweise) nach einer ersten Euphorie darüber, was alles digital möglich ist, einsetzte.

Die Art und Weise der derzeitigen Digitalisierung der Lehre beschreibt einen Übergangszustand und kann daher nicht als zukünftiges Konzept der befragten HAWs dienen. Zu Anfang der Pandemie stand im Vordergrund, den Studienbetrieb aufrechtzuerhalten. An diesem Ziel orientiert entwickelten Akteur ${ }^{*}$ innen Handlungen, die zu einer neuen Strukturierung des Arbeits- und Lernalltags führten. Durch die dann gelebte Praxis traten zusätzlich zum Normalbetrieb Belastungen (wie der hohe kapazitive Aufwand der digitalen Lehre) oder grundlegende Bedürfnisse (wie die nach sozialem Kontakt) stärker zutage. Darauf aufbauend entstanden neue, emergente Handlungsstrategien, zum Beispiel der vermehrte Einsatz von interaktiven digitalen Werkzeugen, um diesen Bedürfnissen zu begegnen. Durch diese Iteration wurde eine neue Struktur erarbeitet bzw. die bis dahin geltende Struktur ergänzt. 
Entgrenzung von Lebensbereichen: Der zweite Aspekt fokussiert die Entgrenzung verschiedener Lebensbereiche und das dadurch entstandene Gefühl von Belastung. Im Hometeaching und Homestudying ist das Private kaum vom Beruflichen zu trennen, was hauptsächlich durch die pandemiebedingte Situation ausgelöst wird. Die in den Interviews beschriebene Entgrenzung zwischen dem privaten und dem professionellen Lebensbereich wurde von Studierenden und Lehrenden formuliert. Lehrende gaben zudem an, durch das parallele Homeschooling bzw. die zeitgleiche Kinderbetreuung stark gefordert zu sein. Diese Anforderungen können auf Studierenden in Elternschaft ebenfalls übertragen werden, auch wenn in der hier befragten Gruppe das Thema explizit nicht vorgebracht wurde. Hier zeigte sich der inkrementeller Prozess. Eine Handlungsweise wurde eingeübt und dadurch eine fehlende Struktur neu definiert. Auf der Seite der Studierenden steht die räumliche Begrenztheit stärker im Fokus und das daraus resultierende Fehlen eines gut nutzbaren Arbeitsplatzes, der sich von Privatleben und Schlafplatz abgrenzt. Die Studierenden berichteten ebenfalls über eine Verwischung der Grenze zwischen Privatem und Studium sowie über die dadurch entstandenen Konflikte. Die Lehrenden bewerteten dagegen ihre Situation als privilegiert.

Insgesamt ließen sich intra- und interindividuelle Rollenkonflikte bei den Akteur*innengruppen beobachten. Hochschulleitungen beispielsweise mussten einerseits ihrer Führungsrolle gerecht werden und wollten andererseits Erwartungen der Hochschulangehörigen erfüllen sowie Partizipation ermöglichen. Damit waren die Hochschulleitungen in ihrer Rolle und der damit ausgeübten Haltung in einem Konflikt zwischen verschiedenen Instanzen gebunden bzw. auf die aktive Mitarbeit der Kolleg*innen und deren Selbstorganisation angewiesen. Lehrende und Studierende beschreiben vielfach den individuellen Rollenkonflikt zwischen den Handlungsweisen in den unterschiedlichen Lebensbereichen. Auch wenn nach der Pandemie und mit der Aufhebung von Kontaktbeschränkungen einige dieser Probleme wegfallen, ist das Thema der neuen Definition von Rollenbildern vor allem in Hinsicht auf die Lehrendenrolle in neuen Lehrformatsettings zukunftsweisend und ein äußerst relevanter Bestandteil der zukünftigen Arbeits- und Lernwelt an Hochschulen. 


\subsubsection{Begegnungen und Kommunikation}

Persönliche (zufällige) Begegnung: Ein wesentlicher Aspekt hinsichtlich der Herausforderungen in den digitalen Semestern betrifft die fehlende (zufällige) physische Begegnung. Durch die Beschränkung der Kontakte und des Homestudyings bzw. Hometeachings fiel insbesondere eines weg: die persönlichen Begegnungen. Dieses Leitmotiv schien ein zentrales Element zu sein, das rückschlüssig auf viele weitere Kernthemen Einfluss hat. Das Sich-nicht-treffen-Dürfen war der Ausgangspunkt für alles Folgende. Es bedingte die Notwendigkeit der digitalen Lehre und damit auch die Frage nach dem Kompetenzgap (s. Kapitel 7.4.2), aber auch der digitalen Erschöpfung. Es sorgte dafür, dass das ganze Hochschulsystem, das auf diesen Begegnungen beruht, neu erfunden werden musste. Aber auch in dem Moment der Begegnung selbst lag ein tiefes Bedürfnis der Akteur*innen, das vielfach in den Interviews und Fokusgruppen zum Ausdruck gebracht wurde. Es scheint vor allem die zufällige, informelle Begegnung zu sein, die durch die digitalen Tools kaum aufgefangen werden kann.

Ähnliches berichteten die Studierenden in Bezug auf die Begegnungen in der Mensa oder zwischen den Veranstaltungen. Für diesen zufälligen Austausch ließ sich vor allem in der digitalen Umgebung kein funktionierendes Äquivalent finden, weil eben das Moment der Zufälligkeit eine wesentliche Rolle dabei spielte. Es gab zwar Bemühungen, informelle Treffen in digitale Strukturen zu überführen und damit eine Möglichkeit für einen Austausch zu schaffen, aber die zufällige Begegnung mit Hochschulangehörigen entfiel nahezu vollständig. Dieser Umstand wirkte insbesondere auf die individuell gefühlte Atmosphäre, die in keiner Weise digital so gestaltet werden konnte, dass sie einer tatsächlichen sozialen Interaktion entsprach. Es fehlte die soziale Einbettung, die für die Identifikation mit der Hochschule als Lebensraum notwendig ist. Insbesondere für Studierende in der Studieneingangsphase war es ohne persönliche Kontakte und Begegnungen im rein digitalen Semester nur sehr schwer möglich, sich kennenzulernen. Ebenso waren Lehrende weniger miteinander in Kontakt und teilweise seit Monaten nicht mehr an der Hochschule gewesen. Dies alles führte dazu, dass die Identifikation mit der eigenen Hochschule in den Hintergrund geriet. Es fehlten das Gemeinschaftsgefühl und 
das gegenseitige Begleiten durch Höhen und Tiefen, welches nur durch persönliche zwischenmenschliche Elemente erlebt werden kann. An dieser Stelle braucht es ein neues Verständnis und ein gemeinsames Commitment darüber, wie in Zukunft gemeinsam gearbeitet, gelehrt und gelernt werden wird.

Interaktion in der Lehre: Die zweite Herausforderung liegt in der Gestaltung von Interaktion in der Lehre. Vor allem im konkreten Lehr-/ Lernkontext ist die digitale Kommunikation zunächst eine behelfsmäßige. Die Lehrenden berichten davon, dass das direkte Feedback der Studierenden in der synchronen Veranstaltung nur schwer einzuholen ist. Das hat zum Grund, dass im digitalen Raum Gestik und Mimik zum großen Teil verloren gehen. Vor allem scheint das Thema der Anonymität eine zentrale Rolle zu spielen, wenn die Studierenden die Kameras ausgeschaltet haben.

Entfremdung im digitalen Setting: Die Anonymität und damit verbundene Entfremdung wird im direkten Zusammenhang mit der Kameranutzung diskutiert. Die aufseiten der Studierenden und Lehrenden als hinderlich empfundene ausgeschaltete Kamera zusammen mit dem Umstand, dass es scheinbar bisher kaum gelungen ist, daran etwas zu ändern, lässt die Vermutung zu, dass eine Etikette des Miteinanders im digitalen Raum fehlt. Fraglich bleibt allerdings, ob das Problem nur aufgrund der fehlenden Etikette besteht. Grundsätzlich scheint es ein kollektives Phänomen zu sein, da berichtet wurde, dass die Kameras nach und nach ausgeschaltet wurden, sobald dies ein ${ }^{\star} e$ Teilnehmende* $r$ der Gruppe machte. Das Thema der Etikette betrifft wiederum nicht nur die Lehre, sondern auch die Vorgänge in der Verwaltung oder in den Gremien der Hochschule. Im analogen Format existieren seit Langem kultivierte Verhaltensweisen der respektvollen Begegnung in Besprechungen. Auf allen Seiten gibt es Erwartungen, wie in den Austausch getreten wird, welches Verhalten angemessen ist und welche Kommunikationsregeln eingehalten werden sollten. Darüber herrscht zumindest grundsätzliche Einigkeit. In der digitalen Situation gibt es diesbezüglich zwar viele Vorschläge, aber diese konnten sich noch nicht insoweit festigen, dass sie als gesetzt gelten können. Das hat auch mit der grundsätzlich geringeren Verbindlichkeit im digitalen Bereich zu tun. Wenn in einer physischen Besprechung nebenher E-Mails beantwortet werden, fällt das Tippen auf. In einem 
digitalen Meeting ist dies bei ausgeschalteter Kamera nicht der Fall. Bei ausgeschaltetem Mikrofon und ausgeschalteter Kamera herrscht Anonymität. Diese erschwert das digitale Lehren und Lernen in synchronen Veranstaltungen massiv. Zudem verändert sich die Diskussionskultur. Diskussionen verlaufen im digitalen Kontext seriell. Zur Sprache kommt, wer sich gemeldet hat, und zwar in der Reihenfolge der Meldungen. Eine Gruppendiskussion scheint kaum möglich, da die digitale Situation viel stärker moderiert und formalisiert werden muss als die vergleichbare Situation in Präsenz.

\subsubsection{Selbstverständnis von Lehren und Lernen}

Aufbruchsbereitschaft als Gelegenheit: In der Dimension Akteurinnen wurde als weiteres Leitmotiv das Selbstverständnis von Lehren und Lernen in den Blick gerückt. Durch die derzeitige Situation, die durch Aufbruchsbereitschaft geprägt ist, ist die Gelegenheit für eine Diskussion über dieses Selbstverständnis günstig. Wie soll gemeinsam gelernt und gelehrt werden? Diese grundsätzliche Frage ist nicht neu. Selten jedoch war die Gelegenheit so günstig, darüber zu diskutieren, wie Lehren und Lernen zukünftig gestaltet sein sollen. Denn in einem funktionierenden und in geordneten Bahnen sich bewegenden System ist die Aktivierungsenergie, die benötigt wird, um Veränderungen herbeizuführen, ungleich höher als in der gegenwärtigen, grundsätzlich offenen und von Aufbruchsbereitschaft gekennzeichneten Situation. Der Prozess des Diskutierens über die zukünftige Gestaltung von Lehre und Lernen kann nur inkrementell mit allen hochschulischen Akteur*innen gemeinsam stattfinden. Im Rahmen der Pandemie haben zeigten sich viele Ausprägungen digitaler Lehre. Durch unterschiedlichste Handlungen erarbeiteten sich Akteur*innen neue Lehr-/ Lernstrukturen. Von diesem Prozess ausgehend muss die Frage gestellt werden, an welchen Maßstäben sich zukünftiges Lehren und Lernen orientiert. Im Kontext welchen Referenzrahmens soll die Entwicklung zukünftiger Lehr-/Lernsettings also gedacht werden?

Ein Teil dieses Selbstverständnisses von Lehren und Lernen liegt in bereits diskutierten Rahmenbedingungen wie der sich verändernden Kommunikationskultur. Es ist nun geboten, die Schwierigkeiten zu 
überwinden und die jeweiligen Stärken iterativ herauszuarbeiten. Was anfangs der Pandemie noch vielfach als Durchhalten abgetan wurde immer mit dem Blick, dass danach alles wieder wie vor Corona wird - ist inzwischen schon selbst im Prozess. Dabei stellt sich die Frage: im Prozess wohin? Doch längst ist die Digitalisierung der Lehre nicht mehr nur notwendiges Übel, sondern überzeugt an manchen Stellen auch durch neue Optionen.

Das Hineingeworfen-Sein, das notwendigerweise reaktives Verhalten evoziert, weicht dem Wunsch, die Digitalisierung als Chance zur Veränderung zu begreifen und den Digitalisierungsschub zu nutzen, um Innovation zu entwickeln.

Veränderung von Rollenbildern: Zuletzt sind es auch die Rollenbilder, die neu überdacht werden müssen. Dafür muss die zugrunde liegende Frage nach dem Selbstverständnis von Lehren und Lernen an Hochschulen neu beantwortet werden. Das betrifft auch die Rollen der Lehrenden selbst und die Art, wie diese ihr Handeln in ihrer Rolle begreifen wollen. Lehrende werden zukünftig noch stärker gefordert sein, mit ihren Studierenden auf Augenhöhe zu kommunizieren, sie als ein Gegenüber verstehen, das nicht entsprechend einem Gefäß gefüllt werden will, sondern das sich in einem iterativen und begleiteten Prozess selbst Kompetenzen aneignen möchte. Die Lehrenden sind immer mehr gefragt, eine entsprechende Atmosphäre und Lernumgebung zu schaffen, damit Studierende sich selbst mit den nötigen Future Skills ausstatten können. In der Lehrendenrolle geht es vermehrt um die Ansprache des Einzelnen. Wissen wird dann entsprechend nicht konsumiert, sondern sich selbst aktiv angeeignet. Dies kann am besten in kleinen Gruppen und interaktiv im kommunikativen Austausch geschehen, so die Befragten.

\subsection{Handlungsimpulse in der Dimension Akteur*innen}

In der Dimension der Akteur*innen wurden in Kapitel 7.1 drei verschiedene Leitmotive aus der Zusammenführung der Ergebnisse und der Interpretation der Interviews und Fokusgruppen vorgestellt. Diese adressieren Bedarfe hinsichtlich des Umgangs mit Belastungen und Bedürfnissen, der Begegnungen und der Gestaltung der Arbeits- und 
Lebensbereiche sowie die Frage zur zukünftigen Lehr-/Lernkultur. Abgeleitet von den genannten Leitmotiven wurden zwei Handlungsfelder identifiziert (s. Abbildung 30), denen jeweils weiterführende Impulse zugeordnet sind.

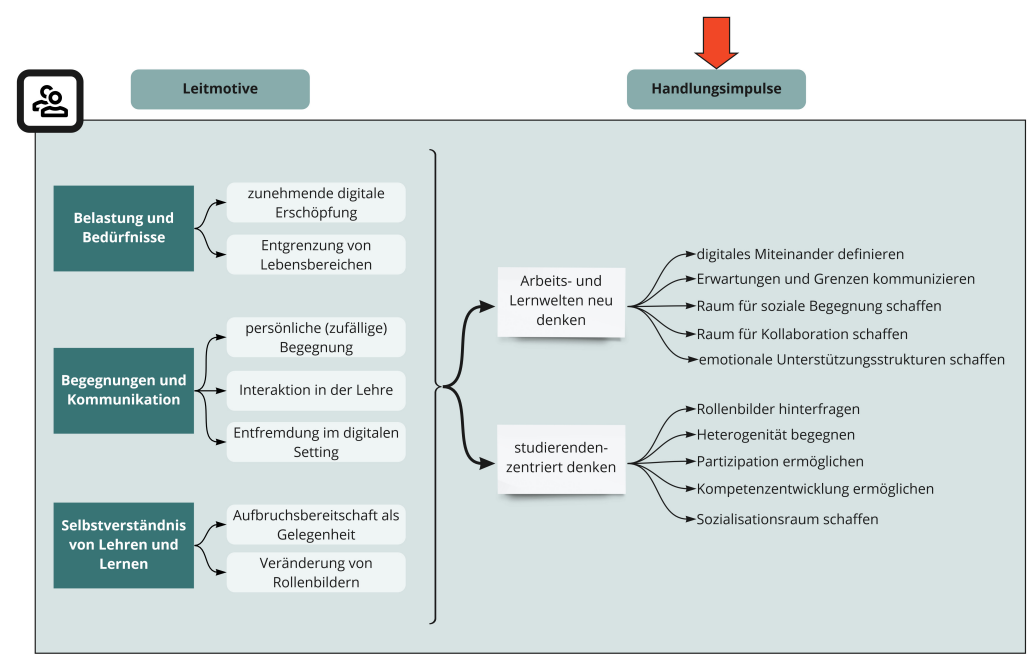

Abbildung 30: Handlungsimpulse in der Dimension Akteur*innen

\subsubsection{Arbeits- und Lernwelten neu denken}

Viele Bereiche, die mit der Art und Weise des Zusammenarbeitens zu tun haben, tangieren auch die Frage danach, wie die zukünftigen Arbeits- und Lernwelten aussehen. Hier gibt es einige starke Zusammenhänge zur Dimension der Strukturation, aber auch einen entscheidenden Unterschied: Im Rahmen dieser Betrachtung soll genauer untersucht werden, welche zwischenmenschlichen und sozialen Aspekte eine Rolle spielen. Die hinter diesem Handlungsfeld liegenden Leitmotive beziehen sich einerseits auf die Formulierung von Belastung und Bedürfnissen und andererseits thematisieren sie genauer den Kontext der Begegnung und Kommunikation. In eben diesen Leitmotiven liegt viel Substanz, die auf die Ausgestaltung des gemeinsamen Miteinan- 
ders abzielt und die Hochschule als Lebenswelt begreift, in der Individuen Entwicklung erfahren.

Grundsätzlich sind viele dieser Themen vor allem in der Corona-Situation relevant. Die durchgehende digitale Erschöpfung, die soziale Isolation oder die Doppelbelastung durch den Wegfall der Kinderbetreuung wird sich vermutlich post Corona relativieren. Am Verhalten der Befragten während dieser Extremsituation lässt sich viel darüber lernen, was den Akteur*innen an HAWs grundsätzlich an der gemeinsamen Gestaltung der Arbeits- und Lernwelt Hochschule wichtig ist. Viele Aspekte sind relevant für eine zukünftige Ausrichtung der Hochschulen und können Hinweise für die Orientierung im Kontingenzraum geben.

Digitales Miteinander definieren: Ein erster Impuls ist folgerichtig die notwendige Definition des digitalen Miteinanders. Wie im analogen Setting im Seminar- oder Sitzungsraum miteinander umgegangen wird, ist (weitestgehend) geklärt. Dem Miteinander im digitalen Raum fehlt dieser über Jahrzehnte etablierte Referenzrahmen für Verhalten. Gemeinsam müssen (ggf. aufbauend auf bereits vorhandenen Vorschlägen für eine digitale Etikette) Vereinbarungen getroffen werden, damit die digitalen Situationen auch zukünftig gewinnbringend sind. Durch die Notwendigkeit der schnellen Digitalisierung, die auch die Kommunikation betraf, gab es keinen Entwicklungszeitraum für eine aus der Gewohnheit gewachsene Etikette. Vielmehr muss Verhalten im digitalen Raum explizit zum Thema gemacht werden. Das betrifft nicht nur Verhalten im Kommunikationskontext, sondern meint auch eine digitale Verhaltenskultur allgemein. Aspekte dieser Verhandlung sind beispielsweise der Umgang mit der Kamera, die digitale Verbindlichkeit, Diskussionsfreiheit, der Umgang mit dem Chat und Ähnliches. Dazu muss ein Prozess ins Leben gerufen werden, der alle Akteur*innen gleichermaßen einbezieht. Nur gemeinsam kann der kommunikative Raum Hochschule gestaltet werden.

Erwartungen und Grenzen kommunizieren: Ebenso gehört zum Umgang im digitalen Setting die klare Kommunikation von Erwartungen und Grenzen. Im Prozess der Gestaltung der digitalen Kultur spielt die Entgrenzung zwischen verschiedenen Lebensbereichen eine zentrale Rolle. In der Pandemiesituation fand diese Entgrenzung au- 
tomatisch durch die räumliche Restriktion statt. Durch Homeoffice, Homestudying und Hometeaching waren und sind die allermeisten Hochschulangehörigen dazu genötigt, zu Hause einen Lern- und Arbeitsplatz zu etablieren. Unabhängig von der privaten Situation und privaten Pflichten ließ sich über alle Befragte hinweg beobachten, dass sich die Grenzen zwischen Studium/Beruf und Privatem verwischten. Post Corona werden wieder mehr Möglichkeiten bestehen, diese Grenzen zu gestalten. Dennoch wird, sollte der Digitalisierungsschub genutzt werden und weiterhin digitales Lernen und Arbeiten möglich sein, eine Situation wie vor Corona mit (zumindest in den meisten Fällen) klarer Abgrenzung zwischen professionellem Kontext und privatem Lebensbereich nicht mehr möglich sein. Es wird also dabei um die Frage gehen, wie diese Grenze nach Corona gestaltet wird. Es ist $\mathrm{zu}$ bedenken, dass alle beteiligten Gruppen hier für sich selbst reflektieren, wo die Grenze verlaufen kann und wie restriktiv diese sein muss. Eine wesentliche Notwendigkeit an dieser Stelle ist Transparenz. Alle sind dazu aufgefordert, ihre Erwartungen an die Akteur*innen zu kommunizieren, mit denen sie zusammen lernen und arbeiten. Es ist aber auch geboten, die eigenen Grenzen klar zu kommunizieren. $\mathrm{Ob}$ Hochschulangehörige jederzeit digital erreichbar bleiben wollen, welches Medium bei der Kontaktaufnahme genutzt werden soll oder ob es klare Arbeits- bzw. Privatzeiten gibt, liegt einerseits am Tätigkeitsprofil, wird andererseits zukünftig in flexiblen Arbeitsräumen vermutlich aber immer mehr in die Hände der einzelnen Person gegeben. Bei sich entwickelnden, vielfältigen Arbeits- und Lernorganisationen ist daher die gemeinsame Kommunikation ein zentraler Bestandteil eines gelingenden Miteinanders.

Raum für soziale Begegnung schaffen: Neben der Gestaltung des digitalen Raums muss vor allem der physische Raum für soziale Begegnungen geschaffen werden. Zusätzlich zu den gerade beschriebenen, strukturellen Kommunikations- und Organisationsprozessen ist ein wiederkehrendes Motiv der fehlende informelle Austausch. In der Situation nach der Corona-Pandemie werden die Studierenden wieder (mehr) an die Hochschulen kommen. Die Corona-Situation hat gezeigt, dass die soziale und persönliche Begegnung ein wesentliches Merkmal von HAWs ist. Hochschulen zeichnen sich nicht nur dadurch aus, gemeinsam einen Fachinhalt zu bearbeiten, sondern es 
geht darüber hinaus, was als Studierendenleben oder Campus-Feeling tituliert wurde. Dieser soziale Austausch hat während der Pandemie deutlich gefehlt. Es scheint kein funktionales Äquivalent im digitalen Raum zu geben, welches die soziale Interaktion in dem Maße ersetzen kann, wie es in der direkten Begegnung geschieht. Die digitale Welt kann die soziale Eingebundenheit nicht ersetzen. Wenn Hochschulen in Zukunft neugestaltet werden, ist es erforderlich, die Arbeits- und Lernwelt nicht nur prozesshaft und hinsichtlich ihrer grundsätzlichen Funktionsfähigkeit zu denken, sondern das zentrale Moment des Lebensraums Hochschule zu gestalten. Dies darf nicht nur theoretisch geschehen, sondern muss sich durch die konkrete Gestaltung von Räumen für Begegnung zeigen. Maßnahmen der Campusgestaltung sind kein neues Anliegen der Hochschulen. Doch in der Pandemie zeigte sich, wie (hoch)relevant das Thema Hochschule als Raum der Begegnung für die Studierendenzufriedenheit ist. Es stiftet Identifikationsmöglichkeit über den Studieninhalt hinaus. Die Gestaltung des Campus ist zwar kein vernachlässigtes, aber möglicherweise unterschätztes Kriterium bei der Wahl des Studienorts. Hier liegt eine Aufgabe bei der Campusentwicklung, die Erkenntnisse aus der Corona-Pandemie aufzunehmen und in sinnvolle Konzepte und möglichst auch in bauliche Maßnahmen umzusetzen, um den Raum Hochschule für alle wertschöpfend $\mathrm{zu}$ gestalten und die soziale Begegnung in den Vordergrund zu rücken. Es ist Aufgabe der Bildungspolitik, diesen Bedarf für Hochschulen im Blick zu haben und Hochschulen die Möglichkeit zu geben, sich entsprechend auszustatten.

Raum für Kollaboration schaffen: Die Lehre betreffend stellt sich anschließend an die Gestaltung des Campus die Frage, wie Lehr- und Lernräume zukünftig ausgestattet sein müssen, um Raum für Kollaboration zu schaffen. Im Pandemiegeschehen selbst postulierten Stimmen im öffentlichen Raum immer wieder, die Hochschulen hätten zukünftig weniger Raumbedarf. Dennoch wird womöglich der Raumbedarf nach der Pandemie höher sein, wenn Lehr-Lernkonzepte entsprechend weiterentwickelt werden. Das liegt hauptsächlich daran, dass die Arten des Miteinanderarbeitens in Frage gestellt bzw. neu konzipiert werden müssen. In den Interviews und Fokusgruppen zeichnete sich eine Argumentationslinie ab, die in der Präsenzlehre weg von Großveranstaltungen hin zu kleinen, kollaborativen und interaktiven 
Veranstaltungen führte. Große Hörsäle werden in Zukunft möglicherweise seltener gebraucht werden. Dafür gibt es an Hochschulen nur wenige Räume, die für Kleinstgruppen genutzt werden können. Digitalisierung ist dabei nicht nur Voraussetzung für die Umsetzung von mediendidaktischen Lehr-/Lernformaten, sondern sie ist ein Aushängeschild.

Emotionale Unterstützungsstrukturen schaffen: Neben den räumlichen Gegebenheiten ist die emotionale Unterstützung der Hochschulangehörigen, vor allem der Studierenden, wesentlich. Oft waren Unterstützungsangebote nötig, um sich im neuen Arbeits- und Lernalltag in der pandemiebedingten Situation zurechtzufinden. Es wurden Strukturen nicht nur experimentell gewonnen, sondern auch gezielt neue Supportstrukturen geschaffen. Einen großen Bedarf scheint es nach wie vor hinsichtlich struktureller emotionaler Unterstützung zu geben. Dies ist ein Bereich, der auch nicht einfach durch die Einrichtung eines Schulungsangebots abzubilden ist. Emotionale Unterstützung erfahren Menschen in Krisensituationen durch die verschiedensten Kontakte. Auch wenn es an Hochschulen Angebote der Seelsorge oder der psychosozialen Beratung gibt, ist es wichtig, Hochschulangehörige und vor allem Funktionsträger ${ }^{\star}$ innen für die emotionale Unterstützung der Studierenden zu sensibilisieren. Wenn hier keine professionalisierte psychosoziale Beratung durch Hochschulleitungen und Lehrende stattfinden kann, müssen die Akteur*innen, die im Erstkontakt mit Hilfesuchenden stehen, entsprechend qualifiziert reagieren können und die Unterstützungsangebote der Hochschule kennen, um weitere Maßnahmen vorschlagen und mögliche Lösungswege aufzeigen $\mathrm{zu}$ können. Ein hohes Maß an Kommunikation und Transparenz ist nötig, damit Informationen bezüglich Beratungsmöglichkeiten fließen können.

\subsubsection{Studierendenzentriert denken}

Studierendenzentriert zu denken hat zwei ganz wesentliche Momente: das der Partizipation der Studierenden und die Frage, wofür Lehre und damit letztlich Hochschule gebraucht werden. Im Leitmotiv des Selbstverständnisses von Lehren und Lernen zeigte sich, dass analy- 
siert werden muss, welche Aspekte des Lehrens und Lernens nach der Pandemie zukunftsweisend sind und von welchem Referenzpunkt ausgehend die zukünftige Lehr-/Lernkultur entwickelt werden soll. Ein Aspekt dabei ist, die Studierenden in den Mittelpunkt der Betrachtung zu rücken. Damit ist gemeint, dass Hochschule aus der Perspektive der Studierenden gedacht und gestaltet werden kann. Wesentlich ist dabei auch, welches Verständnis Hochschulleitungen und Lehrende von den Studierenden und deren Leben haben. Der Fokus sollte auf Studierende als Individuen auf einem jeweils zu gestaltenden Bildungsweg liegen und nicht auf der Gruppe von Studierenden als Produkt der Hochschullaufbahn bzw. als Kund*innen von Lehre. Ist das Studierendenbild in dieser Weise gedacht, ist es möglich, die Gruppe der Studierenden in die Gestaltungsprozesse der Hochschule nach der Corona-Pandemie mit einzubeziehen.

So kann die Studierendenperspektive wertvolle Impulse für die Gestaltung des Kontingenzraums spielen. Durch sie sind, wie in den Leitmotiven gezeigt, im Rahmen der Kontingenz emergente Strategien in Erscheinung getreten und damit Strukturationsprozesse betrieben worden. Darauf aufbauend wurden folgende Aspekte herausgearbeitet, die den Handlungsimpuls der Studierendenzentrierung stützen.

Rollenbilder hinterfragen: Anknüpfend an den oben ausgeführten Gedanken ist es eine wesentliche Notwendigkeit, Rollenbilder zu hinterfragen - und zwar auf der Seite der Lehrenden und der Studierenden. Studierende müssen mehr in die Position versetzt werden, ihre Bildungsbiografie aktiv zu gestalten. Diese Möglichkeit muss ihnen eröffnet werden, sie müssen sich aber auch aktiv für diese Rolle entscheiden. So können die Studierenden die Flexibilität innerhalb ihres Studiums stärker nutzen, aber auch zu Gesprächspartner*innen bei der Frage nach der Pfadentwicklung von Hochschule post Corona werden. Lehrende müssen sich dabei immer mehr als Lernbegleiter*innen verstehen. Sie können die Rolle des Coachs übernehmen und ihre Studierenden auf dem jeweiligen Bildungsweg unterstützen. Die durch die Digitalisierung hervorgerufene Flexibilisierung in Hinsicht auf Raum und Zeit zeigten, dass Studierende zukünftig ein höheres $\mathrm{Ma}$ an Eigenverantwortung für ihren Lernprozess übernehmen und Lehrende viel mehr im direkten Kontakt mit den Studierenden das Lernen begleiten müssen. Der Gedanke der sich hierbei verändernden 
Rollenbilder kann ein wesentlicher Aspekt bei der Entwicklung des Zukunftskonzepts der Lehre sein.

Heterogenität begegnen: Dabei sollte die ganze Bandbreite der Heterogenität der Studierenden mitgedacht werden. Dass es nicht „den“ oder „die“ Studierende ${ }^{\star} n$ gibt, ist kein neuer Gedanke, denn Förderprogramme auf Landes- und Bundesebene beziehen sich ausdrücklich auf die Heterogenität der Studierenden. Dabei wird sich vor allem auf Heterogenität in (sozialer) Herkunft, religiöser Weltanschauung, individueller Persönlichkeitsmerkmale oder der fachlichen Voraussetzungen in der Studieneingangsphase fokussiert. Das Pandemiegeschehen zeigte jedoch erneut, dass die Berücksichtigung von Heterogenität nicht vernachlässigt werden darf. Aus den Daten wurde ersichtlich, dass es unterschiedliche Umgangsweisen mit der digitalen Situation seitens der Studierenden gibt. Obwohl sie aus der Generation der Digital Natives stammen, ist es nicht möglich, Medienkompetenz im professionellen Lernsetting vorauszusetzen. Hier gab es in den Interviews und Fokusgruppen sehr unterschiedliche Beobachtungen, wie Studierende mit den digitalen Lernmedien zurechtkamen. Diese Heterogenität der Medienkompetenz war ebenso eine Herausforderung für die Lehre wie die Heterogenität in der Meisterung beispielsweise mathematischer Grundlagen. Grundtechniken am PC zu beherrschen, hat vor der Corona-Pandemie im Studium bereits eine Rolle gespielt. Nur war ein Mangel an solchen Kompetenzen für den Studienerfolg nicht so essenziell wie im Zeitraum der Pandemie. Es ist daher unbedingt notwendig, Studierenden die Möglichkeit zu geben, ihre Medienkompetenz weiterzuentwickeln. Corona brachte in allen Bereichen Gewinner und Verlierer hervor, so auch unter den Studierenden. Während einige eine hohe Medienaffinität mitbrachten und davon profitierten, gerieten medienferne Studierende eher in den Hintergrund. So ist es auch in der Präsenzlehre: Hier gibt es die wortstarken Studierenden und im anderen Extrem des Kontinuums diejenigen, denen das Sprechen vor der Gruppe schwerfällt. Dass die Vielfalt an didaktischen Möglichkeiten durch den Einbezug von digitalen Elementen an Präsenzhochschulen größer wurde, sollte dazu führen, dass differenzierter auf verschiedene Lern- und Persönlichkeitstypen eingegangen werden kann. Die Digitalisierung in der Lehre bietet zudem die Chance, aufbauend auf einem Methodenmix Lerngelegenheiten zu schaffen, die 
individualisierter auf die/den einzelne ${ }^{*} n$ Studierende ${ }^{\star} n$ eingehen. Das betrifft den Aspekt der Affinität zu verschiedenen Lernmaterialien. So kann mit einem Methodenmix das Lernen sehr abwechslungsreich gestaltet werden. Die Individualisierung der Lehrmethoden geht aber darüber hinaus und kann bei der jeweiligen Lehrveranstaltung zu einer detaillierten Binnendifferenzierung des Lerngegenstands werden. So kann eine Individualisierung nicht nur die Formate des Lehr-/Lernmaterials, sondern auch dessen Inhalt betreffen. Die Vorkenntnisse der Studierendengruppe sollte dabei genauer in den Blick genommen werden. Asynchrones Lernmaterial kann dann so erstellt und zur Verfügung gestellt werden, dass es dem jeweiligen Lernstand der Studierenden entspricht.

Partizipation ermöglichen: Das erhöhte Maß an Eigenverantwortlichkeit der Studierenden in den Corona-Semestern kann genutzt werden, um Partizipation in Zukunft zu ermöglichen. Auch wenn durch die Gremienstruktur und die Organisation auf Studierendenebene (z.B. Verfasste Studierendenschaft) eine Partizipation der Studierenden generell ermöglicht wird, ist der Wunsch nach mehr Partizipation gerechtfertigt, da diese (oft) an Hochschulen in der Hierarchiekette an unterster Stelle stehen und kaum Einfluss auf höhere Organisationsebenen haben. Bei Lehren und Lernen post Corona geht es aber ganz wesentlich um einen entscheidenden Lebensbereich und -abschnitt der Studierenden. Den Studierenden eine Stimme in diesem Gestaltungsprozess zu geben, die nicht nur pro forma abgebildet, sondern in der Realität gelebt wird, ist daher hoch relevant. Auf Studierendenseite ist es wichtig, diese Stimme zu nutzen, um konstruktiv mitzugestalten, Teilhabe einzufordern und sich Gehör zu verschaffen, auch im Kontext des Gestaltens der Hochschule. Schließlich sollten sich Akteur*innen in Entscheidungsfunktionen der Hochschulen im Klaren darüber sein, dass sie von den Studierenden ein ganz wesentliches Feedback zur Lehr-/Lernsituation während der Pandemie erhalten und damit Hochschule für zukünftige Studierende entsprechend gestalten können. Im Rahmen dieser Untersuchung wurde von Studierenden mehrfach positiv herausgestellt, dass sie sich durch die Fokusgruppen gehört fühlten. Hier konnten wichtige Implikationen dafür gewonnen werden, welche Bemühungen der Hochschulen aus der planerischen Ebene bei den Studierenden ankommen. Studierende haben ihre eigene Perspektive 
auf die Lehre, und diese ist wichtig, wenn die Gestaltungsfrage offen und in die Zukunft gerichtet gestellt werden soll. Es ist daher lohnenswert, Studierende in Entscheidungsprozesse stärker einzubinden.

Kompetenzentwicklung ermöglichen: Die Frage nach dem Profil und den Kompetenzen der Studierenden nach Studienabschluss rückt erneut in den Vordergrund. Dies zeigt sich vor allem dadurch, dass sich durch die Pandemie nicht nur die Hochschullandschaft erheblich verändert, sondern auch Berufsbilder und Arbeitsfelder neu entstehen bzw. modifiziert werden. Die Hochschule ist dabei gefragt, die neuen Berufsbilder zu evaluieren und ihre Studiengänge entsprechend anzupassen, wenn sie Studierende qualifizieren will, handlungskompetent in ihrem zukünftigen Beruf agieren zu können. Dabei muss in den Blick rücken, was die Absolventinnen zukünftig brauchen. Vor allem hinsichtlich neuer Technologien, Medien, Kommunikationstechnologien und weiterer Innovationsfelder, wie KI oder VR/AR, ist es wichtig, dass Studierende die Möglichkeit haben, entsprechende Kompetenzen in ihrem Studium zu erlangen. Die Hochschulen können dies auf unterschiedlichen Wegen leisten. Zum einen sollten sie durch die Schaffung einer entsprechenden Kultur dafür sorgen, dass Studierende sicher im digitalen Raum agieren und mit informations- sowie medientechnischen Grundausstattungen sicher umgehen können. Zum anderen sollte die Reflexion des Umgangs mit Technik und digitalen Medien stärker an den Hochschulen in den Fokus gestellt werden. Nötig hierzu ist eine entsprechende infrastrukturelle Ausstattung. Ebenfalls ist es hier wichtig, dass einzelne Hochschulen nicht abgehängt werden, da durch die Corona-Pandemie diese Themen von der Nische in die gesamte Breite der Hochschulen gerückt wurden.

Sozialisationsraum schaffen: Neben dem Thema der Kompetenzentwicklung steht für die Studierenden die Hochschule als Sozialisationsraum an wesentlicher Stelle. Hier entsteht ein starker Anknüpfungspunkt an die bereits beschriebenen Arbeits- und Lernwelten, der dennoch unter dem Aspekt der Studierendenzentrierung gedacht werden muss. Studierende erleben an der Hochschule während des Studierendenlebens einen wichtigen Abschnitt ihrer Sozialisation. An der Hochschule erfahren sie eine persönliche Prägung, die eng damit verknüpft ist, mit welchen Personen sie in Kontakt kommen. Nicht selten entstehen im Studium langfristige Freundschaften durch das ge- 
meinsame Gestalten des studentischen Lebens außerhalb der Lehrveranstaltung, aber auch durch gemeinsames Freud und Leid im Studium. Hochschulen bieten die Möglichkeit der Entwicklung, und zwar nicht nur der fachlichen, sondern auch der Persönlichkeitsentwicklung. Auf der Basis dieser Studie ist es ein zentraler Impuls, diesen Raum zur Sozialisation als physischen Campus unbedingt zu erhalten, um als HAW nicht ein wesentliches identitätsbildendes Merkmal zu verlieren. Hier gibt es eine besondere Herausforderung für die Hochschulen. Mit Blick in die Zukunft und auf die Frage danach, wie das Digitale und die Präsenz in einem Studium aufgeteilt werden sollte, ist hier ein Handlungsimpuls, die Studierenden in der Studieneingangsphase in die Hochschule zu holen. Vor allem in den ersten beiden Semestern sollte ausreichend Präsenzlehre stattfinden; insbesondere die Ersti-Wochen/Studieneingangswochen/Studienstartwochen sollten in Präsenz angeboten werden, denn der pandemiebedingt fehlende erste persönliche Kontakt kann im Studienverlauf oft nicht mehr nachgeholt werden. Hier schließen sich Lerngemeinschaften zusammen und hier geschieht eine erste Sozialisation im neuen Lebensumfeld. Die Studienbeginner*innen brauchen den sozialen Austausch, um sich die neue Lern- und Lebenswelt erschließen zu können und sich mit der Hochschule und ihrer neuen Situation zu identifizieren. Ein weiterer wichtiger Punkt in diesem Zusammenhang ist die Entwicklung eines jeweils unterschiedlichen Fachhabitus. Dieser trägt im späteren Beruf dazu bei, sich mit dem eigenen Berufsbild zu identifizieren und als dazugehörig wahrgenommen zu werden. Diese habituelle Prägung kann kaum digital geschehen. Ein wesentlicher Impuls ist hier, dass für diese Art der Persönlichkeitsentwicklung der persönliche Kontakt in Präsenz unbedingt herzustellen ist.

\subsection{Leitmotive in der Dimension Strukturation}

Im Sinne der Giddens'schen Dualität (s. Kapitel 7.1) stehen Handeln und Struktur in einer engen Beziehung. Durch dieses Wechselspiel und den dadurch entstehenden inkrementellen Prozess (s. Abbildung 27) entsteht Strukturation. In diesem Kapitel sind die Akteur ${ }^{*}$ innen nicht ausgeklammert, da sie diejenigen sind, die sich handelnd Struk- 
tur erarbeiten. Im Vergleich zum vorangegangenen Unterkapitel stehen im Folgenden Motive im Vordergrund, die stärker auf die strukturbildende Ebene als auf die individuelle Handlungsebene abheben. Folgende Leitmotive (s. Abbildung 31) sind in diesem Kapitel wesentlich: Handeln unter Unsicherheitsbedingungen erfolgte in den bisherigen Corona-Semestern in weitaus stärkerem Maße als im Regelbetrieb. Die Akteur*innen mussten innerhalb des Korridors der Strukturation permanent lernen, die neuen Strukturen zu interpretieren, sich anzueignen und in ihnen zu handeln. Es geht also um Kompetenzentwicklung im systemischen wie im individuellen Sinne. Das ergänzende Motiv ist die wiederkehrende Thematisierung der Vorteile sowohl der Präsenz als auch des Digitalen. In der Erarbeitung dieses Zusammenspiels liegt ein wesentliches inkrementelles Moment.

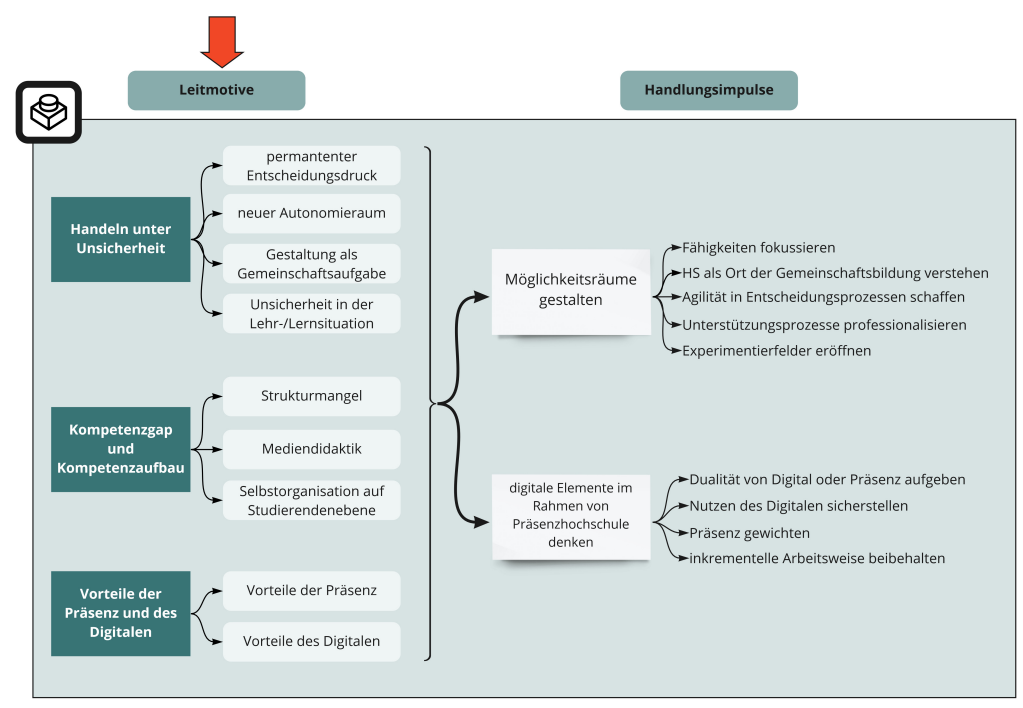

Abbildung 31: Leitmotive in der Dimension Strukturation 


\subsubsection{Handeln unter Unsicherheit}

Permanenter Entscheidungsdruck: Der permanente Entscheidungsdruck ist einer, wenn nicht der zentrale Punkt während der Pandemie. Hochschulleitungen, Lehrende und Studierende waren in einen Zustand hineingeworfen, in dem niemand wusste, was gilt, wie er ${ }^{\star}$ sie sich am besten verhalten soll und was von Dauer und Bestand ist. Hinzu kam die notwendige Ad-hoc-Reaktion, die keine Zeit zu ausgiebiger, strategischer Planung ließ. Aus dem Datenmaterial wurde ersichtlich, dass HAWs mit dieser Situation unterschiedlich umgingen. Grundsätzlich wurde diese Situation am Anfang der Pandemie schon vielfach beschrieben und war in ihrer Gestalt womöglich das bisher herausforderndste Moment der Pandemie. Das Motiv des Handelns unter Untersicherheit begleitete die Hochschulen auch noch weiterhin und setzte sich durch das bisherige Pandemiegeschehen fort. Schließlich mündet die Unsicherheit in die strategische Überlegung, wie Hochschulen in Zukunft gestaltet werden sollen. Diese Unsicherheit zeigt sich in vielen Aspekten. Auf der Ebene der Hochschulleitung ging es zu Anfang der Pandemie um die Aufgabe der Aufrechterhaltung des Studienbetriebs. Hier standen zunächst Satisfizierungs- und nicht Optimierungslösungen im Vordergrund. Rahmenbedingungen - sowohl rechtliche als auch politische - waren und sind vielfach nicht geklärt. Es war zunächst notwendig, Kommunikations- und Entscheidungsprozesse neu zu organisieren und ggf. umzugestalten, um Planungssicherheit zumindest in einem begrenzten Maße gewährleisten zu können. Hier waren viele inkrementelle Prozesse zu beobachten, was unter anderem daran liegt, dass die rechtliche Grundlage an sich durch die wiederkehrenden, nur kurz gültigen Verordnungen auch bereits inkrementell war. Das heißt, dass Hochschulen innerhalb des Kontingenzraums mit vielen Möglichkeiten konfrontiert waren und sich ständig neu ausrichten mussten. Auf diese Weise wurde neue Struktur in den Hochschulen geschaffen. Die Notwendigkeit, innerhalb des Kontingenzraums zu agieren, hat immer wieder neue Handlungen der Akteur*innen der Hochschule hervorgerufen. Die so entstandene Struktur wurde stetig hinterfragt, bestätigt, erneuert oder verändert. 
Hochschulleitungen standen dabei vor der Herausforderung, selbst in der Rolle des Change-Makers Entscheidungen treffen und Themen priorisieren zu müssen, ohne ausreichend Zeit für strategische Überlegungen zu haben. Dabei wurden die eigenen Entscheidungen immer wieder in Frage gestellt. Das Ringen um die richtige Entscheidung zeigte, dass die Notwendigkeit zur Handlung zwar einen Druck erzeugt hat, die Handlungen an sich aber nicht nur reaktiver Natur waren. Hier entstanden emergente Strategien für den Umgang mit einer Vielfalt an Möglichkeiten im Kontingenzraum. Das Ringen um die richtigen Entscheidungen und die dadurch zum Ausdruck gebrachte Unsicherheit sind aber nicht nur Aspekte, die den Anfang des Pandemiegeschehens betreffen, sondern sie zogen sich durch die bisherigen Corona-Semester durch, da die Notwendigkeit der Entscheidungsfindung bei unterschiedlichen Tatbeständen immer wiederkehrte. Diese unterschiedlichen Tatbestände ließen Prozesse verschiedenster Art iterativ werden. Dennoch gelang es den Hochschulen, diese Aufgabe zu bewältigen. Es wurde vermehrt von der Notwendigkeit berichtet, mutige und frühzeitige Entscheidungen zu treffen.

Neuer Autonomieraum: Durch die Pandemie eröffnet sich ein neuer Autonomieraum. Es zeigt sich aber auch ein durch die letzten Jahrzehnte kultivierter Charakter von Hochschulen. Auch wenn das Bild von Hochschulen, die durch restriktive rechtliche Rahmenbedingungen und diverse Kontrollorgane des öffentlichen Rechts sowie den immer wieder neu zu verhandelnden bzw. die zu erringende Finanzstruktur zu sehr simplifiziert ist, stellt sich die Frage, in welcher Wechselwirkung Hochschulen zur übergeordneten Landesstruktur stehen. Die Relevanz dieses Verhältnisses hat sich in der Pandemie erneut deutlich gezeigt. In den Interviews war zu erkennen, dass sich Hochschulleitungen, trotz der Herausforderungen im unsicheren Kontingenzraum, eigenverantwortlich neue Strukturen erarbeiteten und damit ihren Autonomieraum nutzten (s. Kapitel 7.3). Dies gibt also in erster Tendenz die Antwort auf die Frage, ob Hochschulen in diesem Zustand der Unsicherheit in der Lage waren, das fehlende regulatorische Moment insofern zu nutzen, dass sie es in Autonomie umsetzen konnten. Dass die Hochschulangehörigen den Studienbetrieb nicht nur aufrechterhielten, sondern auch - und zwar im Laufe des Pandemiegeschehens zunehmend - in den Blick nahmen, welche strategischen und zukunftswei- 
senden Entscheidungen getroffen werden mussten, zeigt, dass sie von einem reaktiven Verhalten in ein aktives Verhalten gelangen, indem sie die Identität der eigenen Hochschule hinterfragten und im Sinne eines emanzipatorischen Prozesses neue Gestaltungsspielräume eröffneten.

Gestaltung als Gemeinschaftsaufgabe: Als Gelingensbedingung wurde die Gestaltung der Hochschule als Gemeinschaftsaufgabe identifiziert, die über die Einbindung von Gremien an Hochschulen hinausgeht. Es wurde berichtet, dass durch den engen Einbezug der Fachbereiche und Fakultäten Entscheidungsprozesse gemeinsam getroffen werden konnten. Dazu wurden Strukturen aufgebaut oder ergänzt, die neben den regulären Gremienstrukturen eine schnelle und direkte Beteiligung der Akteur*innen und damit einen agilen Entscheidungsprozess ermöglichten. Die Partizipation der Studiengänge gewährleistete in diesem Sinne eine unmittelbare Anbindung an die Lehrenden.

Unsicherheit in der Lehr-/Lernsituation: Die Unsicherheiten betrafen auch die konkrete Lehr-/Lernsituation. Am Anfang des Pandemiegeschehens wurden Unsicherheiten sichtbar, als es um die grundsätzliche Frage ging, wie die Lehre aufrechterhalten werden soll. Die Notwendigkeit zum Handeln brachte hier eine Vielfalt an Fähigkeiten der Akteur ${ }^{\star}$ innen zutage (s. Kapitel 7.2). Die Unsicherheit zog sich dann durch das Pandemiegeschehen fort und nahm aufseiten der Lehrenden mit einer Neuausrichtung der eigenen Rolle als Lehrende ${ }^{\star} r$ Gestalt an verbunden mit der Überprüfung der bisherigen Lehre. Bei den Studierenden richtete sich der Ausdruck der Unsicherheit weniger auf die Ebene der Entscheidungsfindung, sondern bezog sich darauf, wie sie sich in ihrer Rolle verhielten. Gerade die Studierenden waren und sind gezwungen, situativ so zu agieren, dass sie sich im vorgegebenen Rahmen bewegen, da sie durch die hochschulischen Strukturen am wenigsten die Möglichkeit zur Gestaltung der Situation hatten. Auch hier tauchte verstärkt der Aspekt der Regulation auf, der sich darin manifestierte, dass zumindest am Anfang der Pandemie Partizipation an den Veränderungsprozessen durch die Dringlichkeit kaum möglich war. Studierende verlangen aber vermehrt nach dem Recht auf Partizipation, um selbst in der Unsicherheit eine gestaltende Rolle einzunehmen. Auch für Studierende öffnete sich ein Kontingenzraum, in dessen Rahmen es galt, sich funktionierende Strukturen (zurück) zu erobern. 


\subsubsection{Kompetenzgap und Kompetenzaufbau}

Strukturmangel: Der erste wesentliche Aspekt bezieht sich ganz konkret auf den Strukturmangel der Digitalisierung an Hochschulen. Hochschulen haben in vielen Bereichen keinen innovativen digitalen Ausstattungsstandard. Es wird allgemein breit diskutiert, dass die Digitalisierung an Bildungseinrichtungen in Deutschland längst nicht (flächendeckend) auf einem Stand ist, der notwendig wäre, um digitale Lehr-/Lernkonzepte umzusetzen. Förderprogramme der Vergangenheit weisen darauf hin, dass das Problem präsent ist. Die Ergebnisse der Interviews bestätigen diesen Eindruck. Viele Hochschulleitungen, Studierende und Lehrende berichten von einer Lücke in der Infrastruktur, die zwar durch die Pandemie schneller und intensiver bearbeitet wurde, als es ohne das Infektionsgeschehen der Fall gewesen wäre, die aber noch lange nicht geschlossen ist.

Mediendidaktik: Der zweite Aspekt fokussiert den notwendigen Aufbau an Know-how in der Mediendidaktik vonseiten der Lehrenden. Der Bereich der Mediendidaktik an Hochschulen wurde vor der Pandemie lediglich von einigen wenigen Lehrenden anvisiert, die neue digitale Lehrformate ausprobierten. Diese hochschuldidaktischen Stellen waren in den Corona-Semestern gefragter denn je. Es gab folgerichtig nicht nur ein Gap hinsichtlich der technischen Ausstattung, sondern es war auch ein Zuwachs an didaktischem Know-how nötig, um digitale Lehre sinnvoll umzusetzen. Dieses Kompetenzgap wurde im Pandemieverlauf immer weiter geschlossen. Ein großer Teil geschah durch den bereits beschriebenen, inkrementellen Prozess, der als Trial and Error beschrieben werden kann.

Selbstorganisation auf Studierendenebene: Der dritte Aspekt nimmt die Studierenden in den Blick und bezieht sich auf die Notwendigkeit zur Selbstorganisation. Vor der Pandemie wurde nach einem modularisierten und gut durchstrukturierten Studium studiert. Durch die Umstellung wurden die Kompetenzen zu Selbstmanagement und Selbstmotivation sowie die Fähigkeit, digitale Medien nicht nur im privaten Lebensbereich einzusetzen, gefordert wie nie zuvor. Die Erfahrungen aus dem Bereich der Weiterbildung zeigten, dass Elemente des Fernstudiums zu großen Herausforderungen für Lehrende und Studierende führten. Studierende standen vor der Herausforderung, 
keinen konkreten Bezugspunkt zu Kommiliton*innen zu haben und sich selbst hinsichtlich ihres Lernfortschritts selbstständig bewerten zu müssen. Auch in der hier befragten Gruppe zeigten sich alle bereits in Kapitel 1 genannten Herausforderungen von digitalem Lernen. Und genau an dieser Stelle gibt es ein Kompetenzgap seitens der Studierenden. Durch diese Herausforderung war es möglich, Stück für Stück Sicherheit im Umgang mit der eigenen Bildungsbiografie zu gewinnen und mehr eine aktive, gestaltende Rolle einzunehmen. Die Fähigkeit, sich selbst in diesem erforderlichen Maße zu organisieren und darüber hinaus auch Handlungen im Bildungsweg entsprechend zu planen, kann nicht von heute auf morgen erlangt werden, sondern wird durch einen iterativen Prozess erschlossen. So werden Lernstrategien identifiziert, erprobt, im nächsten Zuge ggf. verbessert, angepasst, beibehalten oder ganz verworfen. So schließt sich das Kompetenzgap durch Strukturation inkrementell.

\subsubsection{Vorteile der Präsenz und des Digitalen}

Vorteile der Präsenz: Quer durch alle Zugänge hindurch wurden in den Interviews wiederkehrend Vorteile der Präsenz diskutiert (s. Kapitel 4, Kapitel 5 und Kapitel 6). Als ein wesentlicher Aspekt werden hier genau die Themen adressiert, die mit sozialem Lernen überschrieben werden können und im direkten Zusammenhang zum Sozialisationsprozess der Studierenden an der Hochschule stehen. Der bereits in den vorhergehenden Leitmotiven erwähnte Faktor der persönlichen Interaktion spielte hier eine zentrale Rolle. Nur durch das persönliche Miteinander an einem gemeinsamen Lernort kann ein $\mathrm{Zu}$ sammengehörigkeitsgefühl entstehen, das identitätsbildend wirkt. Für Studierende ist das Studium nicht nur eine Phase intensiven Lernens und der eigenen fachlichen Professionalisierung, sondern auch ein persönlichkeitsbildendes Moment. Diesen Prozess der Sozialisation kann Hochschule am besten in Präsenz leisten. Das Leben an der Hochschule schafft Atmosphäre, bringt Menschen zusammen und ist ein ganz entscheidender Faktor bei der Persönlichkeitsentwicklung der Studierenden (s. Kapitel 1). Diese soziale Einbettung lässt sich digital nur sehr schwer herstellen. Das zeigte die Analyse der Daten und muss 
für die zukünftige Pfadentwicklung berücksichtigt werden. Neben dem Faktor der sozialen Eingebundenheit werden im kollaborativen Miteinander im Lehr-/Lerngeschehen wichtige fachspezifische, habituelle und methodische Kompetenzen erworben. Hier sind es nicht nur die natur- und ingenieurwissenschaftlichen Fächer, die Laborpraxis in Präsenz benötigen, sondern auch die Geistes- und Sozialwissenschaften sind auf die Form der physischen Interaktion angewiesen.

Praktische Fähigkeiten ließen sich am besten in Präsenz lehren und erlernen, so die Befragten in den Interviews und Fokusgruppen. Diese Fähigkeiten betreffen vor allem die HAWs und verwandte Hochschularten, da hier im Vergleich zu theoriebasierten Fächern der Praxisbezug im Vordergrund steht. HAWs zeichnen sich dadurch aus, dass die Studierenden auf Themen treffen, die einen konkreten Bezug zur beruflichen Praxis haben oder einen Input aus dem praktischen Kontext geben. Dieses praktische Lernen geschieht in den Praxissemestern in der Regel direkt in Unternehmen, aber auch an der Hochschule in den Praktikumsräumen und den Laboren. Dabei liegt einerseits der Fokus auf der konkreten Ausübung der praktischen Vorgänge und andererseits auf der Bildung einer anwendungsorientierten Handlungskompetenz, die die Studierenden für ihre zukünftige berufliche Tätigkeit befähigt.

Bei der Pfadentwicklung der Hochschulen nach der Corona-Pandemie, müssen diese Vorteile der Präsenz Berücksichtigung finden. HAWs können durch die zentralen, vorteilhaften Merkmale der Präsenz der Interaktion, Kollaboration und dem praxisnahen Lernen - ihre Profile schärfen. Auf didaktischer Ebene muss diskutiert werden, wie zukünftige Formate der Interaktion und Kollaboration weiterentwickelt werden. Zum Beispiel steht die Frage danach, wie Klein- und Kleinstgruppenarbeit im jeweiligen Fachkontext aussieht und wie die räumlichen Gegebenheiten dafür gestaltet werden müssen, im Fokus.

Vorteile des Digitalen: Trotz aller Themen, die im Digitalen nur schwer umsetzbar sind, wurden auch ganz klare Vorteile des Digitalen herausgearbeitet. Alle Akteur*innengruppen formulieren hier in den Interviews entsprechende Vorteile, die während der Pandemie erkannt und genutzt wurden. Diese beziehen sich auch verstärkt auf organisationale Bereiche. Die durch die Digitalisierung ermöglichte 
Flexibilisierung von Studium und Lehre ist keine neue Idee, denn das Verhältnis zwischen digitaler Lehre und Flexibilisierung hinsichtlich räumlicher oder zeitlicher Strukturen wurde vielfach diskutiert (s. Kapitel 1). Jedoch erleben genuine Präsenzhochschulen diesen Flexibilitätsgewinn erstmals konkret. Sowohl Studierende als auch Lehrende berichten von den Vorteilen, welche die räumliche und zeitliche Unabhängigkeit mit sich bringt. Dazu kommen ebenso Aspekte der zeitlichen Flexibilisierung durch asynchrone digitale Lernformate.

Des Weiteren gibt es ganz konkrete Lehr-/Lernformate, die im Zuge der Digitalisierung der Lehre durchaus positiv bewertet werden. Dazu gehört beispielsweise das Modell des Inverted Classroom oder die Bereitstellung von Lernvideos und anderem Lernmaterial. Kollaborative Verfahren, um gemeinsam an Dokumenten zu arbeiten, sind ebenso positiv bewertet worden wie die unkomplizierte Möglichkeit, externe Lehrende einzuladen oder zusätzliche Fragestunden anzubieten.

Hervorzuheben ist, dass der Nutzen verschiedener Formate sowohl vonseiten der Lehrenden als auch aus der Perspektive der Studierenden unterschiedlich bewertet wurde (siehe s. dazu die genauen Ausführungen in Kapitel 5). Das lässt verschiedene Schlussfolgerungen $\mathrm{zu}$ : Zum einen scheint nicht jeder Unterrichtsgegenstand für jedes Format geeignet zu sein. Es bestätigt zum Beispiel, dass die Methode, auch der Einsatz digitaler Methoden, dem jeweiligen Lernziel angepasst sein muss und sich nicht zwangsläufig jeder Lerngegenstand zur Digitalisierung eignet. Außerdem liegt ein großer Unterschied zwischen der vollständigen Digitalisierung einer Lehrveranstaltung und der Digitalisierung einzelner Bestandteile. Da in der Corona-Situation die Lehre komplett digitalisiert werden musste, konnten nicht nur einzelne digitale Elemente didaktisch sinnvoll eingebaut werden. Die Notwendigkeit, die Lehre in ihrer Ganzheit in digitale Formate umzusetzen, lässt keinen Freiraum zur didaktisch sinnvollen Platzierung von digitalen Elementen. Zum anderen bewerten die Studierenden die Qualität der Lehre unabhängig vom Format unterschiedlich. Es ist $\mathrm{zu}$ vermuten, dass neben dem subjektiven Empfinden der einzelnen Studierenden auch die Qualität der Umsetzung der digitalen Elemente an vielen Stellen noch Potenzial bietet. Die zunächst durchgeführte Eins-zu-eins-Umsetzung der Präsenz ins Digitale beschreibt einen restaurativen Prozess. Hier wurde durch den im Kontingenzraum ausge- 
übten Druck eine Handlung erzwungen, die zunächst satisfizierenden Charakter hatte. Um diesen Status in einen Optimierungsprozess zu überführen, wurden Lehrveranstaltungen nach dem ersten bzw. zweiten Corona-Semester weiter überarbeitet, verbessert und angepasst. So entsteht ein neues Strukturmoment. Die Frage, wie sich diese in der Hochschule entstehenden einzelnen Strukturteile dann zu einem gesamten Bild und damit zu einer Hochschulstrategie zusammensetzen lassen, ist eine der wesentlichen Fragen der Pfadentwicklung.

\subsection{Handlungsimpulse in der Dimension Strukturation}

Das Motiv des Handels unter Unsicherheit zeigte, dass Personen im professionellen Umfeld gerade dann nach Planungssicherheit verlangen, wenn der Kontingenzraum viele Möglichkeiten bietet. Gleichzeitig wurde dieser Möglichkeitsraum als Gelegenheit dafür erkannt, Neues auszuprobieren. Dieses Spannungsfeld ist in der Pandemie besonders stark zutage getreten. Im Sinne eines Muddling-Through-Prozesses schafften es die Hochschulen, sich bei unsicheren Rahmenbedingungen neu zu erfinden. Zunächst ging es um die Aufrechterhaltung des Studienbetriebs. Dazu waren in den Interviews und Fokusgruppe erste emergente Strategien im Rahmen der Strukturation zu erkennen. Diese drückten sich dadurch aus, dass Akteur*innen in den Hochschulen immer mehr von der Reaktion zur Aktion kamen. Die inkrementellen Prozesse wurden gezielter betrieben und Handlung geschah nicht mehr (nur) aus dem Imperativ der Notwendigkeit heraus. Aus den zuvor beschriebenen Motiven wurden daher die Handlungsfelder „Möglichkeitsräume gestalten“ und „digitale Elemente im Rahmen der Präsenzhochschule denken“" abgeleitet (s. Abbildung 32).

Übergeordnet ist die Frage danach, wie sich Hochschulen zwischen Umweltoffenheit und notwendiger Eigenraumbildung verhalten. Durch die Pandemie geschieht Entgrenzung auch an den Außengrenzen der Hochschulen; das System wird durchlässiger. Dennoch ist es für die Hochschulen relevant, das Verhältnis zwischen Umwelt und Eigenraum zu definieren bzw. zu gestalten, wie der eigene Eigenraum aussehen soll. 


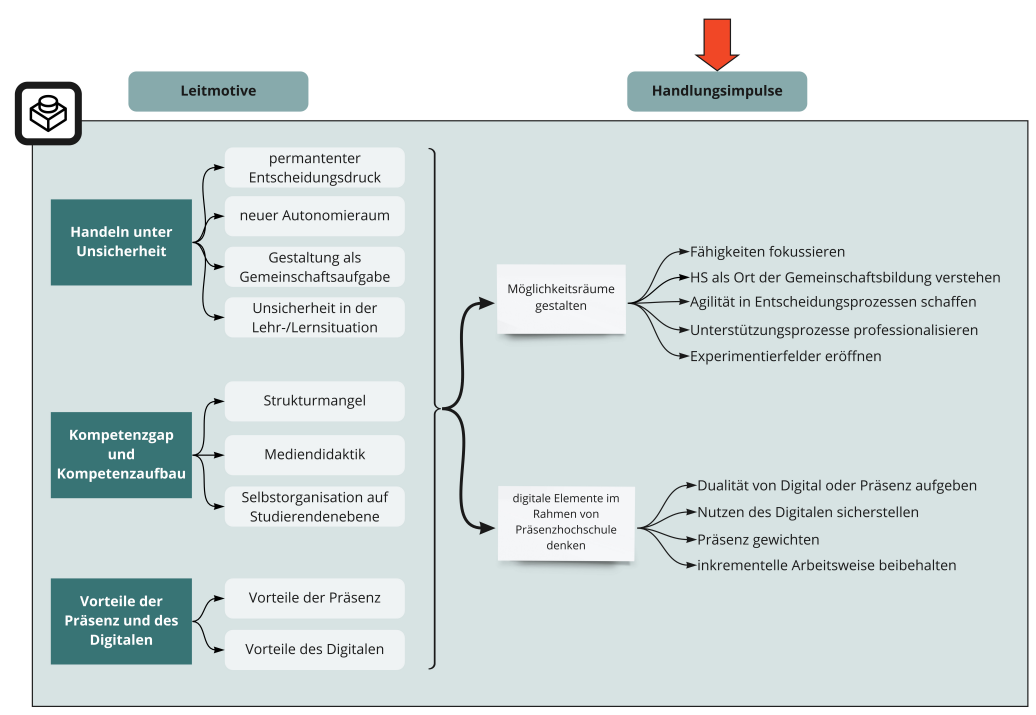

\section{Abbildung 32: Handlungsimpulse im Bereich Strukturation}

Es stellt sich die Frage, wie Hochschulen die Unsicherheiten überwinden und wie die bestehende Kompetenzlücke geschlossen werden konnte. Ein wesentliches Moment liegt in dem „Experimentieren an sich“. In der Unsicherheit der Situation war es erlaubt, Dinge auszuprobieren, zu scheitern, wieder von vorne anzufangen und so iterativ einen Arbeitsmodus zu finden. Hier wird das inkrementelle Moment der Strukturation deutlich sichtbar, welches den einzelnen Akteur ${ }^{*}$ innen viel Energie, Geduld und Ausdauer abverlangte. Es führte aber auch dazu, dass Prozesse breiter vorangetrieben wurden, als dies im Normalzustand der Fall gewesen wäre. Dies betraf auch die Entscheidungsprozesse der Hochschulen. Diese Offenheit drückt eine Haltung aus, die nach vorne gerichtet ist und die es ermöglicht, trotz aller Wünsche nach Planungssicherheit in der Unsicherheit zu wachsen. 


\subsubsection{Möglichkeitsräume gestalten}

Unter Rückgriff auf die in der Einleitung (s. Kapitel 1) dargestellte Grafik wird der sich durch die Pandemie eröffnende Kontingenzraum als Möglichkeit verstanden. Es spannt sich hier ein Raum auf, der durch die wegfallenden oder unklaren Rahmenbedingungen erst entsteht. Er ist angefüllt mit einer Vielzahl von Möglichkeiten des Handelns im jeweiligen hochschulischen Kontext - sei es der Lehre, dem Lernen oder der Hochschulentwicklung und all ihrer Akteur*innen, diesen Möglichkeitsraum zu gestalten und dadurch ein zukunftsfähiges Bild zu entwickeln. Die Hochschulen begeben sich vermehrt auf die Suche nach der geplanten Strategie post Corona. Diese Pfadentwicklung kann nur durch die gestalterische Leistung der hochschulischen Akteur*innen gelingen.

Fähigkeiten fokussieren: Es zeigte sich an vielen Stellen, dass die Akteur ${ }^{\star}$ innen im Rahmen ihrer Fähigkeiten strukturbildende Arbeit leitsteten. Nicht nur die Hochschulleitungen, Lehrenden und Studierenden, sondern auch andere Funktionsträger*innen wie die Fakultätsleitungen oder Beschäftigte in den Abteilungen leisteten während der Pandemie hervorragende Arbeit - dies wurde in vielen Interviews und Fokusgruppen betont. Hervorgehoben wurde auch, dass diese Aufgabe nicht von einer Stelle alleine bewältigt werden konnte, sondern dass es nur im Zusammenspiel aller Beteiligten möglich war. Daher besteht ein Handlungsimpuls darin, dass für die zukünftige Pfadentwicklung die Fähigkeiten und die Mündigkeit der Akteur*innen stark fokussiert werden. Die nun benötigten Kompetenzen sind nicht mehr zwangsläufig die, mit denen die Tätigkeit an der Hochschule aufgenommen wurde. Es wurden neue kollaborative Arbeitsweisen entwickelt, in denen Entscheidungen und Abläufe in einem iterativen Prozess miteinander verhandelt wurden. Akteur*innen müssen demzufolge zukünftig an ihren Hochschulen neben professionalisierter Fachkompetenz vor allem eine andere, flexible Haltung einnehmen, um den zukünftigen Anforderungen $\mathrm{zu}$ genügen. Eine zentrale Anforderung an die Tätigkeit an einer Hochschule ist die Fähigkeit, sich selbst in seinem Berufsbild zu reflektieren und daraus zukunftsfähige Handlungen abzuleiten. Gleichzeitig muss die eben erwähnte Fachkompetenz erweitert und verändert werden. Durch die Digitalisierung wird ein vergrößerter 
Methodenkoffer in der Lehre und zudem erweiterte digitale Kompetenzen den jeweiligen Arbeitsgegenstand betreffend gefragt. Sich selbst in die Lage zu versetzen, agil im eigenen und neuen Arbeitsumfeld zu agieren, wird auch von wesentlicher Bedeutung sein.

Hochschule als Ort der Gemeinschaftsbildung verstehen: Zudem ist es relevant, die Hochschule nicht nur als Bildungseinrichtung, sondern als Ort der Gemeinschaftsbildung zu verstehen. Unter der Pandemie tritt dieser Aspekt in den Hintergrund, da sich durch die physische Ferne zum Campus nur bedingt ein Gefühl der Zugehörigkeit herstellen lässt. An welcher Hochschule studiert oder gearbeitet wird, tritt in den Hintergrund, da im Homestudying, Hometeaching oder Homeoffice die Zugehörigkeit zu einer bestimmten Hochschule kaum Relevanz besitzt. Es ist daher umso wichtiger, ein Gefühl der Zugehörigkeit zu schaffen, das zur Identifikation mit der eigenen Hochschule führt. Für die Studierenden ist zudem die Zugehörigkeit zu ihrer Kohorte und ihrem Fach von entscheidender Bedeutung. Das Knüpfen von Freundschaften gestaltet sich im Digitalen jedoch schwer. Daher wurden gezielte Maßnahmen und Angebote eingerichtet, um neben der konkreten Arbeitssituation im Meeting oder der Vorlesung ein Aufeinandertreffen auch digital zu ermöglichen.

Agilität in Entscheidungsprozessen schaffen: Die Agilität, d. h. die Wandlungsfähigkeit, sollte auch in die Entscheidungsprozesse implementiert werden. Gerade in der Corona-Situation hat sich gezeigt, wie wichtig Agilität auf der Ebene des Hochschulmanagements ist. Warum ist Agilität aber nicht nur während der Pandemie sondern auch für zukünftiges Handeln der Hochschulen hilfreich? Im agilen Verhalten liegt ein gewisses Maß an Innovationsfähigkeit. In agilen Entscheidungsprozessen während der Pandemie wurden kreative Lösungen gefunden und umgesetzt, die sonst an der Trägheit etablierter Strukturen gescheitert wären. Post Corona kann diese Agilität genutzt werden, um Gestaltungspielräume zu identifizieren, Rahmenbedingungen in Frage $\mathrm{zu}$ stellen und Lösungen out of the box zu generieren. Damit steht nicht mehr (nur) die Frage an erster Stelle, was erlaubt wäre, sondern was notwendig für die Zukunftsfähigkeit an Hochschulen ist. Diese agilen Entscheidungsprozesse unterliegen idealerweise einem iterativen Prozess und beteiligen die relevanten Akteur*innen auf Augenhöhe. Beides sind Gelingensbedingungen für transformative Prozesse 
und können dazu beitragen, dass Agilität in Entscheidungsstrukturen in der Realität gelebt werden kann.

Unterstützungsprozesse professionalisieren: Für das Schließen der Kompetenzlücke ist die Professionalisierung von Supportstrukturen wesentlich. Diese Unterstützungsleistungen (z. B. didaktischen oder technischen Support) gilt es $\mathrm{zu}$ professionalisieren und damit an den Hochschulen Keimzellen für (medien-)didaktische Innovation zu schaffen. Dies umfasst auch die Weiterqualifikation von Mitarbeiter ${ }^{\star}$ innen. Eine zweite Perspektive ist die studentische, für die ebenfalls Supportstrukturen aufgebaut wurden. Hier ging es über die konkrete technische Hilfe hinaus und zeigte sich in Programmen zur Begleitung der Studierenden im Homestudying. Diese Programme sollten zukünftig nicht abgesetzt werden, sondern sich zu studierendenzentrierten Unterstützungsangeboten weiterentwickeln, welche über den Fokus des Fernstudiums hinausgehen. Dass digitales Lehren weiteren didaktischen Kompetenzaufbau benötigt, ist allgemein diskutiert, aber auch digitales Studieren muss gelernt werden. Wenn sich Hochschulen zukünftig dafür entscheiden, weiterhin einen Anteil digitale Formate in der Lehre anzubieten, dann ist es auch notwendig, den studentischen Kompetenzausbau in den Blick zu nehmen.

Experimentierfelder eröffnen: Grundsätzlich hat sich als ein wesentliches Gelingenskriterium herausgestellt, dass in iterativen Prozessen nach und nach sowohl Kompetenz als auch Struktur aufgebaut wurde. Schließlich konnten die Hochschulen in den letzten Semestern beweisen, dass Experimentieren unter unsicheren Rahmenbedingungen nicht nur befriedigende Ergebnisse erzielen kann, sondern auch Optimierungsvorgänge in Gang setzt. Daher wird hier als Handlungsimpuls die Eröffnung von Experimentierfeldern genannt. Dieses Experimentieren bezieht sich auf verschiedene Bereiche: So können damit die Lehre, der Umgang mit neuen digitalen Formaten, aber auch der zentrale Bereich und die Kernprozesse der Hochschulen gemeint sein. In diesem Setting des Prototypings kann Innovation entstehen. Schließlich beruht es auf dem Vertrauen, dass in den letzten Semestern die Herausforderungen bewältigt wurden und dass der Gedanke des Experimentierens und Bastelns an Lösungen dabei einen entscheidenden Beitrag leistete. An der traditionell geprägten Institution Hochschule solche Freiräume zu schaffen, ist jedoch eine Herausforderung. 
Dennoch kann hier ein großer Vorteil für die Zukunftsfähigkeit von Hochschulen liegen.

\subsubsection{Digitale Elemente im Rahmen von Präsenzhochschulen denken}

Im vorherigen Kapitel wurden als ein Leitmotiv Kompetenzgap und Kompetenzaufbau ausgeführt. Von Defiziten zu sprechen, erscheint aber nur vor dem Hintergrund eines Referenzrahmens sinnvoll. Warum haben Lehrende und Studierende ein Gap hinsichtlich des Lehrens und Lernen mit digitalen Medien? Die Antwort erscheint einfach: Weil alle Befragten an einer Präsenzhochschule beschäftigt bzw. eingeschrieben sind. Die Hochschulen waren bisher auf Präsenzunterricht ausgerichtet. Mediendidaktische Elemente wurden zwar vereinzelt schon genutzt, aber nur, um Lehrveranstaltungen $\mathrm{zu}$ ergänzen und nicht komplett zu ersetzen. Denn der - vielleicht zu hinterfragende oder zu bekräftigende - Charakter der HAWs liegt in ihren Stärken in Form der direkten Studierenden-Lehrenden-Interaktion und dem direkten Praxisbezug. Vor diesem Hintergrund müssen digitale Elemente neu gedacht werden. Die Innovation ist nicht, dass es post Corona Digitalisierung in der Lehre gibt, denn das war vorher schon vereinzelt oder beispielsweise in der wissenschaftlichen Weiterbildung oder an Fernhochschulen der Fall. Die Neuerung ist, dass sich post Corona Präsenzhochschulen, die nunmehr zwei Semester digitale Lehre umgesetzt haben, fragen müssen, welche dieser digitalen Elemente Zugang zu ihrer Hochschul- und Digitalisierungsstrategie findet. Die Herausforderungen der Hochschulen liegt nun genau darin, einerseits nicht in eine alte Normalität, d.h. in denselben Pfad wie vor Corona zurückzufallen, andererseits im Sinne eines transformativen Prozesses zu erarbeiten, wie der gewünschte Zielzustand aussehen kann und damit die Stufe der Restauration zu überwinden.

Dualität von Digital oder Präsenz aufgeben: Zunächst einmal muss die Dualität von Digital oder Präsenz aufgehoben werden. Das sind in vielen Köpfen zwei Welten. Der Gedanke, dass das Beste aus beiden Welten - der digitalen und der Präsenzwelt - gebraucht wird, verleitet dazu, hier eine Dualität von entweder - oder zu denken. Es ist unumstritten, dass die befragten HAWs an ihrer Identität als Präsenz- 
hochschule festhalten. Ein Schwarz-Weiß-Denken würde aber dazu führen, dass damit der digitale Bereich ausgeschlossen wird. Das wäre dann ein Zustand der alten Normalität, auf den die Befragten, wie bereits vielfach gesagt, nicht mehr zurückfallen wollen. Gleichzeitig distanzieren sich die HAWs vom Bild der Fernhochschule und damit vom Gedanken der vollständigen Digitalisierung. Darüber, dass es nicht so bleiben kann, wie es derzeit während des Pandemiegeschehens ist, herrscht Einigkeit. Hochschulen sollten verinnerlichen, dass es zwischen Digitalem und Präsenz ein Kontinuum gibt, das ausgeschöpft werden sollte. Hochschulen werden sich in Zukunft irgendwo in diesem Kontinuum wiederfinden. Die spannenden Fragen sind, wie sich Hochschulen strategisch aufstellen, ob sie ihre Verortung profilbildend nutzen und wie dann ganz konkret das Zusammenspiel zwischen Präsenz und Digitalem gestaltet wird. Das Verlassen des Schwarz-WeißDenkens kann auch auf die einzelne Lehrveranstaltung heruntergebrochen werden. Eine Lehrveranstaltung muss nämlich nicht zwangsläufig in Präsenz oder digital stattfinden. Jeweilige Aspekte in den Lehrveranstaltungen können entlang der Lernziele mit verschiedenen Methoden gestaltet werden.

Nutzen des Digitalen sicherstellen: Fernhochschulen oder die wissenschaftliche Weiterbildung - also Bereiche, die schon länger auf digitale Elemente oder eine vollständige Digitalisierung zurückgreifen - haben einen besonderen Grund, dies zu tun. Dieser liegt in der Notwendigkeit zur zeitlichen und räumlichen Flexibilität, der von berufsbegleitend Studierenden gefordert wird. Diesem Bedarf kann gut durch digitale Formate entsprochen werden. Auch wenn die mögliche Flexibilisierung durch digitale Formate bereits als Vorteil des Digitalen herausgearbeitet wurde, ist es doch für Präsenzhochschulen nicht unbedingt notwendig, diese Flexibilität herzustellen. Die Zielgruppe der Präsenzhochschule sind in erster Linie nicht berufsbegleitend Studierende, sondern Personen, die durch die Wahl einer Präsenzhochschule signalisieren, dass sie durchaus Zeit und Raum haben, zu den Vorlesungen an die Hochschulen zu kommen. Hier kann die Flexibilisierung zwar eine neue Zielgruppe erschließen und zu weiteren Freiheitsgraden bei der Kompatibilität von Privatem und Studium führen, dennoch müssen noch weitere sinnvolle Gründe für die Implementierung von digitalen Elementen in die Lehre an Prä- 
senzhochschulen herangezogen werden, da eine komplette Loslösung von zeitlichen und räumlichen Strukturen das Profil der Präsenzhochschulen aushebeln könnte. Hier muss im jeweiligen Hochschulkontext diskutiert werden, welche Möglichkeiten des Einsatzes von digitalen Elementen im Lehr-/Lernkontext im Kontingenzraum nutzbringend für die zukunftsweisende Pfadentwicklung sind. Auf Ebene der jeweiligen Lehrveranstaltung betrifft dies ganz grundlegende didaktische Fragen hinsichtlich der konkreten Lernziele und der dazu passenden Methodik. Beispielsweise kann sich als nutzbringend erweisen, digitale Elemente zur Wissensvermittlung asynchron zur Verfügung zur stellen, um die Präsenz für Interaktion zu nutzen. In einer anderen Veranstaltung kann möglicherweise die gemeinsame Arbeit an einem Textdokument sinnvoll digital gelöst werden. Die Möglichkeiten sind so vielfältig wie die Lehrveranstaltungen selbst. Als handlungsleitender Impuls soll hier im Speziellen festgehalten werden, dass digitale Elemente, wie jede didaktische Methodik, nicht um ihrer selbst willen, sondern immer vor dem Hintergrund der Rolle der Präsenz und der jeweiligen Fachkonstellation eingesetzt werden können.

Präsenz mehr gewichten: Auch in den Interviews und Fokusgruppen wurde vermehrt die Aussage formuliert, dass Präsenz mehr gewichtet werden sollte. Eine der wesentlichen Erkenntnisse der Untersuchung war, dass HAWs Präsenzhochschulen bleiben wollten, auch wenn sie die Digitalisierung gut gemeistert haben und Hochschule offensichtlich auch (irgendwie) ohne den direkten Kontakt und den Campus funktioniert. HAWs leben von dem direkten Austausch zwischen den Hochschulangehörigen, haben ihre Stärken gerade in der Anwendungsorientierung, dem Praxisbezug und dem praktischen Lernen. Auch durch den pandemiebedingten Digitalisierungsschub können bzw. sollen aus HAWs keine Fernhochschulen werden. Den Hochschulen und ihren Akteur*innen wurde durch die Pandemie deutlich, welchen hohen Stellenwert die Präsenz an Hochschulen hat. Jetzt ist es genau diese bereits vielfach genannte Herausforderung, den Digitalisierungsschub trotzdem zu nutzen, aber weiter ein identitätsbildendes Profil herauszuarbeiten. Bei der Frage, ob die Lehre zukünftig überwiegend digital oder eher in Präsenz stattfindet, würde die Waage zugunsten der Präsenz ausschlagen. Für eine Pfadentwicklung an HAWs soll diese Zustimmung aus den Interviews und Fokusgruppen als Impuls 
dienen, um weiterhin dieses Profil zu schärfen. Gleichzeitig besteht die Notwendigkeit, die gewonnenen Erkenntnisse zu den Vorteilen der digitalen Lehre zu nutzen, um die bestmögliche Lehre an Hochschulen zu gestalten.

Inkrementelle Arbeitsweise beibehalten: Wie kann der Gedanke der Präsenzhochschulen mit den Impulsen zur Digitalisierung in der Lehre verbunden werden? Im Pandemiegeschehen erwies sich die inkrementelle Arbeitsweise als sehr nutzbringend. Dabei wird in einem iterativen Prozess Struktur geschaffen, reflektiert, in einem nächsten Durchgang erprobt, infrage gestellt, verworfen oder verifiziert. Dieser Prozess kann beliebig viele iterative Schritte umfassen. Auch zur Ausarbeitung von Strukturen der zukünftigen Hochschulausgestaltung in den Lehr-/Lernformaten kann dieses iterative Vorgehen gewinnbringend sein, um aus Handlungen Strukturen abzuleiten. Schließlich können so gewonnene, emergente Strategie in die Hochschulstrategie post Corona münden. Vielfach wurde beschrieben, dass die didaktischen Möglichkeiten an Präsenzhochschulen an Vielfalt gewonnen haben. Das ist zunächst eine sehr begrüßenswerte Situation. Das eingangs erwähnte Spannungsfeld zwischen Freiheit der Lehre und Strategie der Hochschule bleibt trotzdem oder gerade deswegen zu gestalten. Die Studierenden und auch die Lehrenden forderten hier eine Verständigung auf ein einheitliches Vorgehen. Eine Einigung wird jedoch kaum zu erreichen sein. Ein grundsätzliches Vorgehen könnte aber hinreichend konkret sein und über einen Leitbildgedanken, dass sowohl Präsenz als auch Digitalisierung möglich ist, hinausgehen. Dass Studierende äußerten, dass die Art und Weise des digitalen Lernens an der jeweiligen Hochschule einen Entscheidungsfaktor bei der Studienplatzwahl darstellt, legt den Schluss nahe, dass die Hochschule bzw. die Fakultäten/Fachbereiche oder Studiengänge dafür Sorge tragen sollten, dass ein profilbildendes Konzept in der Mischung zwischen Digitalem und Präsenz umzusetzen. Innerhalb dieses eben geschilderten Rahmens soll jedoch ein Möglichkeitsraum zum Experimentieren entstehen, der sich durch Flexibilität auszeichnet. So kann der Weg zur strategischen Pfadentwicklung Stück für Stück erschlossen werden. 


\subsection{Leitmotive in der Dimension Strategie}

Wollte jemand die kantische Frage umformulieren, sie könnte heute wohl lauten: „Wie ist ein Neues überhaupt möglich?“ (Adorno, 1976). Alle Hochschulen sind sich einig darüber, dass ihnen eine große $\mathrm{Zu}$ kunftsaufgabe bevorsteht. Wie nämlich soll die Hochschule in der Zukunft gestaltet werden? Die Herausforderung post Corona wird darin liegen, die nötige Aktivierungsenergie aufzubringen und den Zustand der Restauration in eine transformative Pfadentwicklung zu überführen. Denn klar scheint zu sein, dass Hochschulen, die in die alte Normalität zurückfallen, Gefahr laufen, über kurz oder lang im Bildungssystem abgehängt zu werden.

Auf der Basis der Interviews und Fokusgruppen lassen sich zwei wesentliche Leitmotive erkennen, welche konkret strategische Themenbereiche tangieren (s. Abbildung 33).

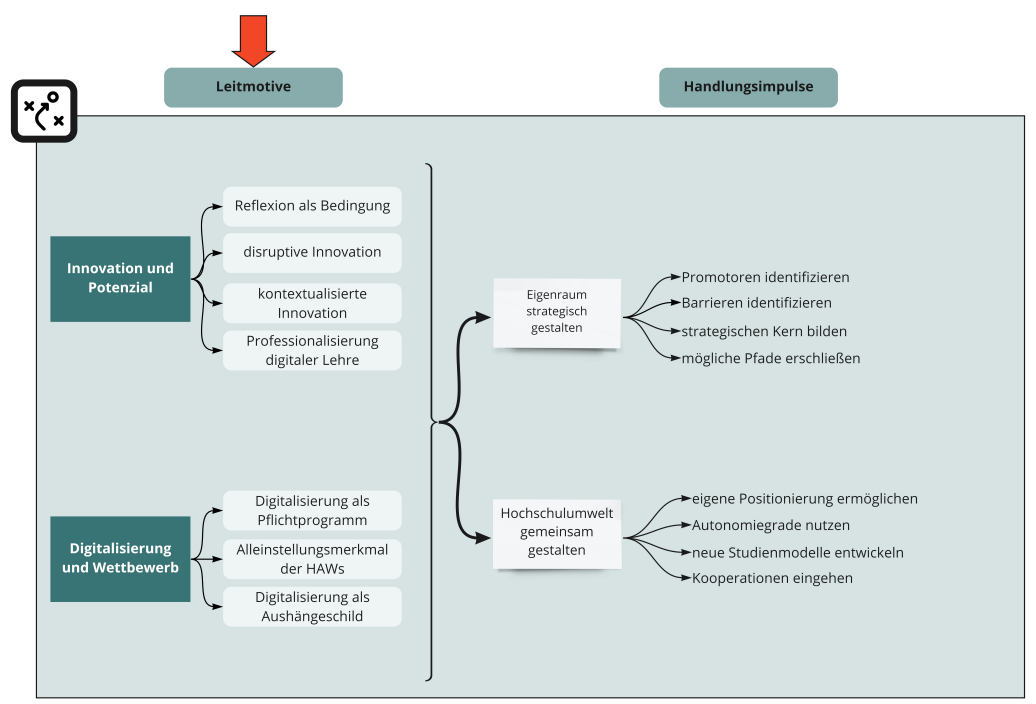

Abbildung 33: Leitmotive in der Dimension Strategie

Das erste Leitmotiv umfasst Aspekte, welche das strategische Thema Innovation und Potenzial adressieren. Es bezieht sich also auf die In- 
novationskraft, die Hochschulen entwickelten, bzw. das Innovationspotenzial, das Hochschulen ausschöpfen können. Ein zweites Leitmotiv bringt Digitalisierung mit Wettbewerb in Verbindung.

\subsubsection{Innovation und Potenzial}

Reflexion als Bedingung: Innovation erfordert Reflexion. Diese hat sich als eine notwendige Bedingung für Innovation als wesentlich für die Bewältigung der Transformation der Hochschulen herausgestellt. Schon das routinierte Alltagshandeln in Hochschulen stößt immer wieder auf Probleme und generiert Lösungen. Daraus resultiert jedoch die Frage, wie sich Neues vom Altem unterscheidet, wie ausgefüllte Handlungsspielräume zu Innovationen werden. Oevermann spricht von der Annahme, dass Neues wahrscheinlich in ersten „Transformationstakten" von sequenzierten Abläufen recht häufig keimhaft entsteht, aber durch die Reproduktionsgesetzlichkeit einer Fallstruktur diese Anfänge einer Transformation wieder in die alte Bahn gelenkt werden. Das Problem wird also sein, wie an keimhaft entstandenem Neuen von der sich transformierenden Lebenspraxis selbst auf Dauer festgehalten werden kann (Oevermann, 1991). Erforderlich dazu ist ein Moment der Reflexion und der Wiederholung. Etwas Neues im Handeln muss von den Akteur*innen bemerkt und aufgegriffen werden, um nicht in den kleinen Abweichungen zu versanden, die schon in der Routine enthalten sind. Dieser Punkt ist zentral: Zur Innovation gehört ein Moment der Deutung und Zuschreibung, des Innehaltens, Bewertens und Aufgreifens. Die Reflexivität des Handelns macht sich an diesem Punkt explizit bemerkbar, und von diesen Deutungs- und Entscheidungsoperationen hängt es wesentlich $a b$, ob eine Abweichung zur Innovation wird. Damit ist die Innovation - wie das Handeln allgemein - auf Intersubjektivität und Anerkennung angewiesen (Oevermann 1991).

Disruptive Innovation: Innovation in dem bisherigen Sinne kann als inkrementell bezeichnet werden. Diese Art der Innovation kennzeichnet weite Bereiche des Handelns unter Corona-Bedingungen. Dies darf jedoch nicht dazu führen, dass disruptive Innovationen aus dem Blick geraten. Disruptive Innovationen bezeichnen nicht ein allmäh- 
liches Hineingleiten in ein Neues, sondern eine Setzung: Das Neue wird an die Stelle des Alten gesetzt. Disruptive Innovation geht oft einher mit geplanter Strategie. Im Rahmen der Untersuchung haben hochschulische Akteur*innen Veränderungen im Hochschulkontext sprachlich als Quantensprünge bezeichnet. Diese Wahrnehmung wird als disruptive Innovation interpretiert. Hochschulen sind im Vergleich zu Fernhochschulen oder international gültigen Standards im Hinblick auf Digitalisierung noch nicht in die Dimension der Innovation vorgedrungen. Dennoch bedeutet der Einsatz von digitalen Elementen in der Lehre in der Breite für die untersuchten Hochschulen einen Strukturbruch und damit ein disruptives Geschehen. Das alte System wurde durch ein neues ersetzt. Dieser Prozess der Ersetzung wurde von den Beteiligten als Innovationsschub interpretiert. So kann etwa in Bezug auf die Gestaltung von Lehrveranstaltungen von Disruption gesprochen werden, da ein etabliertes Vorgehen abrupt ersetzt werden musste. Dieses Verständnis von Disruption basiert auf der Vorstellung einer von außen herbeigeführten, nicht geplanten, sondern erzwungenen Veränderung. Disruption kann auch dergestalt interpretiert werden, dass Veränderung absichtlich herbeigeführt wird und ganz gezielte Maßnahmen eines radikalen Wandels eingeleitet werden.

Kontextualisierte Innovation: Der Begriff Innovation erweist sich in hohem Maße als kontextabhängig. Was bedeutet das? Wenn Lehrende beispielsweise den Einsatz von Videos in der Lehre als Innovation bezeichnen, so ist diese Aussage notwendigerweise in einen Referenzrahmen einzuordnen. Videos, um bei diesem Beispiel zu bleiben, sind grundsätzlich keine neue Erfindung. Seit Langem finden sich auf YouTube im Open Access Video-Tutorials, im Feld der Weiterbildung sind MOOCs ein gebräuchliches Werkzeug, auch die Fernhochschulen arbeiten mit asynchronem Videomaterial. Warum also ist die Aussage, dass in der Lehre eingesetzte Videos für die befragten Hochschulen eine Innovation darstellen, dennoch nicht falsch, wenn es sich dabei doch um ein bereits etabliertes Format handelt? Warum sprechen die Akteur*innen von Innovationen? Die Antwort fordert das Einbeziehen des Kontextes. Im Feld der Hochschulen sind die Präsenzhochschulen der Regelfall. Bis vor einem Jahr wurden digitale Medien nur punktuell und nur unterstützend oder ergänzend von den Lehrenden eingesetzt, welche besondere Affinität zur Digitalisierung hatten und die daher 
schon früh angefangen haben, Digitalisierung in der Lehre zu berücksichtigen. Für die überwiegende Mehrheit der Hochschullehrenden stellt der Einsatz von Videos in der Lehre - also innerhalb ihres Referenzsystems - eine Innovation dar. Das heißt: Die in den letzten zwei Semestern und jetzt im dritten Semester geschehene Innovation muss in der Relation dazu gesehen werden, wie es an Hochschulen noch vor einem Jahr aussah. Dabei lässt sich das Beispiel der Videos auf beliebige andere Bereiche, wie z. B. die Digitalisierung von Verwaltungsabläufen oder die digitale Kommunikation, übertragen.

Professionalisierung digitaler Lehre: Ein wesentliches Innovationsfeld für die Hochschulen liegt aber darüber hinaus in der Professionalisierung digitaler Lehre.

Das, was bereits im letzten Jahr im Bereich der Lehr-/Lernformen erprobt wurde, kann genutzt werden, um als Basis für zukünftige Professionalisierung zu dienen. Es ist nicht möglich, während des laufenden Betriebs alle Lehrveranstaltungen mit innovativen Qualitätsstandards in digitale Formate umzuwandeln. Darum werden bereits bestehende Formate weiter verbessert werden müssen, um den Innovationscharakter zu bewahren und die Formate zielführend einsetzen zu können. In diesem Zusammenhang ist zu diskutieren, wie zeitliche und räumliche Flexibilisierung sowie Individualisierung von Lehre durch digitale Elemente als Innovation genutzt werden sollen. Diese Frage hängt eng damit zusammen, wie das zukünftige Modell der Lehre insgesamt gestaltet wird. Dabei tangiert das Thema der Flexibilisierung nicht nur mikrodidaktische Elemente, sondern es eröffnet auch bei der Studiengangplanung neue Möglichkeiten.

\subsubsection{Digitalisierung und Wettbewerb}

Digitalisierung als Pflichtprogramm: Wird Digitalisierung im Bildungsbereich mit dem Thema Wettbewerb in Verbindung gebracht, ist zunächst festzuhalten, dass Digitalisierung aufgrund der Pandemie nicht mehr nur eine mögliche Option darstellt, sondern vielmehr Pflichtprogramm für Hochschulen geworden ist. Ganz unabhängig von den Grundvoraussetzungen sind alle Hochschulen gezwungen, Lehre digital umzusetzen. Jedoch sind Unterschiede in den Qualitäts- 
standards zu beobachten. So zeigt sich beispielsweise, dass Hochschulen, die in der Lehre das Fach Informatik oder verwandte Fächer vertreten, durch das bereits dadurch an der Hochschule vorhandene Know-how anders an die Digitalisierung der Lehre bzw. der Verwaltungsabläufe herangehen und dass das entsprechende Wissen aus den Fachbereichen in die Hochschule Eingang findet. Andere Hochschulen haben davon berichtet, bisher keine oder kaum Vorerfahrung mit Digitalisierungsprozessen zu haben. Da nun aber alle vor derselben Herausforderung stehen, liegt der Unterschied darin, welchen Weg Hochschulen im Möglichkeitsraum wählen. Das bedeutet aber auch, dass es in der jetzigen Situation so relevant ist wie selten zuvor, herauszuarbeiten, was profilbildende Merkmale an Hochschulen sind, damit Hochschulen auch zukunftsfähig sein können.

Einen wesentlichen Einfluss auf die Wahl des Pfades hat aber nicht nur das entsprechende Know-how, sondern auch die infrastrukturelle Ausstattung mit Blick auf die Technik. Diese spielt auch eine Rolle bei vielen Gestaltungsfragen der Hochschule und kann somit je nach ihrer Qualität zum Wettbewerbsvor- oder -nachteil werden. Neben der Lehre ist auch der Bereich der Verwaltung zu beachten. Hier ist nicht nur die richtige Infrastruktur, sondern auch eine Veränderung der Verwaltungsprozesse relevant. Es muss ein transformativer Ansatz gewählt, damit Prozesse jenseits der Infrastruktur gestaltet werden. Andernfalls laufen die Hochschulen Gefahr, bei digitaler Restauration zu verharren.

Alleinstellungsmerkmal der HAWs: Es ist des Weiteren wichtig, den Wettbewerbsfaktor Digitalisierung mit den Alleinstellungsmerkmalen der HAWs in Einklang zu bringen. In der Befragung taucht regelmäßig das Motiv auf, dass die Alleinstellungsmerkmale der HAWs identifiziert werden müssen. Nicht, um sich generell abzugrenzen und in Konkurrenz $\mathrm{zu}$ treten, sondern vielmehr, um zu bestimmen, welche Position HAWs im Bildungsraum zukünftig besetzen können. Die Digitalisierung hat Hochschulen Kontingenzräume eröffnet und zwingt sie zu klären, wie profilbildende Merkmale von Hochschularten und einzelnen Hochschulen gestaltet werden können.

Digitalisierung als Aushängeschild: Digitalisierung fungiert zudem als Aushängeschild. Infrastruktur und Ausstattung in der Lehre sind nicht nur für eine innovative Ausgestaltung von Lehr-/Lernformaten 
notwendig, sondern repräsentieren auch die Hochschule nach außen. Wenn Studierende mit qualitativ schlechtem, digitalem Material konfrontiert werden, ist das kein gutes Aushängeschild der Hochschulen. Wenn leistungsbezogene Indikatoren, wie die Auslastung der Studienplätze, Studienabbruch oder Drop-out-Quote, zur Steuerung von Hochschulen herangezogen werden, kann Digitalisierung ein Wettbewerbsfaktor werden. Die Pandemie hat den Digitalisierungsstandards größere Bedeutung verliehen. Was vor einem Jahr noch nicht von entscheidender Bedeutung war, ist es möglicherweise heute. Die Studierenden bringen zum Ausdruck, dass die Art des digitalen Lehr-/ Lernkonzepts für sie eine Rolle bei der Wahl des Studienorts spielt. Das muss Hochschulen alarmieren und dazu bringen, Digitalisierung in ihre strategische Pfadentwicklung einzubeziehen. Hinzu kommt, dass die Studierenden der Zukunft in ihrer Schullaufbahn nun schon über ein Jahr (mehr oder weniger ausgeprägt) mit digitalem Lernen konfrontiert waren. Sie verfügen also unter Umständen auch über andere Kompetenzen als Studienbeginner*innen es bisher taten. Sie sind ggf. auch dazu in der Lage, digitale Lehre differenzierter zu bewerten. Dieser Aspekt lässt sich von der Lehre auf die Verwaltung übertragen. Für Hochschulen der Zukunft kann es kein Aushängeschild sein, wenn eine Immatrikulation mit großen Papierstapeln einhergeht. Hier sind die Hochschulen gefragt, ihr Konzept so auszurichten, dass es sie nach außen repräsentiert.

\subsection{Handlungsimpulse in der Dimension Strategie}

Die Hochschulen befinden sich in einer Situation zwischen digitaler Restauration und Transformation - digitale Restauration in dem Sinne, dass bereits Vorhandenes durch die „Bearbeitung der Fassade“ in ein neues, digitales Format umgewandelt wurde. Im Rahmen inkrementell verlaufender Prozesse entwickeln sich emergente Strategien. Hochschulische Strategien vor der Corona-Pandemie wurden aufgrund des entstandenen Handlungsdrucks in den Hintergrund gedrängt oder gar gänzlich abgelöst.

Welche Schlüsse kann man also für die Strategieformulierung und die entsprechende Pfadentwicklung auf der Grundlage der in Kapitel 7.6 
dargestellten Leitmotive ziehen? Ausgehend von dieser Analyse wurden zwei Handlungsfelder als wesentlich herausgearbeitet.

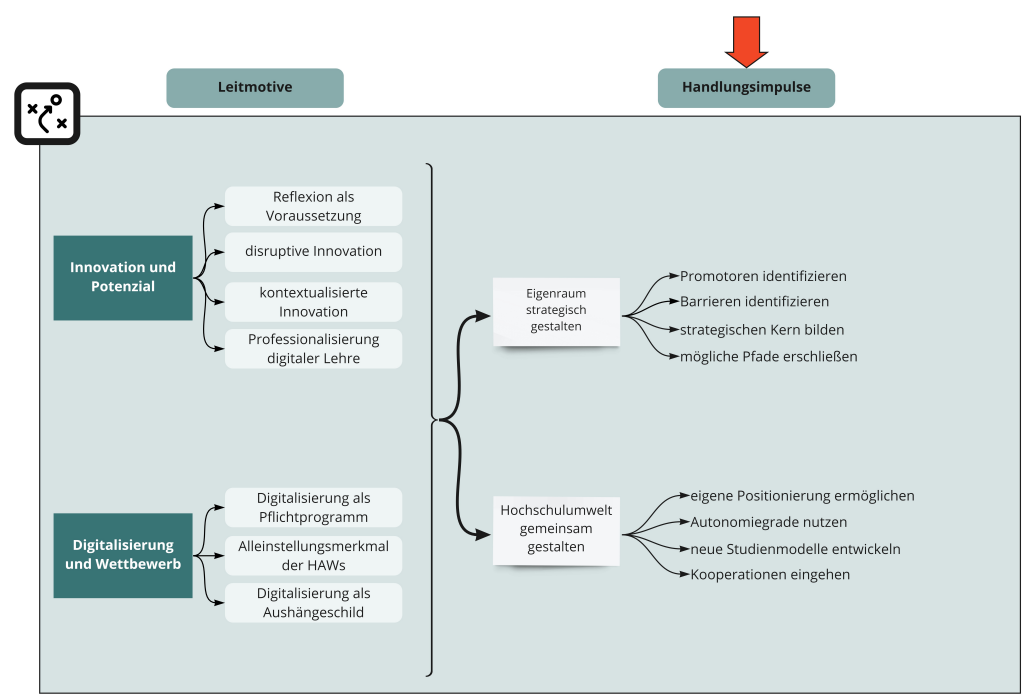

Abbildung 34: Handlungsimpulse in der Dimension Strategie

Da es (s. Abbildung 2) mehrere mögliche Pfade hin zu einer Hochschulstrategie post Corona geben kann, können hier auch nur Handlungsimpulse formuliert und keine Empfehlungen ausgesprochen werden. Die in diesem Unterkapitel vorgestellten Handlungsimpulse sind nicht zwangsläufig für die Pfadentwicklung einer jeden Hochschule relevant, sie sind auch nicht vollständig, da jenseits der hier skizzierten Pfade auch andere Wege möglich erscheinen. Auf der Basis der $\mathrm{Zu}$ sammenführung und der Interpretation der Ergebnisse der Inhaltsanalysen sind es aber die beiden Handlungsfelder, welche als wesentlich abgeleitet wurden (s. Abbildung 34). 


\subsubsection{Eigenraum strategisch gestalten}

Hochschulen sind strategiefähig! In den vergangenen Jahren wurde die Autonomie der Hochschulen erweitert; auf diese Weise entstand ein von Hochschulen strategisch zu gestaltender Raum, der in diesem Abschnitt als Eigenraum charakterisiert wird - mithin als der Raum, welcher im Rahmen der Hochschulautonomie von hochschulischen Akteur*innen erobert und relativ unabhängig von Umweltweinflüssen gestaltet werden kann. Jedoch - und dieser Einwand hat Gewicht folgen Hochschulen weniger zweckrationalen Verfahren als vielmehr einem situativ-opportunistischen Muddling-Through, wie es von Entscheidungstheoretikern (Cohen et al., 1972) im Garbage-Can-Modell, das explizit Hochschulen zum Gegenstand hat, skizziert wurde. Dort wird die Idee direkter Zweck-Mittel-Relationen verabschiedet, Handlungen können Lösungen, aber auch Probleme darstellen, und was eine Lösung oder ein Problem darstellt, ist letzthin abhängig von Kontext und Sichtweise. Der strategisch gestaltbare Raum löst sich also kaum erobert - schon wieder auf.

Promotoren ${ }^{8}$ identifizieren: Hilfreich kann es daher sein, auf einen Ansatz des Innovationsmanagements zurückzugreifen, welcher das Problem der Innovation als ein Zusammenspiel verschiedener Rollen begreift: Das Promotorenmodell (Hauschildt, 1997) begreift das Innovationsgeschehen als arbeitsteiligen Zusammenhang, in welchem verschiedene Rollen zusammenwirken müssen, um innerhalb der Organisation Innovationen zu realisieren. Diese Rollen tragen dazu bei, verschiedene Barrieren zu überwinden: Der Fachpromotor vermag aufgrund seiner Expertise die Hürde des Nicht-Wissens zu meistern, der Machtpromotor hingegen trägt dazu bei, die organisationale Trägheit - also die Hürde des Nicht-Wollens - zu überwinden. Der Prozesspromotor schließlich trägt Sorge, dass in den täglichen Routinen die Hürde des Nicht-Dürfens nicht zum Blockadeinstrument wird.

Promotoren - und das ist das Problem der organisationalen Anarchie der Hochschulen - sind schwieriger zu identifizieren, als dies in herkömmlichen Organisationen der Fall ist. Macht ist in Hochschulen

8 Promotoren und Opponenten werden nicht gegendert, da es sich um feststehende Begriffe innerhalb des Promotorenmodells handeln (Hauschildt 1997). 
etwa weniger an hierarchische Positionen gebunden. Daher ist die Rolle des Machtpromotors eine, welche - insbesondere bei weitreichenden Innovationen - oftmals erst aufwändig zusammengefügt werden muss. Dazu passt, dass die befragten Hochschulleitungen ihre eigene Rolle nicht immer als die eines machtvollen Akteurs begreifen. Im Gegenteil: Mächtig sind immer die anderen: das Ministerium, welches mit Verordnungen agieren kann und Ressourcen kontrolliert, die Hochschulgremien, welche Entscheidungen blockieren oder unterlaufen können, die Fakultäten, welche eigene Politiken verfolgen und eine Machtakkumulation der Hochschulleitungen regelmäßig beargwöhnen. Aus dem Blick gerät dabei allzu leicht, dass sich gerade Hochschulleitungen oftmals in einer rechtlich unsicheren Situation agierend - in der Corona-Krise als außerordentlich handlungsmächtig erwiesen haben. Sie können und das ist ein Befund, der sich aus der Corona-Krise ergibt, durchaus die Rolle eines Machtpromotors ausüben. Allerdings gelingt dies desto besser, je stärker sie in der Lage sind, Machtkoalitionen zu bilden. Die Rolle des Machtpromotors in Hochschulen darf daher nicht unterkomplex verstanden werden. Eine Machtkoalition bedarf eines strategisch geschickten Agierens in den Gremien. Nicht umsonst sind gute Hochschulmanager*innen oftmals auch Gremienvirtuosen, welche die Gremien als politische Arenen zu nutzen vermögen. Und - auch dies ist zu von Bedeutung - Machtpromotoren agieren auf einer formalen Ebene, aber auch auf einer informalen, jenseits der organisationalen Strukturen, mitunter intransparent, lichtscheu, aber darum umso wirkmächtiger.

Geht man davon aus, dass Hochschulen sich in der Zeit nach der Corona-Krise neu positionieren und darüber entscheiden müssen, welche Elemente digitalen Lehrens und Lernens in den hochschulischen Alltag integriert werden sollen, also eine je eigene Pfadentwicklung anstreben werden, so erweist sich der Umstand, dass Macht in Hochschulen verteilt und wenig zentralisiert ausgeübt wird, möglicherweise eher als ein Vorteil denn als Nachteil. Die Bildung strategischer Koalitionen nötigt die Akteur*innen dazu, Diskurse zu führen, eigene Positionen zu begründen und vielfältige Interessen einzubeziehen. Allerdings bedarf es dann der Schaffung geeigneter Diskursarenen sowie der Geduld und der Zeit, um diese Prozesse tatsächlich bis zur Pfadentwicklung fortzuschreiben. 
Fachpromotoren, also diejenigen, welche in den strategischen Gestaltungsprozess das notwendige Wissen einbringen, sind ebenfalls oftmals nicht dort zu finden, wo man sie suchen möchte, sondern in der Hochschule mitunter so verborgen, dass sie schwer in die Innovationsprozesse einzubeziehen sind. Erfahrungen mit Digitalisierung der Lehre waren in den Hochschulen bei einzelnen Akteur ${ }^{\star}$ innen, die sich zum Beispiel in Weiterbildungsformaten ein Übungsfeld geschaffen hatten, durchaus vorhanden. Es gilt also, innovative Lehrende, die Lehrbeispiele entwickelt haben und diese zu teilen bereit sind, für die Rolle des Fachpromotors zu gewinnen und damit die Diffusion der Innovation zu gewährleisten. Fachpromotoren können auch Studierende sein, denn sie sind die Expert*innen für gelingendes Lernen. Das bedeutet jedoch, dass diese Akteur*innen, vormals eher randständig und nicht im Kernbereich der Lehre verortet, nun in eine zentrale Position gebracht werden und damit das arbeitsteilige Gefüge der Hochschulen in Bewegung, möglicherweise auch in Unordnung versetzt wird. Das ist insbesondere dann der Fall, wenn die eigentlichen Inhaber der Rolle des Fachpromotors - also die regelmäßig dafür zuständigen organisationalen Bereiche - in organisationalem Konservatismus gefangen sind und diese neue Unordnung nicht als Chance für die Hochschule, sondern vielmehr als Gefährdung mühsam ausbalancierter Ordnung empfinden.

Schließlich geht es darum, die Innovationsprozesse zu beschützen und Sorge zu tragen, dass es den Hochschulen nicht an Kraft gebricht, die Prozesse bis zu einem bestimmten Reifegrad zu führen. An dieser Stelle kommt der Rolle des Prozesspromotors eine nicht zu unterschätzende Bedeutung zu. Da sowohl die Akteur*innen, welche die Rollen des Macht- als auch des Fachpromotors innehaben, oftmals sehr vielfältigen Anforderungen unterliegen, laufen sie Gefahr, den Prozess nicht so nachhaltig begleiten zu können, wie es angemessen wäre. Daher sollte ein Prozesspromotor benannt werden, der den Innovationsprozess begleitet und den Prozessfortschritt sichert.

Barrieren identifizieren: Um den Prozess der Transformation so zu gestalten, dass er zielgerichtet und zukunftsorientiert abläuft, ist Identifikation und Zusammenspiel von Promotoren eine notwendige Bedingung. Darüber hinaus ist von Bedeutung, dass den Akteur*innen, die sich in diese Rollen begeben und so den Innovationsprozess fördern, 
mit Achtung begegnet wird. Promotoren sind in Innovationsprozessen regelmäßig mit Störungen konfrontiert - die auch daraus resultieren, dass Innovationen behindert, verzögert oder in Gänze sabotiert werden sollen. Daher gilt es sowohl strukturelle Barrieren zu überwinden als auch Blockaden, welche von Akteur*innen, die in Verhinderungskoalitionen agieren, errichtet wurden, zu umgehen oder diese zu vermeiden. So haben Promotoren zu vergegenwärtigen, dass sich in den Hochschulen der Unwille dahingehend artikuliert, dass (weiterhin) Ressourcen für die Digitalisierung oder die gemeinsame Entwicklung von zukünftigen Lehr-/Lernformaten gebunden werden. Damit wird grundsätzlich die Pfadentwicklung in Frage gestellt und Opponenten werden danach trachten, einzelne Realisierungsschritte zu verhindern. Das Ensemble der Promotoren hat dann die Aufgabe, dem entgegenzuwirken. Hier können die im Rahmen des Gabage-Can-Modells formulierten Handlungsempfehlungen geeignete Gedankenimpulse liefern (Cohen \& March, 1974). Um Barrieren abzubauen, muss viel Zeit investiert werden, um Opponenten ggf. auch auszusitzen. Bei allen Bemühungen wird es nämlich nicht möglich sein, alle Opponenten zu Promotoren zu lassen. Hier braucht es viel Zeit und Geduld, um sich von einzelnen Fehlschlägen nicht entmutigen zu lassen. Dabei kann es auch hilfreich sein Status gegen Substanz tauschen zu tauschen: d.h. symbolisch den Gegner ${ }^{\star}$ innen das Feld überlassen, substanziell jedoch den Prozess zu kontrollieren. Wichtig ist es aber auch, dass Opponenten nicht ausgeschlossen, sondern in den Prozess eingebunden werden. So kann einerseits bei der einen oder anderen Person ein Umdenken erreicht werden, andererseits werden so Verhinderungskoalitionen aufgebrochen. Durch unauffälliges Steuern in kleinen, aber wirkungsvollen Schritten kann es Promotoren so möglich sein, das komplexe System in eine gewünschte Richtung zu lenken und schließlich die Pfadentwicklung zu interpretieren.

Soll die Pfadentwicklung nach der Corona-Krise fortgesetzt werden, so dürfte eine Herausforderung darin bestehen, die vorhandenen emergenten Strategieelemente zu bündeln und in stärkerem Maße, als dies bisher möglich war, eine geplante Strategie zu formulieren. Eine solche Strategie - das zeigt bereits die Vielfalt der emergenten Pfade wird zunächst von jeder Hochschule individuell formuliert werden müssen: Typisierte generalistische Strategien, wie sie aus einschlägigen 
Lehrbüchern bekannt sind, werden den Problemlagen nicht gerecht. Es mag sein, dass sich im Entwicklungsprozess standardisierbare Strategiemuster herausbilden werden, einstweilen sind diese jedoch nicht zu identifizieren. Dies hat zur Folge, dass sich für Hochschulen ein strategischer Entwicklungsraum öffnet und damit eine neue Unübersichtlichkeit entstehen dürfte, welche der einzelnen Hochschule Positionierungsmöglichkeiten verschafft.

Einen strategischen Kern bilden: Nimmt man die Handlungsmaxime von Cohen und March (1974), Beteiligungsmöglichkeiten zu schaffen, ernst, so gilt es, Partizipation zu organisieren. Dazu kann beispielsweise innerhalb der Hochschule ein strategischer Kern gebildet werden, in welchem die Promotoren versammelt sind. In diesen Kern sollten aber auch die Akteur*innen integriert werden, welche die Umsetzung der Strategie befördern oder behindern können. Die Partizipationsmöglichkeit trägt dann dazu bei, dass Vorbehalte und Barrieren abgebaut werden. Von Bedeutung ist darüber hinaus, hochschulische Strukturen und Gremien in die Pfadentwicklung einzubeziehen und nicht den Anschein zu erwecken, als sollten diese umgangen oder gar ausgehebelt werden. In den wesentlichen Gremien sind Interessenvertreter ${ }^{*}$ innen aller Hochschulgruppen vertreten und geben damit der Hochschule insgesamt eine Stimme. Die wesentliche Aufgabe des strategischen Kerns ist es dann nicht nur, ein gemeinsames Zielbild zu entwickeln, sondern auch Schritte zur Umsetzung der Strategie einzuleiten. Der strategische Kern sollte die Hochschule über den Prozess der Transformation hinweg begleiten.

Mögliche Pfade erschließen: Schließlich bedeutet die strategische Positionierung nach der Corona-Pandemie, dass Pfade im Kontingenzraum erschlossen werden müssen. Um dieses zu tun, gilt es, sich den eröffneten Kontingenzraum bewusst $\mathrm{zu}$ machen und ihn als das $\mathrm{zu}$ interpretieren, was er ist: nämlich ein Gestaltungsraum, in welchem sich Hochschulen einer Vielzahl von Möglichkeiten gegenübersehen. Hochschulen sind mit der Herausforderung konfrontiert, aus der Vielfalt der Möglichkeiten diejenigen zu wählen und zu kombinieren, welche eine konsistente Strategieformulierung ermöglichen. Dabei gibt es nicht den einen Pfad post Corona, sondern mehrere Wege, sich nach dem Pandemiegeschehen hinsichtlich der Digitalisierung der Lehre $\mathrm{zu}$ positionieren. Für Hochschulen ist es dann notwendig, die mögli- 
chen Pfade wahrzunehmen und davon ausgehend denjenigen Pfad $\mathrm{zu}$ identifizieren, den sie einschlagen wollen. Ob diese strategische Justierung inkrementell oder disruptiv erfolgt, hängt von der Standortbestimmung der jeweiligen Hochschulen ab.

\subsubsection{Hochschulumwelt gemeinsam gestalten}

Ein Begriff, der im Feld der Hochschulen in den letzten Jahrzehnten verstärkt Einzug gehalten hat, lautet: Wettbewerb (s. Kapitel 7.6). Neben der Frage, wie Hochschulen zukünftig ihren Eigenraum gestalten, wird damit vor allem die Frage adressiert, wie sich Hochschulen in der Bildungslandschaft positionieren. Auch wenn politisch sowie von den einschlägigen Verbänden einiges dafür getan wird, den Wettbewerb durch Clusterung von Hochschultypen einzuhegen und den „binary divide" (Müller-Böling, 2005)- also die Unterteilung in Universitäten und in HAWs - aufrechtzuerhalten, so ist doch zu konstatieren, dass Bewegung innerhalb des Feldes der Hochschulen zu verzeichnen ist. Für HAWs scheint hier das Moment der gemeinsamen Strategieentwicklung wesentlich zu sein, um nicht zwischen anderen Hochschularten zerrieben zu werden.

Eigene Positionierung ermöglichen: Die Frage, die sich stellt, lautet, wie sich Hochschulen im Bildungsraum positionieren. Zum einen verfolgen HAWs die Strategie eines academic drift, also der teilweisen Annäherung an die universitären Hochschulen. Zum anderen jedoch reklamieren sie eine andersartige Profilierung für sich, welche etwa den stärkeren Praxisbezug oder die Anwendungs- und Transferorientierung betont. Somit spielen HAWs ein doppeltes Spiel. Das erstere ist auf einer vertikalen Achse zu verorten und die HAWs versuchen dort, den Abstand zu den Universitäten zu verringern. Das zweite Spiel findet auf einer horizontal positionierten Achse statt und dort versuchen die einzelnen HAWs, einen Raum mittels andersartiger Positionierung für sich zu reklamieren. In der Summe führt das dazu, dass die binäre Differenzierung des Hochschulsystems zunehmend instabil erscheint. Digitalisierung schließlich eröffnet Hochschulen eine weitere Option der Positionierung innerhalb des Feldes der Hochschulen. Während bislang der Standort einer Hochschule Einzugsgebiete 
und Wirkungskreise der Hochschule zwar nicht vollständig, aber doch tendenziell definiert und nur wenige Hochschulen diese Begrenzungen zu durchbrechen vermögen, können mittels Digitalisierung nun auch andere Räume erschlossen werden. Hinzu kommt, dass Digitalisierung das Studium auch zeitlich durch den Einsatz asynchroner Lehr-/ Lernformate zu entgrenzen vermag. Wie stark diese Möglichkeiten der Differenzierung von Hochschulen und Studierenden in Anspruch genommen werden, hängt ganz wesentlich davon ab, wie intelligent diese Kombinationen aus traditioneller Hochschule und digitaler Erweiterung gestaltet werden. In diesem Sinne werden Hochschulen in Bälde die Rolle eines „institutional entrepreneurs“ (DiMaggio 1988) einnehmen können, der neue Lehr-/Lernformen in ein institutionalisiertes Setting überführt. Und damit verändert sich die Position der Hochschulen: Sie gerieren sich stärker als Gestalter ihrer Umwelt und weniger ausgeprägt als Anpasser an exogen bestimmte Umweltbedingungen.

Innerhalb des Kontingenzraums eröffnen sich also mehrere Dimensionen, welche Hochschulen zur Positionierung nutzen können. Das kann die räumliche und zeitliche Entgrenzung, die Formatvarianz, die Hybridisierung, die kooperative Entgrenzung und noch weitere sein. Aufgabe der Hochschulen ist es, diese Stellschrauben entsprechend ihrer Strategie und ihres Profils einzustellen und sich damit eindeutig zu positionieren.

Autonomiegrade nutzen: In der Beziehung zum Verordnungsgeber (Landesregierung bzw. einschlägiges Ministerium) hat sich in der Pandemiesituation ein besonderes Spannungsverhältnis offenbart. Einerseits sehnten sich die Hochschulen nach mehr Verbindlichkeit und Sicherheit, insbesondere dann, wenn Regelungstatbestände umgesetzt werden mussten. Andererseits war auch festzustellen, dass die sehr unbestimmte Situation den Hochschulen auch Freiheiten erschlossen hat, die von den Hochschulleitungen unterschiedlich interpretiert wurden. Einerseits löst eine Krisensituation naturgemäß ein Sicherheitsbedürfnis aus und führt dazu, dass genauere Vorgaben eingefordert werden. Andererseits jedoch schätzen Hochschulleitungen den Zugewinn der Autonomie als wertvoll, da dieser erlaubt, eine je spezifische Pfadentwicklung zu verfolgen. 
Begreift man den Wandel der Hochschulen als einen transformativen Prozess, geht es bei der Frage nach den Rahmenbedingungen um den Aufbau von Systemwissen. Um eine Vorstellung über einen wünschenswerten Zielzustand (Zielwissen) zu generieren, ist ein Grundverständnis über das funktionierende System unabdingbar. Es steht zu erwarten, dass Digitalisierung von Lehren und Lernen eine Weiterentwicklung des hochschulischen Feldes auslöst und diese Weiterentwicklung von verschiedenen Akteur*innen beeinflusst werden kann. In der neuen Unübersichtlichkeit ist die Position der Spieler einstweilen noch nicht festgelegt: Das jedoch bedeutet, dass eine zukünftige Strategie der Hochschulen nicht von bekannten und wohldefinierten Rahmenbedingungen her gestaltet werden kann, sondern dass die Strategie vorgibt, welche Rahmenbedingungen zukünftig notwendig sind. Durch die Pandemie ist hier ein Entwicklungsprozess angestoßen worden, der Möglichkeitsräume für Hochschulen eröffnet, die es zu identifizieren und zu gestalten gilt.

Neue Studienmodelle entwickeln: Eine Möglichkeit zur Gestaltung des Autonomieraums stellen neue Studienmodelle dar. In der Pandemie hat sich gezeigt, dass Hochschulen in einem Fernstudienmodell arbeiten können. Nun wurde schon mehrfach deutlich gemacht, dass die HAWs (und vermutlich auch andere Hochschularten) ihren Status als Präsenzhochschule nicht aufgeben werden. Dies hat viele gute Gründe, auf die schon vielfach eingegangen wurde. Dennoch lässt sich aus den vergangenen Semestern zumindest die Erkenntnis formulieren, dass neben den in der Vergangenheit etablierten Formaten andere, auch neue Formate zur Verfügung stehen, die zum Teil noch der Formung bedürfen. Mithilfe eines digitalen Konzepts ist es denkbar, berufsbegleitende Studienmodelle im regulären Studienbetrieb umzusetzen. Solche Modelle sind bisher fast ausschließlich im Bereich der wissenschaftlichen Weiterbildung zu finden, in deren Rahmen Studiengänge nicht kapazitätswirksam sind. Auch wenn berufsbegleitende Studiengänge nicht automatisch weiterbildend - im Sinne von an die Berufspraxis anschließend - sind, kann es hier doch im positiven Sinne zu einer stärkeren Entgrenzung zwischen Weiterbildung und regulärem Studium kommen. Im Idealfall führt dies zu einer weiteren Öffnung von Übergängen und zu mehr Partizipation an hochschulischer Bildung insgesamt, weil so auch Personen ein Studium ermöglicht 
wird, welche berufstätig sind, sich aber ein weiterbildendes, gebührenpflichtiges Studium nicht leisten können, bzw. welche ein Studium anstreben, das nicht direkt an ihre jetzige Berufstätigkeit anschließt. Hier werden differenzierte Gestaltungen von Bildungsbiografien möglich.

Kooperationen eingehen: Die genannten Impulse können durch einen Blick in die Peripherie ergänzt werden. Es stellt sich hier die Frage nach strategischen Kooperationen über die Hochschulgrenzen hinaus. Bei der Auslotung von neuen Autonomiegraden dürfte sich auch die Ausprägung der hochschulischen, interorganisationalen Beziehungen als bedeutsam erweisen. Digitalisierung ermöglicht die Aufnahme neuer Kooperationsbeziehungen und senkt - ein nicht zu unterschätzender Vorteil - die Transaktionskosten, also - grob vereinfacht formuliert - die Organisationskosten der Kooperation. Damit ist ein zweiter Gesichtspunkt der Umweltgestaltung benannt: Die Organisationsgrenzen werden durchlässiger. Es wird von Bedeutung sein, ob die HAWs ein geteiltes Verständnis von Autonomie entwickeln können, da dies eine Bedingung darstellt, um die dem Hochschultypus eigenen Handlungsoptionen auch zu ergreifen.

Gerade um das Alleinstellungsmerkmal der HAWs herauszuarbeiten, wird vielfach genannt, dass man gemeinsam definieren muss, wie die Zukunftsperspektive aussieht. Viele der genannten Punkte der Profilschärfung könnten auch den Schluss nahelegen, dass nun jede Hochschule für sich möglichst gut ausloten muss, wo in dem Kontinuum zwischen Präsenz und Digitalem sie zukünftig steht und wie sie ihren jeweiligen Standort gestaltet. Gerade darin liegt aber eine Gefahr, die nicht nur Einzelne betrifft, die durch eine schwache Transformationsstrategie abgehängt werden können. Die Hochschulen für Angewandte Wissenschaften müssen sich insgesamt fragen, was sie denn zukünftig auszeichnen soll.

Studierende fordern ein zeitgemäßes Studium: „Wozu braucht die Welt eine Hochschule, wenn alle Vorlesungen online verfügbar sind?” (Scharmer, 2019) Diese Frage müssen alle Hochschulen beantworten und es liegt eine Stärke darin, dies gemeinsam zu tun. So entsteht kein Verdrängungswettbewerb, sondern man begreift die pandemiegetriebenen Veränderungen als gemeinsame Gestaltungsaufgabe. Die hier vorgestellte Studie hat einen hochschulübergreifenden Ansatz 
gewählt. Dabei konnte festgestellt werden, dass alle Hochschulen ähnliche Chancen und Risiken identifizieren und daran gehen, diesen durch Strategieentwicklung zu begegnen. Hochschulen werden sich teilweise neu erfinden müssen. Die eingangs zitierte Frage Adornos hat in diesem Kapitel eine vorläufige Antwort erhalten; gleichwohl gilt die Erkenntnis von Peter Høegs (1994) Romanheldin Smilla, die weiß: „Das Schwere ist das Neue“ (S. 355). 


\section{Ausblick}

Welche Entwicklungspfade für die Hochschullehre post Corona können aus den Erfahrungen der digitalen Semester abgeleitet werden? Seit dem Sommersemester 2020 wurden Hochschulen in eine völlig neue Situation geworfen, die sie durch hohes Engagement aller Akteur*innen - Studierender, Lehrender und Leitungen - bewältigen konnten. Viele Aspekte und Herausforderungen dieser Situation werden mit Wegfall der Corona-Auflagen obsolet, weil sie ganz konkret an die Restriktion gebunden sind, dass Hochschule nur digital stattfinden darf. Wenn ein Präsenzmodus wieder möglich wird, müssen sich Hochschulen dennoch die Frage stellen, welche Chancen sie in der Krisensituation erkannt haben und wie sie diese in eine strategische Pfadentwicklung überführen. In diesem Sinne werden sich Hochschulen und damit die hochschulische Bildungslandschaft in einen Veränderungsprozess begeben müssen. Hochschulen haben bereits in der Corona-Situation erste Strategien entworfen, um den neuen Möglichkeitsraum zu gestalten.

Für die hochschulische Lehre wurden Vorteile des Digitalen erfasst bzw. erschlossen. Es wurden aber auch Grenzen aufgezeigt, welche die Chancen der Präsenz wieder stärker in den Vordergrund stellen. Grundsätzlich erarbeiten Hochschulen während der Pandemie mit der digitalen Lehre kein Neuland. Im einschlägigen Forschungskontext sind die Chancen und Herausforderungen hinreichend diskutiert. In der Befragung bestätigten sich viele der in der Literatur beschriebenen Aspekte, es zeigte sich aber auch, dass Hochschulen, die bislang im Wesentlichen als Präsenzhochschulen arbeiteten, erneut vor den bereits validierten Herausforderungen stehen bzw. neue Chancen im digitalen Setting erkennen. Das Neue ist nicht, dass es nach Corona digitale Lehre gibt, sondern es zeigt sich in der Art und Weise, wie Präsenzhochschulen post Corona digitale Lehre in ihre Strategie integrieren. Basierend auf einem neuen, gemeinsamen Verständnis von zukünftigem Lehren und Lernen ist es nun geboten, den Mix der 
Lehr-/Lernformate neu zu definieren. Dies hängt unmittelbar mit der Frage nach der zukünftigen Strategie der Hochschulen zusammen.

Grundsätzlich hat es Hochschulen geholfen, den Möglichkeitsraum in der unsicheren Situation durch ein Zusammenspiel von Handlung und Strukturbildung zu erschließen und erste emergente Handlungsstrategien zu entwerfen. Auf diese Weise ließ sich das Kompetenzgap, das zu Beginn der Pandemiesituation auftrat, in großen Teilen schließen und es wurden neue Optionen für Optimierungsprozesse identifiziert. Vor allem hinsichtlich ihrer Begegnungs- und Kommunikationsstrukturen waren Hochschulen besonders gefordert. Die Entgrenzung verschiedener Lebensbereiche führte zudem zu hoher Belastung. Das Miteinander wurde neu definiert. Diese Gestaltung von Lehr-, Lern- und Arbeitswelt wird auch zukünftig Raum einnehmen.

Im Zuge der Pandemie haben Hochschulen neue, innovative Bereiche bzw. Innovationsfelder für die Zukunft identifiziert. Die Digitalisierung an Hochschulen tritt als Wettbewerbsfaktor zutage, was dazu führt, dass sich Hochschulen hinsichtlich einer zukunftsfähigen Strategie neu aufstellen müssen. Die Identifikation von Promotoren im Hochschul- und Bildungssystem sowie die Entwicklung tragfähiger Kooperationen können dabei entscheidende Faktoren sein.

Hochschulen befinden sich bereits jetzt in einem Veränderungsprozess. Diese Veränderung wurde durch die Pandemie getriggert, wird aber mit dem Verschwinden der Corona-Auflagen nicht mehr wegzudenken sein. Für Hochschulen stellt die Pandemie eine Krise dar. Die Resilienzfähigkeit der Hochschulen wird sich nicht nur in der Überwindung dieser Krise zeigen, sondern auch dadurch wie und ob Hochschulen gestärkt aus der Krise hervorgehen und ob es ihnen gelingt, die schon angelegte Veränderung in eine strategische und gesteuerte Pfadentwicklung zu überführen. Nur so können Hochschulen aus einem reaktiven Verhalten hinsichtlich der von außen erzwungenen Bedingungen der Corona-Pandemie in eine aktive und planende Handlungsrolle gelangen. In diesem Sinne befinden sich die Hochschulen in einem transformativen Prozess, in dem sie den Kontingenzraum definieren und gestalten können. Hier kommt es darauf an, dass hochschulische Akteur*innen den Spielraum innerhalb der Kontingenz nicht nur als Vakuum hinsichtlich fehlender Struktur und 
Rahmenbedingungen verstehen, sondern dass sie hier eine Chance wahrnehmen, ihre Autonomiegrade neu zu nutzen und selbst zu definieren. Diesen Prozess, der sich dadurch auszeichnet, dass zunächst neues Wissen darüber aufgebaut werden muss, wie der zukünftige Zielzustand aussehen soll, kann durchaus als Transformation beschreiben werden. Immer wieder wird beobachtet, dass Akteur ${ }^{*}$ innen partizipativ und auf Augenhöhe eingebunden werden und dass dadurch Entscheidungsprozesse Transparenz erlangen. Durch die experimentelle Situation wurden zudem viele Prozesse automatisch iterativ. Im Rahmen dieser Iteration wurden die einzelnen Prozessschritte immer wieder reflektiert. Mögliche Gelingensbedingungen für den Umgang mit der Pandemiesituation sowie Handlungsstrategien wurden ebenfalls reflektiert und diskutiert.

Die grundsätzliche Herausforderung liegt nun darin, dass in der Pandemie zutage getretene Defizite, aber auch Chancen und Möglichkeiten, in eine strategische Pfadentwicklung überführt werden. Denn auch ohne Corona-Auflagen können die Lessons Learned aus dem Pandemiegeschehen für die Gestaltung von Hochschulen in der $\mathrm{Zu}$ kunft wichtige Implikationen liefern. Die wesentlichste Frage ist, welche Rolle digitale Lehre zukünftig spielen kann und soll. Dabei ist eine zentrale Prämisse: HAWs sind Präsenzhochschulen und zeichnen sich im Besonderen durch die niederschwellige Studierende-LehrendeInteraktion ebenso wie durch den Praxisbezug und die Förderung praxisnaher Fähigkeiten aus. Dieses profilbildende Merkmal wird auch in der Zukunft ein wichtiges Element im „neuen, hybriden Hochschulkonzept" darstellen und es lässt sich auch auf andere Hochschularten übertragen. Die Gewichtung der Präsenz wird durch die Relevanz der Hochschulen als Ort der Persönlichkeitsbildung und als Sozialisationsraum zusätzlich unterstrichen.

Andererseits haben die digitalen Elemente deutliche Vorteile hervorgebracht, die sich beispielsweise in der Flexibilisierung von Lehren und Lernen zeigen. Durch die Durchmischung von Präsenz- und digitalen Formaten wird der methodisch-didaktische Blumenstrauß größer. Wenn sich Hochschulen hier auf ein grundsätzliches, gemeinsames Konzept einigen, liegen neue Freiheitsgrade in der Lehre darin, diese jeweils individualisiert und innovativ auszugestalten. Hier ist es eine 
Chance, die schon vielfach in der Literatur beschriebenen Möglichkeiten der digitalen Lehre aufzugreifen und neu zu kontextualisieren.

Mit der hier vorliegenden Studie gelang es, unter Zuhilfenahme des qualitativen Ansatzes viele Aspekte der Geschichten zu beleuchten, die sich in Hochschulen in den letzten beiden Semestern unter CoronaBedingungen ereigneten. Es konnten gezielte Einblicke in die Gestaltungsvorgänge innerhalb des Kontingenzraums erhalten werden. Dabei war es ein besonderes Anliegen, das Subjekt in den Fokus der Betrachtung zu rücken. Eine Verbindung der verschiedenen Perspektiven zwischen Studierenden, Lehrenden und Hochschulleitungen sollte für einen ganzheitlichen Blick auf das Hochschulgeschehen sorgen. Ausgehend von der hier vorliegenden Studie gibt es weiteren Forschungsbedarf hinsichtlich der Beobachtung der Entwicklung an Hochschulen im zeitlichen Verlauf. Besonders nach Wegfall der Corona-Auflagen sollte untersucht werden, inwiefern Hochschulen post Corona in der Lage sind, den Digitalisierungsschub in eine strategische Pfadentwicklung zu überführen und damit die Restauration zu überwinden und in einen transformativen Prozess umzusetzen. 


\section{Anhang}

\section{Anhang 1: Interviewleitfaden Hochschulleitungen}

\section{Leitfaden Zugang 1: Hochschulleitungen (Verwaltung - Lehre - Studium)}

\begin{tabular}{ll}
\hline Themen/Fragen & Wie war Ihre Hochschule vor Corona (Februar 2020) aufgestellt? \\
\hline Voraussetzungen & (o) allgemein \\
(o) hinsichtlich Digitalisierung in der Verwaltung \\
(o) hinsichtlich Digitalisierung bei Lehrenden/Studierenden \\
Wie waren die Rahmenbedingungen? \\
(o) Rahmenbedingungen in der Verwaltung? \\
(o) Rahmenbedingungen in der Lehre / im Studium?
\end{tabular}

Handlungen/ Maßnah-
men \& Entscheidungs-
prozesse

Können Sie erst mal berichten, wie es in Ihrer HAW gelungen ist, die beiden digitalen Semester durchzuführen?

Was waren die Reaktionen, als Sie von der Aussetzung des physischen Präsenzsemesters der Hochschulen erfahren haben?

(o) Welche Maßnahmen wurden seitens der HSL eingeleitet?

(o) Inwiefern gab es ein Konzept zur Bewältigung der Pandemiesituation?

Wer waren die wichtigsten Akteure bzw. Akteur*innengruppen?

(o) Inwiefern gab es noch weitere Maßnahmen?

(o) Welche Hilfestellungen gab es in der Verwaltung / für Lehrende?

(o) Inwiefern wurden Lehrende in den Change-Prozess einbezogen?

(o) Welche Unterstützung gab es für Studierende/Erstsemester?

Inwiefern hat sich die Kommunikation in der Hochschule verändert?

(o) Wie lief der gegenseitige Austausch?

(o) Inwiefern hat sich die Kommunikation in den Gremien verändert?

Inwiefern hat sich die Strategie der HS verändert durch Corona?

Welche Entscheidungen mussten Sie als HSL treffen?

(o) Wie wurde mit der Tragweite von Entscheidungen umgegangen?

(o) Wurden andere Führungsebenen in der HS eingebunden?

(o) Wie wurden wichtige Entscheidungen in die HS kommuniziert?

(o) Wie sah der Informationsfluss vonseiten des Ministeriums aus?

(o) Gab es Support von der MWK? Hochschulrektorenkonferenz?

Wie lief es in der Verwaltung bezüglich Prüfungsgremien?

Wie ist Ihre persönliche Einschätzung zur Lehrqualität in Ihrer Hochschule in den Corona-Semestern?

Inwiefern gab es eine Veränderung in der Lehre, außer, dass sie statt in physischer Präsenz digital stattfindet?

(o) didaktische Veränderung, soziale Interaktion, Change-Management 


\begin{tabular}{ll}
\hline Herausforderungen & Wenn Sie jetzt rückblickend auf die zwei Semester schauen, wo sehen \\
Sie als HSL die größten Herausforderungen? \\
(o) Wo liegen die aktuellen Herausforderungen für Sie als HSL? \\
(o) Inwiefern konnten alle Beteiligten im digitalen Semester mitgenom- \\
men werden? Was bedeutete das für Sie als HSL? \\
(o) Was waren die größten Herausforderungen in der Verwaltung? \\
(o) Was waren die größten Herausforderungen in der Lehre? \\
(o) Worin wird Ihr Hauptaugenmerk als HSL hinsichtlich der zukünfti- \\
gen Gestaltung der Lehre liegen? \\
(o) Was waren die größten Herausforderungen für die Studierenden? \\
\hline Gefahren & Wo sehen Sie als HSL vor dem Hintergrund der letzten zwei Semester \\
& Gefahren für die Hochschule? \\
\hline Chancen & Wo sehen Sie als HSL vor dem Hintergrund der letzten zwei Semester \\
& Chancen für die Hochschule? \\
\hline Anforderungen & Welche Anforderungen sehen Sie als HSL zukünftig für die Hochschu- \\
& len? \\
& Was bedarf es als HSL seitens der Politik / des zuständigen Ministeri- \\
& ums? \\
& Inwiefern sehen Sie als HSL spezifische Themen für HAWs in Abgren- \\
& zung zu den Unis? \\
\hline Was ist Ihr Standpunkt: Wie sieht die Hochschule - Zeitraum bis Ende \\
der Corona-Lehre - aus? \\
Wie sieht die Hochschule der Zukunft 2025 aus? \\
Haben wir im Laufe des Interviews Aspekte ausgelassen, die Sie sich \\
vielleicht schon im Vorfeld überlegt haben oder die Ihnen gerade noch \\
durch den Kopf gehen?
\end{tabular}

Anmerkungen $(o)=$ optionale Fragen, HSL $=$ Hochschulleitung. 


\section{Anhang 2: Interviewleitfaden Lehrende}

Leitfaden Zugang 2: Lehrende

\begin{tabular}{ll}
\hline Themen & Fragen \\
\hline Warm-up & Wie sieht jetzt in den Corona-Semestern Ihr Lehrort aus? Was ist das \\
& für ein Raum oder Platz, von dem aus Sie lehren? \\
\hline Lehre & Wie kamen Sie mit der Umstellung im SoSe 2020 auf die digitale Lehre \\
& zurecht? Und was hat da mitreingespielt? \\
& (o) Welche Herangehensweisen haben Sie entwickelt, um die digitale \\
& (o) Wehre erfolgreich zu gestalten? \\
& (o) Wie kamen Sie Ihre Lehre in den digitalen Semestern technischen Aspekten zurecht? \\
& (o) Wie sind Sie damit umgegangen, wenn die Studierenden Kamera \\
& (o) Was Ton ausgeschaltet hatten? \\
& (o) Was lief besser / Was lief schlechter? \\
& (o) Wie hat sich die Struktur ihrer Vorlesungen verändert?
\end{tabular}

Kommunikation und Interaktion
Wie haben Sie digitale Kommunikation und Interaktion, d. h. den Austausch mit den Studierenden in den Corona-Semestern organisiert?

(o) Wie haben Sie mit Ihren Studierenden den Kontakt gehalten?

(o) Worüber haben Sie mit Ihren Studierenden gesprochen?

(o) Wie haben Sie Gruppenarbeit/Austausch der Studierenden untereinander gefördert?

(o) Wo und wie finden nun im Digitalen die Gespräche, die früher am Kaffeeautomaten oder in der Mensa geführt wurden, mit den Studierenden statt?

Wie haben Sie den Austausch mit/unter Kolleg*innen organisiert?

(o) Worüber haben Sie sich mit Ihren Kolleg*innen und anderen Mitarbeitenden der Hochschule ausgetauscht?

(o) Was wertschätzen Sie am Austausch in den „Normal“-Semestern?

(o) Was haben Sie in den digitalen Semestern vermisst?

(o) Was lief bzgl. Kommunikation besser in den digitalen Semestern? anders organisieren müssen?

(o) Wie war es mit Pflege und Betreuung von Angehörigen?

(o) Haben Sie Corona-Ängste? Wenn ja, worauf beziehen diese sich?

(o) Wie ist Ihre Work-Life-Balance in den digitalen Semestern im Vergleich zu denen in Präsenz?

(o) Wodurch ersetzen Sie das reduzierte Leben außerhalb der Lehre?

Gesamtsituation

Was machen Sie aktiv, um mit der Gesamtsituation zurechtzukommen?

(o) Im Rückblick: Wie bewerten Sie die Gesamtsituation?

(o) Welche Strategien haben Sie entwickelt, um die Gesamtsituation zu organisieren?

Schluss

Inwiefern hat sich Ihr Blick auf die "reguläre" Lehre nach den zwei digitalen Corona-Semestern verändert?

(o) Wie hat sich Ihre didaktische Kompetenz verändert?

(o) Was würde Ihnen in den nächsten Semestern helfen? 
Welche Lehren/Fazits sollte Ihre Hochschule aus der Corona-Zeit ziehen?

(o) Was würden Sie aus den digitalen Semestern für die zukünftige Hochschule mit herübernehmen und was nicht?

(o) Welche Rolle spielen die Präsenz und das Campusleben vor Ort? Inwiefern wird sich das verändern?

(o) Inwiefern wird sich Ihre Rolle als Lehrende verändern?

\begin{tabular}{ll}
\hline Abschlussfrage & Was wünschen Sie sich für die Post-Corona-Zukunft hinsichtlich Lernens \\
und Lernen?
\end{tabular}

Anmerkungen $(o)=$ optionale Fragen. 


\section{Anhang 3: Interviewleitfaden Studierende}

Leitfaden Zugang 2: Studierende

\begin{tabular}{|c|c|}
\hline Themen & Fragen \\
\hline Warm-up & $\begin{array}{l}\text { Wie sah Ihr Lernort in den letzten beiden Semestern aus - wo und wie } \\
\text { haben Sie gelernt? }\end{array}$ \\
\hline Studium & $\begin{array}{l}\text { Wie kamen Sie mit dem digitalen Lernen im Vergleich zum Präsenz- } \\
\text { studium vor Corona zurecht? Was hat damit reingespielt? } \\
\text { (o) Womit kamen Sie gut zurecht? Was war für Sie schwierig? } \\
\text { (o) Wie haben Sie das Studium gestaltet? } \\
\text { (o) Wie haben Sie sich in den digitalen Semestern organisiert? } \\
\text { (o) Hat sich der Workload verändert im Vergleich zu „Normal“-Se- } \\
\text { mestern? } \\
\text { (o) Wie kamen Sie mit den technischen Aspekten zurecht? } \\
\text { Lehrende haben immer wieder berichtet, dass sie Mühe hatten, wirk- } \\
\text { lich in Kontakt zu den Studierenden in der digitalen Veranstaltungssi- } \\
\text { tuation zu kommen. Wie haben Sie das erlebt als Studierende? } \\
\text { (o) Haben Sie Kamera und Ton bei synchronen Veranstaltungen aus- } \\
\text { gemacht? Wenn ja, wieso? } \\
\text { (o) Welche Aspekte haben Sie motiviert, aktiv an der digitalen Lehre } \\
\text { teilzunehmen und zu lernen? } \\
\text { (o) An welcher Stelle hat sich das Lernen vereinfacht? } \\
\text { (o) An welcher Stelle ist es schwieriger geworden? } \\
\text { (o) Wie haben Sie außerhalb der synchronen Veranstaltungen das } \\
\text { Lernen organisiert? } \\
\text { (o) Was war es mit den Prüfungen? } \\
\text { (o) Welche Gedanken machen Sie sich über Ihren Studienverlauf und } \\
\text { Studienerfolg? }\end{array}$ \\
\hline $\begin{array}{l}\text { Kommunikation } \\
\text { und Interaktion }\end{array}$ & $\begin{array}{l}\text { Wie war die Umstellung auf eine rein digitale Kommunikation für Sie? } \\
\text { Wie ist es jetzt? } \\
\text { (o) Mit wem haben Sie sich ausgetauscht? } \\
\text { (o) Über was haben Sie sich mit Ihren Kommiliton*innen ausge- } \\
\text { tauscht? } \\
\text { (o) Wo und wie finden nun im Digitalen die Gespräche, die früher } \\
\text { am Kaffeeautomat oder in der Mensa geführt wurden, statt? } \\
\text { (o) Sind Sie im Vergleich zu „Normal“-Semestern eher zum zur Ein- } \\
\text { zelkämpfer*in geworden? } \\
\text { (o) Wie haben Sie in der Gruppe zusammengearbeitet? } \\
\text { (o) Was haben Sie mit Ihren Lehrenden und anderen Mitarbeitenden } \\
\text { der Hochschule besprochen? } \\
\text { (o) Wie sind Sie an nötige Informationen gekommen? } \\
\text { (o) Was wertschätzen Sie am Austausch in den „Normal“-Semestern? } \\
\text { Was haben Sie in den digitalen Semestern vermisst? Wie wichtig } \\
\text { sind „echte“ Menschen? }\end{array}$ \\
\hline
\end{tabular}

Private Situation

Wie hat sich Ihre Lebenssituation in den digitalen Semestern verändert?

(o) Jetzt gibt es die ganzen Partys, Studi-Events, Zusammentreffen am Abend, das Leben auf dem Campus nicht mehr. Was davon fehlt Ihnen am meisten? 
(o) Wie ist Ihre finanzielle Situation? Wie gehen Sie damit um?

(o) Wie kommen Sie mit Ihren Nebentätigkeiten zurecht? Was hat sich da verändert?

(o) Wohnort und Lernort sind ja nun in den digitalen Corona-Semestern identisch. Wie gehen Sie mit dieser neuen Situation um?

(o) Wie ist es mit Pflege und Betreuung von Angehörigen?

(o) Wie ist ihre Study-Life-Balance in den digitalen Semestern im Vergleich zu denen in Präsenz?

(o) Wodurch ersetzen Sie das reduzierte/veränderte Leben außerhalb des Studiums?

Gesamtsituation Was machen Sie aktiv, um mit der Gesamtsituation zurechtzukommen?

(o) Im Rückblick: Wie bewerten Sie die Gesamtsituation der digitalen Corona-Semester?

(o) Was läuft insgesamt besser als in den Präsenzsemestern?
Was würde Ihnen in den nächsten Corona-Semestern helfen?

Welche Lehre sollte Ihre Hochschule aus der Corona-Zeit ziehen?

(o) Welche Elemente aus den digitalen Semestern sollten in die Hochschule nach Corona übernommen werden?

Wenn Sie sich jetzt vorstellen, Sie würden sich jetzt zum ersten Mal an einer Hochschule bewerben, inwiefern würde der Anteil des digitalen Lernens Ihre Entscheidung beeinflussen?

Abschlussfrage Zum Abschluss würden wir gerne noch ein Statement zu Ihren Wünschen für die Post-Corona-Zukunft der HAWs hören.

Anmerkungen $(o)=$ optionale Fragen . 


\section{Anhang 4: Interviewleitfaden treibende Lehrende}

Leitfaden Zugang 3: Treibende Lehrende

\begin{tabular}{ll}
\hline Phasen & Fragen nach Themenbereichen \\
\hline Warm-up & Zurzeit laufen noch Ihre Veranstaltungen (Ihre Prüfungen) im digitalen \\
& Wintersemester. Wie ist denn da gerade Ihre Stimmung? \\
\hline Vorerfahrungen & Mediendidaktik: \\
vor Corona & Welche Lehr-/Lernformate haben Sie bisher genutzt? (digitale Anteile, \\
& Prüfungen, Team-Teaching) \\
& (o) Herausforderungen, Verluste, Möglichkeiten \\
& Technisches \& Kompetenz: \\
& Welche Programme und Plattformen haben Sie (wie) vor dem digitalen \\
& Semester schon für die Lehre genutzt? \\
& Kommunikation \& Interaktion: \\
& Wie haben Sie vor Corona mit den Studierenden kommuniziert/inter- \\
& agiert? \\
(o) Sprechstunden, zwischenmenschliche Kommunikation
\end{tabular}

Planung (digitale Semester)

Mediendidaktik:

Mit welchen didaktischen Lehr-/Lernformaten sind sie ad hoc in das digitale Sommersemester gegangen?

Wie sind Sie didaktisch an die Planung des darauffolgenden Semesters herangegangen? Welche Lehr-/Lernformate haben Sie genutzt?

Inwiefern gab es Angebote zur Unterstützung bei der Überführung Ihrer Veranstaltungen in digitale Formate?

Welche Programme und Plattformen haben Sie für Ihre Veranstaltung im SoSe 2020 verwendet? Welche haben Sie beibehalten im WiSe? Was kam dazu?

Technisches \& Medienkompetenz:

Wie wurden Sie in der technischen Planung der digitalen Semester unterstützt? (Rechenzentrum, Schulungen, Beratung)

Kommunikation \& Interaktion:

Wie haben Sie die Kommunikation und die Zusammenarbeit (auch zwischen den Studierenden) innerhalb und außerhalb der Veranstaltungen digital geplant?

(o) Informationen/Sprechstunden

Umsetzung

(digitale Semester)

\section{Mediendidaktik:}

Welche Erfahrungen haben Sie mit den geplanten Lehr-/Lernformaten gemacht?

(o) Was ist besonders gut gelungen? Was hat sich als schwierig herausgestellt?

(o) Was wurde verworfen? Was haben Sie neu ausprobiert?

Wie haben Sie Prüfungen abgenommen? Inwiefern hat dies so wie geplant funktioniert? 
Technisches \& Medienkompetenz:

Was hat technisch gut geklappt?

Mit welchen technischen Herausforderungen wurden Sie im Rahmen Ihrer Veranstaltungen konfrontiert?

(o) Rahmenbedingungen (z.B. Datenschutz ...)

Kommunikation \& Interaktion:

Welche Erfahrungen haben Sie mit der geplanten Kommunikation und Zusammenarbeit tatsächlich gemacht?

(o) Verhalten und Feedback von Studierenden verhalten?

\section{Chancen/ \\ Herausforderungen}

\section{Mediendidaktik:}

Welche Herausforderungen und Chancen sehen Sie durch die Digitalisierung der Lehre bzgl. der Lehr-/Lernformate?

Inwiefern hat sich die Lehre durch Corona verändert?

Welche Erfahrungen aus den digitalen Semestern können zukünftig zur Qualitätsverbesserung der Lehre beitragen?

Technisches \& Medienkompetenz:

Welche Anforderungen für die Zukunft haben Sie in Bezug auf die digitalen Werkzeuge?

Kommunikation \& Interaktion:

Welche Herausforderungen und Chancen sehen Sie durch die Digitalisierung der Lehre in Hinsicht auf die Kommunikation und Zusammenarbeit?

\begin{tabular}{ll}
\hline Abschlussfrage & Wie sieht für Sie die Lehre der Zukunft (2025) hinsichtlich der Digitali- \\
sierung aus? & Haben wir im Laufe des Interviews Aspekte ausgelassen, die Sie sich \\
vielleicht schon im Vorfeld überlegt haben oder die Ihnen gerade noch \\
durch den Kopf gehen?
\end{tabular}

Anmerkungen $(o)=$ optionale Fragen. 


\section{Literaturverzeichnis}

Adorno, T. W. (1976). Prismen: Kulturkritik und Gesellschaft. Suhrkamp.

Alexander, B., Ashford-Rowe, K., Barajas-Murphy, N., Dobbin, G., Knott, J., McCormack, M., Pomerantz, J., Seilhamer, R. \& Weber, N. (2019). Horizon Report 2019 higher education edition. EDU19.

Apelojg, B., Kiy, A., Lucke, U. \& Moebert, T. (2020). Emotionen. Stolpersteine, Krücken oder Antriebsmotor in der Hochschullehre. In M. Deimann \& T. v. Treek (Hrsg.), Digitalisierung der Hochschullehre (S. 57-71). DUZ Verlags- und Medienhaus.

Autor:innengruppe AEDiL (2021). Corona-Semester reflektiert: Einblicke einer kollaborativen Autoethnographie. wbv Media.

Bäder, J. (2020). Medienkompetenzentwicklung durch den Einsatz digitaler Medien in der Hochschullehre. In B. Fischer \& A. Paul (Hrsg.), Lehren und Lernen mit und in digitalen Medien im Sport. Springer. https://doi.org/10.1007/978-3-6 58-25524-4

Bargel, T. (2013). Studieren in Teilzeit als Beitrag zur Flexibilisierung des Hochschulstudiums: Definitionen, Daten, Konzepte, Erfahrungen, Positionen und Prognosen für Baden-Württemberg (Nr. 69). Konstanz. Arbeitsgruppe Hochschulforschung, Universität Konstanz. http://nbn-resolving.de/urn:nbn:de:bsz:352-0-26 2125

Becker, K. \& Lörz, M. (2020). Studieren während der Corona-Pandemie: Die finanzielle Situation von Studierenden und mögliche Auswirkungen auf das Studium (DZHW Brief 09/2020). Hannover. https://doi.org/10.34878/2020.09.dzhw_brief

Biggs, J. B. \& Tang, C. (2011). Teaching for quality learning at university: What the student does (4. Aufl.). SRHE and Open University Press imprint. Society for Research into Higher Education; Open University Press. https://cetl.ppu.edu/sit es/default/files/publications/-John_Biggs_and_Catherine_Tang-_Teaching_for _Quali-BookFiorg-.pdf

Bolten, R. \& Rott, K. J. (2018). Medienpädagogische Kompetenz. Anforderungen an Lehrende in der Erwachsenenbildung. Perspektiven der Praxis. MedienPädagogik (30), 137-153. https://doi.org/10.21240/MPAED/30/2018.03.05.X

Boros, N., Kiefel, K. \& Schneijderberg, K. (2020a). Kurzbefragung der Lehrenden 2020: Gesamtbericht. Freiburg. Universität Freiburg. https://www.qmlehre.uni-f reiburg.de/content-1/pdf-dokumente/berichte-befragungen/leh2020-gesamtbe richt 
Boros, N., Kiefel, K. \& Schneijderberg, K. (2020b). Kurzbefragung der Studierenden 2020: Gesamtbericht. Freiburg. Universität Freiburg. https://www.qmlehre.uni-fr eiburg.de/content-1/pdf-dokumente/berichte-befragungen/stu2020/stu2020-ge samtbericht

Bosse, E. (2021). Fachbereiche und Fakultäten in der Corona-Pandemie: Erfahrungen und Erwartungen an die Zukunft (Nr. 57). Berlin. https://hochschulforumdi gitalisierung.de/sites/default/files/dateien/HFD_AP_57_Fachbereiche_digitale _Lehre_Corona.pdf

Bremer, C. (2007). Kommunikation im E-Learning. FernUniversität in Hagen. https://vu.fernuni-hagen.de/lvuweb/lvu/file/FeU/KSW/2018SS/33052/oeff entlich/33052-7-01-S1+Vorschau.pdf

Bremer, C. (2017). Einsatz digitaler Medien in der Hochschullehre: Szenarien und Mehrwert für die Kompetenzentwicklung. In J. Erpenbeck \& W. Sauter (Hrsg.), Handbuch Kompetenzentwicklung im Netz: Bausteine einer neuen Lernwelt (S. 307-335).

Busse, B. (2017). E-Learning an Hochschulen: Stand der Entwicklung unter didaktischer Perspektive (Hefte zur Bildungs- und Hochschulforschung Nr. 90). Konstanz. https://kops.uni-konstanz.de/bitstream/handle/123456789/47466/Busse_ 2-45gyiapiz9r2.pdf?sequence $=3$ \&isAllowed $=y$

Busse, H. \& Zeeb, H. (2020). International COVID-19 Student Well-being Survey (C19 ISWS): Kurzbericht zu Ergebnissen der Online-Befragung für den Standort Bremen. https://www.uni-bremen.de/fileadmin/user_upload/fachbereiche/fb11 /Studienzentrum/Digitale_Lehre/Kurzbericht_COVID-19_ISWS_Bremen_v3_ 20200701.pdf

Cendon, E. (2018). Lifelong Learning at Universities. Future Perspectives for Teaching and Learning. Journal of New Approaches in Educational Research, $7(2), 81-87$.

CHE Centrum für Hochschulentwicklung (2020). CHECK: Digitalisierung an deutschen Hochschulen im Sommersemester 2020. https://www.che.de/download/dig italisierung-hochschulen-2020/?ind=1594986398076\&filename=CHECK_Digi talisierung_an_deutschen_Hochschulen_im_Sommersemester_2020.pdf\&wpd mdl=15118\&refresh $=609168136 f 77 f 1620142099$

Cohen, M. D. \& March, J. G. (1974). Leadership and ambiguity: The American college president. McGraw-Hill.

Cohen, M. D., March, J. G. \& Olsen, J. P. (1972). A garbage can model of organizational choice. Administrative science quarterly, 17(1), 1-25.

Creß, U. (2006). Lernorientierung, Lernstile, Lerntypen und kognitive Stile. In H. Mandl \& H. Freidricht (Hrsg.), Handbuch Lernstrategien (S. 365-377). Hogrefe.

DiMaggio, P. (1988). Interest and Agency in Institutional Theory. In L. Zucker \& G. Lynn (Hrsg.), Institutional Patterns and Organizations (S. 3-21). Ballinger. Cambridge MA 
Dresing, T. \& Pehl, T. (2018). Praxisbuch Interview, Transkription \& Analyse: Anleitungen und Regelsysteme für qualitativ Forschende. (8. Auflage). Eigenverlag.

Erkens, S. (2005). E-Learning bei der VICTORIA. In A. Hohenstein \& K. Wilbers (Hrsg.), Handbuch E-Learning (S. 1-19).

Erpenbeck, J. \& Sauter, W. (2019). Informations-, Wissens-, Kompetenz- und Wertegesellschaft. In J. Erpenbeck \& W. Sauter (Hrsg.), Stoppt die Kompetenzkatastrophe! (S. 279-300).

Feucht, T., Pistel, K.-H., Reif, C. \& Arnold, H. (2020). Die komplexen Auswirkungen des „Corona-Semesters" auf die Lehre: Die Ergebnisse der Umfrage des Studierendenrates der Universität Heidelberg. Heidelberg Inspirations for Innovative Teaching(1), 105-119. https://doi.org/10.11588/hint.2020.1.77694

Fisseler, B. (2021). Barrierefreies E-Learning an Hochschulen [Dissertation]. Technische Universität Dortmund, Dortmund. https://eldorado.tu-dortmund.de/bit stream/2003/30107/1/Dissertation.pdf

Gabriel, S. \& Pecher, H. (2020). Chancen, Herausforderungen und Entwicklungsaufgaben für E-Learning an Hochschulen: Eine qualitative Studie aus Studierendenperspektive. Open Online Journal for Research and Education(21), 1-14. https://journal.ph-noe.ac.at/index.php/resource/article/view/824/913

Gabriels, W. \& Benke-Åberg, R. (2020). Student Exchanges in Times of Crisis: Research report on the impact of COVID-19 on student exchanges in Europe. Erasmus Student Network AISBL.

Gilch, H., Beise, A. S., Krempkow, R., Müller, M., Stratmann, F. \& Wannemacher, K (2019). Digitalisierung der Hochschulen: Ergebnisse einer Schwerpunktstudie für die Expertenkommission Forschung und Innovation. Studien zum deutschen Innovationssystem. http://hdl.handle.net/10419/194284

Götzelt, K.-U. (2010). Customer Focused E-Learning: Einsatz, Gestaltung und Anwendungssysteme (Auf. 16). BoD-Books on Demand.

Handke, J. (2020). Handbuch Hochschullehre Digital: Leitfaden für eine moderne und mediengerechte Lehre (3. Aufl.). Tectum. https://doi.org/10.5771/978382887 5302

Handke, J. \& Schäfer, A. M. (2012). E-Learning, E-Teaching und E-Assessment in der Hochschullehre. Oldenbourg Wissenschaftsverlag. https://doi.org/10.1524/9 783486716849

Handke, J. \& Sperl, A. (2012). Das Inverted Classroom Model: Begleitband zur ersten deutschen ICM-Konferenz. Oldenbourg Wissenschaftsverlag. https://doi.o $\mathrm{rg} / 10.1515 / 9783486716641$

Hauschildt, J. (1997). Innovationsmanagement (2. Aufl.). Vahlens Handbücher der Wirtschafts- und Sozialwissenschaften. Vahlen.

Hodges, C., Moore, S., Lockee, B., Trust, T. \& Bond, M. (2020). The difference between emergency remote teaching and online learning. https://er.educause.edu/ articles/2020/3/the-difference-between-emergency-remote-teaching-and-onlin e-learning 
Høeg, P. (1994). Fräulein Smillas Gespür für Schnee: Roman (Ungekürzte Buchgemeinschafts-Lizenzausg). Bertelsmann.

Iberer, U. \& Müller, U. (2002). Sozialformen für E-Learning. http://docplayer.org/7 118496-Sozialformen-fuer-e-learning.html

Kercher, J. \& Plasa, T. (2020). Corona und die Folgen für die internationale Studierendenmobilität in Deutschland: Ergebnisse einer DAAD-Befragung von International Offices und Akademischen Auslandsämtern. (DAAD-Arbeitspapier). https://static.daad.de/media/daad_de/pdfs_nicht_barrierefrei/der-daad/analyse n-studien/daad_2020_corona_und_die_folgen_fuer_die_internationale_studie rendenmobilitaet_in_deutschland.pdf

Kerres, M. (2018). Mediendidaktik: Konzeption und Entwicklung digitaler Lernangebote (5. Aufl.). De Gruyter Studium. DE GRUYTER.

Kerres, M., Hanft, A., Wilkesmann, U. \& Wolff-Benedikt, K. (2012). Studium 2020.

Kindler, T., Köngeter, S. \& Schmid, T. Studieren unter Covid-19-Bedingungen: Ergebnisse der Studierendenbefragung an der FHS St.Gallen zum Thema Studieren unter Covid-19-Bedingungen. FHS St.Gallen. https://www.fhsg.ch/fileadmin/Da teiliste/5_fachhochschule/corona_2020/Studierendenbefragung-Covid-19-Bedi ngungen-Ergebnisse.pdf

Kindler, T., Köngeter, S. \& Schmid, T. (2020). Studieren unter Covid-19-Bedingungen. Ergebnisse einer Studierendenbefragung an der FHS St. Gallen zum Thema Studieren unter Covid-19-Bedingungen.

Krammer, G., Pflanzl, B. \& Matischek-Jauk, M. (2020). Aspekte der Online-Lehre und deren Zusammenhang mit positivem Erleben und Motivation bei Lehramtsstudierenden: Mixed-Method Befunde zu Beginn von COVID-19. Zeitschrift für Bildungsforschung, 10(3), 337-375. https://doi.org/10.1007/s35834-02 0-00283-2

Kreidl, C. (2011). Akzeptanz und Nutzung von E-Learning-Elementen an der Hochschule: Gründe für die Einführung und Kriterien der Anwendung von E-Learning. Waxmann. https://www.pedocs.de/volltexte/2013/8288/pdf/Kreidl_Akzeptanz_ und_Nutzung_2011_Gesamtwerk.pdf

Kreulich, K., Lichtlein, M., Zitzmann, C., Bröker, T., Schwab, R. \& Zinger, B. (2020). Hochschullehre in der Post-Corona-Zeit: Studie der bayerischen Hochschulen für angewandte Wissenschaften Sommersemester 2020. https://w3-media pool.hm.edu/mediapool/media/baukasten/img_2/fidl/dokumente_121/FIDLSt udiePostCoronaGesamt.pdf

Krueger, R. A. \& Casey, M. A. (2015). Focus groups: A practical guide for applied research ( $5^{\text {th }}$ ed.). Sage.

Kuckartz, U. (2018). Qualitative Inhaltsanalyse: Methoden, Praxis, Computerunterstützung (4. Aufl.). Grundlagentexte Methoden. Beltz Juventa.

Lermen, M. (2017). Digitalisierung und Hochschullehre. Szenarien und Mehrwert für die Kompetenzentwicklung. In J. Erpenbeck \& W. Sauter (Hrsg.), Handbuch Kompetenzentwicklung im Netz (S. 337-353). 
Lörz, M., Marczuk, A., Zimmer, L., Multrus, F. \& Buchholz, S. (2020). Studieren unter Corona-Bedingungen: Studierende bewerten das erste Digitalsemester (DZHW Brief 05/2020). Hannover. https://doi.org/10.34878/2020.05.

Luhmann, N. (1987). Grundriss einer allgemeinen Theorie. Suhrkamp.

Marczuk, A., Multrus, F. \& Lörz, M. (2021). Die Studiensituation in der CoronaPandemie: Auswirkungen der Digitalisierung auf die Lern- und Kontaktsituation von Studierenden (DZHW Brief 01/2021). Hannover. https://www.dzhw.eu/pdf /pub_brief/dzhw_brief_01_2021.pdf

Mayer, H. O. (2013). Interview und schriftliche Befragung: Grundlagen und Methoden empirischer Sozialforschung (6. Aufl.). Sozialwissenschaften 10-2012. Oldenbourg Wissenschaftsverlag. https://doi.org/10.1524/9783486717624

Mayert, A. (2017). Digitalisierung in der öffentlich geförderten Erwachsenenbildung. Große Erwartungen, wenig Unterstützung, dennoch Chancen. Forum Erwachsenenbildung(3), 14-18.

Mayring, P. \& Fenzl, T. (Hrsg.). (2014). Qualitative inhaltsanalyse. Springer VS. https://doi.org/10.1007/978-3-531-18939-0_38

Meier, R. (2006). Praxis E-Learning: Grundlagen, Didaktik, Rahmenanalyse, Medienauswahl, Qualifizierungskonzept, Betreuungskonzept, Einführungsstrategie, Erfolgssicherung (1. Aufl.). Books on Demand.

Meißelbach, C. \& Bochmann, C. (2020). „Wir können hier alle nur dazulernen“: Studierendenbefragung zur digitalen Lehre in Zeiten der Corona-Krise. Dresden. Technische Universität Dresden. https://tu-dresden.de/gsw/phil/powi/polsys/re ssourcen/dateien/forschung/umfrage-digitale-lehre/Meisselbach-Bochmann-2 020-Studierendenbefragung-Digitale-Lehre.pdf?lang=de https://doi.org/10.131 40/RG.2.2.24589.90081

Meyermann, A. \& Porzelt, M. (2014). Hinweise zur Anonymisierung von qualitativen Daten. https://www.forschungsdaten-bildung.de/files/fdb-informiert-nr-1 .pdf

Müller-Böling, D. (2005). Hochschulen weiter entfesseln - den Umbruch gestalten: Studienprogramme, Organisationsformen, Hochschultypen. Bertelsmann.

OECD (2020). Youth and COVID-19 Response, Recovery and Resilience. https://ww w.esu-online.org/?publication=youth-and-covid-19-response-recovery-and-res ilience

Oevermann, U. (1991). Genetischer Strukturalismus und das sozialwissenschaftliche Problem der Erklärung der Entstehung des Neuen. In S. Müller-Doohm (Hrsg.), Jenseits der Utopie. Theoriekritik der Gegenwart (S. 267-336). Suhrkamp.

Paepe, L. de, Zhu, C. \& Depryck, K. (2019). Development and implementation of online Dutch L2 courses in adult education. Educators' and providers' perceptions of constraints and critical success factors. Innovation in Language Learning and Teaching, 13(3), 277-291. https://doi.org/10.1080/17501229.2018.1462369

Patton, M. Q. (2002). Qualitative research and evaluation methods. Thousand Oaks. 
Pauli, P., Neuerth, S. \& Schuppert, M. (2020). Studieren in Coronazeiten. Julius-Maximilians-Universität Würzburg. https://www.uni-wuerzburg.de/aktuelles/einbl ick/single/news/studieren-in-coronazeiten/

Persike, M. \& Friedrich, J.-D. (2016). Lernen mit digitalen Medien aus Studierendenperspektive. Sonderauswertung aus dem CHE Hochschulranking für die deutschen Hochschulen. Arbeitspapier Nr. 17 des Hochschulforums Digitalisierung.

Peters, V. (2020). Ergebnisbericht zu PotsBlitz „Online-Lehre 2020“ im SoSe 2020: Bericht Studierende. Universität Potsdam. https://pep.uni-potsdam.de/media/P otsBlitz/Berichte/Studierende_PotsBlitz_Online-Lehre_2020_Allgemeiner_Ber icht.pdf

Reinmann, G. (2017). Lehren und Lernen mit Digital Natives an Hochschulen. Fünf Fragen zur Zukunft akademischen Lehrens und Lernens mit digitalen Medien. In J. Erpenbeck \& W. Sauter (Hrsg.), Handbuch Kompetenzentwicklung im Netz (S. 354-361).

Reinmann, G., Bohndick, C., Lübcke, E., Brase, A., Kaufmann, M. \& Groß, N. (2020). Emergency Remote Teaching im Sommersemester 2020: Bericht zur Begleitforschung - Lehrendenbefragung. Universität Hamburg. https://www.hul.uni -hamburg.de/dateien/begleitforschung-bericht-lehrendenbefragung-barrierefre i.pdf

Richter, T. (2017). Betriebliche Weiterbildung als Antwort auf die Implementierung von Industrie 4.0. In G. Spöttl \& L. Windelband (Hrsg.), Industrie 4.0. Risiken und Chancen für die Berufsbildung (S. 241-260). Bertelsmann.

Rogers, E. M. (1995). Diffusion of Innovations ( $4^{\text {th }}$ ed.). Free Press.

Rohs, M., Bolten, R. \& Kohl, J. (2017). Medienpädagogische Kompetenzen in Kompetenzbeschreibungen für Erwachsenenbildner* innen. Beiträge zur Erwachsenenbildung(5).

Scharmer, O. (2019). Vortrag im Rahmen der Veranstaltung Achtsam. Digital - Die Hochschule des 21. Jahrhunderts an der Universität Jena. https://achtsam.digital

Schreier, M. (2014). Varianten qualitativer Inhaltsanalyse: ein Wegweiser im Dickicht der Begrifflichkeiten. Forum Qualitative Sozialforschung, 15(1), Artikel 18.

Schulmeister, R. \& Loviscach, J. (2017). Mythen der Digitalisierung mit Blick auf Studium und Lernen. In C. Leinweber \& C. de Witt (Hrsg.), Digitale Transformation im Diskurs (S. 1-21). https://ub-deposit.fernuni-hagen.de/receive/mir_ mods_00001055

Schumacher, F., Ademmer, T., Bülter, S. \& Kneiphoff, A. (2021). Hochschulen im Lockdown: Lehren aus dem Sommersemester 2020 (Nr. 58). Berlin. https://hochs chulforumdigitalisierung.de/sites/default/files/dateien/HFD_AP_58_Hochschu len_im_Lockdown.pdf

Seyfeli, F., Elsner, L. \& Wannemacher, K. (2020). Vom Corona-Shutdown zur Blended University? ExpertInnenbefragung Digitales Sommersemester. Tectum. 
Sgier, I., Haberzeth, E. \& Schüepp, P. (2018). Digitalisierung in der Weiterbildung. Ergebnisse der jährlichen Umfrage bei Weiterbildungsanbietern. http://edudoc.ch /record/130478/files/SVEB_Weiterbildungsstudie2017_2018.pdf

Spitzer, M. (2014). Digitale Demenz: Wie wir uns und unsere Kinder um den Verstand bringen (10. Aufl.). Droemer HC.

Stammen, K.-H. \& Ebert, A. (2020). Noch online? Studierendenbefragung zur medientechnischen Ausstattung im Sommersemester 2020: Gesamtbericht. Ergebnisse der universitätsweiten UDE-Umfrage im Sommersemester 2020. Universität Duisburg-Essen. https://www.uni-due.de/imperia/md/images/zhqe/stammenebert_ 2020_nochonline_gesamtbericht.pdf

Stathopoulou, T., Mouriki, A. \& Papaliou, O. (2020). Student well-being during the covid-19 pandemic in greece: Results from the C-19 ISWS Survey. National Centre for Social Research (EKKE). https://www.researchgate.net/profile/Theo niStathopoulou/publication/344493432_STUDENT_WELL-BEING_DURING _THE_COVID-19_PANDEMIC_IN_GREECE_RESULTS_FROM_THE_C 19 _ISWS_SURVEY/links/5f7c5f4492851c14bcb17bf1/STUDENT-WELL-BEING -DURING-THE-COVID-19-PANDEMIC-IN-GREECE-RESULTS-FROM-TH E-C-19-ISWS-SURVEY.pdf

Szczyrba, B. (2006). The Shift from Teaching to Learning. Zeitschrift für Psychodrama und Soziometrie(5), 47-58.

Traus, A., Höffken, K., Thomas, S., Mangold, K. \& Schröer, W. (2020). Stu.diCo. - Studieren digital in Zeiten von Corona. Hildesheim. Universitätsverlag Hildesheim. https://doi.org/10.18442/150

Universität Bielefeld. (2020). Studierendenbefragung zum Sommersemester 2020: vorläufige Ergebnisse. https:/uni-bielefeld.de/themen/qm-studium-lehre/befrag ungen-monitoring/studierendenbefragung/sommersemester2020/

Vallaster, C. \& Sageder, M. (2020). Verändert Covid-19 die Akzeptanz virtueller Lehrformate in der Hochschulausbildung? Implikationen für die Hochschulentwicklung. Zeitschrift für Hochschulentwicklung (ZFHE), 15(4), 281-301. https:// doi.org/10.3217/ZFHE-15-04/16

van de Velde, S., Buffel, V., Wouters, E., van Hal, G., Bracke, P. \& Colman, L. (2020). COVID-19 international student well-being study: Eerste resultaten belgische cijfers. Universiteit Antwerpen.

VERBI Software (2019). MAXQDA 2020 [Computer software]. Berlin. maxqda.com

Vester, F. (2007). Denken, Lernen, Vergessen (31. Auflage). dtv.

Winde, M., Werner, S., Gumbmann, B. \& Hieronimus, S. (2020). Hochschulen, Corona und jetzt? Wie Hochschulen vom Krisenmodus zu neuen Lehrstrategien für die digitale Welt gelangen. Berlin. Stifterverband für die Deutsche Wissenschaft e.V. https://www.future-skills.net/analysen/hochschulen-corona-und-jetzt 
